\title{
Cascaded Lyapunov Vector Fields for Spacecraft Relative Trajectory Tracking in Rotating Reference Frames Under Acceleration Constraints
}

\author{
by \\ Jeffrey G.W. Hough \\ A thesis submitted to the Faculty of Graduate and Postdoctoral Affairs \\ in partial fulfillment of the requirements for the degree of \\ Master of Applied Science \\ in \\ Mechanical Engineering \\ Carleton University \\ Ottawa, Ontario \\ (C) 2021 \\ Jeffrey G.W. Hough
}


This work is dedicated to my parents, Allan and Joanne Hough.

Thank you for your endless support in every path that I take. Without the opportunities that you have worked so hard to provide me, finding my passion for research would have never been possible.

I love and admire you both. 


\section{Abstract}

Autonomous docking to an uncontrolled tumbling target is a complex maneuver, requiring satisfaction of several path constraints while respecting the acceleration limitations of the controlled vehicle. Furthermore, as is always the case in spacecraft applications, some level of fuel optimization is required. Presently, methods for achieving autonomous docking usually rely on high-dimensional or computationally complex optimizations which, to avoid the use of open-loop control, are required to run in real-time. Unfortunately, it is well-known that spacecraft flight-computers are highly limited in their computational power, rendering many of these methods impractical.

In this work, a novel approach to autonomous docking is explored within the framework of Lyapunov vector fields. To achieve this design, a substantial extension to Lyapunov vector fields (herein referred to as a cascaded Lyapunov vector field) is first presented which broadens applications to cases where the desired final trajectory is defined in a tumbling and accelerating reference frame. Using this novel extension, a two-phase docking maneuver is developed: in Phase 1, the chaser spacecraft converges from initially large distances, and gradually tracks the target attitude dynamics until it has aligned with the docking port at a desired distance. Then, in Phase 2, the chaser contracts into the final docking position. Using the methods developed in this thesis, the path constraints are satisfied by construction of the various vector fields, and the acceleration constraint is satisfied by bounding parameters of the guidance in each phase. Moreover, a performance optimization technique is developed based on estimations of fuel usage and maneuver time which are only a function of these bounded parameters. Importantly, this new method requires no in-the-loop optimizations, and therefore retains feasibility for real-time implementation on board spacecraft. The performance of this novel docking technique is then confirmed in simulation. Planar experimental validation confirms that the method is robust to real-world disturbances such as measurement noise, imperfect thruster resolution, time-delays, and unmodeled perturbations. 


\section{Acknowledgements}

Firstly, my deepest gratitude goes to my supervisor, Steve Ulrich. Thank you for your never ending guidance and patience. Thank you for always allowing me the freedom to explore every avenue of my research, even the questionable ones. Thank you for working so tirelessly to ensure that I, and all of your students, receive every available opportunity to advance our knowledge and education. Thank you for making me realize that I tend to write repetitively (and for reducing my use of parentheses). I will be forever grateful that I had you as my mentor.

I have had countless friends and colleagues who have, in some way, helped me directly with this work. I would like to particularly thank Alex Crain and Kirk Hovell for the hours of help with lab equipment and software. As both of you have discovered, I am magnificently efficient at breaking things. I hope your tech-support skills are never tested so rigorously again. The laboratory component of this research would not have been possible without you both. Further, I would like to thank Yazan Chihabi, Raha Hojjati, Samuel Lortie, Abin Pothen, and so many others who either helped with experiments in the lab, or at some point talked me through concepts contained in this work. I would like to especially thank Andriy Predmyrskyy, who spent countless hours as my pandemic lab-buddy. I am sorry I always made you wake up so early. I am grateful that I've had you to bounce ideas with, even if many of your ideas go way over my head. I am also thankful to have become closer friends with you in these strange times.

To Alyssa; you have been my constant source of support, of confidence, and of motivation. You have also been my reminder to live a life with balance. I have been so lucky to have you by my side throughout this chapter of my life, and I cannot wait to start the next chapter of my life with you.

To my family, and my friends outside of the lab; thank you for always supporting me in your own ways. I am so fortunate to receive the encouragement and love that I do from all of you. 
This research was financially supported in part by the Natural Sciences and Engineering Research Council of Canada, through an Alexander Graham Bell Canada Graduate Scholarship. Furthermore, I am very thankful to have received support from Carleton University through a variety of scholarships and teaching assistant positions. 


\section{Table of Contents}

Abstract $\quad$ iii

Acknowledgements $\quad$ iv

List of Tables $\quad$ X

List of Figures $\quad$ xii

Chapter 1 Introduction 1

1.1 Motivation . . . . . . . . . . . . . . . . . 1

1.2 Problem Statement . . . . . . . . . . . . . . . . 5

1.3 Previous Work . . . . . . . . . . . . . . . 7

1.4 Thesis Objectives . . . . . . . . . . . . . . . . 9 9

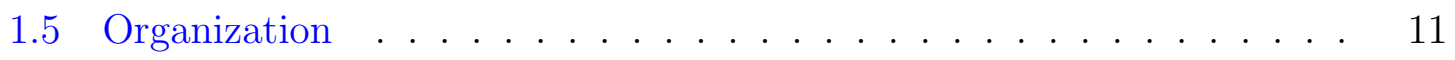

Chapter 2 Review of Lyapunov Vector Fields Theory 12

2.1 General Lyapunov Vector Field Theory . . . . . . . . . . . . . . . . . 12

2.2 Lyapunov Vector Fields for Unmanned Air Vehicles . . . . . . . . . . 15

Chapter 3 Development of Cascaded Lyapunov Vector Fields 18

3.1 Problem Formuation . . . . . . . . . . . . . . . . . . . 18

3.1.1 Reference Frame and Vector Definitions . . . . . . . . . . . . . 19

3.1.2 Tracking Lyapunov Vector Fields in Rotating Reference Frames 21

3.2 General Cascaded Lyapunov Vector Fields: Structure and Stability . 22

3.2.1 Basic Structure of the Cascaded Lyapunov Vector Field . . . . 22

3.2.2 Stability . . . . . . . . . . . . . . 26

3.2.3 Simplifying Assumptions to Satisfy Stability Conditions . . . . 29

3.2.4 Robustness of the Alignment Field Stability to Small Contraction Errors . . . . . . . . . . . . . . . . . . . . . 30 
3.3 Acceleration Constraints . . . . . . . . . . . . . . . . . . . 33

3.3 .1 Simplified Form of CLVF . . . . . . . . . . . . . 34

3.3.2 Upper-Boundedness of the Required Tracking Acceleration . . 37

3.3.3 Feed-Forward Acceleration Equations and Interpretation . . . 41

3.3.4 Tracking the CLVF with Saturation . . . . . . . . . . . . 43

3.4 Design Procedure and Simulations . . . . . . . . . . . . . 46

3.4.1 Scenario Description . . . . . . . . . . . . . . 46

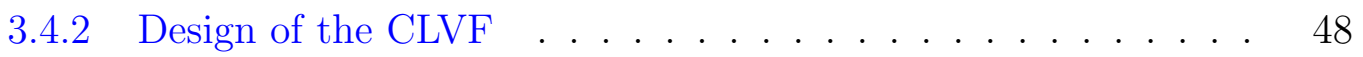

3.4 .3 Results . . . . . . . . . . . . . . . . . . 52

3.5 Conclusions . . . . . . . . . . . . . . . . . . . 62

$\begin{array}{lll}\text { Chapter } 4 & \text { Docking Problem Formulation }\end{array}$

4.1 Reference Frame Definitions . . . . . . . . . . . . . . . . . . . 64

4.1.1 Earth-Centered Inertial (ECI) Reference Frame . . . . . . . . 64

4.1.2 Body-Fixed Reference Frame . . . . . . . . . . . 65

4.2 Spacecraft Dynamics . . . . . . . . . . . . . . . 65

$4.2 .1 \quad$ ECI Orbital Dynamics . . . . . . . . . . . . . . . . 66

4.2.2 Body-Fixed Attitude Dynamics _ . . . . . . . . . . 67

4.3 Docking Phases . . . . . . . . . . . . . . . . . . . . . . . . 68

4.4 Target and Chaser Spacecraft Vector Definitions . . . . . . . . . 68

4.5 Feedback Structure . . . . . . . . . . . . . . . . . . . 71

$\begin{array}{lll}\text { Chapter } 5 & \text { Docking Maneuver Design } & \mathbf{7 4}\end{array}$

5.1 Phase 0 Design . . . . . . . . . . . . . . . . . . . . . . 74

5.2 Phase 1 Design . . . . . . . . . . . . . . . . . 75

5.2 .1 Guidance Law . . . . . . . . . . . . . . . . . . 75

5.2.2 Upper Bound of Required Tracking Acceleration . . . . . . . . 77

5.3 Phase 2 Design . . . . . . . . . . . . . . . . . . . . . . 80

5.3 .1 Guidance Law . . . . . . . . . . . . . . . . . . . . 80

5.3.2 Upper Bound of Required Tracking Acceleration . . . . . . . . 82

5.4 Parameter Optimization Design . . . . . . . . . . . . . . 87 
5.4.1 Time and Fuel Approximation Functions . . . . . . . . . . . . 89

5.4.2 Optimization Cost Function . . . . . . . . . . . . 96

5.4.3 Interior Point Optimizer with Newton Step Design .... 99

5.5 Switching Conditions . . . . . . . . . . . . . . 101

$\begin{array}{lll}\text { Chapter } 6 & \text { Simulations } & 102\end{array}$

6.1 Simulation Scenario . . . . . . . . . . . . . . . . . . 102

6.2 Results......................... 104

6.2.1 Acceleration Constraint and Path Constraint Results . . . . . 104

6.2.2 Parameter Optimization Results . . . . . . . . . . . . 106

$\begin{array}{lll}\text { Chapter } 7 & \text { Experimental Validation } & 143\end{array}$

7.1 Spacecraft Proximity Operations Testbed (SPOT) Facility . . . . . . 143

7.2 Experiment Scenario Definition . . . . . . . . . . . . . . . 145

7.2.1 Docking Geometry . . . . . . . . . . . . . . 146

7.2.2 Parameter Optimization . . . . . . . . . . . . . 146

7.2.3 Switching Conditions . . . . . . . . . . . . . 146

7.2.4 Control Parameter . . . . . . . . . . . . . . . 146

7.2.5 Initial Conditions . . . . . . . . . . . . . . . . . 147

7.3 Results . . . . . . . . . . . . . . . . . . . 147

$\begin{array}{lll}\text { Chapter } 8 & \text { Conclusions } & 157\end{array}$

8.1 Thesis Summary . . . . . . . . . . . . . . . . . 157

8.2 Improvements and Future Work . . . . . . . . . . . . . . . . 158

8.3 Significance of Work . . . . . . . . . . . . . . . . . . . . 159

Appendix A Evaluation of the CLVF robustness $\quad 160$

Appendix B Conversion from Orbital Elements to Initial ECI Position and Velocity 162

Appendix C Gradient and Hessian of Cost Functions 164 
References 


\section{List of Tables}

3.1 Parameter selection for spacecraft with varying acceleration constraints. . . . . . . . . . . . . . . . 51

3.2 Alignment robustness to contraction errors for each $u_{\max }$. . . . 52

3.3 Initial conditions. . . . . . . . . . . . . . 53

6.1 Target dynamic and geometric parameters. . . . . . . . . . 102

6.2 Target orbit parameters. . . . . . . . . . . . . . . 102

$6.3 \quad$ Simulation initial conditions. . . . . . . . . . . . 103

6.4 Simulation weights. . . . . . . . . . . . . . 104

6.5 Actual $\Delta \nu_{P 1}(\mathrm{~m} / \mathrm{s})$ for weight case $1 \ldots \ldots \ldots$. . . . . 138

6.6 Actual $\Delta \nu_{P 1}(\mathrm{~m} / \mathrm{s})$ for weight case $2 \ldots \ldots \ldots$. . . . . 138

$6.7 \quad$ Actual $\Delta \nu_{P 1}(\mathrm{~m} / \mathrm{s})$ for weight case $3 \ldots \ldots \ldots$. . . . . . 138

$6.8 \quad$ Actual $\Delta \nu_{P 1}(\mathrm{~m} / \mathrm{s})$ for weight case $4 \ldots \ldots \ldots$. . . . . 138

$6.9 \quad$ Actual $\Delta \nu_{P 1}(\mathrm{~m} / \mathrm{s})$ for weight case $5 \ldots \ldots \ldots$. . . . . 139

6.10 Actual $T_{P 1}(\mathrm{~s})$ for weight case 1. . . . . . . . . . . . 139

6.11 Actual $T_{P 1}(\mathrm{~s})$ for weight case 2. . . . . . . . . . . . . 139

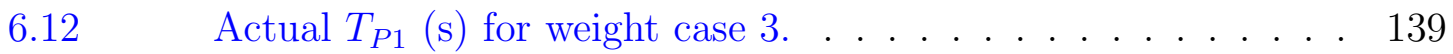

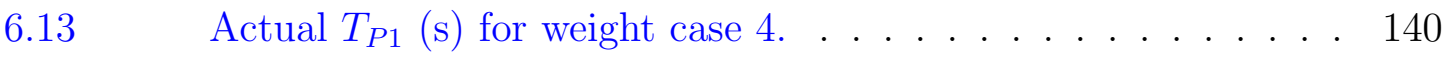

6.14 Actual $T_{P 1}(\mathrm{~s})$ for weight case 5. . . . . . . . . . . . 140

6.15 Actual $\Delta \nu_{P 2}(\mathrm{~m} / \mathrm{s})$ for weight case $1 \ldots \ldots \ldots$. . . . . . 140

6.16 Actual $\Delta \nu_{P 2}(\mathrm{~m} / \mathrm{s})$ for weight case $2 \ldots \ldots \ldots$. . . . . 140

$6.17 \quad$ Actual $\Delta \nu_{P 2}(\mathrm{~m} / \mathrm{s})$ for weight case $3 \ldots \ldots \ldots \ldots$. . . . 141

6.18 Actual $\Delta \nu_{P 2}(\mathrm{~m} / \mathrm{s})$ for weight case $4 \ldots \ldots \ldots$. . . . . 141

$6.19 \quad$ Actual $\Delta \nu_{P 2}(\mathrm{~m} / \mathrm{s})$ for weight case 5. . . . . . . . . . . . 141

$6.20 \quad$ Actual $T_{P 2}(\mathrm{~s})$ for weight case 1. . . . . . . . . . . 141

$6.21 \quad$ Actual $T_{P 2}(\mathrm{~s})$ for weight case 2. . . . . . . . . . . 142

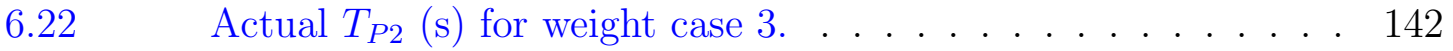

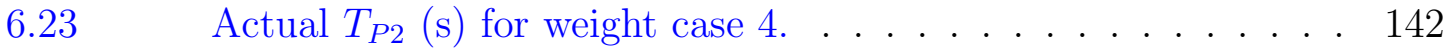


6.24 Actual $T_{P 2}(\mathrm{~s})$ for weight case 5. . . . . . . . . . . . . . 142

7.1 RED docking geometric parameters. . . . . . . . . . 146

7.2 Experiment initial conditions. . . . . . . . . . . . . 147 


\section{List of Figures}

$2.1 \quad$ Lyapunov vector fields with $\eta=3, \hat{\boldsymbol{n}}$ in vertical direction. . . . 17

$3.1 \quad$ Reference frame and vector definitions. . . . . . . . . . . 19

$3.2 \quad V_{c}$ contours in two different orientations. . . . . . . . . . . 23

3.3 Desired velocity remaining approximately constant from modest initial position separations. . . . . . . . . . . . . . 45

3.4 Desired inspection trajectory. . . . . . . . . . . . 47

3.5 Maximum acceleration constraint: $u_{\max }=5.0 \mathrm{~m} / \mathrm{s}^{2} \ldots \ldots$. . 54

$3.6 \quad$ Maximum acceleration constraint: $u_{\max }=3.0 \mathrm{~m} / \mathrm{s}^{2} \ldots \ldots 5$

3.7 Maximum acceleration constraint: $u_{\max }=1.0 \mathrm{~m} / \mathrm{s}^{2} \ldots \ldots$

3.8 Maximum acceleration constraint: $u_{\max }=0.80 \mathrm{~m} / \mathrm{s}^{2} \ldots \ldots$. . . 57

$3.9 \quad$ Maximum acceleration constraint: $u_{\max }=0.70 \mathrm{~m} / \mathrm{s}^{2} \ldots \ldots$

3.10 Time-lapse of $u_{\max }=5.0 \mathrm{~m} / \mathrm{s}^{2}$ - case 1. . . . . . . . . 59

3.11 Time-lapse of $u_{\max }=0.7 \mathrm{~m} / \mathrm{s}^{2}-$ case $1 \ldots \ldots$. . . . . 60

$4.1 \quad$ Definition of Earth-centered inertial reference frame. . . . . . . 65

$4.2 \quad$ Docking phases. . . . . . . . . . . . . . . . 69

4.3 Target and chaser vector definitions. . . . . . . . . . 70

$4.4 \quad$ Alignment geometry for phases one and two. . . . . . . . . . 71

4.5 High level feedback structure. . . . . . . . . . . . . . . 72

4.6 Feedback structure of LVF and CLVF systems. . . . . . . . 73

$5.1 \quad$ Contraction velocity function, $k_{c}=1, b=4 \ldots \ldots 76$

$5.2 \quad$ Illustration of Phase 2 LVF guidance under nominal conditions. 83

5.3 Illustration of Phase 2 LVF guidance initialized with significant tracking error. . . . . . . . . . . . . . . 84

$5.4 \quad$ Chaser arriving on attractor with initial misalignment. . . . . 90

5.5 Two $\Delta \nu$ maneuvers to contract to the radius $\alpha \ldots \ldots 3$

$6.1 \quad$ Acceleration constraint $u_{\max }=1 \mathrm{~m} / \mathrm{s}^{2}$, simulation case $1 \ldots \ldots 107$ 
$6.2 \quad$ Acceleration constraint $u_{\max }=2 \mathrm{~m} / \mathrm{s}^{2}$, simulation case $1 . \ldots 108$

$6.3 \quad$ Acceleration constraint $u_{\max }=3 \mathrm{~m} / \mathrm{s}^{2}$, simulation case $1 . \ldots 109$

$6.4 \quad$ Acceleration constraint $u_{\max }=4 \mathrm{~m} / \mathrm{s}^{2}$, simulation case 1 . . . 110

$6.5 \quad$ Acceleration constraint $u_{\max }=5 \mathrm{~m} / \mathrm{s}^{2}$, simulation case 1 . . . 111

6.6 Acceleration constraint $u_{\max }=1 \mathrm{~m} / \mathrm{s}^{2}$, simulation case 4 . . . 112

$6.7 \quad$ Acceleration constraint $u_{\max }=2 \mathrm{~m} / \mathrm{s}^{2}$, simulation case 4 . . . 113

$6.8 \quad$ Acceleration constraint $u_{\max }=3 \mathrm{~m} / \mathrm{s}^{2}$, simulation case 4 . . . 114

$6.9 \quad$ Acceleration constraint $u_{\max }=4 \mathrm{~m} / \mathrm{s}^{2}$, simulation case 4 . . . 115

$6.10 \quad$ Acceleration constraint $u_{\max }=5 \mathrm{~m} / \mathrm{s}^{2}$, simulation case 4 . . 116

$6.11 \quad$ Acceleration constraint $u_{\max }=1 \mathrm{~m} / \mathrm{s}^{2}$, simulation case 7 . . . 117

6.12 Acceleration constraint $u_{\max }=2 \mathrm{~m} / \mathrm{s}^{2}$, simulation case 7. . . 118

$6.13 \quad$ Acceleration constraint $u_{\max }=3 \mathrm{~m} / \mathrm{s}^{2}$, simulation case $7 \ldots$. . 119

$6.14 \quad$ Acceleration constraint $u_{\max }=4 \mathrm{~m} / \mathrm{s}^{2}$, simulation case 7 . . . 120

$6.15 \quad$ Acceleration constraint $u_{\max }=5 \mathrm{~m} / \mathrm{s}^{2}$, simulation case 7. . . 121

6.16 Time-lapse of three-dimensional docking simulation. . . . . . . 125

6.17 Initial condition set $1, u_{\max }=5.0 \mathrm{~m} / \mathrm{s}^{2}$, varying weigh sets. . . 127

6.18 Initial condition set $2, u_{\max }=3.0 \mathrm{~m} / \mathrm{s}^{2}$, varying weigh sets. . 128

6.19 Actual versus estimated $\Delta \nu_{P 1}$ for initial condition set $1, u_{\max }=$ $5.0 \mathrm{~m} / \mathrm{s}^{2}$, across all weight sets. . . . . . . . . . . 130

6.20 Actual versus estimated $\Delta \nu_{P 1}$ for initial condition set 1 and 2 , $u_{\max }=\{1.0,2.0,3.0,4.0,5.0\} \mathrm{m} / \mathrm{s}^{2}$, across all weight sets. . . . 131

6.21 Actual versus estimated $\Delta \nu_{P 1}$ for initial condition set $6, u_{\max }=$ $\{1.0,2.0,3.0,4.0,5.0\} \mathrm{m} / \mathrm{s}^{2}$, across all weight sets. . . . . . 131

6.22 Actual versus estimated $T_{P 1}$ for initial condition set $6, u_{\max }=$ $\{1.0,2.0,3.0,4.0,5.0\} \mathrm{m} / \mathrm{s}^{2}$, across all weight sets. . . . . . 132

6.23 Actual versus estimated fuel usage for Phase 1 over 175 cases. 133

6.24 Actual versus estimated maneuver time for Phase 1 over 175 cases. . . . . . . . . . . . . . . . . . 134

6.25 Actual versus estimated fuel usage for Phase 1, omitting weight set 5............................. 135 
6.26 Actual versus estimated fuel usage for Phase 1 over 175 cases. 136

6.27 Actual versus estimated maneuver time for Phase 1 over 175 cases. . . . . . . . . . . . . . . . . . . . 137

7.1 Top view of granite table and robotic platforms in SPOT facility.144

7.2 Definition of reference frames and attitude measurements. . . 145

7.3 Experimental results, initial condition case 1. . . . . . . . 149

$7.4 \quad$ Experimental results, initial condition case $2 \ldots \ldots \ldots \ldots$

7.5 Experimental results, initial condition case 3. . . . . . . . 151

7.6 Experimental results, initial condition case 4. . . . . . . . 152

7.7 Experimental results, initial condition case 5. . . . . . . 153

$7.8 \quad$ Initial condition set 1 , timelapse. . . . . . . . . . 154

7.9 Initial condition set 3 , timelapse. . . . . . . . . . 155

$7.10 \quad$ Initial condition set 5 , timelapse. . . . . . . . . 156 


\section{Chapter 1}

\section{Introduction}

\subsection{Motivation}

Under a critical density of debris orbiting Earth, it is possible for a single collision to cascade into an exponentially-increasing chain of collisions. First theorized by Donald J. Kessler in 1978 [1], the Kessler Syndrome has the potential to cause a debris-belt, closing off certain orbits or impeding mankind from launching into space entirely. Unfortunately, every year the orbital space surrounding Earth becomes increasingly crowded with satellites, about $37 \%$ of which are completely dysfunctional ${ }^{1}$. Recent years in particular have seen a tremendous increase in the number of satellite launches. For example, between the launch of Sputnik 1 in 1957 and the year 2012, the largest number of satellites launched in one year occurred in 1990 (168 satellites launched according to the United Nations Office for Outer Space Affairs ${ }^{2}$ ). Since 2013, consistently above 200 satellites have been launched every year. In 2017 and 2018, a total of 456 and 453 launches occurred respectively. In 2019, a record-breaking 583 launches. In the year 2020 alone, 1264 launches occurred. In just the first three months of 2021, over 500 satellites have already been launched. In contrast, only 410 satellites returned to Earth in $2020^{3}$. In the same three month time period of 2021, only 100 satellites have deorbited.

There are, of course, plans to significantly increase the number of satellites in Low Earth Orbit (LEO) for global internet services such as Starlink by SpaceX ${ }^{4}$. While global internet coverage certainly has tremendous benefits for humanity, several governments worldwide have become concerned with the increasing population of space

\footnotetext{
${ }^{1}$ ESA, https://www.esa.int/Safety_Security/Space_Debris/Space_debris_by_the_numbers

${ }^{2}$ UNOOSA, http://www.unoosa.org/oosa/osoindex/search-ng.jspx?lf_id=

${ }^{3}$ Space-Track, https://www.space-track.org/decay

${ }^{4}$ SpaceX, https://www.starlink.com/
} 
debris. For example, recent programs such as NASA's Restore L ${ }^{5}$, DARPA's Robotic Servicing of Geosynchronous Satellites ${ }^{6}$, and the ESA's Clean Space Initiative ${ }^{7}$ are all under the common motivation of eliminating the space debris problem. The Canadian Department of National Defence has also recently been providing increased funding for research related to debris removal, such as through the Collision Course project as part of the IDEaS program ${ }^{8}$. In particular, research into autonomous robotic spacecraft has been of increasing interest. Indeed, with a growing debris population, robots that can perform routine tasks autonomously (such as simple repairs/maintenance, refueling, or debris removal) may become increasingly essential for actively preventing disaster.

Beyond such grim concerns, there are also commercial benefits to the development autonomous servicing spacecraft. Consider the fact that presently, nearly all spacecraft are assembled on Earth before their launch into orbit. This sounds quite sensible; however, this traditional production method comes with limitations. For example, assembled spacecraft must fit within launch fairings at most 5 meters wide [2]. In addition, to survive the harsh vibrations of launch, spacecraft joints must be reinforced using mass-inefficient methods (such as packing materials). Such methods are costly, as launch vehicles are very limited in the mass they can carry to orbit (typically, about $5 \%$ of the launcher mass is payload). One alternate production method is to perform assembly after launching the materials. For instance, the International Space Station (ISS) would have been impossible to build on Earth due to its size; built by astronauts, the ISS took more than 40 missions that spanned over 10 years to complete. For a future with multiple large and complex space structures, having astronauts perform in-space assembly may not be scalable. To this end, a partnership (known as the Space Science and Technology Partnership) combining the U.S. Air Force, National Aeronautics and Space Institute, National Reconnaissance

\footnotetext{
${ }^{5}$ NASA Restore-L Robotic Servicing Mission. https://sspd.gsfc.nasa.gov/restore-l.html.

${ }^{6}$ DARPA Robotic Servicing of Geosynchronous Satellites, http://www.darpa.mil/program/roboticservicing-of-geosynchronous-satellites.

${ }^{7}$ ESA Clean Space, http://www.esa.int/Our_Activities/Space_Engineering_Technology/Clean_Space.

${ }^{8} \mathrm{CSA}, \quad$ https://www.canada.ca/en/department-national-defence/programs/defence-ideas/pastopportunities/space-debris-mitigation.html
} 
Office, Defense Advanced Research Projects Agency, and U.S. Naval Research Laboratory has been focused on the development of technologies for autonomous in-orbit manufacturing and assembly of spacecraft [3].

Clearly, technologies developed for a) debris removal, b) damaged-spacecraft servicing, or c) assembling a new spacecraft, will have several similarities. One such similarity is that in all cases, the controlled spacecraft will routinely perform autonomous rendezvous and docking procedures to uncooperative targets. In general, this target may be tumbling and orbiting the Earth elliptically.

Many authors have proposed methods which aim to solve the complex problem of docking to tumbling targets. One very popular and effective approach to autonomous docking from the literature is Model Predictive Control (MPC) [4-8]. Using MPC, both the guidance and control problems can be solved simultaneously, and some level of fuel-optimization is achieved. In addition, MPC allows for the consideration of several linear and nonlinear constraints such as thrust-limitations and keep-out zones. Several frameworks for MPC exist, however, they all share the same general computational approach: first, a finite time-horizon optimization is solved based on a model of the dynamics to find the lowest cost trajectory as measured by some combination of input effort and output errors. This process results in a vector of future inputs which are "optimal", at least over the finite-time horizon considered. After this optimization, only the first step of the path is implemented, and then the process repeats. By performing only the first step, MPC avoids long durations of open-loop control, which is a strategy with many well-known weaknesses, such as having no robustness to unmodeled disturbances [9]. Inherently, the main hurdle of this approach is that it is computationally-expensive to solve an optimization problem at every time-step. Unfortunately, computers on-board space bound vehicles are well-known for having abysmal processing power. For example, the most recent Mars rover, Perseverance (2021), is highlighted by NASA for having 10 times the processing speed of previous rovers, and "Better Memory Than Ever" 9 . However, the on-board processor runs at just 200 megahertz, which is still over 10 times slower than even most budget-laptops. Likewise the rover holds only 2 gigabytes of flash memory, and

\footnotetext{
${ }^{9}$ https://mars.nasa.gov/mars2020/spacecraft/rover/brains/
} 
just 256 megabytes of Dynamic Random Access Memory. Assuming MPC is running at $10 \mathrm{~Hz}$ with a time-horizon of just one second, and that all three spatial Cartesian coordinates must be optimized, this results in an optimization which must solve for a 30 by 1 vector every 0.1 seconds. This, of course, ignores that there are several acceleration, velocity, and path constraints at each timestep which complicate the optimization, and that the algorithm must simultaneously propagate a model of the target spacecraft dynamics in parallel.

Perhaps as a result of this computational-expense, proposed methods of MPC often only present simulations with planar motion $[5,7]$ and/or targets that are nonrotating/rotating with a fixed angular velocity $[5,7,8]$. Li et al. [6] designed an MPC for tumbling targets. However, there are again dynamics simplifications, as the target is assumed to be axisymmetric, allowing for simplified closed-form attitude equations [10]. These simplifications are particularly important because MPC is model-based, and therefore simplifications to the dynamics model ultimately leads to lighter computational load, benefiting the algorithm performance.

Another combined guidance and control method developed for docking maneuvers is Inverse Dynamics-Based Trajectory Planning, such as proposed by Ventura et al. [11]. Using this method, the docking problem is characterized by a high-order polynomial, and is inverted to be solved as a nonlinear programming problem using solvers such as the Sparse Nonlinear OPTimizer (SNOPT). Path constraints can be enforced by fixing particular polynomial coefficients, whereas other coefficients are optimized to find the lowest-energy trajectory in terms of fuel usage. The input is then characterized as a time-function which uses the optimized coefficients. Similar to MPC, the coefficients are re-optimized periodically. One major difference from MPC is that the trajectory coefficients are not re-optimized at every time-step. Rather, the trajectory is updated as fast as possible, and between updates, the controlled vehicle follows the previous trajectory update in open-loop. In Ref. [11], the target orbit is assumed to be circular, however, the full nonlinear attitude dynamics of both spacecraft are considered. While the method is shown to be quite effective in considering optimizations while satisfying constraints, the computational times presented for this 
method are quite significant. In fact, an optimization time of 10 seconds per trajectory update was selected as a feasible duration using a laptop with an Intel Core i7 U $2.8 \mathrm{GHz}$ processor and 8.0 GB of RAM (note that control inputs are still computed very fast; the 10 second optimization cycle implies that the guidance and control are open-loop for 10 seconds at a time). These computational times increase as the polynomial becomes more parameterized, representing a trade-off between path optimality and computational feasibility. As has already been discussed, computational feasibility issues will be exacerbated on small servicing spacecraft with significantly reduced computational resources compared to the computer specifications cited by [11].

The Artificial Potential Field approach is an elegant solution to the docking problem, such as presented by Lopez and McInnes [12]. Using this approach, regions of low and high energy can be imposed on the state-space leading to implicit path constraints using an analytical guidance and control approach. There are certain constraints which are not considered, however, such as maximum thrust capacity. Furthermore, this method is typically intended for cases where the target has a fixed attitude and position, particularly when the target has a complex but well-known shape.

\subsection{Problem Statement}

From the previous discussion, there are effectively four criterion which are essential for an autonomous docking algorithm to be useful in practice:

- path constraints must be met to successfully dock while avoiding collisions with the target;

- thrust constraints of the chaser vehicle must be respected, such that the guidance and control commands are feasible to track;

- some level of fuel optimization must be achieved, as is always required for space applications where fuel resources are limited; and

- the algorithm must be computationally-light, so as to be realistic for implementation on-board modern space flight-computers. 
All of the algorithms discussed thus far present challenges for at least one of these criterion. Therefore, this thesis will seek an alternate method of solving the docking problem.

Contrasting the powerful but computationally-heavy algorithms outlined above, one simple guidance method which has arisen for UAVs is the kinematics-based Lyapunov vector field (LVF). As is well known, the typical use for a Lyapunov function is to prove the stability of a given system [13]. Lyapunov vector fields, however, invert this process, and instead begin with a desirable Lyapunov function, and then use its properties to create guidance and control laws which are inherently asymptotically stable.

Generally speaking, LVFs are a predefined mapping from each position in space to a desired velocity. Therefore, constraints such as velocity or acceleration limitations can be addressed directly by judicious design of this mapping. Furthermore, path constraints can be indirectly addressed by shaping the LVF to avoid undesirable regions.

Citing the characteristics above, LVFs are a strong candidate for a low-computation approach to spacecraft docking. However, constructing a LVF for space applications requires very significant alterations to the existing framework. For example, all works relating to UAV applications have been subject to a constant (or nearly constant) inertial velocity constraint for remaining in steady flight. With spacecraft maneuvers, however, maintaining a constant velocity becomes unnecessary, and in fact impractical. One obvious example is that during docking, the desired relative velocity between the controlled spacecraft and uncontrolled target should converge to zero as the two spacecraft are brought into contact. Indeed, rather than being constrained by velocity, spacecraft are limited by their acceleration capabilities.

Additionally, in past work, the most common UAV model involves only speed and heading as the states to be controlled, and motion occurs in the horizontal-plane with an independent altitude control system [14-16]. A two-dimensional approach for spacecraft maneuvers is insufficient, however, as the relative motion is quite inherently three-dimensional.

Furthermore, LVFs have been used primarily for limit-cycle motion, with the UAV 
converging to a circular or distorted-circular path [14-17] in an inertial or translating reference frame. The docking problem, however, is not a limit-cycle motion.

By far the most important distinction, however, is the requirement of spacecraft to track trajectories which are defined with respect to rotating or tumbling reference frames. To date, nearly all applications of LVFs include only trajectories defined within stationary or translating reference frames. This distinction is very important for the docking application, because trajectories defined with respect to tumbling reference frames will significantly complicate meeting the acceleration constraints.

As the only two (to the authors knowledge) notable exceptions, [18] and [19] both include trajectories in rotating reference frames. The methods for rotation compensation in these papers, however, are quite reliant on significant dynamics or initial condition assumptions. In the work by Scorsoglio and Furfaro [18] for example, the reference frame rotates synchronously with a spacecraft's orbit, and therefore, the authors assume that the rotation of the reference frame can be fully tracked regardless of the chaser vehicles distance to the origin. That is, there is an underlying assumption regarding a limit to the reference frame rotation rate. In work by Hough and Ulrich [19], the reference frame in consideration is fixed to a tumbling spacecraft, and rotates at arbitrarily high rates. To avoid thruster saturations from tracking this rotating reference frame, the authors therefore assume that the chaser must start sufficiently close to the target.

Presently, there exists no method in the framework of LVFs to track a trajectory defined in a rotating reference frame if the rotation can be arbitrarily fast and the two spacecraft can be separated by arbitrary distances. Furthermore, LVFs do not explicitly include fuel optimization, which is one of the essential criterion identified for any practical docking algorithm.

\subsection{Previous Work}

Lawrence [14] first introduced LVFs for standoff stationary-target tracking, and a general mathematical construct for deriving simple three-dimensional position-based LVFs for stand-off tracking was later presented by Lawrence et al. [17]. Beyond this general construction, [17] also presented some expanded LVF capabilites compared to 
previous applications, such as the ability to track "warped-circular" patterns, and a switching algorithm for way-point guidance. This work was then expanded upon by Frew et al. [15] for two UAVs performing phase-shifted cooperative stand-off tracking of a moving ground vehicle with perturbations from wind.

Several authors have studied how the work by $[14,15,17]$ can be altered to bound the curvature of flight paths. For example, Pothen and Ratnoo [16] proposed and analyzed a curvature parameter which is a function of radial distance, causing a more direct path with faster convergence and bounded curvature characteristics both inside and outside of the stand-off radius. Extending upon the work by [16], Sun et al. [20] proposed more complex variations on the variable curvature parameter to further reduce convergence time. To guarantee the turn rate of the UAV was respected, the authors proposed performing an offline search to numerically select the curvature parameter which minimized convergence time without violating the maximum turn rate. Very recent work by Che et al. [21] has further studied cooperative standoff tracking of moving targets (similar to the problem studied in [15]) in combination with curvature analysis similar to [16] and [20]. Specifically, [21] partially focused on optimizing the curvature parameter for minimum convergence time with a different algorithm compared to [20]. Moreover, [21] better defined the target state-estimation subsystem of the UAV flock, and an interacting multiple model-based unscented Kalman filter was proposed. There are far more examples of papers studying variants of the original standoff tracking LVFs proposed by [14]- [15] which can be found in the literature reviews of each of the papers mentioned above. While the problem of UAV standoff tracking has clearly been studied extensively, some authors have expanded LVFs beyond this application. For example, LVFs and other closely-related vector fields have been used for UAV obstacle avoidance $[22,23]$.

Scorsoglio and Furfaro [18] used a LVF-based relative motion guidance for docking in a cislunar space environment. Quite uniquely, [18] uses an Extreme Learning Machine (ELM) approach, such that the controlled spacecraft learns a LVF which can perform docking while meeting thrust constraints and avoiding obstacles. Because an artificial intelligence approach is taken, the authors are also able to include fuel optimizations, which is not conventionally considered with LVFs, but is essential for 
spacecraft applications. One important assumption used in [18], however, is that the target spacecraft is well-controlled, and therefore has a constant attitude with reference to a slowly rotating orbital reference frame. To compensate for the rotation

of this reference frame without violating thrust constraints, the authors include the slowly evolving nonlinear dynamics equations directly in their training. However, it is unclear whether this method of training thrust-limitation by inclusion of known reference-frame dynamics can be extended to include the tumbling of a spacecraft with arbitrary inertial properties and initial conditions.

In work by Hough and Ulrich [19], a different approach is taken to the docking problem by developing a LVF for a tumbling target based on body-fixed coordinates. The two spacecraft are assumed to have been placed in nearly identical orbits by the time the docking procedure begins, and therefore the acceleration differences due to gravity are considered negligible compared to the control inputs. It is assumed that the chaser spacecraft can obtain basic information about the target through observation, such as the maximum angular velocity and angular acceleration norms. Through a classical Lyapunov construction, asymptotic stability is guaranteed to the docking port. Two parameters (namely, the maximum relative velocity and a radius of application) are found such that, when distance to the docking port is less than the radius of application, the combined trajectory and compensation of the rotating reference frame cannot exceed the chaser thrust limitations. Outside this radius, the chaser simply ignores the attitude of the target and contracts inwards at constant speed. Clearly, the behaviour inside and outside of the radius of application represent two different guidance modes. However, it is concluded that there does not presently exist any obvious (and low computation) method to transition between these two modes while still meeting acceleration constraints and guaranteeing stability.

\subsection{Thesis Objectives}

The primary novel contributions of this thesis are the following: 
- A novel extension to LVFs is presented (herein referred to as a "cascaded Lyapunov vector field) which is suitable for tracking trajectories defined in rotating/tumbling reference frames from arbitrary distances while respecting acceleration constraints;

- the CLVF and LVF methods are combined to solve the docking problem in a two-phase maneuver;

- finally, the new docking method is validated in simulations and in experiment.

Furthermore, to increase the practicality of the maneuver, a strategy for fuel optimization is presented. Specifically:

- a method for roughly estimating the time and fuel usage of each maneuver phase is developed based on first principles;

- based on maneuver time estimates, fuel estimates, and constraints derived in this work to guarantee a given acceleration bound, a convex optimization is formulated to optimize each maneuver phase according to the desired performance.

Note that the attitude dynamics of the controlled spacecraft are assumed to be controlled by a separate subsystem, and therefore, only the translational docking problem is considered here. This assumption is valid for the vast majority of real spacecraft. Moreover, it will be assumed that the controlled spacecraft does not require use of the thrusters for purposes of attitude control during the docking maneuver. This assumption is again very practical, as nearly all modern spacecraft make use of independent actuators (such as reaction wheels) when active three-axis attitude control is required $[24,25]$. It is only during processes such as momentum dumping where use of the spacecraft thrusters may be required for attitude control. It is assumed that the docking procedure would not initiate if the reaction wheels were soon in need of momentum dumping to prevent saturation. 


\subsection{Organization}

The remainder of this work is organized as follows: Chapter 2 reviews the necessary theory from existing LVF papers, and provides a very typical design example for UAV standoff tracking of a stationary target. Then, Chapter 3 develops the theory of cascaded Lyapunov vectors fields (CLVFs) to extend the capabilities of LVFs to be suitable for convergence to a trajectory defined in a rotating or tumbling reference frame, which is necessary for spacecraft applications. Chapter 4 presents the scenario dynamics, and introduces the docking problem as a two-phase maneuver. The individual guidance and control laws for each phase are then developed in Chapter 5. Furthermore, a parameter optimization technique is developed for the docking problem in Chapter 5 as a method to include fuel optimization in the CLVF-LVF framework. The resulting algorithms are then tested in simulation in Chapter 6, and validated in experiment in Chapter 7. Finally, conclusions and future research directions are given in Chapter 8. 


\section{Chapter 2}

\section{Review of Lyapunov Vector Fields Theory}

For completeness, this Chapter reviews the theory and use of LVFs to date. Specifically, Sec. 2.1 will review the fundamental-level theory of LVFs, and Sec. 2.2 will review a specific design case for a UAV performing stand-off tracking on a stationary target. This review is designed to familiarize the reader with LVFs and is not intended to be comprehensive beyond what is necessary for further developments. More details on alternative uses of LVFs can be found in the works referenced in the literature review (Sec. 1.3).

\subsection{General Lyapunov Vector Field Theory}

Consider a dynamic closed-loop system

$$
\dot{\boldsymbol{x}}=f(\boldsymbol{x})
$$

where $\boldsymbol{x} \in \mathbb{R}^{n}$ is a state vector. Additionally, design a Lyapunov function of the states given by $V(\boldsymbol{x}) \in \mathbb{R}$ where it is required that $V \geq 0$. Note that the characteristics of this Lyapunov function are listed below, and a comprehensive introduction to Lyapunov stability is given in [26]. An attractor $\mathbb{A} \subset \mathbb{R}^{n}$ is defined as the set $\mathbb{A}=$ $\{\boldsymbol{x} \mid V(\boldsymbol{x})=0\}$. Note that the shape of $\mathbb{A}$ will be directly related to the shape of the final desired trajectory. For example, if the desired motion of the controlled vehicle about its target is circular, then one must design $V$ such that $\mathbb{A}$ forms a circle, with increasing "energy penalties" as the vehicle diverges from $\mathbb{A}$. To prove globally asymptotically stable guidance, there are four conditions of the Lyapunov function $V[17]$ :

A1) $V(\mathbb{A})=0$, otherwise $V(\boldsymbol{x})>0$

A2) $V$ is radially unbounded; as $\|\boldsymbol{x}\| \rightarrow \infty, V \rightarrow \infty$; 
A3) $V$ is continuously differentiable, and $\partial V / \partial \boldsymbol{x}$ is only $\mathbf{0}$ on within $\mathbb{A}$;

A4) $V$ is solely a function of $\boldsymbol{x}$ (i.e. not an explicit function of time).

After the Lyapunov function $V$ and corresponding attractor $\mathbb{A}$ have been defined, the construction of a LVF can be described by the following steps:

1. Define a component of the LVF in the negative gradient direction (this component also be multiplied by a positive definite matrix). This portion of the field is denoted $\boldsymbol{c}(\boldsymbol{x}, \cdot): \mathbb{R}^{n} \times \operatorname{Dom}(\cdot) \rightarrow \mathbb{R}^{n}$. Note that $(\cdot)$ is used for a stand-in for "any other variables", with $\operatorname{Dom}(\cdot)$ referring to their domain, and $\times$ in this case denotes the Cartesian product.

2. Define a second component which is perpendicular to the gradient direction. This component will not affect stability, but is responsible for the desired motion within $\mathbb{A}$. Denote this component as $\boldsymbol{s}(\boldsymbol{x}, \cdot): \mathbb{R}^{n} \times \operatorname{Dom}(\cdot) \rightarrow \mathbb{R}^{n}$

Expressing the steps above mathematically, we say that the $\operatorname{LVF} \boldsymbol{h}(\boldsymbol{x}, \cdot)$ is given by

$$
\boldsymbol{h}(\boldsymbol{x}, \cdot)=\boldsymbol{c}(\boldsymbol{x}, \cdot)+\boldsymbol{s}(\boldsymbol{x}, \cdot)
$$

with the constraints

B1)

$$
\boldsymbol{c}(\boldsymbol{x}, \cdot)=-\boldsymbol{\Gamma}(\boldsymbol{x}, \cdot) \frac{\partial V^{T}}{\partial \boldsymbol{x}}
$$

$\mathrm{B} 2)$

$$
\frac{\partial V}{\partial \boldsymbol{x}} \boldsymbol{s}(\boldsymbol{x}, \cdot)=0
$$

B3)

$$
\Gamma(\boldsymbol{x}, \cdot) \in \mathbb{R}^{n \times n} \text { is a positive definite matrix with respect to the set } \mathbb{A}
$$


What is meant by B3) is that $\boldsymbol{\Gamma}(\boldsymbol{x}, \cdot)$ is symmetric, and the quadratic product

$$
q=(\boldsymbol{x}-\mathbb{A})^{T} \boldsymbol{\Gamma}(\boldsymbol{x}, \cdot)(\boldsymbol{x}-\mathbb{A})
$$

is positive everywhere, except for when $\boldsymbol{x} \in \mathbb{A}$ where $q=0$. In this product, $(\boldsymbol{x}-\mathbb{A})$ is used to represent the shortest vector connecting the attractor $\mathbb{A}$ to the point $\boldsymbol{x}$. Asymptotic stability of $\boldsymbol{x}$ to the set $\mathbb{A}$ can then be guaranteed by the following straight-forward proof, given in several of the references $[16,17]$.

Theorem 1. Under the LVF guidance given by Eq. (2.2) and constraints A1)-A4) and B1)-B3), the state vector $\boldsymbol{x}$ is globally asymptotically stable to the set $\mathbb{A}$.

Proof. Given condition A4), the time-derivative of $V$ is found by

$$
\dot{V}=\frac{\partial V}{\partial \boldsymbol{x}} \dot{\boldsymbol{x}}
$$

By assuming perfect tracking of the trajectory described by the LVF

$$
\dot{V}=\frac{\partial V}{\partial \boldsymbol{x}}(\boldsymbol{c}(\boldsymbol{x}, \cdot)+\boldsymbol{s}(\boldsymbol{x}, \cdot)) .
$$

Using constraints B1) and B2), this simplifies to

$$
\dot{V}=-\frac{\partial V}{\partial \boldsymbol{x}} \boldsymbol{\Gamma}(\boldsymbol{x}, \cdot) \frac{\partial V^{T}}{\partial \boldsymbol{x}}
$$

Constraints A1)-A3) and B3) then complete the proof.

To track the LVF, the following "acceleration" controller is proposed by Lawrence et al. [17]

$$
\ddot{\boldsymbol{x}}(\boldsymbol{x}, \boldsymbol{h}, \cdot)=-\beta(\dot{\boldsymbol{x}}-\boldsymbol{h})+\dot{\boldsymbol{h}}
$$

where $\beta \in \mathbb{R}$ is a strictly positive parameter. Asymptotic tracking of the LVF is then given by the following

Theorem 2. Under the acceleration law given by Eq. (2.7), the time-derivative of the controlled states, $\dot{\boldsymbol{x}}$, is globally asymptotically convergent to the $L V F, \boldsymbol{h}(\boldsymbol{x}, \cdot)$. 
Proof. To begin, we define an error Lyapunov function $W$ as

$$
W=\frac{(\dot{\boldsymbol{x}}-\boldsymbol{h})^{T}(\dot{\boldsymbol{x}}-\boldsymbol{h})}{2}
$$

which clearly meets A1)-A4) where tracking error is the state. A time-derivative of $W$ then results in

$$
\dot{W}=(\dot{\boldsymbol{x}}-\boldsymbol{h})^{T}(\ddot{\boldsymbol{x}}-\dot{\boldsymbol{h}})
$$

and substitution of Eq. (2.7) results in

$$
\dot{W}=-\beta(\dot{\boldsymbol{x}}-\boldsymbol{h})^{T}(\dot{\boldsymbol{x}}-\boldsymbol{h})
$$

which completes the proof.

\subsection{Lyapunov Vector Fields for Unmanned Air Vehicles}

A design example for UAV standoff tracking of a stationary target will now be presented (more details on this design case can be found in [14-17]). To begin, let us define a normal vector of the desired circular trajectory $\hat{\boldsymbol{n}} \in \mathbb{R}^{3}$. Note that throughout this work, the symbol $\{\hat{\bullet}\}$ will be used to denote a unit-vector; in some specific cases, the "hat" symbol is used to denote an "estimated value", but this will be clear from the context.

Next, the controlled state is selected as the position vector $\boldsymbol{r} \in \mathbb{R}^{3}$, and $\boldsymbol{r}=\mathbf{0}$ is defined to be directly above the target at the desired altitude. A Lyapunov function on which to construct the LVF must now be selected. One option is [17]

$$
V(\boldsymbol{r})=\frac{1}{2}\left(\boldsymbol{r}_{n}^{T} \hat{\boldsymbol{r}}_{n}\right)^{2}+\frac{1}{2}\left(\boldsymbol{r}_{t}^{T} \hat{\boldsymbol{r}}_{t}-\eta\right)^{2}
$$

where $\eta \in \mathbb{R}>0$ is the desired circular radius, and subscripts $t$ and $n$ denote the tangent and normal components with reference to the normal vector $\hat{\boldsymbol{n}}$. That is

$$
\begin{gathered}
\mathbf{r}_{n}=\hat{\boldsymbol{n}} \hat{\boldsymbol{n}}^{T} \boldsymbol{r} \\
\mathbf{r}_{t}=\left(\boldsymbol{I}-\hat{\boldsymbol{n}} \hat{\boldsymbol{n}}^{T}\right) \boldsymbol{r}
\end{gathered}
$$


where $\boldsymbol{I}$ will be used in this work to mean the identity matrix of appropriate dimension. Note that $V=0$ if and only if $r_{n}=0$ and $r_{t}=\eta$, defining the attractor $\mathbb{A}$ (it will be common notation to use non-bold letters to refer to norms, i.e. $r_{n}=\left\|\boldsymbol{r}_{n}\right\|$ and $\left.r_{t}=\left\|\boldsymbol{r}_{t}\right\|\right)$. Analyzing Eq. (2.11) it is clear that the conditions A1) - A4) are met. Equation (2.11) can be used to build the following guidance law

$$
\boldsymbol{h}(\boldsymbol{r})=-\boldsymbol{\Gamma}(\boldsymbol{r}) \frac{\partial V^{T}}{\partial \boldsymbol{r}}+\boldsymbol{s}(\boldsymbol{r})
$$

where $\boldsymbol{h}(\boldsymbol{r})$ is the desired velocity, $\boldsymbol{\Gamma}(\boldsymbol{r})$ is positive definite (and often a multiple of the identity) matrix, and $\boldsymbol{s}(\boldsymbol{r})$ is the circulation term, as it will cause the UAV to fly in a circle upon arrival to the attractor. Options for $\boldsymbol{\Gamma}(\boldsymbol{r})$ and $\boldsymbol{s}(\boldsymbol{r})$ which keep the UAV flying at a constant speed are

$$
\begin{aligned}
& \boldsymbol{\Gamma}(\boldsymbol{r})=\frac{1}{\alpha(\boldsymbol{r})} \boldsymbol{I} \\
& \boldsymbol{s}(\boldsymbol{r})=\gamma \frac{\hat{\boldsymbol{n}}^{\times} \boldsymbol{r}_{t}}{\alpha(\boldsymbol{r})}
\end{aligned}
$$

where $\gamma \in \mathbb{R}$ denotes a curvature parameter (well studied in $[16,20,21]$ ), $\{\cdot\}^{\times}$denotes the skew-symmetric construction of the vector $\{\cdot\}$ and is used for computing crossproducts when a vector has known coordinates, and $\alpha$ is a normalization parameter given by

$$
\alpha(\boldsymbol{r})=\frac{1}{v}\left(r_{n}^{2}+\left(r_{t}-\eta\right)^{2}+r_{t}^{2} \gamma^{2}\right)^{1 / 2}
$$

where $v \in \mathbb{R}>0$ is the constant desired speed. By shifting parameters such as $\gamma$ or $v$, various "shapes" of LVF can be formed, resulting in varying trajectories towards and around the desired circle of radius $\eta$ as seen in Fig. 2.1. 


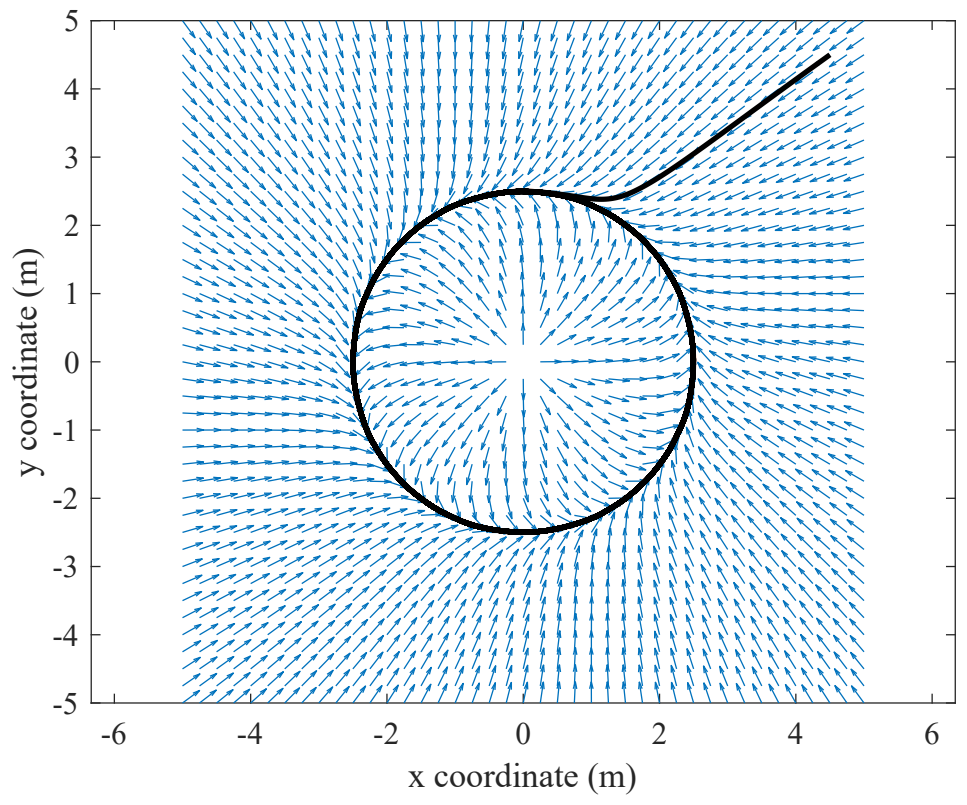

(a) Lyapunov vector field with $\gamma=0.1$.

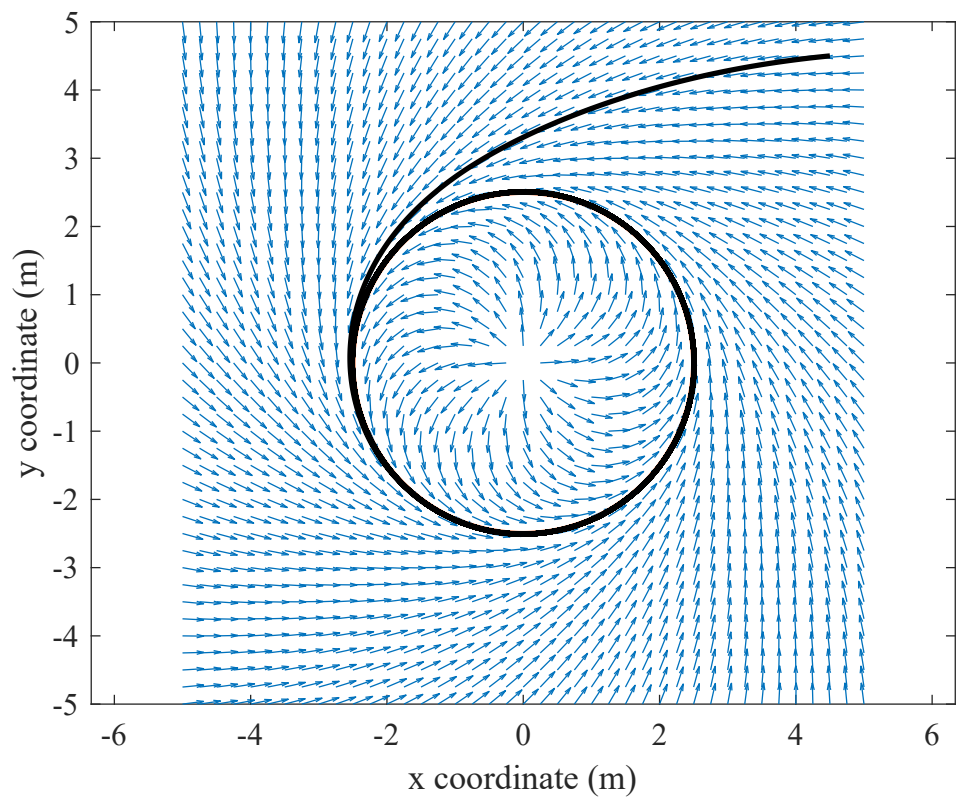

(b) Lyapunov vector field with $\gamma=0.5$.

Figure 2.1: Lyapunov vector fields with $\eta=3, \hat{\boldsymbol{n}}$ in vertical direction. 


\section{Chapter 3}

\section{Development of Cascaded Lyapunov Vector Fields}

An extension to Lyapunov vector fields is presented for tracking trajectories within rotating (or generally, tumbling) and accelerating reference frames. This extension is computationally-light, and is acceleration- rather than velocity-constrained, making it suitable for real-time use in spacecraft. A general stability analysis proves globally asymptotic stability given a set of conditions which are analogous to those of standard Lyapunov vector fields. A special case of the cascaded Lyapunov vector field (CLVF) is closely studied, and a simple set of conditions guaranteeing bounded acceleration commands for perfect tracking are derived. A design procedure for the new guidance law is presented. Finally, a full design example for a spacecraft proximity inspection mission is presented. The simulations demonstrate that the cascaded Lyapunov vector field is able to guide an acceleration-constrained vehicle from an inertial reference frame into a tumbling body-fixed trajectory while respecting acceleration constraints. Futhermore, no computationally expensive in-the-loop algorithms are required to meet constraints.

\subsection{Problem Formuation}

In this Section, the problem of tracking LVFs in generally rotating and translating reference frames is introduced. Specifically, Sec. 3.1 .1 will introduce the reference frames and vector definitions used for this Chapter. Then, Sec. 3.1.2 provides some motivation for why the present LVF theory is not well suited for tracking in generally rotating and translating reference frames, and introduces some intuition for cascading two LVFs to solve this problem. 


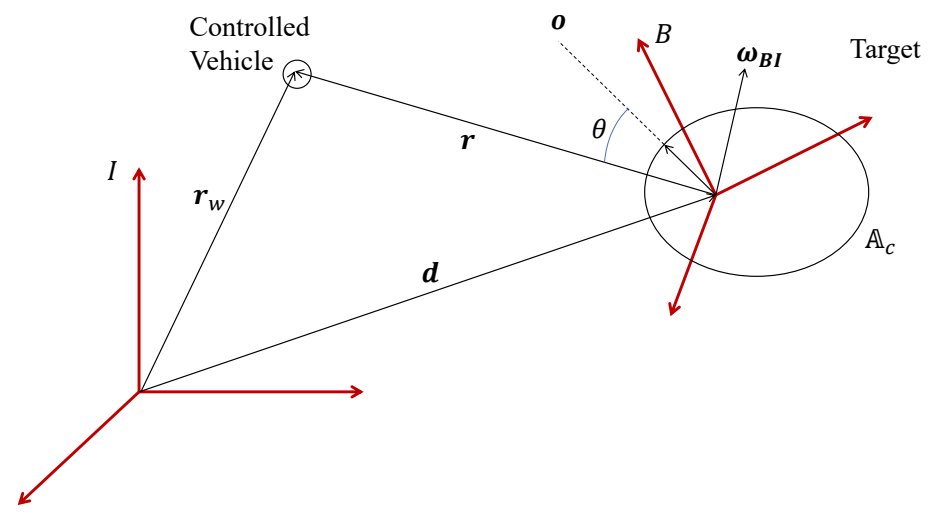

Figure 3.1: Reference frame and vector definitions.

\subsubsection{Reference Frame and Vector Definitions}

To begin, define a body-fixed reference frame $B$ which is fixed to the target position and orientation. Also define an inertial world reference frame $I$. The origin of $B$ relative to the origin of $I$ is given by the vector $\boldsymbol{d}(t) \in \mathbb{R}^{3}$. The controlled vehicle position relative to the origin of $I$ is denoted $\boldsymbol{r}_{w}(t) \in \mathbb{R}^{3}$, and relative to the origin of $B$ is denoted $\boldsymbol{r}(t) \in \mathbb{R}^{3}$ (i.e. $\boldsymbol{r}(t)=\boldsymbol{r}_{w}(t)-\boldsymbol{d}(t)$ ). The rotation matrix which converts $B$ coordinates into $I$ coordinates is given by $\boldsymbol{C}_{I B}(t) \in \mathbb{R}^{3 \times 3}$. Finally, the angular velocity of $B$ relative to $I$ is exactly equal to the angular velocity of the rigid target, and is given by $\boldsymbol{\omega}_{B I}(t)$. There is an attractor $\mathbb{A}_{c}$ which is completely stationary when viewed from $B$.

The desired position within the attractor is described by a single time-varying vector $\boldsymbol{o}(t) \in \mathbb{R}^{3}$ which, by definition, must have a magnitude such that the tip of the vector is on the surface $\mathbb{A}_{c}$. Lastly, let us define the angle between $\boldsymbol{r}(t)$ and $\boldsymbol{o}(t)$ as $\theta(t) \in[0, \pi]$. A diagram of these definitions is given in Fig. 3.1.

For writing equations, it will always be assumed that the vector is being expressed in coordinates defined by some reference frame. When this reference frame is not inertial, a superscript will denote the reference frame being used. For example, if the vector relationship for three arbitrary vectors $\boldsymbol{\zeta}_{1} \in \mathbb{R}^{3}, \boldsymbol{\zeta}_{2} \in \mathbb{R}^{3}$ and $\boldsymbol{\zeta}_{3} \in \mathbb{R}^{3}$ in 
inertial coordinates is given by

$$
\zeta_{1}=\zeta_{2}+\zeta_{3}
$$

then this relationship can also be written as addition in a reference frame $B$ followed by a rotation into the inertial frame as

$$
\zeta_{1}=C_{I B}\left(\zeta_{2}^{B}+\zeta_{3}^{B}\right)
$$

Likewise, the additive property of angular velocities means that for some arbitrary reference frame $F$ which rotates with respect to $B$, the total angular velocity about the inertial frame can be computed as

$$
\boldsymbol{\omega}_{F I}=\boldsymbol{\omega}_{F B}+\boldsymbol{\omega}_{B I}
$$

or, as in most practical cases where $\boldsymbol{\omega}_{B I}$ is measured in the reference frame $B$ and $\boldsymbol{\omega}_{F B}$ is measured in the reference frame $F$,

$$
\boldsymbol{\omega}_{F I}=\boldsymbol{C}_{I F}\left(\boldsymbol{\omega}_{F B}^{F}+\boldsymbol{C}_{F B} \boldsymbol{\omega}_{B I}^{B}\right)
$$

where $\boldsymbol{C}_{I F}$ is a rotation cosine matrix, which could further be expressed as

$$
C_{I F}=C_{I B} C_{B F}
$$

The time-derivative of some vector $\zeta \in \mathbb{R}^{3}$ as seen in some rotating reference frame $F$ will be denoted by $\dot{\boldsymbol{\zeta}}^{F}$. When solving for the inertial time-derivative $\dot{\boldsymbol{\zeta}}$, it is expressed

$$
\dot{\boldsymbol{\zeta}}=\boldsymbol{C}_{I F}\left(\boldsymbol{\omega}_{F I}^{\times} \boldsymbol{\zeta}^{F}+\dot{\boldsymbol{\zeta}}^{F}\right)
$$

where the skew-symmetric construction $\boldsymbol{\omega}_{F I}^{\times}$is assumed to be performed with coordinates in $F$, as it multiplies a vector expressed in $F$ (i.e. the superscript $F$ on $\boldsymbol{\omega}_{F I}^{\times}$ is dropped to avoid a double superscript notation, as the coordinate frame is already known from the context).

Non-bold letters will be used to represent scalars. For vector quantities, non-bold letters will be used to represent their norm. For example the norm of an arbitrary 
vector $\zeta$ is expressed

$$
\zeta=\|\zeta\|
$$

\subsubsection{Tracking Lyapunov Vector Fields in Rotating Reference Frames}

Suppose that the controlled vehicle has some desired trajectory defined in $B$. If LVFs are to be used, then the attractor $\mathbb{A}_{c}$ of course must be defined as a constant set of coordinates in $B$, and the desired trajectory must move through $\mathbb{A}_{c}$. If the rotation is slow enough (such is the case in [18]), it may be reasonable to define a LVF directly in $B$. That is, the the guidance would be defined with $B$ coordinates such that

$$
\boldsymbol{h}^{B}=\boldsymbol{c}^{B}\left(\boldsymbol{r}^{B}\right)+\boldsymbol{s}^{B}\left(\boldsymbol{r}^{B}\right)
$$

where $\boldsymbol{c}^{B}\left(\boldsymbol{r}^{B}\right)$ and $\boldsymbol{s}^{B}\left(\boldsymbol{r}^{B}\right)$ meet B1)-B3) for some valid Lyapunov function of $\boldsymbol{r}^{B}$. The resulting guidance seen in the inertial reference frame is then attained as

$$
\boldsymbol{h}=\dot{\boldsymbol{d}}+\boldsymbol{C}_{I B}\left(\boldsymbol{\omega}_{B I}^{\times} \boldsymbol{r}^{B}+\boldsymbol{h}^{B}\right)
$$

and assuming perfect tracking takes place, the acceleration to track this field is given by

$$
\dot{\boldsymbol{h}}=\ddot{\boldsymbol{d}}+\boldsymbol{C}_{I B}\left(\boldsymbol{\omega}_{B I}^{\times} \boldsymbol{\omega}_{B I}^{\times} \boldsymbol{r}^{B}+\dot{\boldsymbol{\omega}}_{B I}^{\times} \boldsymbol{r}^{B}+2 \boldsymbol{\omega}_{B I}^{\times} \boldsymbol{h}^{B}+\dot{\boldsymbol{h}}^{B}\right)
$$

Immediately, there is the apparent issue that as $\|\boldsymbol{r}\| \rightarrow \infty,\|\dot{\boldsymbol{h}}\| \rightarrow \infty$ provided $\boldsymbol{\omega}_{B I} \not \equiv \mathbf{0}$ and that $\boldsymbol{\omega}_{B I}$ is not perfectly parallel to $\boldsymbol{r}$. While the accelerations may be cancelled for sufficiently slow rotation, for convergence to a rotating vehicle this method will quickly saturate thrusters. One method to avoid this saturation is to constrain the LVF to apply only within a limited radius. However, this approach simply pushes the problem further back. That is, if the initial position is outside of the cut-off radius, then the new problem becomes finding a method to enter the cut-off radius such that: acceleration limits are not violated, and the target attitude is perfectly tracked just as the cut-off radius is entered.

The intuitive idea of CLVFs is that one LVF-like guidance "contracts inertially" from arbitrarily far away, while a second LVF-like guidance gradually transitions to 
track the target attitude in a manner which guarantees bounded acceleration commands. The groundwork for such an approach, including general statements regarding stability and acceleration constraints, is the purpose of this Chapter. Herein, the field responsible for contraction is referred to as the "contraction field", and the field responsible for tracking the body-fixed trajectory through $\mathbb{A}_{c}$ is referred to as the "alignment field".

\subsection{General Cascaded Lyapunov Vector Fields: Structure and Stability}

The structure of the CLVF is introduced in Sec. 3.2.1, and a set of constraints required for stability which are analogous to B1)-B3) are presented. Then, the stability under these new analogous constraints is analyzed in Sec. 3.2.2. It is shown that the contraction field is asymptotically stable, and that the alignment field becomes asymptotically stable as a disturbance term is driven to 0 by the contraction field. In Sec. 3.2.3, assumptions which are broadly applicable to LVF applications are used to show that the new analogous stability conditions are usually not difficult to satisfy. Finally, a study is performed in Sec. 3.2.4 to analyze the robustness of the alignment field asymptotic stability in cases where the disturbance term is not driven perfectly to 0 .

\subsubsection{Basic Structure of the Cascaded Lyapunov Vector Field}

To begin, let us define a Lyapunov function $V_{c}$ which meets A1)-A4) for the state $\boldsymbol{r}^{B}$. This must be the case, as it is desired that the attractor of $V_{c}$ rotates with the vehicle attitude (i.e. the shape of the attractor may be driven by the shape of the target vehicle). An equivalent statement is that $V_{c}$ is a function of $\boldsymbol{r}$ in inertial coordinates and the orientation $B$ with respect to $I$. A rotation of the contours of $V_{c}$ while keeping $\boldsymbol{r}$ constant is illustrated in Fig. 3.2.

The CLVF is given the familiar high-level structure

$$
\boldsymbol{h}(\boldsymbol{r}, \cdot)=\boldsymbol{c}(\boldsymbol{r}, \cdot)+\boldsymbol{s}(\boldsymbol{r}, \cdot)+\dot{d}
$$

where it is assumed that the controlled vehicle is easily capable of tracking the target 


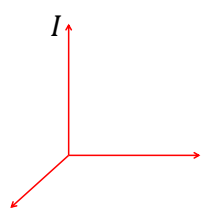

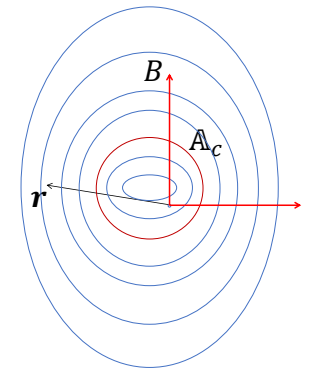

(a) Orientation 1.
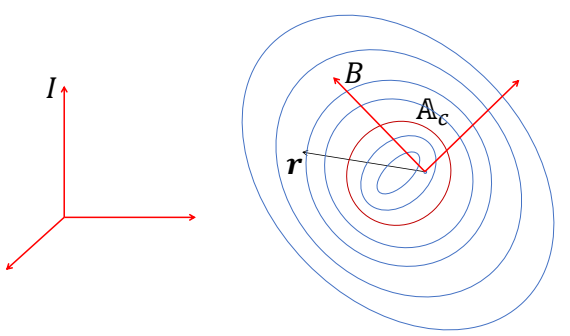

(b) Orientation 2 .

Figure 3.2: $V_{c}$ contours in two different orientations.

vehicle velocity $\dot{\boldsymbol{d}}$. As is normally the case, the contraction component $\boldsymbol{c}$ is subject to B1)

$$
\boldsymbol{c}(\boldsymbol{r}, \cdot)=-\boldsymbol{\Gamma}_{c}(\boldsymbol{r}, \cdot) \frac{\partial V_{c}^{T}}{\partial \boldsymbol{r}}
$$

where $\boldsymbol{\Gamma}_{c}$ is a positive definite matrix to an attractor $\mathbb{A}_{c}$ defined by $V_{c}$. The term $\boldsymbol{s}(\boldsymbol{r}, \cdot)$, however, is not subject to B2), but rather is subject to the constraint

$$
\frac{\partial V_{c}}{\partial \boldsymbol{r}} \boldsymbol{s}(\boldsymbol{r}, \cdot)=-\frac{\partial V_{c}}{\partial \boldsymbol{q}_{B I}} \dot{\boldsymbol{q}}_{B I}
$$

where $\boldsymbol{q}_{B I} \in \mathbb{R}^{p}$ is some vector of parameters characterizing the attitude of $B$ with respect to $I$ for some appropriate integer $p$ (dependent on the representation choice). Note that if the frame $B$ does not rotate, or if $V_{c}$ is invariant to rotation of $B$, then Eq. (3.13) reduces to B2). Therefore, for either of these special cases a LVF is formed on the Lyapunov function $V_{c}$, and the controlled vehicle will be globally asymptotically stable onto $\mathbb{A}_{c}$. Let us further depart from standard LVFs by defining a desired trajectory within $\mathbb{A}_{c}$ and provide the term $\boldsymbol{s}(\boldsymbol{r}, \cdot)$ with the following structure

$$
\boldsymbol{s}(\boldsymbol{r}, \cdot)=\boldsymbol{a}(\boldsymbol{r}, \boldsymbol{o}, \cdot)+\boldsymbol{g}(\boldsymbol{r}, \dot{\boldsymbol{o}}, \cdot)
$$

where $\boldsymbol{g}: \mathbb{R}^{3} \times \mathbb{R}^{3} \times \operatorname{Dom}\{\cdot\} \rightarrow \mathbb{R}^{3}$ is a velocity function responsible for tracking the attitude of the reference frame $B$, and $\boldsymbol{a}: \mathbb{R}^{3} \times \mathbb{R}^{3} \times \operatorname{Dom}\{\cdot\} \rightarrow \mathbb{R}^{3}$ is a function 
referred to as the "alignment" component of velocity with the structure

$$
\boldsymbol{a}(\boldsymbol{r}, \boldsymbol{o}, \cdot)=-\boldsymbol{\Gamma}_{a}(\boldsymbol{r}, \boldsymbol{o}, \cdot) \frac{\partial V_{a}^{T}}{\partial \boldsymbol{r}}
$$

where $V_{a}$ is a Lyapunov function of $\theta$ meeting constraints A1), A3) and A4), and the single desired trajectory point $\boldsymbol{o}(t)$ defines the attractor of $V_{a}$. Note that $V_{a}$ does not meet A2) as $\theta$ exists in a bounded set $[0, \pi]$ and therefore does not require global stability properties. The matrix $\Gamma_{a} \in \mathbb{R}^{3 \times 3}$ is not necessarily positive-definite, but must be positive semi-definite. Specifically, $\boldsymbol{\Gamma}_{a} \in \mathbb{R}^{3 \times 3}$ can be designed such that

$$
\lim _{\theta \rightarrow 0} \boldsymbol{a}(\boldsymbol{r}, \boldsymbol{o}, \cdot)=\lim _{\theta \rightarrow \pi} \boldsymbol{a}(\boldsymbol{r}, \boldsymbol{o}, \cdot)=\mathbf{0}
$$

and therefore can be singular everywhere along the line $\boldsymbol{r}$ where $\theta=0$ or (equivalently) the line of $\boldsymbol{r}$ where $\theta=\pi$, and is otherwise strictly positive definite. As will become apparent later, this trait is useful for constraining acceleration. Intuitively, this means that for the case of maximum misalignment where $\theta \approx \pi$, the controlled vehicle will "wait" until the target has rotated such that $\theta$ is sufficiently far from $\pi$. Therefore, the CLVF will tend to choose alignment directions which oppose the rotation direction of the target, saving fuel. Substituting Eqs. (3.14) and (3.15) into Eq. (3.13), it is apparent that the following condition must be satisfied for stability

$$
\frac{d V_{c}}{d \boldsymbol{r}} \boldsymbol{\Gamma}_{a}(\boldsymbol{r}, \boldsymbol{o}, \cdot) \frac{\partial V_{a}^{T}}{\partial \boldsymbol{r}}=\frac{d V_{c}}{d \boldsymbol{r}} \boldsymbol{g}(\boldsymbol{r}, \dot{\boldsymbol{o}}, \cdot)+\frac{\partial V_{c}}{\partial \boldsymbol{q}_{B I}} \dot{\boldsymbol{q}}_{B I}
$$

Lastly, let us require that when $\boldsymbol{r}$ is in $\mathbb{A}_{c}$, the function $\boldsymbol{g}$ has the following constraint

$$
\left.\frac{\partial V_{a}}{\partial \boldsymbol{r}} \boldsymbol{g}(\boldsymbol{r}, \dot{\boldsymbol{o}}, \cdot)\right|_{\boldsymbol{r} \in \mathbb{A}_{c}}=-\frac{\partial V_{a}}{\partial \boldsymbol{o}} \dot{\boldsymbol{o}}
$$

At this point, it is beneficial to note some properties of the CLVF structure

1. Equation (3.11) resembles a LVF, and Eq. (3.14) also resembles a LVF. Indeed, Eq. (3.12) is the contraction field, and Eq. (3.14) is the alignment field. Note that the alignment field is embedded within the contraction field. 
2. The only term which relates to the trajecory $\dot{\boldsymbol{o}}(t)$ within $\mathbb{A}_{c}$ is $\boldsymbol{g}(\boldsymbol{r}, \dot{\boldsymbol{o}}, \cdot)$. Therefore, the function $\boldsymbol{g}(\boldsymbol{r}, \dot{\boldsymbol{o}}, \cdot)$ can be selected such that at large distances it is close to $\mathbf{0}$ and the target rotation is effectively ignored. When converging onto $\mathbb{A}_{c}$, the function $\boldsymbol{g}(\boldsymbol{r}, \dot{\boldsymbol{o}}, \cdot)$ must change to meet Eq. (3.18), which will ensure perfect tracking of the target attitude.

In summary, the set of stability conditions for CLVFs analogous to B1)-B3) for standard LVFs are as follows:

C1)

$$
\boldsymbol{c}(\boldsymbol{r}, \cdot)=-\boldsymbol{\Gamma}_{c}(\boldsymbol{r}, \cdot) \frac{\partial V_{c}^{T}}{\partial \boldsymbol{r}}
$$

C2)

$$
\frac{\partial V_{c}}{\partial \boldsymbol{r}} \boldsymbol{s}(\boldsymbol{r}, \cdot)=-\frac{\partial V_{c}}{\partial \boldsymbol{q}_{B I}} \dot{\boldsymbol{q}}_{B I} \text { or the equivalent statement given by Eq. }
$$

C3)

$$
\boldsymbol{\Gamma}_{c}(\boldsymbol{r}, \cdot) \text { is positive definite on } \mathbb{A}_{c}
$$

C4)

$$
\boldsymbol{a}(\boldsymbol{r}, \boldsymbol{o}, \cdot)=-\boldsymbol{\Gamma}_{a}(\boldsymbol{r}, \boldsymbol{o}, \cdot) \frac{\partial V_{a}^{T}}{\partial \boldsymbol{r}}
$$

C5)

$$
\left.\frac{\partial V_{a}}{\partial \boldsymbol{r}} \boldsymbol{g}(\boldsymbol{r}, \dot{\boldsymbol{o}}, \cdot)\right|_{\boldsymbol{r} \in \mathbb{A}_{c}}=-\frac{\partial V_{a}}{\partial \boldsymbol{o}} \dot{\boldsymbol{o}}
$$

C6)

$\boldsymbol{\Gamma}_{a}(\boldsymbol{r}, \boldsymbol{o}, \cdot)$ can allow $\lim _{\theta \rightarrow 0} \boldsymbol{a}(\boldsymbol{r}, \boldsymbol{o}, \cdot)=\lim _{\theta \rightarrow \pi} \boldsymbol{a}(\boldsymbol{r}, \boldsymbol{o}, \cdot)=\mathbf{0}$, but is strictly positive definite otherwise. 
While this list may seem long, note that every condition is in some way a variant on the familiar LVF conditions B1)-B3), and that there are six conditions only because there are two LVF-like structures within the CLVF.

\subsubsection{Stability}

The stability of the proposed CLVF will be proven in two steps. First, global asymptotic stability of $\boldsymbol{r}$ to $\mathbb{A}_{c}$ will be proven in Theorem 3. Then, using the results of Theorem 3 asymptotic stability of $\theta \rightarrow 0$ will be proven.

Theorem 3. Under the CLVF guidance given by Eq. (3.11) and constraints C1)-C3),

the vector $\boldsymbol{r}$ is globally asymptotically stable to the set $\mathbb{A}_{c}$ defined by the Lyapunov function $V_{c}$ which meets $\left.\left.A 1\right)-A 4\right)$ for the state vector $\boldsymbol{r}^{B}$.

Proof. Taking the time-derivative of $V_{c}$ with respect to inertial coordinates

$$
\dot{V}_{c}=\frac{\partial V_{c}}{\partial \boldsymbol{r}} \dot{\boldsymbol{r}}+\frac{\partial V_{c}}{\partial \boldsymbol{q}_{B I}} \dot{\boldsymbol{q}}_{B I}
$$

substituting in the definition of $\boldsymbol{r}_{w}$

$$
\dot{V}_{c}=\frac{\partial V_{c}}{\partial \boldsymbol{r}}\left(\dot{\boldsymbol{r}}_{w}-\dot{\boldsymbol{d}}\right)+\frac{\partial V_{c}}{\partial \boldsymbol{q}_{B I}} \dot{\boldsymbol{q}}_{B I}
$$

when assuming perfect tracking, this leads to

$$
\dot{V}_{c}=\frac{\partial V_{c}}{\partial \boldsymbol{r}}(\boldsymbol{c}(\boldsymbol{r}, \cdot)+\boldsymbol{s}(\boldsymbol{r}, \cdot))+\frac{\partial V_{c}}{\partial \boldsymbol{q}_{B I}} \dot{\boldsymbol{q}}_{B I}
$$

Invoking $\mathrm{C} 2$ ) leads to

$$
\dot{V}_{c}=\frac{\partial V_{c}}{\partial \boldsymbol{r}} \boldsymbol{c}(\boldsymbol{r}, \cdot)
$$

Then, by invoking C1)

$$
\dot{V}_{c}=-\frac{\partial V_{c}}{\partial \boldsymbol{r}} \boldsymbol{\Gamma}_{c}(\boldsymbol{r}, \cdot) \frac{\partial V_{c}^{T}}{\partial \boldsymbol{r}}
$$


This can be rearranged as

$$
\dot{V}_{c}=-\frac{\partial V_{c}}{\partial \boldsymbol{r}^{B}}\left(\boldsymbol{C}_{B I} \boldsymbol{\Gamma}_{c}(\boldsymbol{r}, \cdot) \boldsymbol{C}_{I B}\right){\frac{\partial V_{c}^{T}}{\partial \boldsymbol{r}^{B}}}^{T}
$$

Note that the matrix product between the brackets retains the positive definiteness of $\boldsymbol{\Gamma}_{c}(\boldsymbol{r}, \cdot)$. That is, pre-multiplication by a rotation matrix, followed by postmultiplication of the inverse rotation, only changes the reference frame in which the product is performed, and does not affect the positive-definiteness of $\boldsymbol{\Gamma}_{c}(\boldsymbol{r}, \cdot)$. The constraints A1)-A4) and C3) then complete the proof.

Remark. Note that the effect of C2) on $\boldsymbol{s}(\boldsymbol{r}, \cdot)$ is to keep $\boldsymbol{r}$ within the same contour of $V_{c}$. The added effect of $\boldsymbol{c}(\boldsymbol{r}, \cdot)$ is then to take $\boldsymbol{r}$ into a lower contour until $\mathbb{A}_{c}$ is reached.

Theorem 4. Under the CLVF guidance given by Eq. (3.11) and constraints C1)-C6), the vector $\boldsymbol{r}$ is asymptotically stable to the desired trajectory point $\boldsymbol{o}(t)$ which is the attractor for a Lyapunov function $V_{a}$ meeting A1), A3) and A4) for the state $\theta$.

Proof. Taking the time-derivative of $V_{a}$ gives

$$
\dot{V}_{a}=\frac{d V_{a}}{d \theta} \dot{\theta}
$$

or equivalently, using the fact that $\theta$ is a function of $\boldsymbol{r}$ and $\boldsymbol{o}$

$$
\dot{V}_{a}=\frac{\partial V_{a}}{\partial \boldsymbol{r}} \dot{\boldsymbol{r}}+\frac{\partial V_{a}}{\partial \boldsymbol{o}} \dot{\boldsymbol{o}}
$$

assuming perfect tracking, this leads to the expression

$$
\dot{V}_{a}=\frac{\partial V_{a}}{\partial \boldsymbol{r}}(\boldsymbol{c}(\boldsymbol{r}, \cdot)+\boldsymbol{a}(\boldsymbol{r}, \boldsymbol{o}, \cdot)+\boldsymbol{g}(\boldsymbol{r}, \dot{\boldsymbol{o}}, \cdot))+\frac{\partial V_{a}}{\partial \boldsymbol{o}} \dot{\boldsymbol{o}}
$$

Invoking $\mathrm{C} 1$ ) and $\mathrm{C} 4$ ) leads to

$$
\dot{V}_{a}=-\frac{\partial V_{a}}{\partial \boldsymbol{r}} \boldsymbol{\Gamma}_{a}(\boldsymbol{r}, \boldsymbol{o}, \cdot) \frac{\partial V_{a}^{T}}{\partial \boldsymbol{r}}+\delta(\boldsymbol{r})
$$


where $\delta(\boldsymbol{r})$ is a disturbance term given by

$$
\delta(\boldsymbol{r})=\left(\frac{\partial V_{a}}{\partial \boldsymbol{o}} \dot{\boldsymbol{o}}+\frac{\partial V_{a}}{\partial \boldsymbol{r}} \boldsymbol{g}(\boldsymbol{r}, \dot{\boldsymbol{o}}, \cdot)\right)-\frac{\partial V_{a}}{\partial \boldsymbol{r}} \boldsymbol{\Gamma}_{c}(\boldsymbol{r}, \cdot) \frac{\partial V_{c}^{T}}{\partial \boldsymbol{r}}
$$

note that independently of $V_{a}$, Theorem 3 mandates that $\boldsymbol{r} \rightarrow \mathbb{A}_{c}$. Also, noting C5) and the fact that $\frac{\partial V_{c}^{T}}{\partial \boldsymbol{r}}=\mathbf{0}$ on $\mathbb{A}_{c}$

$$
\delta\left(\mathbb{A}_{c}\right)=0
$$

Further, given C6), it can be said that for any $\theta$ that does not equal 0 or $\pi$, there is a contour of $V_{c}$ which will eventually be reached such that

$$
\left\|\frac{\partial V_{a}}{\partial \boldsymbol{r}} \boldsymbol{\Gamma}_{a}(\boldsymbol{r}, \boldsymbol{o}, \cdot) \frac{\partial V_{a}^{T}}{\partial \boldsymbol{r}}\right\|>\|\delta(\boldsymbol{r})\|
$$

because $\boldsymbol{r}$ will be sufficiently close to $\mathbb{A}_{c}$ and therefore $\delta(\boldsymbol{r})$ will be sufficiently close to 0 . At this point $\dot{V}_{a}$ will become negative, and therefore $\dot{V}_{a}$ is negative semi-definite in $\boldsymbol{r}$. To prove that $\dot{V}_{a}$ indeed becomes negative definite, let us show that $\frac{\partial V_{a}}{\partial \boldsymbol{r}}$ cannot become $\mathbf{0}$ anywhere except the points where $\theta=0$ or $\theta=\pi$. First, given that $V_{a}$ is a function of $\theta$ only, it is straight-forward to solve the Jacobian $\frac{\partial V_{a}}{\partial \boldsymbol{r}}$ as

$$
\frac{\partial V_{a}}{\partial \boldsymbol{r}}=\frac{d V_{a}}{d \theta} \frac{\partial \theta}{\partial \boldsymbol{r}}=\frac{d V_{a}}{d \theta}\left(\frac{\hat{\boldsymbol{r}}^{T} \cos \theta-\hat{\boldsymbol{o}}^{T}}{r \sin \theta}\right)
$$

Setting this Jacobian to $\mathbf{0}$ and rearranging, the following condition arises

$$
\hat{\boldsymbol{r}} \cos \theta=\hat{\boldsymbol{o}}
$$

which is impossible for any value of $\theta$ other than 0 or $\pi$. By looking at small perturbation angles $0+\Delta \theta$ and $\pi-\Delta \theta$ as $\delta(\boldsymbol{r}) \rightarrow 0$, it is clear that the point given by $\theta=\pi$ becomes an unstable equilibrium point, and that $\theta=0$ becomes an asymptotically stable equilibrium point as $\boldsymbol{r} \rightarrow \mathbb{A}_{c}$.

Remark. Note that, analogous to the stability proof to $\mathbb{A}_{c}$ from Theorem 3 , the effect of $C 5)$ on $\boldsymbol{g}(\boldsymbol{r}, \dot{\boldsymbol{o}}, \cdot)$ is to keep $\boldsymbol{r}$ within the same contour of $V_{a}$ once the $\mathbb{A}_{c}$ is reached. 
The added effect of the vector $\boldsymbol{a}(\boldsymbol{r}, \boldsymbol{o}, \cdot)$ is then to take $\boldsymbol{r}$ into a lower contour until $\boldsymbol{o}(t)$ is reached.

\subsubsection{Simplifying Assumptions to Satisfy Stability Conditions}

Dependent on the geometry of $\mathbb{A}_{c}$ and the contours of $V_{c}$, conditions $\mathrm{C} 2$ ) and C5) may be difficult to meet. Therefore, to simplify these conditions, some assumptions will be introduced which are very common in practical applications of LVFs. Namely, let us first assume that $\mathbb{A}_{c}$ is spherical with a radius $\alpha \in \mathbb{R}>0$, and that the origin of $B$ is centered within $\mathbb{A}_{c}$. Secondly, let us assume that $V_{c}$ is a function of the absolute distance $|r-\alpha|$ only.

Now, let us begin by simplifying the constraint C5). Noting that the trajectory vector $\boldsymbol{o}$ will always have a magnitude of $\alpha$, we can express the trajectory vector as

$$
\boldsymbol{o}=\alpha \hat{\boldsymbol{O}}
$$

and assuming that $\hat{\boldsymbol{o}}$ rotates relative to the body-fixed reference frame by some timevarying angular velocity $\boldsymbol{\omega}_{O B}$, the time derivative is easily solved as

$$
\dot{\boldsymbol{o}}=\alpha \boldsymbol{\omega}_{O I}^{\times} \hat{\boldsymbol{o}}
$$

where, of course, $\boldsymbol{\omega}_{O I}=\boldsymbol{\omega}_{O B}+\boldsymbol{\omega}_{B I}$. Some simple calculus results in

$$
\frac{\partial V_{a}}{\partial \boldsymbol{o}} \dot{\boldsymbol{o}}=\frac{d V_{a}}{d \theta}\left(\frac{\boldsymbol{\omega}_{O I}^{T} \hat{\boldsymbol{r}}^{\times} \hat{\boldsymbol{o}}}{\sin \theta}\right)
$$

From this result, let us try a function for $\boldsymbol{g}(\boldsymbol{r}, \dot{\boldsymbol{o}}, \cdot)$ defined by

$$
\boldsymbol{g}(\boldsymbol{r}, \dot{\boldsymbol{o}}, \cdot)=g(r, \theta) \boldsymbol{\omega}_{O I}^{\times} \hat{\boldsymbol{r}}
$$

where $g(r, \theta)$ is a non-negative function of distance $r$ and angle $\theta$, and is assumed to be Lipschitz continuous in both arguments. Let us mandate the condition that 
$g(\alpha, \theta)=\alpha$. Then, some calculus will show

$$
\frac{\partial V_{a}}{\partial \boldsymbol{r}} \boldsymbol{g}(\boldsymbol{r}, \dot{\boldsymbol{o}}, \cdot)=-\frac{d V_{a}}{d \theta}\left(\frac{g(r, \theta)}{r}\right)\left(\frac{\boldsymbol{\omega}_{O I}^{T} \hat{\boldsymbol{r}}^{\times} \hat{\boldsymbol{o}}}{\sin \theta}\right) .
$$

Evaluating Eq. (3.38) on $\mathbb{A}_{c}$ then gives

$$
\left.\frac{\partial V_{a}}{\partial \boldsymbol{r}} \boldsymbol{g}(\boldsymbol{r}, \dot{\boldsymbol{o}}, \cdot)\right|_{\boldsymbol{r} \in \mathbb{A}_{c}}=-\frac{d V_{a}}{d \theta}\left(\frac{\boldsymbol{\omega}_{O I}^{T} \hat{\boldsymbol{r}}^{\times} \hat{\boldsymbol{o}}}{\sin \theta}\right)=-\frac{\partial V_{a}}{\partial \boldsymbol{o}} \dot{\boldsymbol{o}}
$$

and therefore C5) can be easily replaced by mandating that $\boldsymbol{g}(\boldsymbol{r}, \dot{\boldsymbol{o}}, \cdot)$ has the structure given by Eq. (3.37) and that $g(\alpha, \theta)=\alpha$.

Next, let us focus on simplifying C2). Noting that $V_{c}$ is only a function of distance from $\mathbb{A}_{c}$, and that $\mathbb{A}_{c}$ is spherical, it is apparent that the contours of $V_{c}$ will also be spherical. Also noting that $B$ is centered in $\mathbb{A}_{c}$, it is noted that $V_{c}$ will no longer be dependent on the orientation of $B$. This simplifies $\mathrm{C} 2$ ) to

$$
\frac{d V_{c}}{d \boldsymbol{r}} \boldsymbol{\Gamma}_{a}(\boldsymbol{r}, \boldsymbol{o}, \cdot) \frac{\partial V_{a}^{T}}{\partial \boldsymbol{r}}=\frac{d V_{c}}{d \boldsymbol{r}} g(r, \theta) \boldsymbol{\omega}_{O I}^{\times} \hat{\boldsymbol{r}}
$$

Again, using the fact that $V_{c}$ is a function of distance from $\mathbb{A}_{c}$ only,

$$
\frac{d V_{c}}{d \boldsymbol{r}}=\left(\frac{d V_{c}}{d(r-\alpha)} \frac{d(r-\alpha)}{d r}\right) \frac{d r}{d \boldsymbol{r}}=c \hat{\boldsymbol{r}}^{T}
$$

where $c \in \mathbb{R}$ is some number. Therefore, Eq. (3.40) further simplies to

$$
\hat{\boldsymbol{r}}^{T} \boldsymbol{\Gamma}_{a}(\boldsymbol{r}, \boldsymbol{o}, \cdot) \frac{\partial V_{a}^{T}}{\partial \boldsymbol{r}}=0
$$

Therefore, with the simplifying assumptions made in this subsection, C2) can be replaced by the condition that $\boldsymbol{a}(\boldsymbol{r}, \boldsymbol{o}, \cdot)=-\boldsymbol{\Gamma}_{a}(\boldsymbol{r}, \boldsymbol{o}, \cdot) \frac{\partial V_{a}{ }^{T}}{\partial \boldsymbol{r}}$ is perpendicular to $\hat{\boldsymbol{r}}$.

\subsubsection{Robustness of the Alignment Field Stability to Small Contraction Errors}

For the stability analysis of Sec. 3.2.2, it should be noted that asymptotic stability of the point $\theta=0$ is only (in general) achieved if $\boldsymbol{r}$ exactly converges onto the boundary 
$\mathbb{A}_{c}$. Of course, in any practical setting there will be a small convergence error, and so robustness of this claim should be studied. For this analysis, it is assumed that the simplifying assumptions of Sec. 3.2.3 apply, and therefore $\mathbb{A}_{c}$ is spherical, the origin of $B$ is centered in $\mathbb{A}$, and $V_{c}$ is only a function of $|r-\alpha|$. Constructing a perturbation vector

$$
\Delta \boldsymbol{r}=\Delta \boldsymbol{r}_{n}+\Delta \boldsymbol{r}_{t}
$$

such that, if a plane tangent to $\mathbb{A}_{c}$ is constructed at the point $\boldsymbol{r}$, then $\Delta \boldsymbol{r}_{n}$ is normal to the plane, and $\Delta \boldsymbol{r}_{t}$ is a step within the plane. Given a sufficiently small step in the tangent direction $\Delta \boldsymbol{r}_{t}$, the position vector $\boldsymbol{r}$ approximately remains within $\mathbb{A}_{c}$, and it is primarily perturbations in the normal direction $\Delta \boldsymbol{r}_{n}$ which are driven to $\mathbf{0}$ by Theorem 3. Let us label an equilibrium point (i.e., either $(r, \theta)=(\alpha, 0)$ or $(r, \theta)=(\alpha, \pi))$ by $\boldsymbol{r}^{*}$. Using a Taylor expansion, the term $\dot{V}_{a}\left(\boldsymbol{r}^{*}+\Delta \boldsymbol{r}, \boldsymbol{o}\right)$ can be approximated as

$$
\dot{V}_{a}\left(\boldsymbol{r}^{*}+\Delta \boldsymbol{r}, \boldsymbol{o}\right) \approx \dot{V}_{a}\left(\boldsymbol{r}^{*}, \boldsymbol{o}\right)+\frac{\partial \dot{V}_{a}}{\partial \boldsymbol{r}}\left(\boldsymbol{r}^{*}, \boldsymbol{o}\right) \Delta \boldsymbol{r}=\frac{\partial \dot{V}_{a}}{\partial \boldsymbol{r}}\left(\boldsymbol{r}^{*}, \boldsymbol{o}\right) \Delta \boldsymbol{r}
$$

Then, using Eq. (3.28) the general Jacobian $\frac{\partial \dot{V}_{a}}{\partial \boldsymbol{r}}(\boldsymbol{r}, \boldsymbol{o})$ is found to be

$$
\frac{\partial \dot{V}_{a}}{\partial \boldsymbol{r}}(\boldsymbol{r}, \boldsymbol{o})=(\boldsymbol{c}+\boldsymbol{a})^{T} \frac{\partial^{2} V_{a}}{\partial \boldsymbol{r}^{2}}+\frac{\partial V_{a}}{\partial \boldsymbol{r}}\left(\frac{\partial \boldsymbol{c}}{\partial \boldsymbol{r}}+\frac{\partial \boldsymbol{a}}{\partial \boldsymbol{r}}\right)+\frac{\partial}{\partial \boldsymbol{r}}\left(\frac{\partial V_{a}}{\partial \boldsymbol{r}} \boldsymbol{g}+\frac{\partial V_{a}}{\partial \boldsymbol{o}} \dot{\boldsymbol{o}}\right)
$$

where arguments have been dropped for notational simplicity. Defining the error function

$$
\psi(\boldsymbol{r}, \dot{\boldsymbol{o}}, \cdot)=\frac{\partial V_{a}}{\partial \boldsymbol{r}} \boldsymbol{g}+\frac{\partial V_{a}}{\partial \boldsymbol{o}} \dot{\boldsymbol{o}}
$$

the general Jacobian can then be written

$$
\frac{\partial \dot{V}_{a}}{\partial \boldsymbol{r}}(\boldsymbol{r}, \boldsymbol{o})=(\boldsymbol{c}+\boldsymbol{a})^{T} \frac{\partial^{2} V_{a}}{\partial \boldsymbol{r}^{2}}+\frac{\partial V_{a}}{\partial \boldsymbol{r}}\left(\frac{\partial \boldsymbol{c}}{\partial \boldsymbol{r}}+\frac{\partial \boldsymbol{a}}{\partial \boldsymbol{r}}\right)+\frac{\partial \psi}{\partial \boldsymbol{r}}
$$


Note that on an equilibrium point $\boldsymbol{r}^{*}$, the condition holds that $\boldsymbol{c}=\boldsymbol{a}=\mathbf{0}$, and therefore

$$
\frac{\partial \dot{V}_{a}}{\partial \boldsymbol{r}}\left(\boldsymbol{r}^{*}, \boldsymbol{o}\right)=\frac{\partial V_{a}}{\partial \boldsymbol{r}}\left(\boldsymbol{r}^{*}, \boldsymbol{o}, \cdot\right)\left(\frac{\partial \boldsymbol{c}}{\partial \boldsymbol{r}}\left(\boldsymbol{r}^{*}, \cdot\right)+\frac{\partial \boldsymbol{a}}{\partial \boldsymbol{r}}\left(\boldsymbol{r}^{*}, \boldsymbol{o}, \cdot\right)\right)+\frac{\partial \psi}{\partial \boldsymbol{r}}\left(\boldsymbol{r}^{*}, \boldsymbol{o}, \cdot\right)
$$

substituting Eq. (3.48) into Eq. (3.44) leads to

$$
\dot{V}_{a}\left(\boldsymbol{r}^{*}+\Delta \boldsymbol{r}, \boldsymbol{o}\right) \approx \frac{\partial V_{a}}{\partial \boldsymbol{r}}\left(\boldsymbol{r}^{*}, \boldsymbol{o}, \cdot\right)\left(\frac{\partial \boldsymbol{c}}{\partial \boldsymbol{r}}\left(\boldsymbol{r}^{*}, \cdot\right) \Delta \boldsymbol{r}+\frac{\partial \boldsymbol{a}}{\partial \boldsymbol{r}}\left(\boldsymbol{r}^{*}, \boldsymbol{o}, \cdot\right) \Delta \boldsymbol{r}\right)+\frac{\partial \psi}{\partial \boldsymbol{r}}\left(\boldsymbol{r}^{*}, \boldsymbol{o}, \cdot\right) \Delta \boldsymbol{r}
$$

Now, consider the product $\frac{\partial \boldsymbol{a}}{\partial \boldsymbol{r}}\left(\boldsymbol{r}^{*}, \boldsymbol{o}, \cdot\right) \Delta \boldsymbol{r}$. Assuming $\boldsymbol{a}(\boldsymbol{r}, \boldsymbol{o}, \cdot)$ has been constructed such that it equals $\mathbf{0}$ along the line of $\boldsymbol{r}$ where $\theta=0$ or $\theta=\pi$, the matrix $\frac{\partial \boldsymbol{a}}{\partial \boldsymbol{r}}\left(\boldsymbol{r}^{*}, \boldsymbol{o}, \cdot\right)$ has a null-space defined by the line $\theta=0$ or $\theta=\pi$. That is,

$$
\frac{\partial \boldsymbol{a}}{\partial \boldsymbol{r}}\left(\boldsymbol{r}^{*}, \boldsymbol{o}, \cdot\right) \Delta \boldsymbol{r}=\frac{\partial \boldsymbol{a}}{\partial \boldsymbol{r}}\left(\boldsymbol{r}^{*}, \boldsymbol{o}, \cdot\right)\left(\Delta \boldsymbol{r}_{t}+\Delta \boldsymbol{r}_{n}\right) \approx \frac{\partial \boldsymbol{a}}{\partial \boldsymbol{r}}\left(\boldsymbol{r}^{*}, \boldsymbol{o}, \cdot\right) \Delta \boldsymbol{r}_{t}
$$

Carrying out a similar procedure for $\frac{\partial \boldsymbol{c}}{\partial \boldsymbol{r}}\left(\boldsymbol{r}^{*}, \cdot\right) \Delta \boldsymbol{r}$ and $\frac{\partial \psi}{\partial \boldsymbol{r}}\left(\boldsymbol{r}^{*}, \boldsymbol{o}, \cdot\right) \Delta \boldsymbol{r}$ and substituting each back into Eq. (3.49) results in

$$
\dot{V}_{a}\left(\boldsymbol{r}^{*}+\Delta \boldsymbol{r}, \boldsymbol{o}\right) \approx \frac{\partial V_{a}}{\partial \boldsymbol{r}}\left(\boldsymbol{r}^{*}, \boldsymbol{o}, \cdot\right)\left(\frac{\partial \boldsymbol{c}}{\partial \boldsymbol{r}}\left(\boldsymbol{r}^{*}, \cdot\right) \Delta \boldsymbol{r}_{n}+\frac{\partial \boldsymbol{a}}{\partial \boldsymbol{r}}\left(\boldsymbol{r}^{*}, \boldsymbol{o}, \cdot\right) \Delta \boldsymbol{r}_{t}\right)+\frac{\partial \psi}{\partial \boldsymbol{r}}\left(\boldsymbol{r}^{*}, \boldsymbol{o}, \cdot\right) \Delta \boldsymbol{r}_{n}
$$

or, grouping perturbation terms $\Delta \boldsymbol{r}_{n}$ and $\Delta \boldsymbol{r}_{t}$

$\dot{V}_{a}\left(\boldsymbol{r}^{*}+\Delta \boldsymbol{r}, \boldsymbol{o}\right) \approx \frac{\partial V_{a}}{\partial \boldsymbol{r}}\left(\boldsymbol{r}^{*}, \boldsymbol{o}, \cdot\right) \frac{\partial \boldsymbol{a}}{\partial \boldsymbol{r}}\left(\boldsymbol{r}^{*}, \boldsymbol{o}, \cdot\right) \Delta \boldsymbol{r}_{t}+\left(\frac{\partial V_{a}}{\partial \boldsymbol{r}}\left(\boldsymbol{r}^{*}, \boldsymbol{o}, \cdot\right) \frac{\partial \boldsymbol{c}}{\partial \boldsymbol{r}}\left(\boldsymbol{r}^{*}, \cdot\right)+\frac{\partial \psi}{\partial \boldsymbol{r}}\left(\boldsymbol{r}^{*}, \boldsymbol{o}, \cdot\right)\right) \Delta \boldsymbol{r}_{n}$

where the term $\frac{\partial V_{a}}{\partial \boldsymbol{r}}\left(\boldsymbol{r}^{*}, \boldsymbol{o}, \cdot\right) \frac{\partial \boldsymbol{a}}{\partial \boldsymbol{r}}\left(\boldsymbol{r}^{*}, \boldsymbol{o}, \cdot\right) \Delta \boldsymbol{r}_{t}$ is a Taylor approximation to the stabilizing portion of $\dot{V}_{a}$ (and will therefore be strictly negative when perturbed off of the equilibrium points), and the second term is a Taylor approximation of the disturbance $\delta(\boldsymbol{r})$ from Theorem 4 . Solving for a (conservative) region where $\dot{V}_{a} \geq 0$ occurs yields

$$
\left\|\Delta \boldsymbol{r}_{t}\right\| \leq\left(\frac{\left\|\frac{\partial V_{a}}{\partial \boldsymbol{r}}\left(\boldsymbol{r}^{*}, \boldsymbol{o}, \cdot\right) \frac{\partial \boldsymbol{c}}{\partial \boldsymbol{r}}\left(\boldsymbol{r}^{*}, \cdot\right)+\frac{\partial \phi}{\partial \boldsymbol{r}}\left(\boldsymbol{r}^{*}, \boldsymbol{o}, \cdot\right)\right\|}{\left\|\frac{\partial V_{a}}{\partial \boldsymbol{r}}\left(\boldsymbol{r}^{*}, \boldsymbol{o}, \cdot\right) \frac{\partial \boldsymbol{a}}{\partial \boldsymbol{r}}\left(\boldsymbol{r}^{*}, \boldsymbol{o}, \cdot\right)\right\|}\right)\left\|\Delta \boldsymbol{r}_{n}\right\|
$$


or, solving for the "thickness" of the regions surrounding $\theta=0$ or $\theta=\pi$ where $\dot{V}_{a} \geq 0$ could possibly occur, given by $\Delta \theta=\frac{\left\|\Delta \boldsymbol{r}_{t}\right\|}{\alpha}$ yields

$$
\Delta \theta \leq\left(\frac{\left\|\frac{\partial V_{a}}{\partial \boldsymbol{r}}\left(\boldsymbol{r}^{*}, \boldsymbol{o}, \cdot\right) \frac{\partial \boldsymbol{c}}{\partial \boldsymbol{r}}\left(\boldsymbol{r}^{*}, \cdot\right)+\frac{\partial \psi}{\partial \boldsymbol{r}}\left(\boldsymbol{r}^{*}, \boldsymbol{o}, \cdot\right)\right\|}{\alpha\left\|\frac{\partial V_{a}}{\partial \boldsymbol{r}}\left(\boldsymbol{r}^{*}, \boldsymbol{o}, \cdot\right) \frac{\partial \boldsymbol{a}}{\partial \boldsymbol{r}}\left(\boldsymbol{r}^{*}, \boldsymbol{o}, \cdot\right)\right\|}\right)\left\|\Delta \boldsymbol{r}_{n}\right\|
$$

Equation (3.54) therefore gives the intuitive result that for some small convergence error $\left\|\Delta \boldsymbol{r}_{n}\right\|$, the resulting size of the unstable regions is dependent on the ratio of how quickly the destabilizing disturbance grows compared to how quickly the stabilizing guidance grows. Importantly, since the designer completely controls $V_{a}$, $\boldsymbol{a}$, and $\boldsymbol{c}$, and can influence $\psi$ by the design of $\boldsymbol{g}$, the ratio $\frac{\Delta \theta}{\left\|\Delta \boldsymbol{r}_{n}\right\|}$ can be made arbitrarily low. The trade off is that by making this ratio too low, the denominator $\left\|\frac{\partial V_{a}}{\partial \boldsymbol{r}}\left(\boldsymbol{r}^{*}, \boldsymbol{o}, \cdot\right) \frac{\partial \boldsymbol{a}}{\partial \boldsymbol{r}}\left(\boldsymbol{r}^{*}, \boldsymbol{o}, \cdot\right)\right\|$ is required to be relatively large, which results in poor acceleration characteristics near $\theta=0$ or $\theta=\pi($ i.e., $\boldsymbol{a}(\boldsymbol{r}, \boldsymbol{o}, \cdot)$ becomes high-gain feedback on $\theta$, amplifying noise).

From a design perspective, Eq. (3.54) should be used to evaluate the robustness of alignment stability to small errors in convergence after specific functions $V_{a}(\theta)$, $\boldsymbol{c}(\boldsymbol{r}, \cdot), \boldsymbol{a}(\boldsymbol{r}, \theta, \cdot)$ and $\boldsymbol{g}(\boldsymbol{r}, \dot{\boldsymbol{o}}, \cdot)$ have been selected. As a final remark, note that if $\boldsymbol{c}(\boldsymbol{r}, \cdot)$ is selected such that it is along the vector $\hat{\boldsymbol{r}}$, then Eq. (3.54) simplifies to

$$
\Delta \theta \leq\left(\frac{\left\|\frac{\partial \psi}{\partial \boldsymbol{r}}\left(\boldsymbol{r}^{*}, \boldsymbol{o}, \cdot\right)\right\|}{\alpha\left\|\frac{\partial V_{a}}{\partial \boldsymbol{r}}\left(\boldsymbol{r}^{*}, \boldsymbol{o}, \cdot\right) \frac{\partial \boldsymbol{a}}{\partial \boldsymbol{r}}\left(\boldsymbol{r}^{*}, \boldsymbol{o}, \cdot\right)\right\|}\right)\left\|\Delta \boldsymbol{r}_{n}\right\|
$$

\subsection{Acceleration Constraints}

In this section, the acceleration properties of a simplified CLVF will be studied. Beyond the assumptions made in Sec. 3.2.3, it is also assumed that the contraction field points in the steepest descent direction of $V_{c}$, and the alignment field points in the steepest descent direction of $V_{a}$. Mathematically, this corresponds to the matrices $\boldsymbol{\Gamma}_{a}(\boldsymbol{r}, \boldsymbol{o}, \cdot)$ and $\boldsymbol{\Gamma}_{c}(\boldsymbol{r}, \cdot)$ being multiples of the identity matrix.

While these additional simplifying assumptions may seem limiting, it should be 
noted that all of these assumptions are used in the overwhelming majority of published work regarding LVFs. For example, the $\boldsymbol{\Gamma}$ matrix is considered implicitly to be a multiple of the identity matrix in $[14,15,20-23]$ as the contraction component of velocity is is simply defined as the negative gradient direction. In [17], the general positive definite $\boldsymbol{\Gamma}$ is presented, but every example within the paper chooses a $\boldsymbol{\Gamma}$ which is a multiple of identity. In [16], the proposed altered LVF explicitly includes a scaled identity matrix for $\boldsymbol{\Gamma}$. The only work where an identity matrix is not explicitly used for the the $\boldsymbol{\Gamma}$ matrix is [18], where the entire LVF is selected by machine learning. The circular attractor and centered target assumptions are equally broadly applied (for cases where the attractor consists of more than a single point). Therefore, the assumptions utilized in this Section are quite practical, as evidenced by their broad application in the existing literature.

In Sec. 3.3.1 the assumptions of $\boldsymbol{\Gamma}$ matrices which are scaled identity matrices is used to express the CLVF in a simplified form. In Sec. 3.3.2, conditions on this simplified form are derived to guarantee that an upper-bound on the required acceleration for perfect tracking exists. A more compact form of the resulting feedforward equations (useful for finding an actual value of the acceleration bound) is then presented and analyzed in Sec. 3.3.3. Although not the focus of this work, Sec. 3.3.4 briefly analyzes the control law of Eq. (2.7) proposed by Lawrence et. al [17] to highlight that in most cases, the CLVF can still make use of this controller, however some thruster saturation may result while the controlled vehicle initially converges to the CLVF guidance.

\subsubsection{Simplified Form of CLVF}

Assuming that $\boldsymbol{\Gamma}_{c}$ and $\boldsymbol{\Gamma}_{a}$ are scaled identity matrices, let us begin by rewriting these matrices in the form

$$
\begin{gathered}
\boldsymbol{\Gamma}_{c}(\boldsymbol{r}, \cdot)=\frac{s_{c}(r, \theta)}{\left\|d V_{c} / d \boldsymbol{r}\right\|} \boldsymbol{I} \\
\boldsymbol{\Gamma}_{a}(\boldsymbol{r}, \hat{\boldsymbol{o}}, \cdot)=\frac{s_{a}(r, \theta)}{\left\|\partial V_{a} / \partial \boldsymbol{r}\right\|} \boldsymbol{I}
\end{gathered}
$$


where $s_{c}(r, \theta)$ and $s_{a}(r, \theta)$ are yet-unselected speed functions which are globally Lipshcitz in both $r$ and $\theta$ (otherwise globally constraining accelerations would become problematic), and must meet the conditions C3) and C6) for $\boldsymbol{\Gamma}_{c}$ and $\boldsymbol{\Gamma}_{a}$. That is,

$$
s_{c}(\alpha, \theta)=0
$$

and is otherwise strictly positive, and $s_{a}(r, \theta)$ may be designed such that

$$
\lim _{\theta \rightarrow 0} s_{a}(r, \theta)=\lim _{\theta \rightarrow \pi} s_{a}(r, \theta)=0
$$

but is strictly positive otherwise. Assuming the structure for $\boldsymbol{g}(\boldsymbol{r}, \dot{\boldsymbol{o}}, \cdot)$ from Sec. 3.2.3 is used, the CLVF can now be written in the form

$$
\boldsymbol{h}\left(\boldsymbol{r}, \boldsymbol{\omega}_{O I}, \hat{\boldsymbol{o}}, \dot{\boldsymbol{d}}\right)=s_{c}(r, \theta) \hat{\boldsymbol{c}}(\boldsymbol{r})+s_{a}(r, \theta) \hat{\boldsymbol{a}}(\hat{\boldsymbol{r}}, \hat{\boldsymbol{o}})+g(r, \theta) \boldsymbol{\omega}_{O I}^{\times} \hat{\boldsymbol{r}}+\dot{\boldsymbol{d}}
$$

where

$$
\begin{gathered}
\hat{\boldsymbol{c}}=-\frac{\left(\partial V_{c} / \partial \boldsymbol{r}\right)^{T}}{\left\|\partial V_{c} / \partial \boldsymbol{r}\right\|} \\
\hat{\boldsymbol{a}}=-\frac{\left(\partial V_{a} / \partial \boldsymbol{r}\right)^{T}}{\left\|\partial V_{a} / \partial \boldsymbol{r}\right\|}
\end{gathered}
$$

Note that by using the assumption that $V_{c}$ is a function of the distance from the attractor only, and setting $\hat{\boldsymbol{c}}$ to the null vector $\mathbf{0}$ when $\|\boldsymbol{r}\|=\alpha$ to avoid singularities in the solution, $\hat{\boldsymbol{c}}$ equates to

$$
\hat{\boldsymbol{c}}= \begin{cases}-\hat{\boldsymbol{r}}, & \|\boldsymbol{r}\|>\alpha \\ \hat{\boldsymbol{r}}, & \|\boldsymbol{r}\|<\alpha \\ \mathbf{0}, & \text { otherwise }\end{cases}
$$

Next, using the term $\frac{\partial \theta}{\partial \boldsymbol{r}}$ from Eq. (3.32), we note that

$$
\left\|\frac{\partial \theta}{\partial \boldsymbol{r}}\right\|=\left(\frac{\partial \theta}{\partial \boldsymbol{r}} \frac{\partial \theta^{T}}{\partial \boldsymbol{r}}\right)^{1 / 2}=\frac{\left(1-\cos ^{2} \theta\right)^{1 / 2}}{r \sin \theta}=\frac{1}{r}
$$


such that $\hat{\boldsymbol{a}}$ can be defined as

$$
\hat{\boldsymbol{a}}=-r \frac{\partial \theta^{T}}{\partial \boldsymbol{r}}
$$

to have unity magnitude. To avoid singularities at $\theta=0$ and $\theta=\pi, \hat{\boldsymbol{a}}$ can be therefore be computed as

$$
\hat{\boldsymbol{a}}= \begin{cases}\frac{\hat{\boldsymbol{o}}-\hat{\boldsymbol{r}} \cos \theta}{\sin \theta}, & 0<\theta<\pi, \\ \mathbf{0}, & \text { otherwise. }\end{cases}
$$

which also leads to the following useful relationship between $\hat{\boldsymbol{o}}, \hat{\boldsymbol{a}}$ and $\hat{\boldsymbol{r}}$

$$
\hat{\boldsymbol{o}}=\hat{\boldsymbol{r}} \cos \theta+\hat{\boldsymbol{a}} \sin \theta
$$

Lastly, the product $s_{c}(r, \theta) \hat{\boldsymbol{c}}$ can be rewritten as

$$
s_{c}(r, \theta) \hat{\boldsymbol{c}}=v_{c}(r, \theta) \hat{\boldsymbol{r}}
$$

where $v_{c}$ is referred to as the contraction velocity function, as it encodes the desired direction along the position vector $\boldsymbol{r}$. That is

$$
v_{c}(r, \theta)= \begin{cases}s_{c}(r, \theta), & r<\alpha \\ -s_{c}(r, \theta), & \text { otherwise }\end{cases}
$$

The main benefit of expressing the contraction velocity in this way is we can then define a third vector unit vector

$$
\hat{\boldsymbol{e}}=\hat{\boldsymbol{r}}^{\times} \hat{\boldsymbol{a}}
$$

which will be useful in the acceleration analysis. If instead the vector $\hat{\boldsymbol{c}}$ were used to define $\hat{\boldsymbol{e}}$ with a cross-product, then the direction of $\hat{\boldsymbol{e}}$ would change whenever the sphere $r=\alpha$ was crossed. With this new unit vector $\hat{\boldsymbol{e}}$ being defined, it is useful to note that for every $\boldsymbol{r}$ except the line where $\theta=0$ or $\theta=\pi$, the following identity holds

$$
\hat{\boldsymbol{r}} \hat{\boldsymbol{r}}^{T}+\hat{\boldsymbol{a}} \hat{\boldsymbol{a}}^{T}+\hat{\boldsymbol{e}} \hat{\boldsymbol{e}}^{T}=\boldsymbol{I}
$$

The final special form of the CLVF to be used for analysis in the remainder of this 
work is then

$$
\boldsymbol{h}\left(\boldsymbol{r}, \hat{\boldsymbol{o}}, \boldsymbol{\omega}_{O I}, \dot{\boldsymbol{d}}\right)=v_{c}(r, \theta) \hat{\boldsymbol{r}}+s_{a}(r, \theta) \hat{\boldsymbol{a}}+g(r, \theta) \boldsymbol{\omega}_{O I}^{\times} \hat{\boldsymbol{r}}+\dot{\boldsymbol{d}}
$$

and the stability constraints C1)-C6) can be translated to the following smaller set of conditions on $v_{c}(r, \theta), s_{a}(r, \theta)$ and $g(r, \theta)$

D1) $\operatorname{sgn}\left(v_{c}(r, \theta)\right)=\operatorname{sgn}(\alpha-r)$, and $v_{c}(\alpha, \theta)=0$

D2) $g(r, \theta) \geq 0$, and $g(\alpha, \theta)=\alpha$

D3) It is allowed that $\lim _{\theta \rightarrow 0} s_{a}(r, \theta)=\lim _{\theta \rightarrow \pi} s_{a}(r, \theta)=0$, but $s_{a}(r, \theta)>0$ otherwise.

\subsubsection{Upper-Boundedness of the Required Tracking Acceleration}

In this subsection, a sufficient set of conditions are derived on the velocity and distance functions $v_{c}(r, \theta), s_{a}(r, \theta)$ and $g(r, \theta)$ which guarantee that the acceleration to perfectly track the CLVF is bounded everywhere.

To begin, because only Lipschitz continuity (rather than continuous differentiablity) is assumed, let us analyze a discrete change in the CLVF, assumed to have occurred over some finite period of time $\Delta t$.

$$
\Delta h=\Delta c+\Delta a+\Delta g+\Delta \dot{d}
$$

where, for some variable $\zeta$ of any given dimension and a time index $k$, the discrete delta operator is defined as

$$
\Delta \zeta=\zeta(k+1)-\zeta(k)
$$

Of course, if the acceleration magnitude is constrained by $u_{\max } \in \mathbb{R}>0$, then by definition the CLVF is Lipschitz continuous in time such that

$$
\|\Delta \boldsymbol{h}\| \leq u_{\max }\|\Delta t\|
$$


This implies that there is a "weak" derivative of $\boldsymbol{h}$; that is, $\boldsymbol{h}$ can be expressed as a bounded piecewise-continuous function made up of $K \in \mathbb{Z}$ continuous functions on their own time intervals $\left(t_{i-1}, t_{i}\right)$, and that the discrete difference $\Delta \boldsymbol{h}$ can alternatively be expressed [27]

$$
\Delta \boldsymbol{h}=\sum_{i=1}^{K} \int_{t_{i-1}}^{t_{i}} \dot{\boldsymbol{h}} d t
$$

where, for the remainder of this work bounds will be dropped, with the understanding that

$$
\Delta \boldsymbol{h}=\sum \int \dot{\boldsymbol{h}} d t
$$

represents the integral of a bounded piecewise-continuous function $(\dot{\boldsymbol{h}}$, in the example above) to form a bounded discrete step. The simplest way to ensure that $\boldsymbol{h}$ is Lipschitz continuous in time is to mandate that each of the sub-functions $\boldsymbol{c}, \boldsymbol{a}, \boldsymbol{g}$ and $\dot{\boldsymbol{d}}$ are themselves Lipschitz continuous in time. To begin, let us express the first term in Eq. (3.73) as

$$
\Delta \boldsymbol{c}=\Delta\left(v_{c}(r, \theta) \hat{\boldsymbol{r}}\right)=\sum \int\left(\dot{v}_{c}(r, \theta) \hat{\boldsymbol{r}}+v_{c}(r, \theta) \dot{\hat{\boldsymbol{r}}}\right) d t
$$

The time-derivative $\dot{v}_{c}$ can be expressed (on the intervals where it is continuous) as

$$
\dot{v}_{c}(r, \theta)=\frac{\partial v_{c}}{\partial r} \dot{r}+\frac{\partial v_{c}}{\partial \theta} \dot{\theta}
$$

Because $v_{c}(r, \theta)$ is already assumed to be Lipschitz continuous in both $r$ and $\theta, \dot{v}_{c}(r, \theta)$ is bounded if $\dot{r}$ and $\dot{\theta}$ are bounded. When perfect tracking of the CLVF is assumed, the following expressions on the intervals of continuity for $\dot{r}$ and $\dot{\theta}$ can be derived

$$
\begin{aligned}
\left.\dot{r}\right|_{\dot{\boldsymbol{r}}=\boldsymbol{h}} & =\left.\frac{d r}{d t}\right|_{\dot{\boldsymbol{r}}=\boldsymbol{h}} \\
& =\frac{d r}{d \boldsymbol{r}}(\boldsymbol{h}-\dot{\boldsymbol{d}}) \\
& =\hat{\boldsymbol{r}}^{T}\left(v_{c}(r, \theta) \hat{\boldsymbol{r}}+s_{a}(r, \theta) \hat{\boldsymbol{a}}+g(r, \theta) \boldsymbol{\omega}_{O I}^{\times} \hat{\boldsymbol{r}}\right) \\
& =v_{c}(r, \theta)
\end{aligned}
$$


so $\left.\dot{r}\right|_{\dot{r}=\boldsymbol{h}}$ is bounded if $v_{c}(r, \theta)$ is bounded. Next, for $\dot{\theta}$

$$
\begin{aligned}
\left.\dot{\theta}\right|_{\dot{\boldsymbol{r}}=\boldsymbol{h}} & =\left.\left(\frac{\partial \theta}{\partial \hat{\boldsymbol{r}}} \frac{d \hat{\boldsymbol{r}}}{d \boldsymbol{r}} \dot{\boldsymbol{r}}+\frac{\partial \theta}{\partial \hat{\boldsymbol{o}}}\right)\right|_{\dot{\boldsymbol{r}}=\boldsymbol{h}} \\
& =\left(\frac{-\hat{\boldsymbol{o}}^{T}}{\sin \theta}\right)\left(\frac{\boldsymbol{I}-\hat{\boldsymbol{r}} \hat{\boldsymbol{r}}^{T}}{r}\right)\left(v_{c}(r, \theta) \hat{\boldsymbol{r}}+s_{a}(r, \theta) \hat{\boldsymbol{a}}+g(r, \theta) \boldsymbol{\omega}_{O I}^{\times} \hat{\boldsymbol{r}}\right)+\left(\frac{-\hat{\boldsymbol{r}}^{T}}{\sin \theta}\right)\left(\boldsymbol{\omega}_{O I}^{\times} \hat{\boldsymbol{o}}\right) \\
& =-\frac{s_{a}(r, \theta)}{r}+\left(1-\frac{g(r, \theta)}{r}\right) \boldsymbol{\omega}_{O I}^{T} \hat{\boldsymbol{e}}
\end{aligned}
$$

where Eqs. (3.67), (3.70) and (3.71) have been used in the final step. Note, the fact that Eq. (3.71) does not apply at $\theta=0$ or $\theta=\pi$ is not of concern, as crossing one of these points would represent discontinuities in the derivative, and therefore these points are excluded from the integrals of Eq. (3.79). Obviously, it is necessary that $\boldsymbol{\omega}_{O I}$ is bounded, otherwise it is impossible to track. Therefore $\left.\dot{\theta}\right|_{\dot{\boldsymbol{r}}=\boldsymbol{h}}$ is bounded if $\frac{s_{a}(r, \theta)}{r}$ and $\frac{g(r, \theta)}{r}$ are bounded. That is, if $r \rightarrow 0$, then it is required that $s_{a}(r, \theta) \rightarrow 0$ and $g(r, \theta) \rightarrow 0$ at least as fast as $r$.

Because $v_{c}(r, \theta)$ is bounded, proving that $\left.\dot{\hat{\boldsymbol{r}}}\right|_{\dot{\boldsymbol{r}}=\boldsymbol{h}}$ is bounded will prove that Eq. (3.78) is the integral of a piecewise continuous bounded function, and therefore $\boldsymbol{c}(\boldsymbol{r}, \hat{\boldsymbol{o}})=v_{c}(r, \theta) \hat{\boldsymbol{r}}$ is Lipschitz continuous in time. Taking the derivative of $\hat{\boldsymbol{r}}$ yields

$$
\begin{aligned}
\left.\dot{\hat{\boldsymbol{r}}}\right|_{\dot{\boldsymbol{r}}=\boldsymbol{h}} & =\left.\frac{d \hat{\boldsymbol{r}}}{d \boldsymbol{r}}\right|_{\dot{\boldsymbol{r}}=\boldsymbol{h}} \\
& =\left(\frac{\boldsymbol{I}-\hat{\boldsymbol{r}} \hat{\boldsymbol{r}}^{T}}{r}\right)\left(v_{c}(r, \theta) \hat{\boldsymbol{r}}+s_{a}(r, \theta) \hat{\boldsymbol{a}}+g(r, \theta) \boldsymbol{\omega}_{O I}^{\times} \hat{\boldsymbol{r}}\right) \\
& =\frac{s_{a}(r, \theta) \hat{\boldsymbol{a}}+g(r, \theta) \boldsymbol{\omega}_{O I}^{\times} \hat{\boldsymbol{r}}}{r}
\end{aligned}
$$

which is already bounded by the conditions that $\boldsymbol{\omega}_{O I}, \frac{s_{a}(r, \theta)}{r}$, and $\frac{g(r, \theta)}{r}$ are bounded.

Next, let us examine the conditions required for $\boldsymbol{a}(\boldsymbol{r}, \hat{\boldsymbol{o}})=s_{a}(r, \theta) \hat{\boldsymbol{a}}$ to be Lipschitz time-continuous. Analogous to the previous developments, we begin with

$$
\Delta \boldsymbol{a}=\Delta\left(s_{a}(r, \theta) \hat{\boldsymbol{a}}\right)=\sum \int\left(\dot{s}_{a}(r, \theta) \hat{\boldsymbol{a}}+s_{a}(r, \theta) \dot{\hat{\boldsymbol{a}}}\right) d t
$$


Noting that $\left.\dot{r}\right|_{\dot{\boldsymbol{r}}=\boldsymbol{h}}$ and $\left.\dot{\theta}\right|_{\dot{\boldsymbol{r}}=\boldsymbol{h}}$ are already bounded and that $s_{a}(r, \theta)$ is assumed to be Lipschitz in $r$ and $\theta$, it immediately follows that $\left.\dot{s}_{a}(r, \theta)\right|_{\dot{r}=\boldsymbol{h}}$ is bounded (and indeed, so is $\left.\left.\dot{g}(r, \theta)\right|_{\dot{\boldsymbol{r}}=\boldsymbol{h}}\right)$. Therefore it is sufficient to show that the product $\left.s_{a}(r, \theta) \dot{\hat{\boldsymbol{a}}}\right|_{\dot{\boldsymbol{r}}=\boldsymbol{h}}$ is bounded. To show this, let us derive the time-derivative of $\hat{\boldsymbol{a}}$

$$
\dot{\hat{\boldsymbol{a}}}=\frac{\partial \hat{\boldsymbol{a}}}{\partial \hat{\boldsymbol{r}}} \dot{\hat{\boldsymbol{r}}}+\frac{\partial \hat{\boldsymbol{a}}}{\partial \hat{\boldsymbol{o}}} \dot{\hat{\boldsymbol{o}}}
$$

where

$$
\frac{\partial \hat{\boldsymbol{a}}}{\partial \hat{\boldsymbol{r}}}=\frac{d \hat{\boldsymbol{a}}}{d\left(\hat{\boldsymbol{o}} \hat{\boldsymbol{r}}^{T} \hat{\boldsymbol{r}}-\hat{\boldsymbol{r}} \hat{\boldsymbol{r}}^{T} \hat{\boldsymbol{o}}\right)} \frac{\partial\left(\hat{\boldsymbol{o}} \hat{\boldsymbol{r}}^{T} \hat{\boldsymbol{r}}-\hat{\boldsymbol{r}} \hat{\boldsymbol{r}}^{T} \hat{\boldsymbol{o}}\right)}{\partial \hat{\boldsymbol{r}}}=\left(\frac{\boldsymbol{I}-\hat{\boldsymbol{a}} \hat{\boldsymbol{a}}^{T}}{\sin \theta}\right)\left(2 \hat{\boldsymbol{o}} \hat{\boldsymbol{r}}^{T}-\hat{\boldsymbol{r}} \hat{\boldsymbol{o}}^{T}-\hat{\boldsymbol{r}}^{T} \hat{\boldsymbol{o}} \boldsymbol{I}\right)
$$

and similarly

$$
\frac{\partial \hat{\boldsymbol{a}}}{\partial \hat{\boldsymbol{o}}}=\left(\frac{\boldsymbol{I}-\hat{\boldsymbol{a}} \hat{\boldsymbol{a}}^{T}}{\sin \theta}\right)\left(\boldsymbol{I}-\hat{\boldsymbol{r}} \hat{\boldsymbol{r}}^{T}\right)
$$

Substituting Eq. (3.89) into Eq. (3.91) and simplifying yields

$$
\left.\dot{\hat{\boldsymbol{a}}}\right|_{\dot{\boldsymbol{r}}=\boldsymbol{h}}=\frac{-s_{a}(r, \theta)-g(r, \theta) \boldsymbol{\omega}_{O I}^{T} \hat{\boldsymbol{e}}}{r} \hat{\boldsymbol{r}}+\frac{g(r, \theta) \cos \theta \boldsymbol{\omega}_{O I}^{T} \hat{\boldsymbol{a}}}{r \sin \theta} \hat{\boldsymbol{e}}+\frac{\boldsymbol{\omega}_{O I}^{T}(\hat{\boldsymbol{r}} \sin \theta-\hat{\boldsymbol{a}} \cos \theta)}{\sin \theta} \hat{\boldsymbol{e}}
$$

Therefore, the product $\left.s_{a}(r, \theta) \dot{\hat{\boldsymbol{a}}}\right|_{\dot{\boldsymbol{r}}=\boldsymbol{h}}$ is bounded only if $\frac{s_{a}(r, \theta)}{\sin \theta}$ is bounded (which also implies that $s_{a}(r, \theta)$ is bounded). Therefore, if $\theta \rightarrow 0$ or $\theta \rightarrow \pi$, then it is required that $s_{a}(r, \theta) \rightarrow 0$ at least as quickly as $\sin \theta \rightarrow 0$. Note that this requirement is why the constraint C6) exists. If the speed can be arbitrarily fast near $\theta=\pi$, then that does not affect the stability, but does mean that the vector $\hat{\boldsymbol{a}}$ can change directions arbitrarily fast. The entire motivation for developing CLVF is to have a low-computation approach that limits the required acceleration, and so C6) had to be included.

Lastly, let us solve for constraints which ensure that $\boldsymbol{g}\left(\boldsymbol{r}, \hat{\boldsymbol{o}}, \boldsymbol{\omega}_{O I}\right)$ is Lipschitz time continuous. Again, assuming a bounded piecewise continuous derivative

$$
\Delta \boldsymbol{g}=\Delta\left(g(r, \theta) \boldsymbol{\omega}_{O I}^{\times} \hat{\boldsymbol{r}}\right)=\sum \int\left(\dot{g}(r, \theta) \boldsymbol{\omega}_{O I}^{\times} \hat{\boldsymbol{r}}+g(r, \theta) \dot{\boldsymbol{\omega}}_{O I}^{\times} \hat{\boldsymbol{r}}+g(r, \theta) \boldsymbol{\omega}_{O I}^{\times} \dot{\hat{\boldsymbol{r}}}\right) d t
$$

where $\left.\dot{g}(r, \theta)\right|_{\dot{\boldsymbol{r}}=\boldsymbol{h}}$ is already bounded, $\dot{\boldsymbol{\omega}}_{O I}$ must be bounded to be trackable, and 
$\left.\dot{\hat{\boldsymbol{r}}}\right|_{\dot{\boldsymbol{r}}=\boldsymbol{h}}$ is already bounded. Therefore, $\boldsymbol{g}$ is Lipschitz continuous if $g(r, \theta)$ is bounded. Because $\dot{\boldsymbol{d}}$ is the motion of a real object, it is also Lipschitz continuous. Furthermore, it is assumed for the use of CLVFs that $\ddot{\boldsymbol{d}}$ is relatively small compared to the acceleration capabilities of the controlled vehicle. Therefore, to guarantee an upper acceleration bound $u_{\max }$ on perfect tracking of the the simplified CLVF given by Eq. (3.72), it is sufficient that the following conditions are met:

E1) $v_{c}(r, \theta)$ is bounded and is Lipschitz continuous in $r$ and $\theta$;

E2) $g(r, \theta)$ is bounded and is Lipschitz continuous in $r$ and $\theta$. Furthermore, $\frac{g(r, \theta)}{r}$ is bounded.

E3) $s_{a}(r, \theta)$ is bounded and is Lipschitz continuous in $r$ and $\theta$. Furthermore, $\frac{s_{a}(r, \theta)}{r}$ and $\frac{s_{a}(r, \theta)}{\sin \theta}$ are bounded;

\subsubsection{Feed-Forward Acceleration Equations and Interpretation}

Utilizing the chain-rule on Eq. (3.72), we arrive at the following general feed-forward acceleration expression on the intervals where $\boldsymbol{h}$ is differentiable

$$
\dot{\boldsymbol{h}}\left(\boldsymbol{r}, \hat{\boldsymbol{o}}, \boldsymbol{\omega}_{O I}, \dot{\boldsymbol{d}}\right)=\dot{v}_{c} \hat{\boldsymbol{r}}+v_{c} \dot{\hat{\boldsymbol{r}}}+\dot{s}_{a} \hat{\boldsymbol{a}}+s_{a} \dot{\hat{\boldsymbol{a}}}+\dot{g} \boldsymbol{\omega}_{O I}^{\times} \hat{\boldsymbol{r}}+g \dot{\boldsymbol{\omega}}_{O I}^{\times} \hat{\boldsymbol{r}}+g \boldsymbol{\omega}_{O I}^{\times} \dot{\hat{\boldsymbol{r}}}+\ddot{\boldsymbol{d}}
$$

where arguments have been dropped for notational simplicity. In this work, it is considered necessary that perfect tracking of the desired guidance $\boldsymbol{h}$ respects the acceleration constraint of the controlled vehicle $u_{\max }$. Therefore, it is desirable to find a bound on feed-forward acceleration assuming perfect tracking, given by

$$
\begin{array}{r}
\left.\dot{\boldsymbol{h}}\left(\boldsymbol{r}, \hat{\boldsymbol{o}}, \boldsymbol{\omega}_{O I}, \dot{\boldsymbol{d}}\right)\right|_{\dot{\boldsymbol{r}}=\boldsymbol{h}}=\left(\left.\dot{v}_{c}\right|_{\dot{\boldsymbol{r}}=\boldsymbol{h}}\right) \hat{\boldsymbol{r}}+v_{c}\left(\left.\dot{\hat{\boldsymbol{r}}}\right|_{\dot{\boldsymbol{r}}=\boldsymbol{h}}\right)+\left(\left.\dot{s}_{a}\right|_{\dot{\boldsymbol{r}}=\boldsymbol{h}}\right) \hat{\boldsymbol{a}}+s_{a}\left(\left.\dot{\hat{\boldsymbol{a}}}\right|_{\dot{\boldsymbol{r}}=\boldsymbol{h}}\right)+ \\
\left(\left.\dot{g}\right|_{\dot{\boldsymbol{r}}=\boldsymbol{h}}\right) \boldsymbol{\omega}_{O I}^{\times} \hat{\boldsymbol{r}}+g \dot{\boldsymbol{\omega}}_{O I}^{\times} \hat{\boldsymbol{r}}+g \boldsymbol{\omega}_{O I}^{\times}\left(\left.\dot{\hat{\boldsymbol{r}}}\right|_{\dot{\boldsymbol{r}}=\boldsymbol{h}}\right)+\ddot{\boldsymbol{d}}
\end{array}
$$

As a consequence of the previous developments the derivatives under perfect track$\left.\operatorname{ing} \dot{\hat{\boldsymbol{r}}}\right|_{\dot{\boldsymbol{r}}=\boldsymbol{h}}$ and $\left.\dot{\hat{\boldsymbol{a}}}\right|_{\dot{\boldsymbol{r}}=\boldsymbol{h}}$ have already been solved, and are given by Eqs. (3.89) and (3.94) respectively. Further, the derivatives $\left.\dot{v}_{c}\right|_{\dot{\boldsymbol{r}}=\boldsymbol{h}},\left.\dot{s}_{a}\right|_{\dot{\boldsymbol{r}}=\boldsymbol{h}}$ and $\left.\dot{g}\right|_{\dot{\boldsymbol{r}}=\boldsymbol{h}}$ are attainable on their respective intervals of continuous differentiability (after they have been selected, of 
course) because equations for $\left.\dot{r}\right|_{\dot{\boldsymbol{r}}=\boldsymbol{h}}$ and $\left.\dot{\theta}\right|_{\dot{\boldsymbol{r}}=\boldsymbol{h}}$ have already been derived, and are given by Eqs. (3.83) and (3.86) respectively.

However, it may be easier to solve for a bound if Eq. (3.96) is given some familiar structure. Furthermore, some additional insight is available when the equation is rearranged. After substituting Eq. (3.89) and Eq. (3.94) into Eq. (3.97) and (tediously) simplifying, Eq. (3.97) can be put into the following form

$$
\begin{array}{r}
\left.\dot{\boldsymbol{h}}\left(\boldsymbol{r}, \hat{\boldsymbol{o}}, \boldsymbol{\omega}_{O I}, \dot{\boldsymbol{d}}\right)\right|_{\dot{\boldsymbol{r}}=\boldsymbol{h}}=\phi_{1}(r, \theta) \hat{\boldsymbol{r}}+\phi_{2}(r, \theta) \hat{\boldsymbol{a}}+\boldsymbol{\omega}_{O I}^{\times} \boldsymbol{\Omega}_{1}(\boldsymbol{r}, \hat{\boldsymbol{o}})+\boldsymbol{\Omega}_{2}\left(\boldsymbol{r}, \hat{\boldsymbol{o}}, \boldsymbol{\omega}_{O I}\right) \\
+\frac{g^{2}(r, \theta)}{r} \boldsymbol{\omega}_{O I}^{\times} \boldsymbol{\omega}_{O I}^{\times} \hat{\boldsymbol{r}}+g(r, \theta) \dot{\boldsymbol{\omega}}_{O I}^{\times} \hat{\boldsymbol{r}}+\ddot{\boldsymbol{d}}
\end{array}
$$

where

$$
\begin{gathered}
\phi_{1}(r, \theta)=\left.\dot{v}_{c}(r, \theta)\right|_{\dot{\boldsymbol{r}}=\boldsymbol{h}}-\frac{s_{a}^{2}(r, \theta)}{r} \\
\phi_{2}(r, \theta)=\left.\dot{s}_{a}(r, \theta)\right|_{\dot{\boldsymbol{r}}=\boldsymbol{h}}+\frac{s_{a}(r, \theta) v_{c}(r, \theta)}{r} \\
\boldsymbol{\Omega}_{1}(\boldsymbol{r}, \hat{\boldsymbol{o}})=\left(\left.\dot{g}(r, \theta)\right|_{\dot{\boldsymbol{r}}=\boldsymbol{h}}+\frac{g(r, \theta)}{r} v_{c}(r, \theta)\right) \hat{\boldsymbol{r}}+\left(s_{a}(r, \theta)+\frac{g(r, \theta)}{r} s_{a}(r, \theta)\right) \hat{\boldsymbol{a}} \\
\boldsymbol{\Omega}_{2}\left(\boldsymbol{r}, \hat{\boldsymbol{o}}, \boldsymbol{\omega}_{O I}\right)=s_{a}(r, \theta)\left(1-\frac{g(r, \theta)}{r}\right)\left(\hat{\boldsymbol{r}} \boldsymbol{\omega}_{O I}^{T} \hat{\boldsymbol{e}}-\frac{\cos \theta}{\sin \theta} \hat{\boldsymbol{e}} \boldsymbol{\omega}_{O I}^{T} \hat{\boldsymbol{a}}\right)
\end{gathered}
$$

Note that if $g(r, \theta)=r$ (which of course does not meet the acceleration constraint E2)), then Eqs. (3.101) and (3.102) reduce to

$$
\begin{gathered}
\boldsymbol{\Omega}_{1}(\boldsymbol{r}, \hat{\boldsymbol{o}})=2 v_{c}(r, \theta) \hat{\boldsymbol{r}}+2 s_{a}(r, \theta) \hat{\boldsymbol{a}} \\
\boldsymbol{\Omega}_{2}\left(\boldsymbol{r}, \hat{\boldsymbol{o}}, \boldsymbol{\omega}_{O I}\right)=\mathbf{0}
\end{gathered}
$$

By observation of the other terms, it is clear that a selection of $g(r, \theta)=r$ reduces the equations to the familiar form of feed-forward accelerations used for tracking within a rotating reference frame. Indeed, tracking the rotating reference frame upon arrival to $\mathbb{A}_{c}$ is the purpose of D2) (or equivalently C5)). Likewise, although not mandated, a well-designed set of functions $g(r, \theta)$ and $s_{a}(r, \theta)$ will approach 0 as $r \rightarrow \infty$. Therefore, 
very far from the target Eq. (3.98) simply becomes

$$
\left.\dot{\boldsymbol{h}}\left(\boldsymbol{r}, \hat{\boldsymbol{o}}, \boldsymbol{\omega}_{O I}, \dot{\boldsymbol{d}}\right)\right|_{\dot{\boldsymbol{r}}=\boldsymbol{h}}=\left(\left.\dot{v}_{c}(r, \theta)\right|_{\dot{\boldsymbol{r}}=\boldsymbol{h}}\right) \hat{\boldsymbol{r}}+\ddot{\boldsymbol{d}}
$$

which is a contraction in the inertial frame. Therefore, for values of $r$ between $\infty$ and $\alpha$, Eq. (3.98) is an acceleration-bounded feed-forward equation designed to transition from an inertial contraction into a body-fixed trajectory.

\subsubsection{Tracking the CLVF with Saturation}

Provided that no saturation occurs, Eq. (2.7) proposed by [17] is still a globally asymptotically stable controller for tracking CLVFs by the same proof (Theorem 2 in this paper).

However, although the Eqs. (3.98)-(3.102) may be used to ensure that $\|\dot{\boldsymbol{h}}\|_{\dot{\boldsymbol{r}}=\boldsymbol{h}}$ is bounded, there is no guarantee that $\|\dot{\boldsymbol{h}}\|$ is bounded. Furthermore, because this controller uses a constant gain $\beta$, it is apparent that for sufficiently large tracking error the acceleration commands will saturate. Therefore, it is worthwhile to study the effects of saturation on Theorem 2. First, let us assume the actual acceleration controller is given by

$$
\ddot{\boldsymbol{r}}= \begin{cases}\boldsymbol{u}, & \|\boldsymbol{u}\| \leq u_{\max }, \\ \left(\frac{u_{\max }}{\|\boldsymbol{u}\|}\right) \boldsymbol{u}, & \text { otherwise }\end{cases}
$$

where $\boldsymbol{u}$ is still defined as

$$
\boldsymbol{u}=-\beta(\dot{\boldsymbol{r}}-\boldsymbol{h})+\dot{\boldsymbol{h}}
$$

Let us return to the control Lyapunov function $W$ given by Eq. (2.8) with the time derivative given by Eq. (2.9). Substituting Eq. (3.106) into Eq. (2.9) assuming saturation gives

$$
\dot{W}=-\beta\left(\frac{u_{\max }}{\|\boldsymbol{u}\|}\right)(\dot{\boldsymbol{r}}-\boldsymbol{h})^{T}(\dot{\boldsymbol{r}}-\boldsymbol{h})-(\dot{\boldsymbol{r}}-\boldsymbol{h})^{T}\left(1-\frac{u_{\max }}{\|u\|}\right) \dot{\boldsymbol{h}}
$$


which has the potential to become positive if

$$
\left(1-\frac{u_{\max }}{\|u\|}\right)\|\dot{\boldsymbol{h}}\|>\beta\left(\frac{u_{\max }}{\|\boldsymbol{u}\|}\right)\|\dot{\boldsymbol{r}}-\boldsymbol{h}\|
$$

At this point, recall that one of the assumptions motivating CLVFs is that the controlled vehicle is initialized reasonably far away from the target, and therefore tracking the target attitude is unreasonable. If $g(r, \theta)$ and $s_{a}(r, \theta)$ are well-designed, it can be assumed that $g(r, \theta) \rightarrow 0$ and $s_{a}(r, \theta) \rightarrow 0$ as $r \rightarrow \infty$. The CLVF at a sufficiently large distances can therefore be approximated as

$$
\boldsymbol{h}\left(\boldsymbol{r}, \boldsymbol{o}, \boldsymbol{\omega}_{O I}, \dot{\boldsymbol{d}}\right) \approx v_{c}(r, \theta) \hat{\boldsymbol{r}}+\dot{\boldsymbol{d}}
$$

In the spacecraft case, so long as the target and chaser begin with a separation distance which is far less than the orbital radius, a non-rotating reference frame fixed to the target spacecraft's center of mass can be approximated as inertial from the chasers perspective, and $\dot{\boldsymbol{d}}=\mathbf{0}$ in this reference frame. Furthermore, it is known from E1) that $v_{c}(r, \theta)$ is bounded. It is reasonable to assume that $\left\|v_{c}(r, \theta)\right\|$ is maximized for large distances, and therefore $\dot{v}_{c}(r, \theta) \approx 0$. Therefore, knowledge of CLVF applications leads to the reasonable assumption that when the controller is initialized, the controlled vehicle will be distant from the target, and

$$
\dot{h} \approx 0
$$

regardless of velocity errors which may be present at initialization. This fact is illustrated in Fig. 3.3 for one particular case (the target is the same as that presented in Sec. 3.4). Note that, even for modest distances from the target and an initial velocity nearly perpendicular to the field direction, the desired velocity (which is represented by the nearest "blue arrow" to the chaser at any given time) varies very little across the first 24 seconds. Therefore, under standard initialization conditions, saturated thrusters result in the following approximate time-derivative of the control Lyapunov function

$$
\dot{W} \approx-u_{\max }(\dot{\boldsymbol{r}}-\boldsymbol{h})^{T}(\dot{\boldsymbol{r}}-\boldsymbol{h})
$$



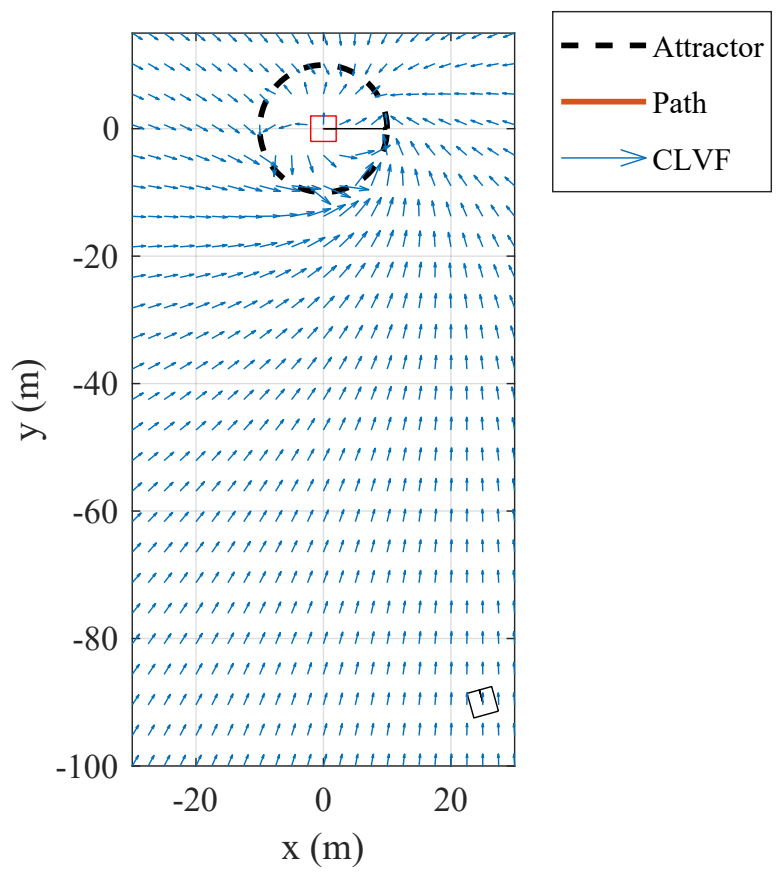

(a) $\mathrm{t}=0.00 \mathrm{~s}$.

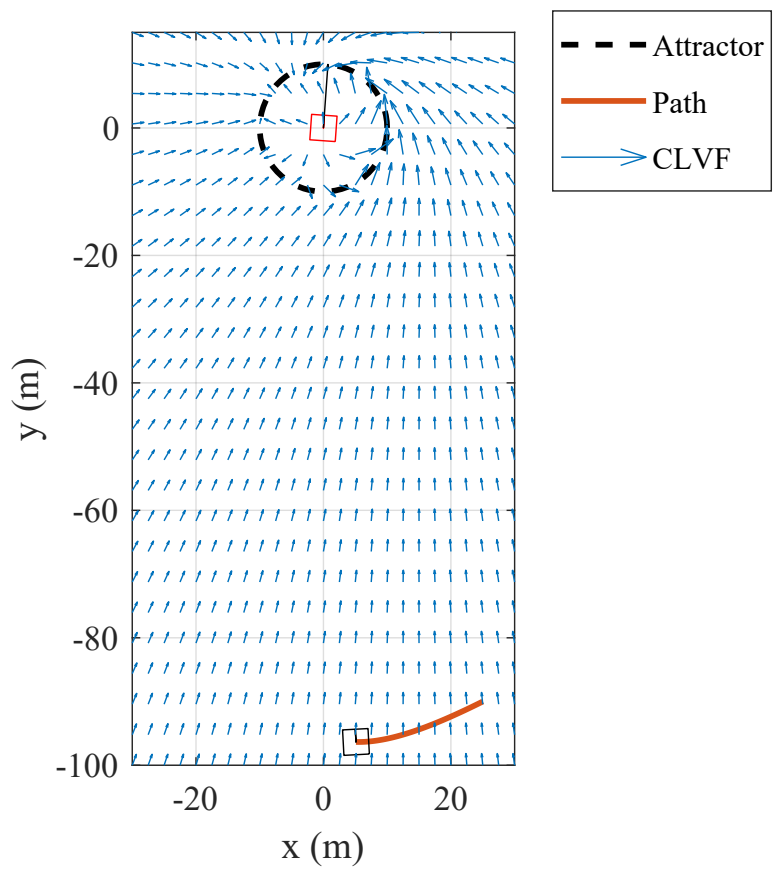

(b) $\mathrm{t}=24.00 \mathrm{~s}$.

Figure 3.3: Desired velocity remaining approximately constant from modest initial position separations. 
For reasonable values of $u_{\max }$, it may be assumed that the error dynamics decay below saturation levels well before the chaser and target are close enough together that Eq. (3.111) becomes a poor assumption.

\subsection{Design Procedure and Simulations}

In this Section, a full CLVF design is performed for a spacecraft performing a closeproximity inspection of a spinning target. The design is repeated for various values of $u_{\max }$, and results are presented for three different sets of initial conditions. The design procedure employed is as follows:

1. Design the functions $v_{c}(r, \theta), s_{a}(r, \theta)$ and $g(r, \theta)$ according to the desired behaviour while meeting stability constraints D1)-D3) and acceleration constraints E1)-E3). Leave some parameters in these functions unknown.

2. Using Eq.'s (3.98)-(3.102), solve for an acceleration bound as a function of the unknown parameters from step 1 ;

3. Using the acceleration bound function from step 2, perform a parameter search such that the bounded acceleration is less than or equal to $u_{\max }$.

4. Using the selected functions from step 1 with parameters from step 3, evaluate Eq. (3.55) to ensure that there is reasonable robustness to convergence errors. If not, return to step 3 and select more suitable parameters.

\subsubsection{Scenario Description}

For simplicity of presentation, let us assume in the following that the chaser spacecraft approaches the target spacecraft in the plane defined by the target spin vector (note that later Chapters will perform design examples for full three dimensional motion). The goal of the chaser is to contract to a radius of $\alpha=10 \mathrm{~m}$, and repetitively inspect each of the four sides of the target while it spins. An example time-lapse of this trajectory is presented in the target body-fixed frame in Fig. 3.4. The chaser spacecraft is set to observe each side of the target for 30 seconds. Between these 30 second pauses, the desired angle between the chaser and the target increases by $\pi / 2$ 


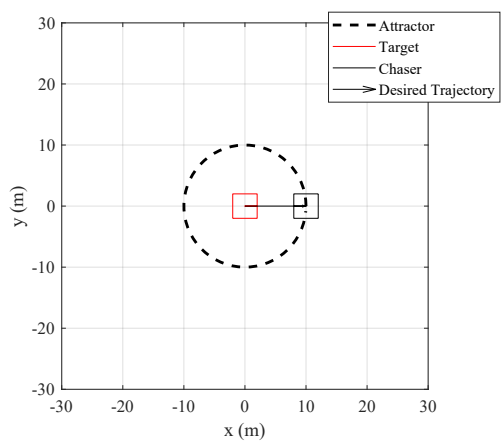

(a) Inspection of side 1 .

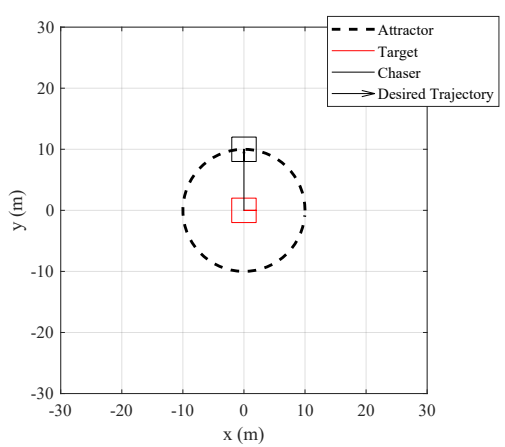

(c) Inspection of side 2 .

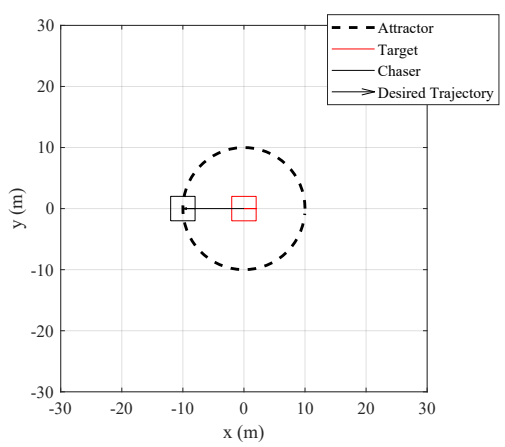

(e) Inspection of side 3 .

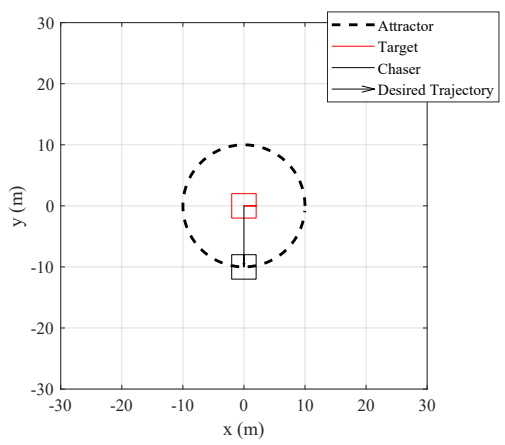

(g) Inspection of side 4 .

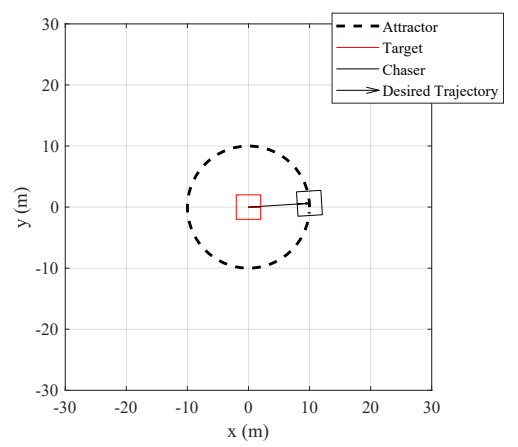

(b) Switching to side 2 .

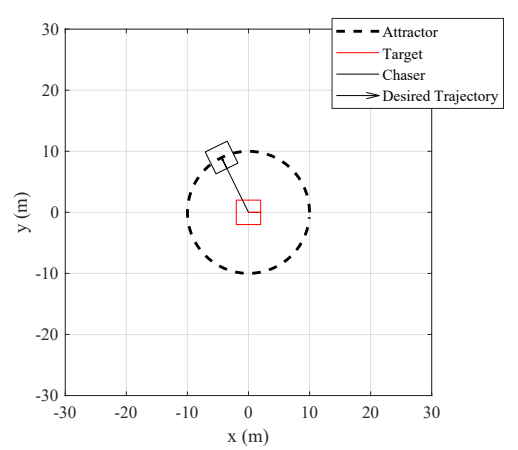

(d) Switching to side 3 .

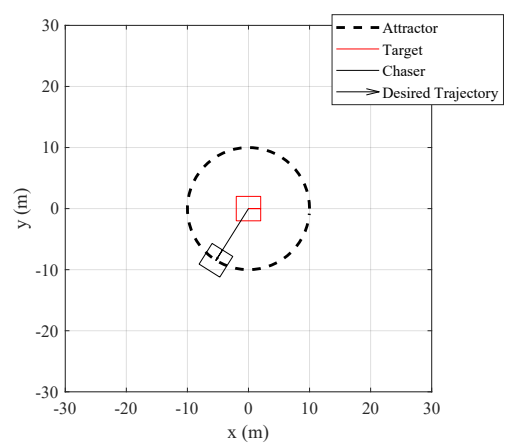

(f) Switching to side 4 .

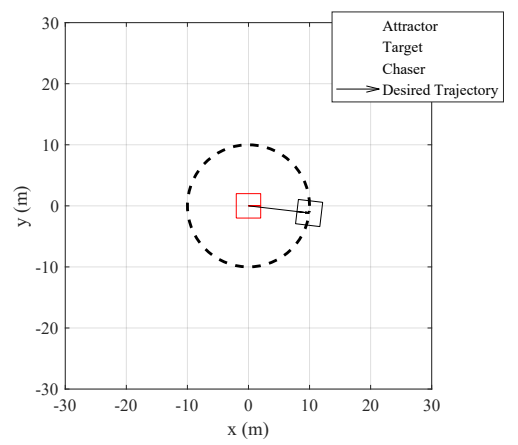

(h) Switching to side 1 .

Figure 3.4: Desired inspection trajectory. 
radians over a 30 second period. That is, the rotation of the desired trajectory in the body-fixed frame is

$$
\omega_{O B}(t)= \begin{cases}0.0 \mathrm{rads} / \mathrm{s}, & \text { during inspections } \\ \left(0.01047 t_{d}-3.4907(e-04) t_{d}^{2}\right) \mathrm{rads} / \mathrm{s}, & \text { when changing inspection angle }\end{cases}
$$

where $t_{d}$ is the time that has elapsed since departing the previous inspection angle. The spin rate of the target spacecraft is constant, defined as

$$
\omega_{B I}=0.1 \mathrm{rads} / \mathrm{s}
$$

\subsubsection{Design of the CLVF}

Let us now perform the design procedure according to the steps outlined at the start of this section.

\section{Design of speed and distance functions}

The following function is selected for $g(r, \theta)$

$$
g(r)= \begin{cases}r, & r<\alpha \\ \frac{\alpha^{2}}{r}, & \text { otherwise }\end{cases}
$$

which clearly meets the requirements of D2) and E2). This function also has the property that $g(\infty)=0$, which is beneficial for fuel expenditure. Furthermore, this selection will simplify finding an acceleration bound, as when the chaser is tracking perfectly and approaching from outside of the attractor, it can be seen that

$$
\left.\dot{g}(r)\right|_{\dot{\boldsymbol{r}}=\boldsymbol{h}}=-\frac{g(r)}{r} v_{c}(r, \theta)
$$


which cancels a term in Eq. (3.101). Next, following the constraints of D3) and E3), let us select $s_{a}(r, \theta)$ as

$$
s_{a}(r, \theta)= \begin{cases}k_{a} \frac{r}{\alpha} \sin \theta, & r<\alpha \\ k_{a} \frac{\alpha}{r} \sin \theta, & \text { otherwise. }\end{cases}
$$

where, following the design procedure step $1, k_{a}$ is an unselected strictly positive parameter. Likewise, let us select the velocity function which meets D1) and E1) as

$$
v_{c}(r)= \begin{cases}k_{c} \frac{\alpha-r}{b}, & \|\alpha-r\|<b \\ k_{c} \operatorname{sgn}(\alpha-r), & \text { otherwise. }\end{cases}
$$

where $k_{c}$ and $b$ are unselected strictly positive parameters. Therefore, by the constraints D1)-D3) the CLVF will inherit asymptotic stability, and by E1)-E3) there will be an upper bound on the required acceleration for perfect tracking. By finding a suitable $k_{a}, k_{c}$ and $b$, the upper bound on tracking acceleration for this field can adjusted to meet $u_{\max }$.

\section{Finding a function for the upper bound in terms of $k_{a}, k_{c}$ and $b$}

In general, the acceleration bound when approaching from the inside of the attractor $\mathbb{A}$ will not equal to the acceleration bound when approaching from the outside. Because it is assumed that the chaser spacecraft approaches from the outside, this is the bound we will search for.

To begin, let us make some simplifications to Eq. (3.98). First, the selection of $g(r)$ will cancel a term in Eq. (3.101) as previously discussed. Secondly, because both spacecraft experience roughly equal acceleration due to gravity, the acceleration of the target is approximated by $\ddot{\boldsymbol{d}} \approx \mathbf{0}$. Lastly, because the scenario is planar, $\boldsymbol{\omega}_{O I}$ will be entirely along the $\hat{\boldsymbol{e}}$ direction. Making these simplifications and rearranging, Eq. 
(3.98) reduces to

$$
\left.\dot{\boldsymbol{h}}\left(\boldsymbol{r}, \hat{\boldsymbol{o}}, \boldsymbol{\omega}_{O I}, \dot{\boldsymbol{d}}\right)\right|_{\dot{\boldsymbol{r}}=\boldsymbol{h}}=\left(\left.\dot{v}_{c}\right|_{\dot{\boldsymbol{r}}=\boldsymbol{h}}-\frac{\left(s_{a}+g \omega_{O I}\right)^{2}}{r}\right) \hat{\boldsymbol{r}}+\left(\left.\dot{s}_{a}\right|_{\dot{\boldsymbol{r}}=\boldsymbol{h}}+\frac{s_{a} v_{c}}{r}+g \dot{\omega}_{O I}\right) \hat{\boldsymbol{a}}
$$

where arguments are dropped for simplicity. After some straight-forward time-derivatives of each function (assuming perfect tracking) and substitution into Eq. (3.119), the following somewhat conservative bound is attained

$$
\|\boldsymbol{h}\|_{\dot{\boldsymbol{r}}=\boldsymbol{h}}<\left(\left[\frac{k_{c}^{2}}{b}+\frac{\left(k_{a}+\alpha \omega_{\max }\right)^{2}}{\alpha}\right]^{2}+\left[\frac{k_{a}^{2}}{2 \alpha}+k_{a} \omega_{\max }+\alpha \dot{\omega}_{\max }\right]^{2}\right)^{1 / 2}
$$

where $\omega_{\max }$ and $\dot{\omega}_{\max }$ are the maximum norms of the vectors $\boldsymbol{\omega}_{O I}$ and $\dot{\boldsymbol{\omega}}_{O I}$ respectively. That is, for the general tumbling case

$$
\begin{aligned}
& \omega_{\max }=\left\|\boldsymbol{\omega}_{B I}^{B}+\boldsymbol{C}_{B O} \boldsymbol{\omega}_{O B}^{O}\right\|_{\text {max }} \\
& \dot{\omega}_{\max }=\left\|\dot{\boldsymbol{\omega}}_{B I}^{B}+\boldsymbol{C}_{B O} \dot{\boldsymbol{\omega}}_{O B}^{O}\right\|_{\text {max }}
\end{aligned}
$$

Alternatively, given that the chaser spacecraft has an acceleration limit $u_{\max }$, we want to find a set of parameters $\left(k_{a}, k_{c}, b\right)$ such that the following inequality is satisfied

$$
u_{\max } \geq\left(\left[\frac{k_{c}^{2}}{b}+\frac{\left(k_{a}+\alpha \omega_{\max }\right)^{2}}{\alpha}\right]^{2}+\left[\frac{k_{a}^{2}}{2 \alpha}+k_{a} \omega_{\max }+\alpha \dot{\omega}_{\max }\right]^{2}\right)^{1 / 2}
$$

It is useful to note that over the domain where $k_{a}, k_{c}$ and $b$ are all strictly greater than 0, Eq. (3.123) forms a convex set, and therefore any of the widely known convex search algorithms can be used in the selection of parameters.

\section{Selection of parameters according to the acceleration limit $u_{\max }$}

For simplicity, a line-search is used as the parameter selection algorithm; details beyond what is presented here for this algorithm, including alternative versions of the line-search, can be found in [28]. Note that a more advanced convex optimization method would likely result in better parameter selections, but that is beyond of scope 
of this Chapter. Initial search values for the parameters are taken as $k_{a}=0.5 \mathrm{~m} / \mathrm{s}$, $k_{c}=0.0 \mathrm{~m} / \mathrm{s}$ and $b=5 \mathrm{~m}$. The line-search direction was arbitrarily selected as

$$
\left[\begin{array}{lll}
\Delta k_{a} & \Delta k_{c} & \Delta b
\end{array}\right]=\left[\begin{array}{lll}
0.1 & 0.1 & -0.5
\end{array}\right] \gamma
$$

where $\gamma$ is some varying step size. This line is searched (following the algorithm outlined in [28]) until a combination $\left(k_{a}, k_{c}, b\right)$ is found such that Eq. (3.123) holds with equality. Of course, if the bound described by Eq. (3.123) is very loose, then the actual acceleration required to track the resulting CLVF may be far less than $u_{\max }$. Therefore, to take full advantage of the available thrust, the designer should use all available information to find a bound which is as tight as possible. Of course, some looseness in the bound does provide margin before the thrusters saturate (discussed in Sec. 3.3.4), which may be advantageous in many practical scenarios.

By considering multiple values for $u_{\max }$, Table 3.1 summarizes the resulting parameters from the line-search.

Table 3.1: Parameter selection for spacecraft with varying acceleration constraints.

\begin{tabular}{cccc}
\hline \hline$u_{\max }\left(\mathrm{m} / \mathrm{s}^{2}\right)$ & $k_{a}(\mathrm{~m} / \mathrm{s})$ & $k_{c}(\mathrm{~m} / \mathrm{s})$ & $b(\mathrm{~m})$ \\
\hline 5.0 & 1.454 & 0.9537 & 0.2315 \\
3.0 & 1.414 & 0.9139 & 0.4303 \\
1.0 & 1.0495 & 0.5495 & 2.253 \\
0.8 & 0.8634 & 0.3634 & 3.183 \\
0.7 & 0.7226 & 0.2336 & 3.832 \\
\hline \hline
\end{tabular}

\section{Evaluating robustness to convergence errors}

To evaluate the robustness of convergence errors, let us begin with Eq. (3.55)

$$
\Delta \theta \leq\left(\frac{\left\|\frac{\partial \psi}{\partial \boldsymbol{r}}\left(\boldsymbol{r}^{*}, \boldsymbol{o}, \cdot\right)\right\|}{\alpha\left\|\frac{\partial V_{a}}{\partial \boldsymbol{r}}\left(\boldsymbol{r}^{*}, \boldsymbol{o}, \cdot\right) \frac{\partial \boldsymbol{a}}{\partial \boldsymbol{r}}\left(\boldsymbol{r}^{*}, \boldsymbol{o}, \cdot\right)\right\|}\right)\left\|\Delta \boldsymbol{r}_{n}\right\|
$$

The specific steps for evaluating these functions are not necessary for the understanding of what follows, and so the details are left to Appendix A. The resulting robustness 
bound is found as

$$
\frac{\Delta \theta}{\left\|\Delta \boldsymbol{r}_{n}\right\|} \leq \frac{2 \omega_{\max }}{k_{a}}
$$

Given that $\omega_{\max }=0.17854 \mathrm{rads} / \mathrm{s}$ in this scenario, Table 3.2 summarizes the alignment robustness for each $u_{\max }$ and corresponding $k_{a}$ from Table 3.1. For intuition, the third column gives the conservative thickness estimates (in degrees) of the regions surrounding the stable and unstable equilibrium point where instability becomes possible given a $10 \mathrm{~cm}$ contraction error. For the purpose of this work, each of these values are sufficiently robust, but could be lowered by solving for a higher $k_{a}$ in step 3.

Table 3.2: Alignment robustness to contraction errors for each $u_{\max }$.

\begin{tabular}{ccc}
\hline \hline$u_{\max }\left(\mathrm{m} / \mathrm{s}^{2}\right)$ & $\Delta \theta /\left\|\Delta \boldsymbol{r}_{n}\right\|(\mathrm{rads} / \mathrm{m})$ & $\Delta \theta$ for $\|\Delta \boldsymbol{r}\|=0.1 \mathrm{~m}\left(^{\circ}\right)$ \\
\hline 5.0 & 0.2456 & 1.41 \\
3.0 & 0.2525 & 1.45 \\
1.0 & 0.3402 & 1.95 \\
0.8 & 0.4136 & 2.37 \\
0.7 & 0.4624 & 2.65 \\
\hline
\end{tabular}

\subsubsection{Results}

For each acceleration constraint and corresponding parameter selections from Table 3.1, three different initial conditions are tested, the details of which are listed in Table 3.3. These conditions vary the chaser vehicles initial position and velocity, and also vary the initial angle of the target in the world frame, and the initial angle of $\hat{\boldsymbol{o}}^{B}$ relative to the $x$ vector of the target body-fixed frame. In every case, the controller gain value was selected as $\beta=0.05$. This small value is selected because after the chaser is tracking the CLVF, most of the control input is handled by the feed-forward acceleration equations. In a more realistic scenario with perturbations and imperfect measurements, a larger value for $\beta$ may be required. 
Table 3.3: Initial conditions.

\begin{tabular}{ccccc}
\hline \hline Case & Init Pos $(\mathrm{m})$ & Init Vel $\left(\mathrm{m} / \mathrm{s}^{2}\right)$ & Init Targ Ang (rad) & Init Traj Ang (rad) \\
\hline 1 & {$\left[\begin{array}{ll}-20 & 30\end{array}\right]^{T}$} & {$\left[\begin{array}{ll}0 & 0\end{array}\right]^{T}$} & 0 & $\pi$ \\
2 & {$\left[\begin{array}{ll}20 & -15\end{array}\right]^{T}$} & {$\left[\begin{array}{cc}-0.8 & -0.5\end{array}\right]^{T}$} & $-\pi / 4$ & 0 \\
3 & {$\left[\begin{array}{ll}30 & 15\end{array}\right]^{T}$} & {$\left[\begin{array}{ll}0 & 0\end{array}\right]^{T}$} & 0 & 0 \\
\hline \hline
\end{tabular}

Figures 3.5-3.9 show the resulting acceleration, convergence in $r$, and convergence in $\theta$ for each acceleration constraint and set of initial conditions. For Case 1, timelapses of the chaser spacecraft with an acceleration constraint of $u_{\max }=5 \mathrm{~m} / \mathrm{s}^{2}$ and $u_{\max }=0.7 \mathrm{~m} / \mathrm{s}^{2}$ are provided respectively in Fig. 3.10 and 3.11 .

As intended, for each case the actual acceleration stays within the vehicle acceleration constraint $u_{\max }$. Furthermore, the expected behaviour of convergence in $r$ followed by convergence in $\theta$ occurred for every value of $u_{\max }$ and under every tested set of initial conditions. However, for higher values of $u_{\max }$ the parameter search resulted in CLVFs where there was potential for more agile maneuvering, resulting in faster convergence. Intuitively, this means that vehicles with higher acceleration capabilities have a broader parameter selection to choose from, giving the opportunity for a design trade-off between maneuver duration and fuel expenditure. Indeed, the time-lapses in Fig. 3.10 and 3.11 show that the $u_{\max }=5 \mathrm{~m} / \mathrm{s}^{2}$ vehicle selects a path which converges much faster than the $u_{\max }=0.7 \mathrm{~m} / \mathrm{s}^{2}$ vehicle under identical initial conditions. Of course, finding a tighter bound than Eq. (3.123) would result in a larger set of permissible parameters for any given $u_{\max }$, resulting in more design options and potentially fuller use of the vehicles' acceleration capabilities.

By comparing the acceleration norm results under different initial conditions for the same set of CLVF parameters (for example, Figs. 3.5c and 3.5e), it is clear that the CLVF approach does not always use the full acceleration capabilities of the controlled vehicle. Rather, this approach designs the field such that under worst case conditions the total acceleration commands can never rise above the constraints set by the vehicle. Sometimes, particularly good conditions will occur, and the chaser will use very little of the acceleration capabilities, such as in Fig. 3.7a. Unfortunately, it is 


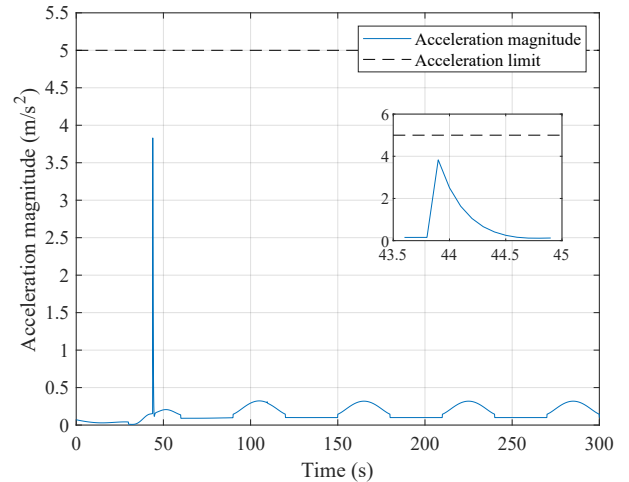

(a) Acc. norm over time - case 1 .

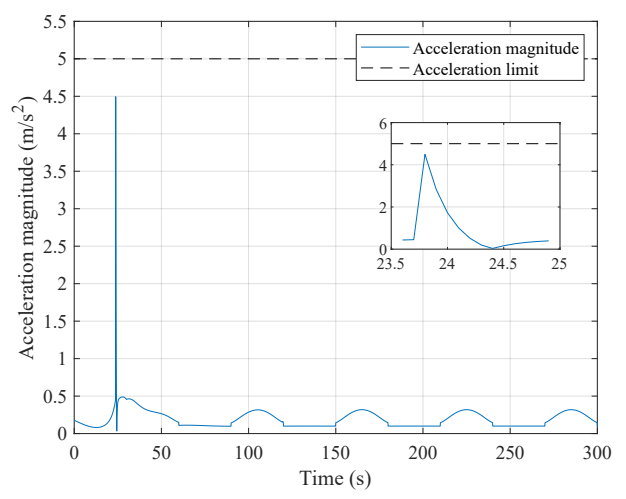

(c) Acc. norm over time - case 2 .

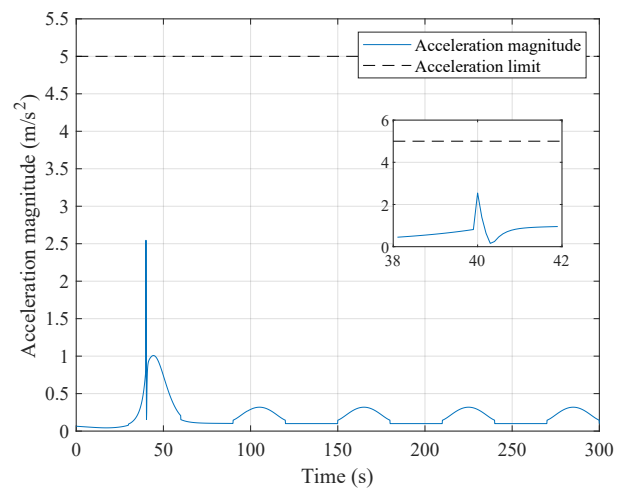

(e) Acc. norm over time - case 3.
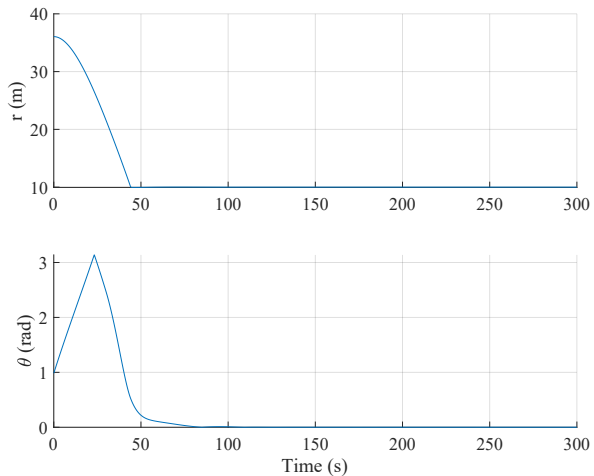

(b) $r$ and $\theta$ over time - case 1 .
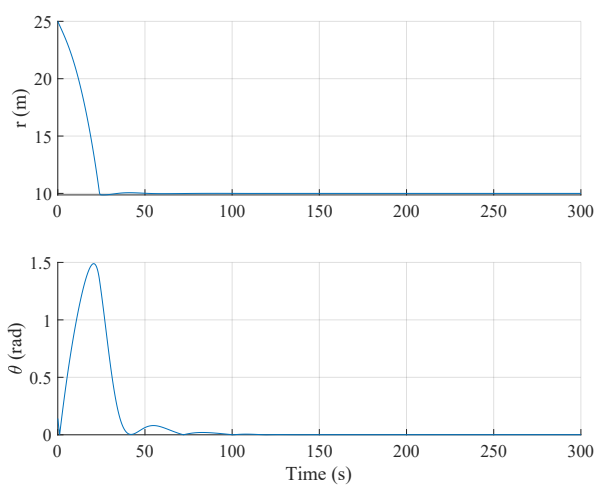

(d) $r$ and $\theta$ over time - case 2 .
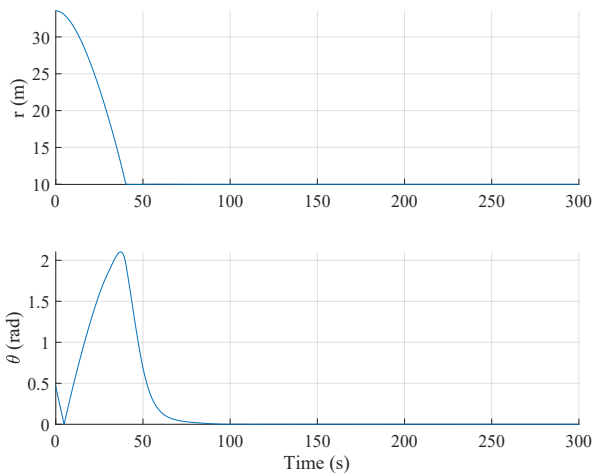

(f) $r$ and $\theta$ over time - case 3 .

Figure 3.5: Maximum acceleration constraint: $u_{\max }=5.0 \mathrm{~m} / \mathrm{s}^{2}$. 


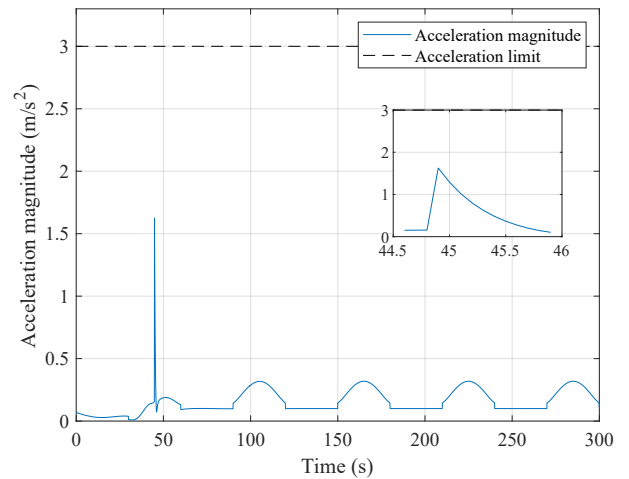

(a) Acc. norm over time - case 1.

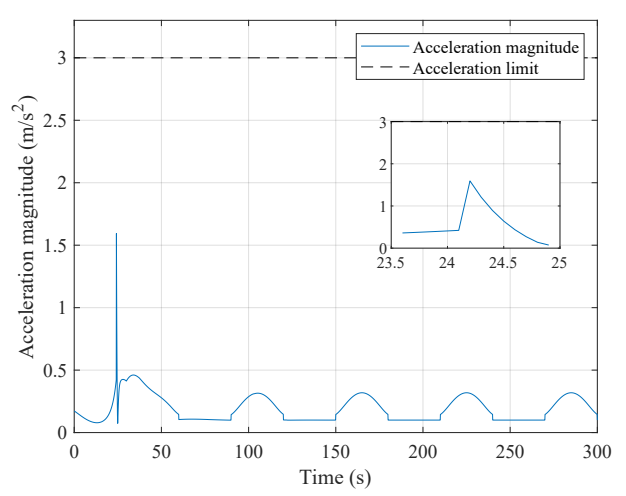

(c) Acc. norm over time - case 2 .

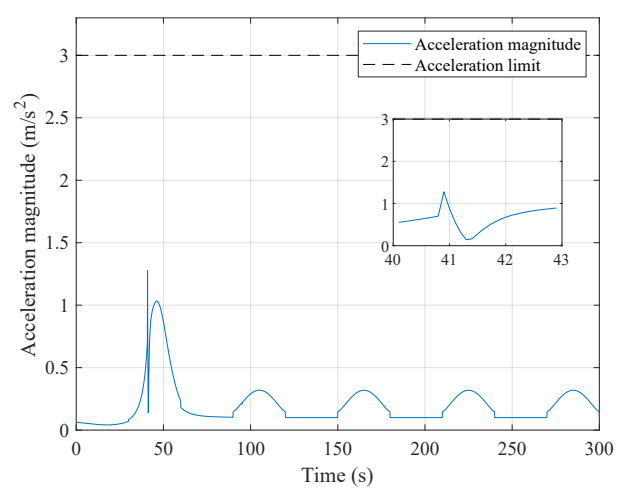

(e) Acc. norm over time - case 3.
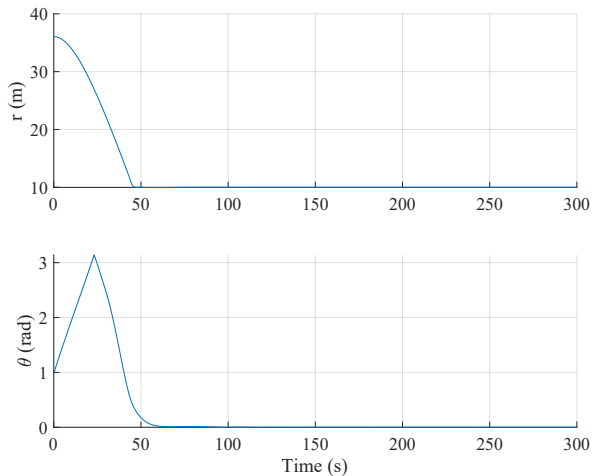

(b) $r$ and $\theta$ over time - case 1 .
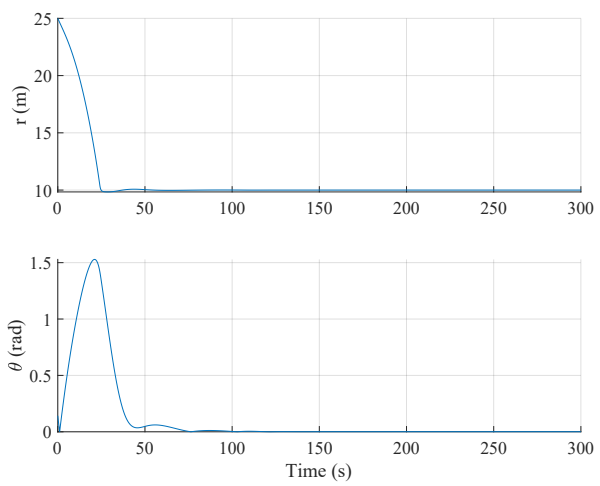

(d) $r$ and $\theta$ over time - case 2 .
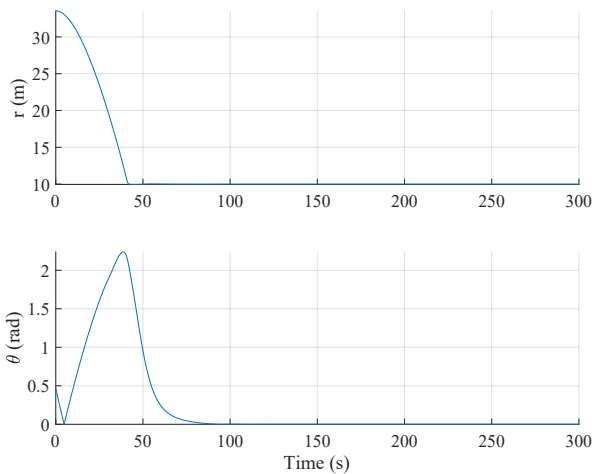

(f) $r$ and $\theta$ over time - case 3 .

Figure 3.6: Maximum acceleration constraint: $u_{\max }=3.0 \mathrm{~m} / \mathrm{s}^{2}$. 


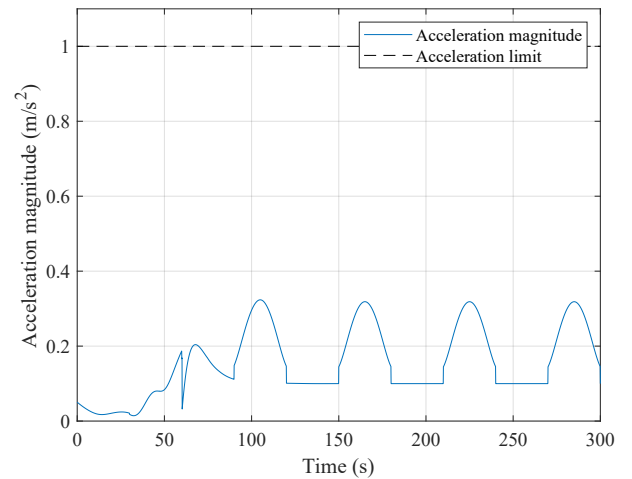

(a) Acc. norm over time - case 1.

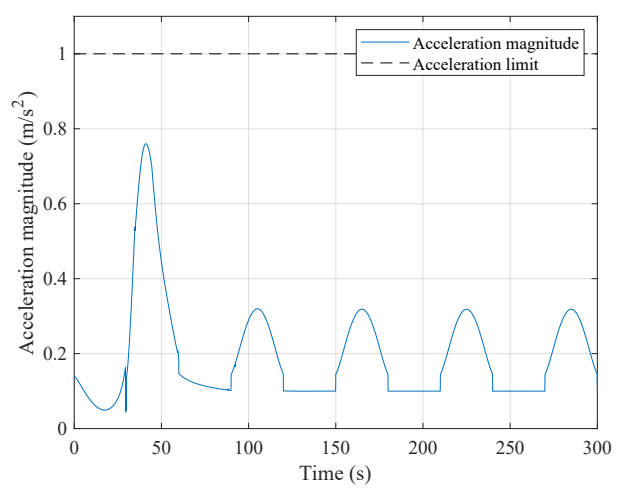

(c) Acc. norm over time - case 2 .

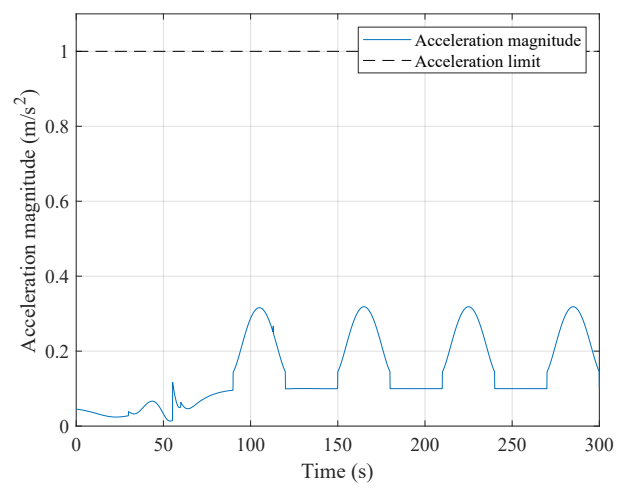

(e) Acc. norm over time - case 3.
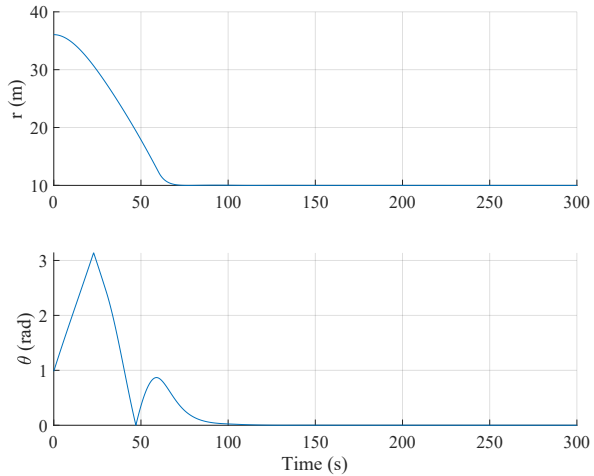

(b) $r$ and $\theta$ over time - case 1 .
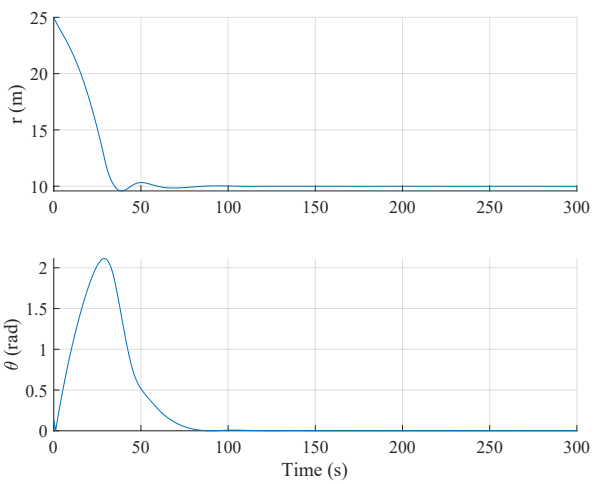

(d) $r$ and $\theta$ over time - case 2 .
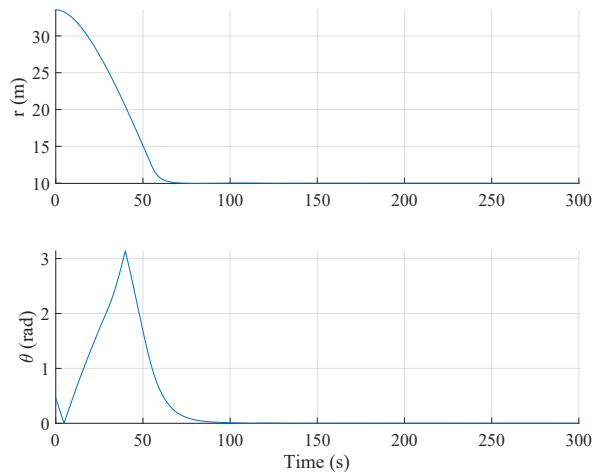

(f) $r$ and $\theta$ over time - case 3 .

Figure 3.7: Maximum acceleration constraint: $u_{\max }=1.0 \mathrm{~m} / \mathrm{s}^{2}$. 


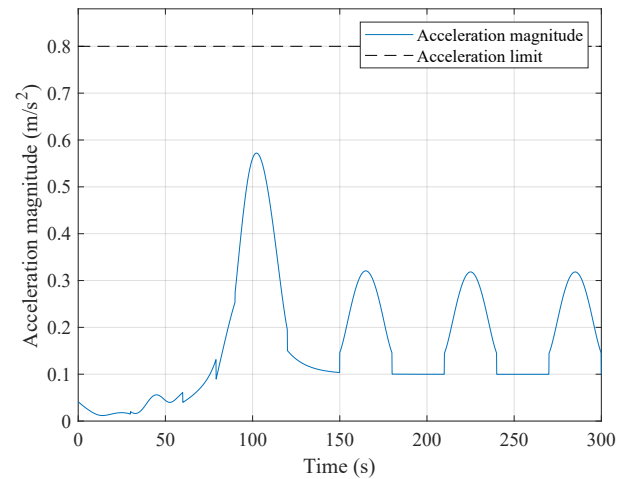

(a) Acc. norm over time - case 1 .
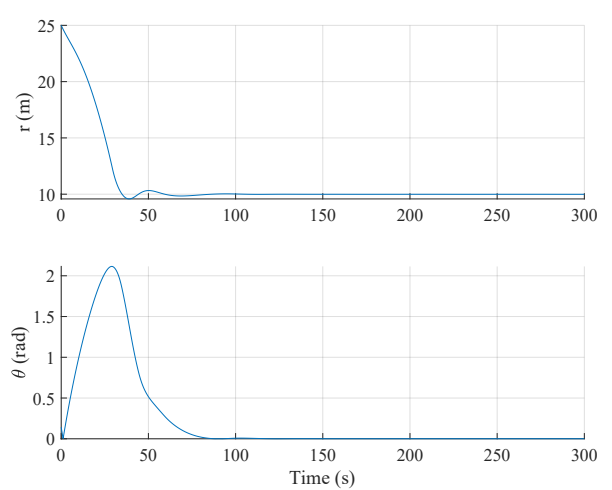

(c) Acc. norm over time - case 2 .
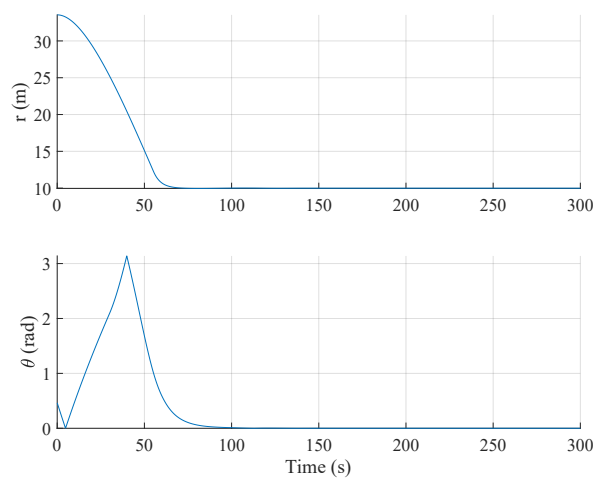

(e) Acc. norm over time - case 3 .
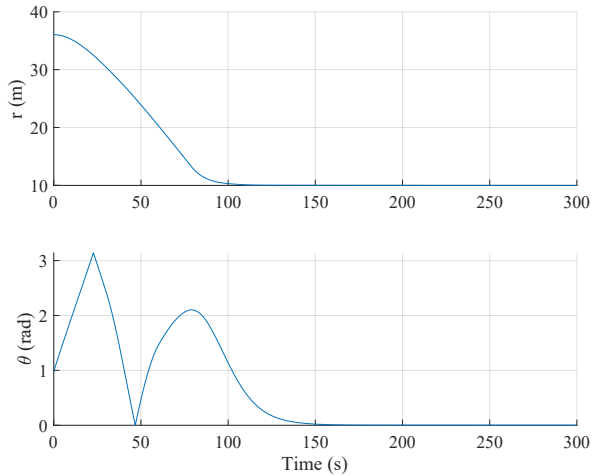

(b) $r$ and $\theta$ over time - case 1 .
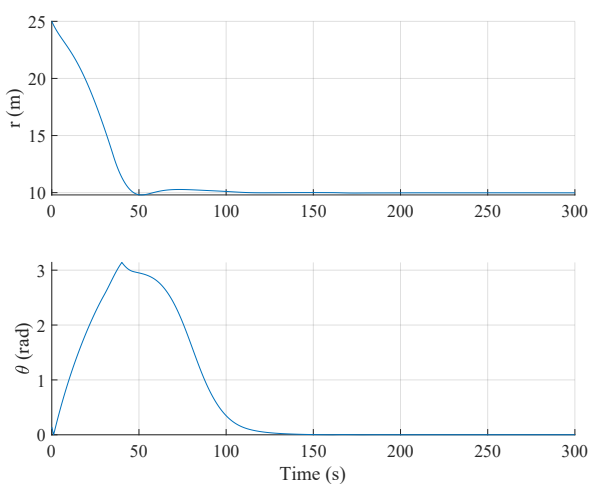

(d) $r$ and $\theta$ over time - case 2 .
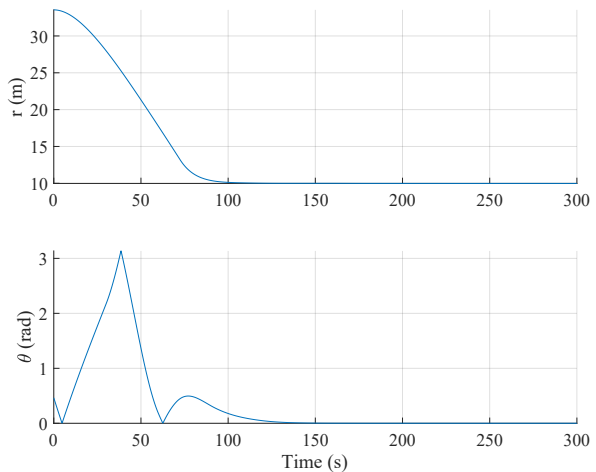

(f) $r$ and $\theta$ over time - case 3 .

Figure 3.8: Maximum acceleration constraint: $u_{\max }=0.80 \mathrm{~m} / \mathrm{s}^{2}$. 


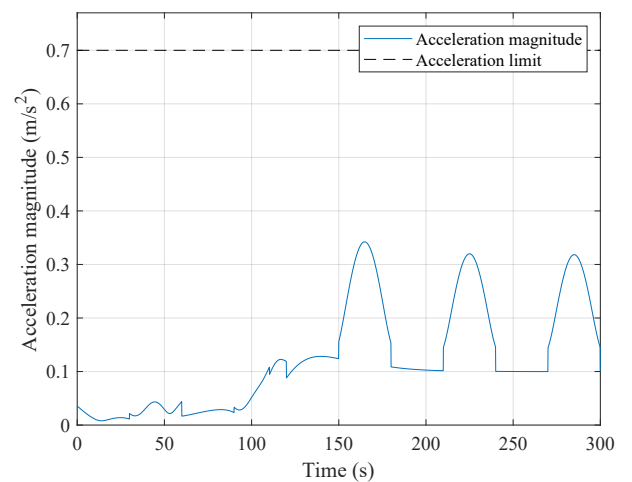

(a) Acc. norm over time - case 1 .

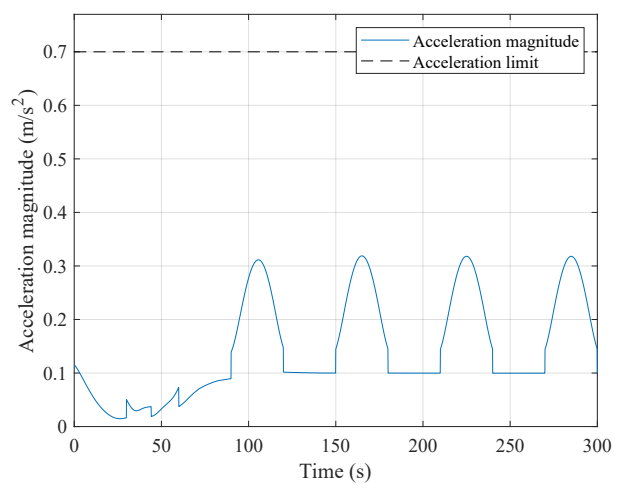

(c) Acc. norm over time - case 2 .

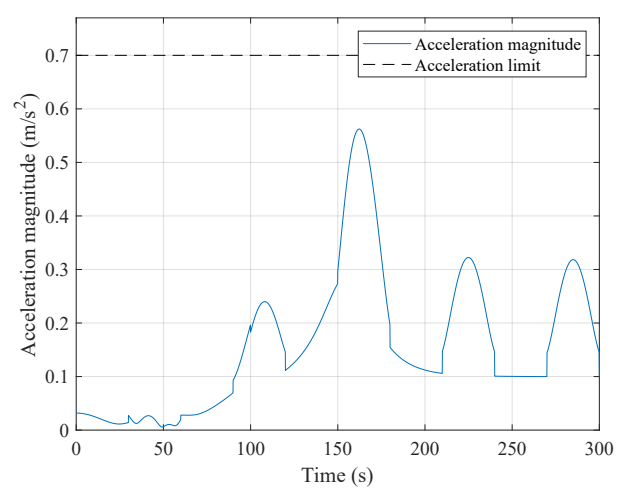

(e) Acc. norm over time - case 3.
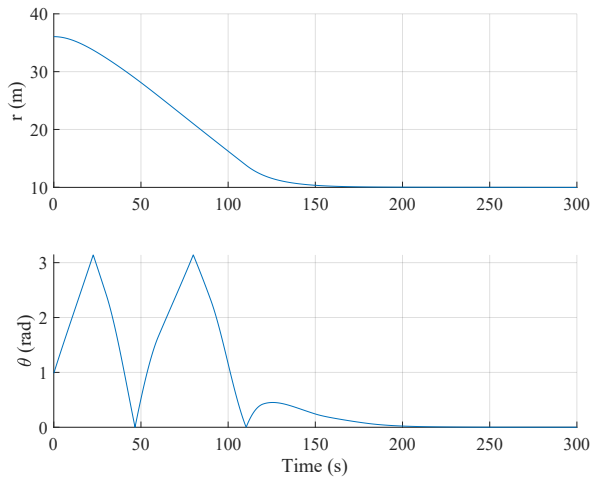

(b) $r$ and $\theta$ over time - case 1 .
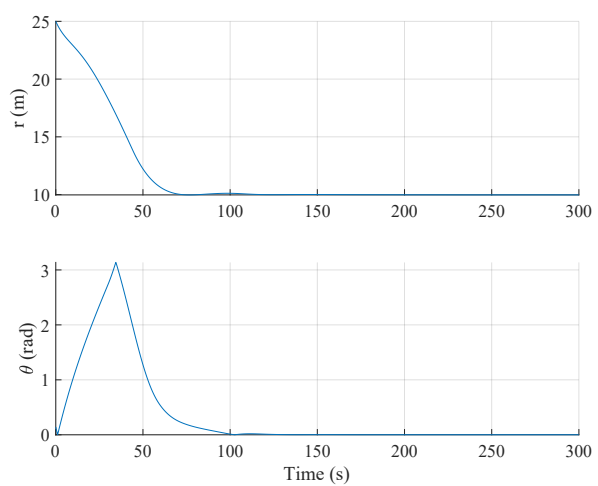

(d) $r$ and $\theta$ over time - case 2 .
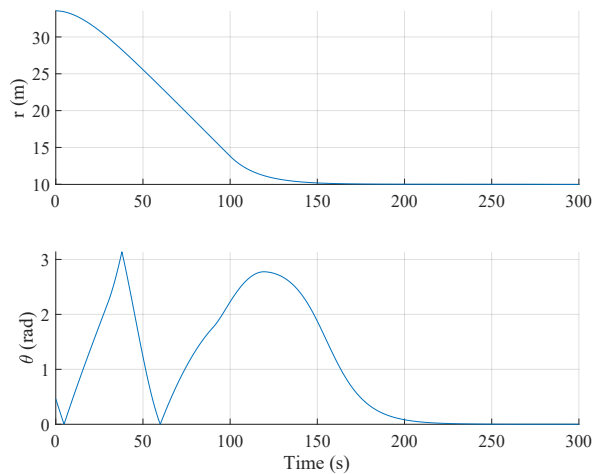

(f) $r$ and $\theta$ over time - case 3 .

Figure 3.9: Maximum acceleration constraint: $u_{\max }=0.70 \mathrm{~m} / \mathrm{s}^{2}$. 


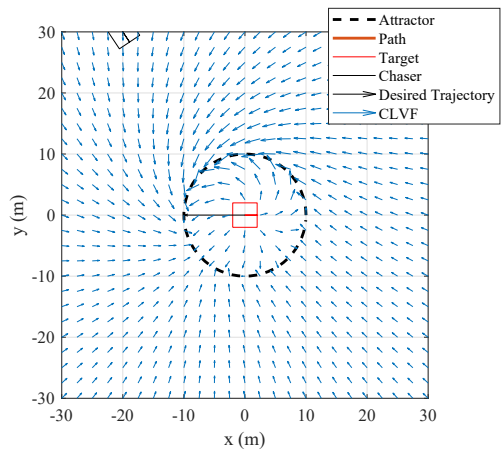

(a) $\mathrm{t}=0.00 \mathrm{~s}$.

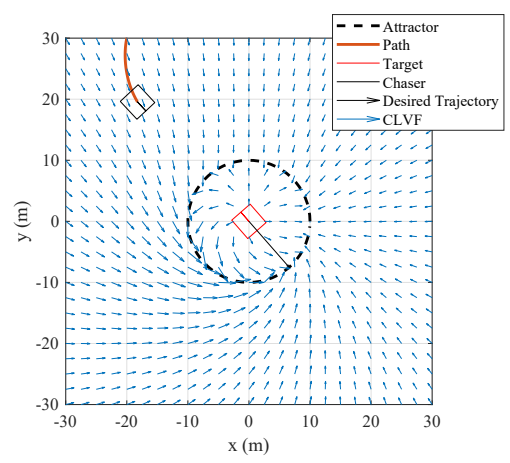

(c) $\mathrm{t}=22.86 \mathrm{~s}$.

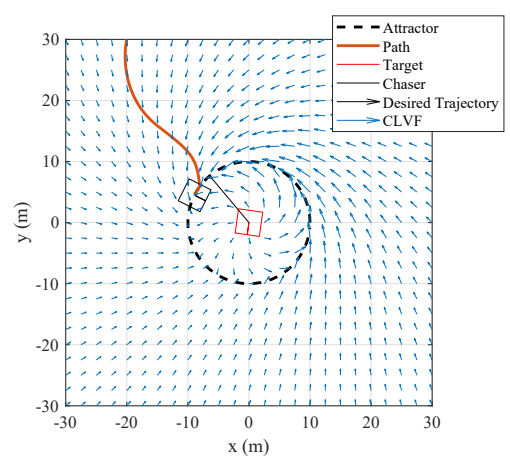

(e) $\mathrm{t}=45.71 \mathrm{~s}$.

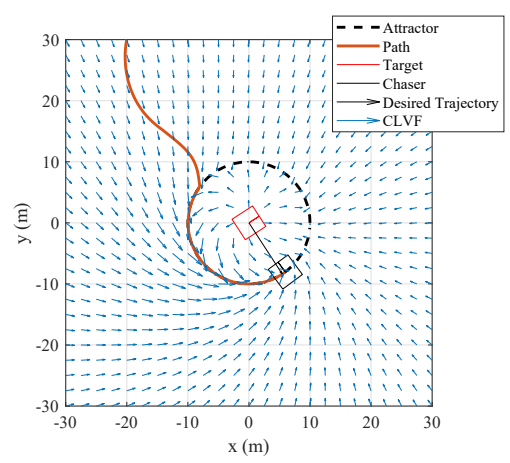

(g) $\mathrm{t}=68.57 \mathrm{~s}$.

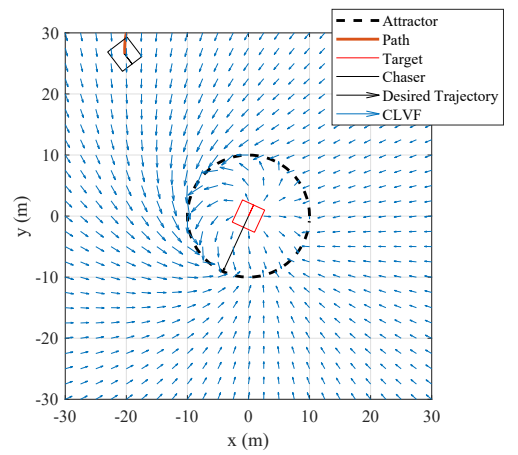

(b) $\mathrm{t}=11.43 \mathrm{~s}$.

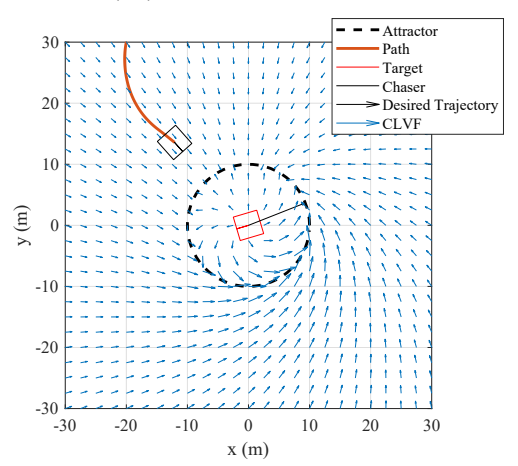

(d) $\mathrm{t}=34.29 \mathrm{~s}$.

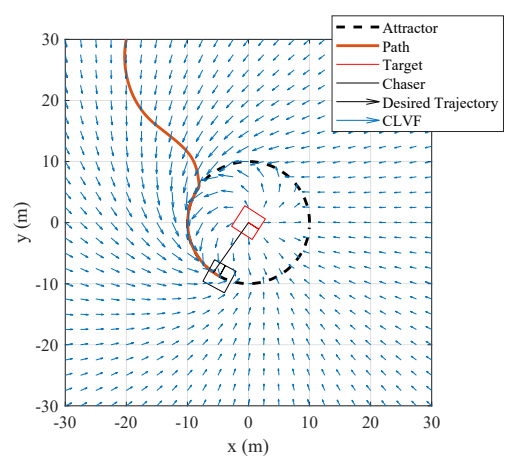

(f) $\mathrm{t}=57.14 \mathrm{~s}$.

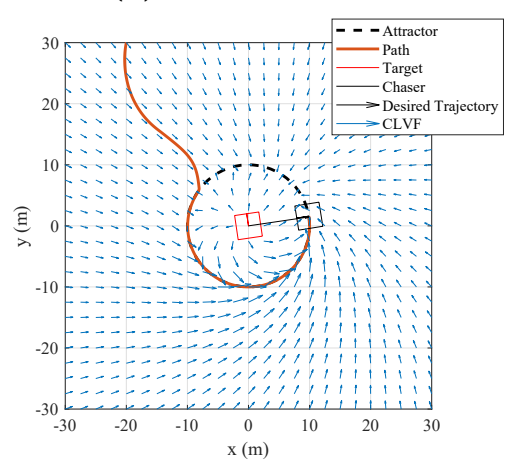

(h) $\mathrm{t}=80.00 \mathrm{~s}$.

Figure 3.10: Time-lapse of $u_{\max }=5.0 \mathrm{~m} / \mathrm{s}^{2}$ - case 1 . 


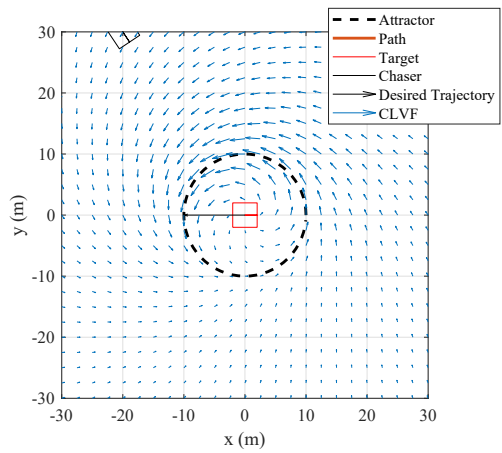

(a) $\mathrm{t}=0.00 \mathrm{~s}$.

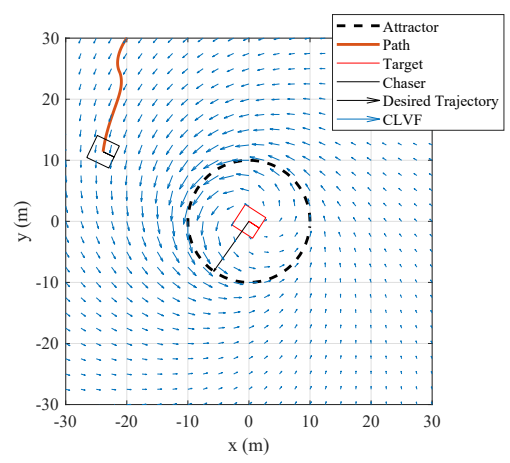

(c) $\mathrm{t}=57.14 \mathrm{~s}$.

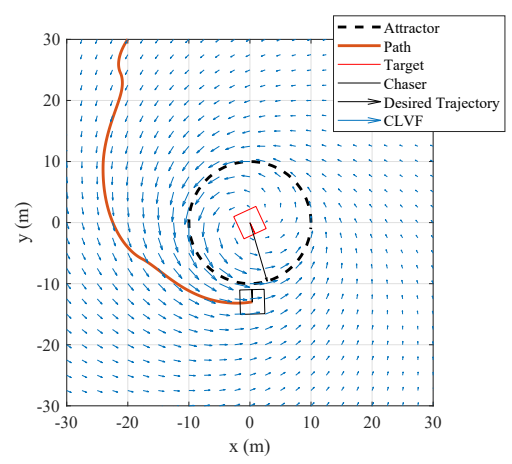

(e) $\mathrm{t}=114.28 \mathrm{~s}$.

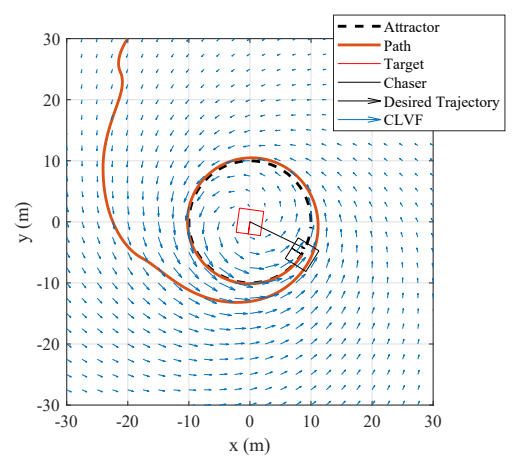

(g) $\mathrm{t}=171.43 \mathrm{~s}$.

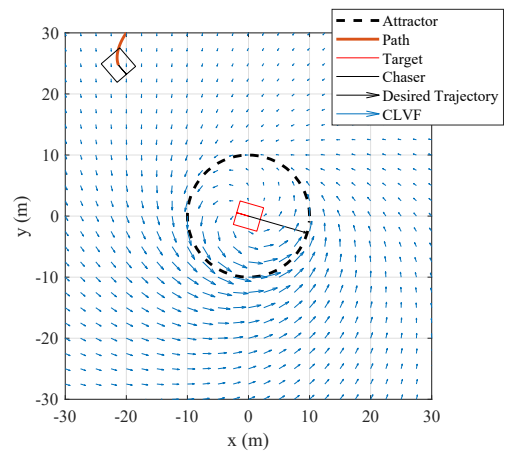

(b) $\mathrm{t}=28.57 \mathrm{~s}$.

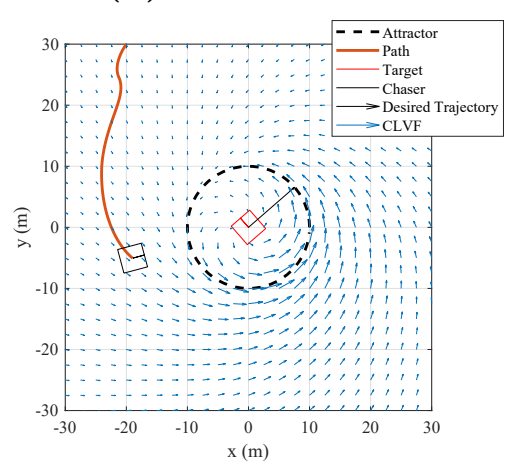

(d) $\mathrm{t}=85.71 \mathrm{~s}$.

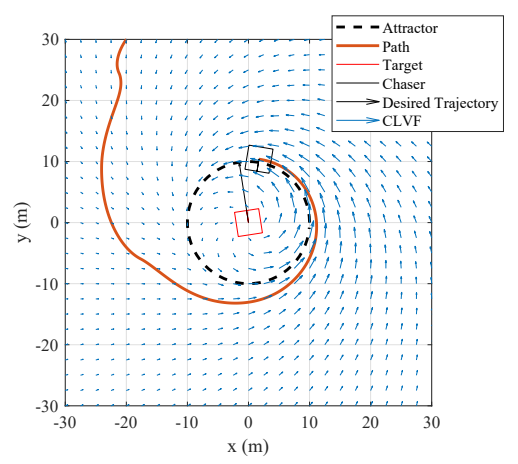

(f) $\mathrm{t}=142.86 \mathrm{~s}$.

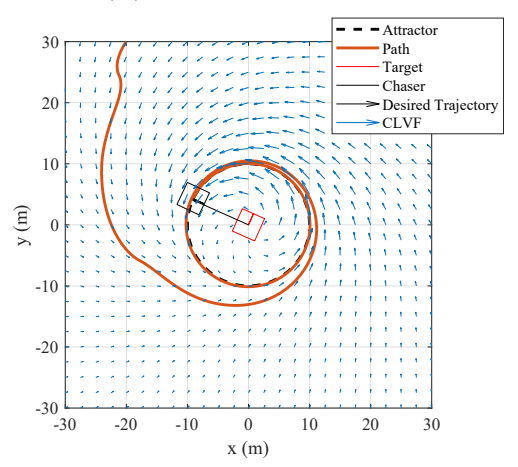

(h) $\mathrm{t}=200.00 \mathrm{~s}$.

Figure 3.11: Time-lapse of $u_{\max }=0.7 \mathrm{~m} / \mathrm{s}^{2}$ - case 1 . 
possible that in some design cases, there may be some small set of worst case scenarios with quite high acceleration commands, but yet, a very low likelihood of occurring. In these particular cases, the current technique of finding an absolute bound results in an "over-designed" field which very infrequently uses the full acceleration capabilities. Of course, an improved parameter selection procedure could likely circumvent this issue, and a few methods which could improve the parameter selection are breifly discussed below.

One approach to parameter selection could involve statistical analysis rather than an absolute upper bound. That is, a bound can be selected where there is some estimated (high) probability that the vehicle will not be commanded above the acceleration limits. While this method may increase the computation to select parameters, the important thing is that these parameters are only selected once. Afterwards, the controlled vehicle is able to meet acceleration and path constraints simply by following the designed guidance and control laws without any use of computationally heavy closed-loop algorithms. Yet another alternative method could be to use adaptive field parameters to better exploit the full acceleration capabilities. While this would increase the computational effort of running the CLVF online, the increased computation of running some adaptive parameters is quite low compared to the computational power required for in-the-loop optimization algorithms such as model predictive control.

An alternative approach to the parameter optimization (which will be explored further in Chapter 5) is to estimate the maneuver time and fuel expenditure as a function of the field parameters. In this case, the field parameters could be selected according to an optimization of the following form

$$
\begin{aligned}
\min _{b, k_{c}, k_{a}} & W_{f} \hat{f}\left(b, k_{c}, k_{a}\right)+W_{t} \hat{t}\left(b, k_{c}, k_{a}\right) \\
\text { subject to } & b, k_{c}, k_{a}>0 \\
& u_{\max } \geq u_{\mathrm{CLVF}}\left(b, k_{c}, k_{a}\right)
\end{aligned}
$$

where $\hat{f}$ and $\hat{t}$ are "estimates" of the fuel expenditure and time to converge to the desired trajectory, $W_{i}$ are user-selected weights, and $u_{\mathrm{CLVF}}\left(b, k_{c}, k_{a}\right)$ represents the 
right-hand-side of the acceleration constraint inequality (in this Chapter, Eq. (3.123)) to enforce the acceleration constraint.

\subsection{Conclusions}

The development of cascaded Lyapunov vector fields has been presented to generalize applications of Lyapunov vector fields into scenarios where the desired trajectory is in a translating and rotating reference frame, and the overall trajectory is acceleration rather than velocity constrained.

A stability analysis showed globally asymptotic stability to the desired trajectory point provided C1)-C6) are met (or D1)-D3) for the simplified case). A robustness study using very common assumptions then showed that under convergence errors to the attractor, the stable and unstable equilibrium points are replaced by stable and unstable equilibrium "regions" of a small thickness. The size of these regions is dependent on the rate at which the stabilizing guidance grows compared to the rate at which the disturbance terms grow, which are controlled by the designer.

Particular study of a special cascaded Lyapunov vector field with broadly applied assumptions such as: a spherical attractor; a centered target, and; velocity components which align with the Lyapunov function gradients was performed, and sufficient conditions to guarantee an upper acceleration bound given by E1)-E3) were derived.

Furthermore, a particular design strategy for cascaded Lyapunov vector fields was developed, and an example of this design strategy was performed successfully for a spinning target spacecraft observation mission. These simulations showed that the combined cascaded Lyapunov vector field guidance and control was successfully able to satisfy the path and acceleration constraints under various different acceleration capabilities and initial conditions. Importantly, these constraints were met without the use of any computationally heavy in-the-loop algorithms. It is also noteworthy that examples which include time-dependent trajectories within the attractor have been presented. While such trajectories are not prohibited by the general Lyapunov vector field theory, the author is not aware of any cases where they have been used

previously, and the inclusion of a second alignment field provides an explicit method for including time-dependent trajectories. 
Some viable paths for improved parameter selection have also been briefly discussed, which may involve statistical analysis or adaptive parameters. Parameter selection which considers statistical fuel optimization or other performance measures should also be investigated. 


\section{Chapter 4}

\section{Docking Problem Formulation}

For the remainder of this work, the relative dynamics of two spacecraft in a similar orbit will be considered. One spacecraft, herein referred to as the "target", is assumed to be uncontrolled and tumbling about all three axes. The second spacecraft, herein referred to as the "chaser" is the controlled vehicle.

Before completing the CLVF-LVF docking maneuver design, it is necessary to introduce the framework within which the docking problem will be solved. First, the reference frames required for the remaining Chapters will be introduced in Sec. 4.1. Then, the necessary orbital dynamics and attitude dynamics equations are introduced in Sec. 4.2. Section 4.3 introduces the multi-phase approach to the designed docking maneuver, and the vectors used to define the target spacecraft layout are outlined in Sec. 4.4. Finally, the structure of the new docking system, including a high-level block diagram is given in Sec. 4.5 .

\subsection{Reference Frame Definitions}

In the following, Sec. 4.1.1 introduces the Earth-centered inertial (ECI) reference frame, and Sec. 4.1.2 introduces the body-fixed reference frame.

\subsubsection{Earth-Centered Inertial (ECI) Reference Frame}

As the name implies, ECI has an origin at the Earth's center and orientation which is fixed with reference to the stars. The ECI $x$ vector $\boldsymbol{I}_{x}$ points in the direction of Aries, $\boldsymbol{I}_{z}$ points in the direction of Earth's spin axis, and $\boldsymbol{I}_{y}$ is defined by $\boldsymbol{I}_{y}=\boldsymbol{I}_{z}^{\times} \boldsymbol{I}_{x}$ to complete the right-handed triad. The ECI frame will be a very natural selection when simulating the orbital mechanics of both satellites. This reference frame is illustrated in Fig. 4.1 with a target spacecraft at position $\boldsymbol{R}_{t} \in \mathbb{R}^{3}$ and a chaser spacecraft at 
position $\boldsymbol{R}_{c} \in \mathbb{R}^{3}$. The relative position vector $\boldsymbol{r} \in \mathbb{R}^{3}$ is also shown, defined as $\boldsymbol{r}=\boldsymbol{R}_{c}-\boldsymbol{R}_{t}$

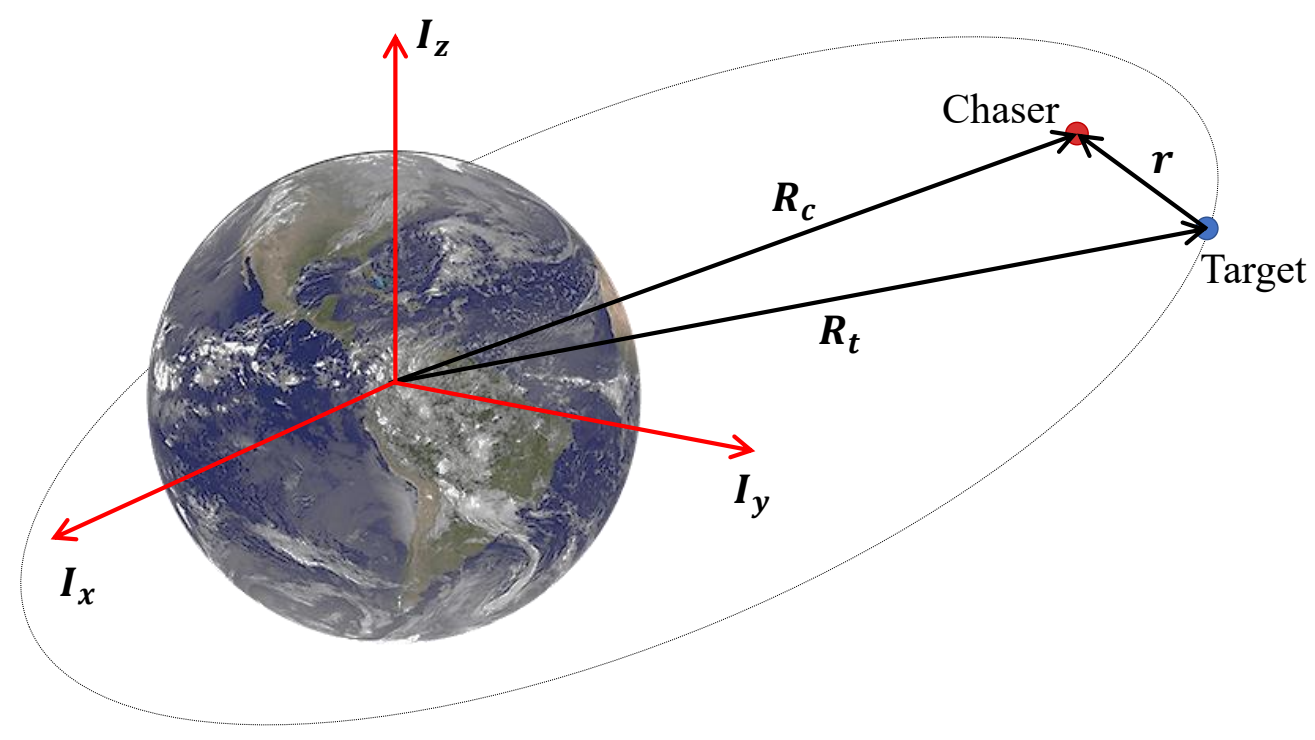

Figure 4.1: Definition of Earth-centered inertial reference frame.

\subsubsection{Body-Fixed Reference Frame}

Many vectors throughout this work remain constant to an observer who is fixed to the tumbling target spacecraft. Therefore, it is convenient to define a body-fixed reference frame with an origin at the target spacecraft's center of mass and an orientation which is fixed to that of the target spacecraft. Without loss of generality, it can be assumed that the axes of this reference frame align with the principal inertial axes of the target [29]. The body-fixed reference frame is useful when describing the attitude dynamics of the tumbling target.

When coordinates must be expressed in this reference frame, the vector will be given a superscript $B$.

\subsection{Spacecraft Dynamics}

The dynamics of the spacecraft will generally be simulated as independent translation and attitude systems. The translation dynamics of each spacecraft will evolve 
according to orbital dynamics equations, whereas the attitude dynamics will evolve according to Euler's rotational dynamics.

\subsubsection{ECI Orbital Dynamics}

In general, the orbits considered in this work will be elliptical with perturbations from $J_{2}$ (a perturbation term which arises from the oblateness of the Earth). The perturbed inertial dynamics of each vehicle are then given by [30]

$$
\ddot{\boldsymbol{R}}_{i}=-\mu \frac{\boldsymbol{R}_{i}}{R_{i}^{3}}+\frac{3 \mu J_{2} E_{R}^{2}}{2 R_{i}^{5}}\left\{\left(\frac{5 R_{z, i}^{2}}{R_{i}^{2}}-1\right) \boldsymbol{R}_{i}-2 R_{z, i} \boldsymbol{I}_{z}\right\}+\boldsymbol{u}_{i}
$$

where $i$ can be either $c$ for the chaser or $t$ for the target, $\mu \in \mathbb{R}$ is the gravitational parameter of Earth, $E_{R} \in \mathbb{R}$ is the Earth's radius, $J_{2} \in \mathbb{R}$ is the earth oblateness perturbation constant, $\boldsymbol{u}_{i} \in \mathbb{R}^{3}$ is the acceleration control input (note that $\boldsymbol{u}_{t}=\mathbf{0}$ ), $\boldsymbol{R}_{i}$ is the position vector of the chosen spacecraft with ECI coordinates

$$
\boldsymbol{R}_{i}=\left[\begin{array}{lll}
R_{x, i} & R_{y, i} & R_{z, i}
\end{array}\right]^{T}
$$

and

$$
R_{i}=\left\|\boldsymbol{R}_{i}\right\|
$$

Note that, while Eq. (4.1) is used to propagate the translational dynamics of each spacecraft, it is often easiest to define initial conditions in terms of classical orbital elements. The six classical orbital elements which will be converted into initial Cartesian ECI position and velocity vectors are:

- Semi-major axis $\left(œ_{a}\right)$ which is given in $\mathrm{km}$. Determines the orbit size.

- Eccentricity $\left(œ_{e}\right)$ which is dimensionless. Determines how elliptical the orbit is. For this work, only values in the range $0 \leq œ_{e}<1$ will be considered. An orbit with $œ_{e}=0$ is perfectly circular, and the orbit becomes increasingly elliptical as $œ_{e} \rightarrow 1$. Note that $œ_{e} \geq 1$ corresponds to orbits that are not closed (i.e. the orbiting object escapes the gravity field of the primary body). In particular, an orbit with $o e_{e}=1$ is parabolic, whereas orbits with $o e_{e}>1$ are hyperbolic. 
- Inclination $\left(œ_{i}\right)$ which is given in radians. Determines the angle between $\boldsymbol{I}_{z}$ and the orbit normal vector.

- Right Ascension of the Ascending Node $\left(œ_{\Omega}\right)$ which is given in radians. Determines the location where the orbit crosses from the southern hemisphere into the northern hemisphere (i.e., the ascending node), as measured by a positive angle about the $\boldsymbol{I}_{z}$ axis, where $\boldsymbol{I}_{x}$ is the reference 0 angle.

- Argument of Perigee $\left(œ_{\omega}\right)$ which is given in radians. Gives the angle from the ascending node to the orbit perigee within the orbital plane.

- True Anomaly $\left(\infty_{\theta}\right)$ which is given in radians. Determines the location of the spacecraft relative to the perigee within the orbital plane.

Procedures to convert initial orbital elements into initial Cartesian conditions $\boldsymbol{R}_{i}$ and $\dot{\boldsymbol{R}}_{i}$ are available in Appendix B.

Only when developing the Lyapunov vector field method, it will be assumed that both spacecraft are in similar orbits and that the maneuver dynamics are much faster than the orbital dynamics, and therefore the acceleration difference due to differences in gravity are negligible compared to the thruster inputs [31]. That is

$$
\ddot{\boldsymbol{r}}=\boldsymbol{u}_{c}+\boldsymbol{p}_{g} \approx \boldsymbol{u}_{c}
$$

where $\boldsymbol{p}_{g} \in \mathbb{R}^{3}$ is a perturbation term from differences in gravity and $J_{2}$. Note that in simulations, the full nonlinear model with perturbations will be used.

\subsubsection{Body-Fixed Attitude Dynamics}

While the attitude of the chaser spacecraft is assumed to be well controlled, the attitude of the target spacecraft is assumed to be tumbling in an unforced environment. This motion therefore follows the well-known Euler's equation of attitude motion, given by

$$
\dot{\boldsymbol{\omega}}_{B I}=-\boldsymbol{J}^{-1}\left(\boldsymbol{\omega}_{B I}^{\times} \boldsymbol{J} \boldsymbol{\omega}_{B I}\right)
$$

where $\boldsymbol{J} \in \mathbb{R}^{3 \times 3}$ is the body-fixed inertia matrix of the target, and $\boldsymbol{\omega}_{B} I \in \mathbb{R}^{3}$ is the angular velocity vector of the target. Note that the mass matrix is always positive 
definite. The rotation matrix defining the target attitude evolves according to the following kinematics

$$
\dot{C}_{B I}=-\boldsymbol{\omega}_{B I}^{\times} \boldsymbol{C}_{B I}
$$

\subsection{Docking Phases}

The docking procedure is divided into one observation phase (Phase 0), and two maneuver phases (Phase 1 and Phase 2). During Phase 0, the chaser observes a few cycles of the target spacecraft tumbling motion to determine certain maximum characteristics (such as the maximum angular speed) or to fit an on-board dynamics model of the target. Note that this model-fitting concept is not used within the scope of the thesis. These maximum tumbling characteristics will then be used for the design of parameters in the subsequent phases, guaranteeing that the required acceleration is within the constraints defined by the chaser vehicle. During Phase 1, the chaser aims to align with the docking port orientation at a distance $\alpha \in \mathbb{R}>0$ from the target center of mass, and a distance $\alpha^{\prime} \in \mathbb{R}>0$ from the docking position. Then, during Phase 2, the two spacecraft are brought into contact. These three phases are visualized in Fig. 4.2.

Because Phase 2 occurs within a small radius $\alpha^{\prime}$ where the attitude must be tracked perfectly, a LVF built within the tumbling body-fixed reference frame is well suited for final contact. Phase 1, however, can start quite far from the target. Therefore, although conventional LVFs are ill-suited for this phase, the newly-developed CLVF is an adequate candidate solution to address this problem.

\subsection{Target and Chaser Spacecraft Vector Definitions}

Note that several definitions will be analogous for Phases 1 and 2. To highlight these similarities between Phases, it will be common in this thesis to define variables useful for Phase 2 with a prime superscript if they are analogous to measurements used for Phase 1. For example, there will be a useful angle measurement, $\theta$, in Phase 1 (defined below). For Phase 2, there is an analogous (but separate) angle measurement which will be defined as $\theta^{\prime}$. 


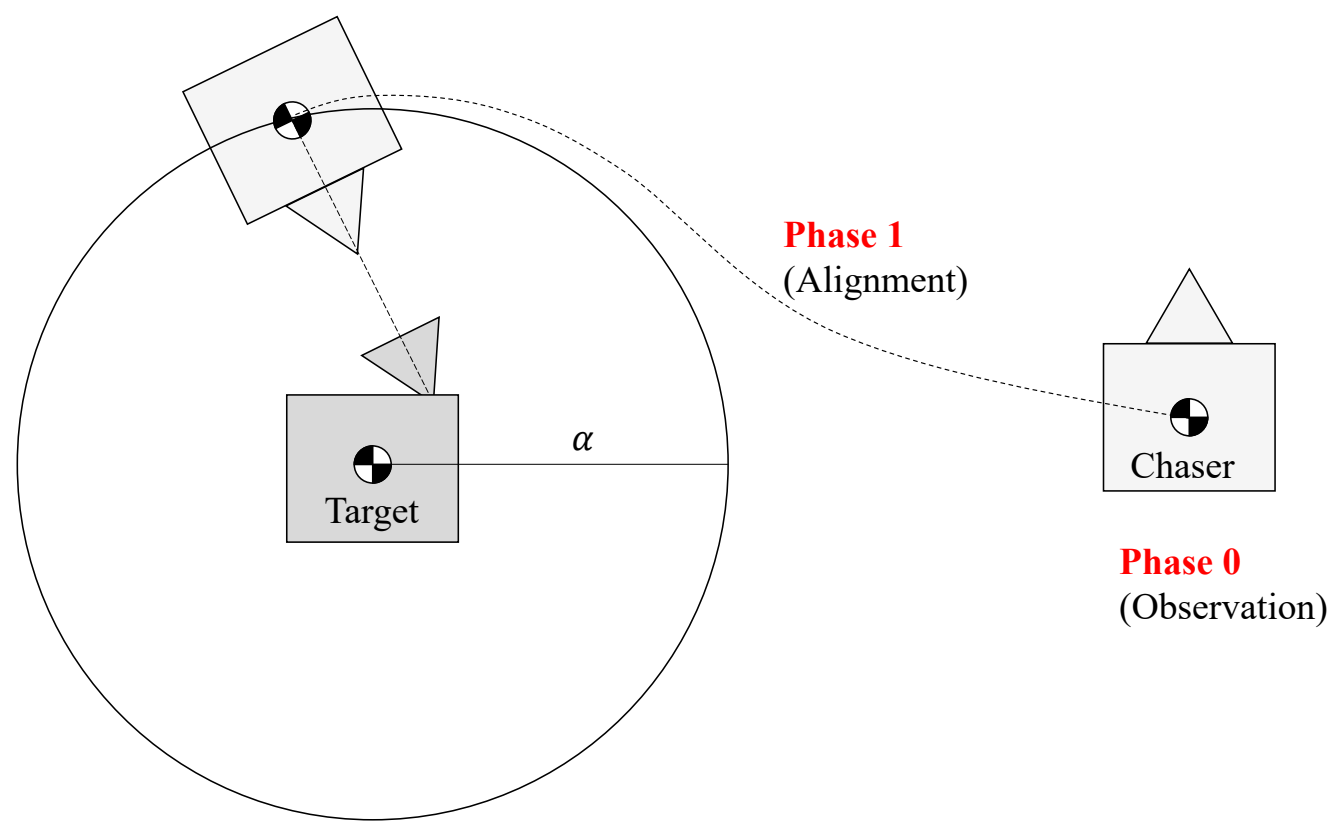

(a) Phases 0 and 1: observation and alignment.

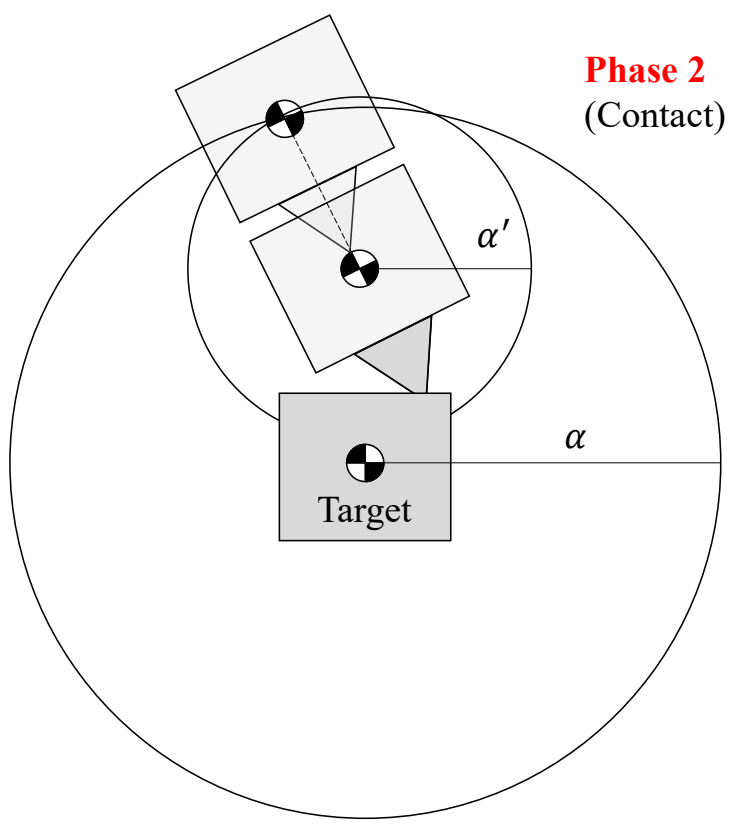

(b) Phase 2: contact.

Figure 4.2: Docking phases. 
The tumbling target has an angular rate of $\boldsymbol{\omega}_{B I}(t) \in \mathbb{R}^{3}$, and the required position for docking is at $\boldsymbol{d}(t) \in \mathbb{R}^{3}$. The orientation of the target docking port is defined by the unit vector $\hat{\boldsymbol{o}}^{\prime}(t) \in \mathbb{R}^{3}$. Note that the docking port is modelled as conic with an angle of acceptance given by $\theta_{d} \in(0, \pi / 2)$. The relative position of the chaser with respect to the docking position is given by $\boldsymbol{r}_{d}(t)=\boldsymbol{r}(t)-\boldsymbol{d}(t)$. In addition, the alignment angle defined between $\hat{\boldsymbol{o}}^{\prime}$ and $\boldsymbol{r}_{d}$ is defined as $\theta^{\prime} \in[0, \pi]$. A diagram of this setup is given in Fig. 4.3.

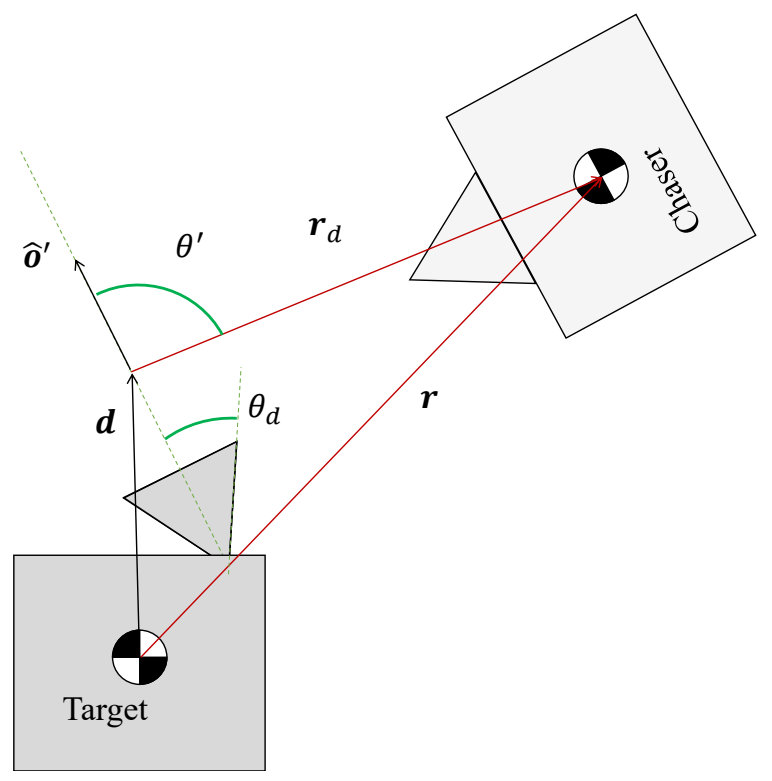

Figure 4.3: Target and chaser vector definitions.

For Phase 1, there are analogous (but separate) definitions for an alignment angle $\theta \in \mathbb{R}$ and an alignment vector $\hat{\boldsymbol{o}} \in \mathbb{R}^{3}$ for the position vector $\boldsymbol{r}$. The geometry relating $\boldsymbol{d}, \hat{\boldsymbol{o}}, \hat{\boldsymbol{o}}^{\prime}, \alpha$ and $\alpha^{\prime}$ is summarized in Fig. 4.4. 


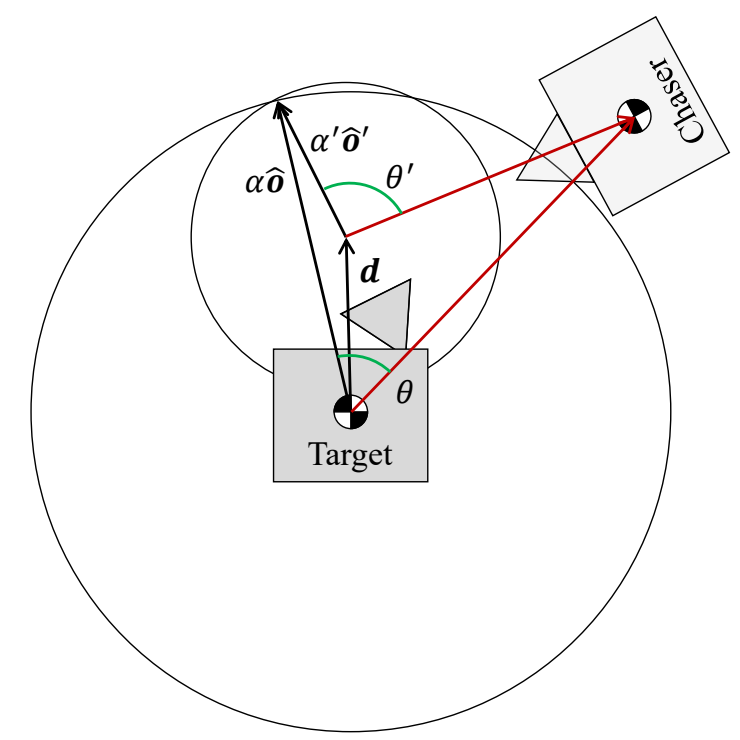

Figure 4.4: Alignment geometry for phases one and two.

As is visible from Fig. 4.4, the following geometric relationship exists

$$
\alpha \hat{\boldsymbol{o}}=\boldsymbol{d}+\alpha^{\prime} \hat{\boldsymbol{o}}^{\prime}
$$

\subsection{Feedback Structure}

A block diagram of the different components of the docking system is provided in Fig. 4.5. In the Phase 0 block, optimized vectors of parameters $\boldsymbol{p}_{P 1}$ and $\boldsymbol{p}_{P 2}$ are output to the CLVF and LVF blocks of Phases 1 and 2, respectively. Figure 4.6 shows the structure within the blocks for Phase 1 and 2. Subsequent Chapters will primarily develop and validate the guidance blocks and feed-forward acceleration blocks for Phase 1 and Phase 2. A particular parameter optimization strategy for Phase 0 will also be investigated. Note that in Fig. 4.6, the vector $\boldsymbol{p}_{P i}$ is used as a stand-in for either vector of optimized parameters. 


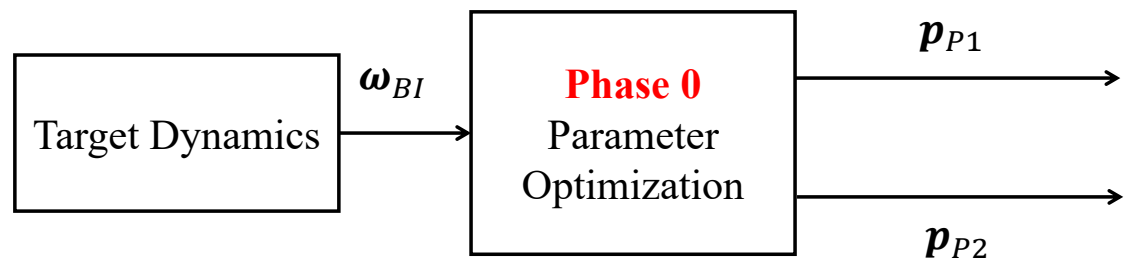

(a) Phase 0 diagram.

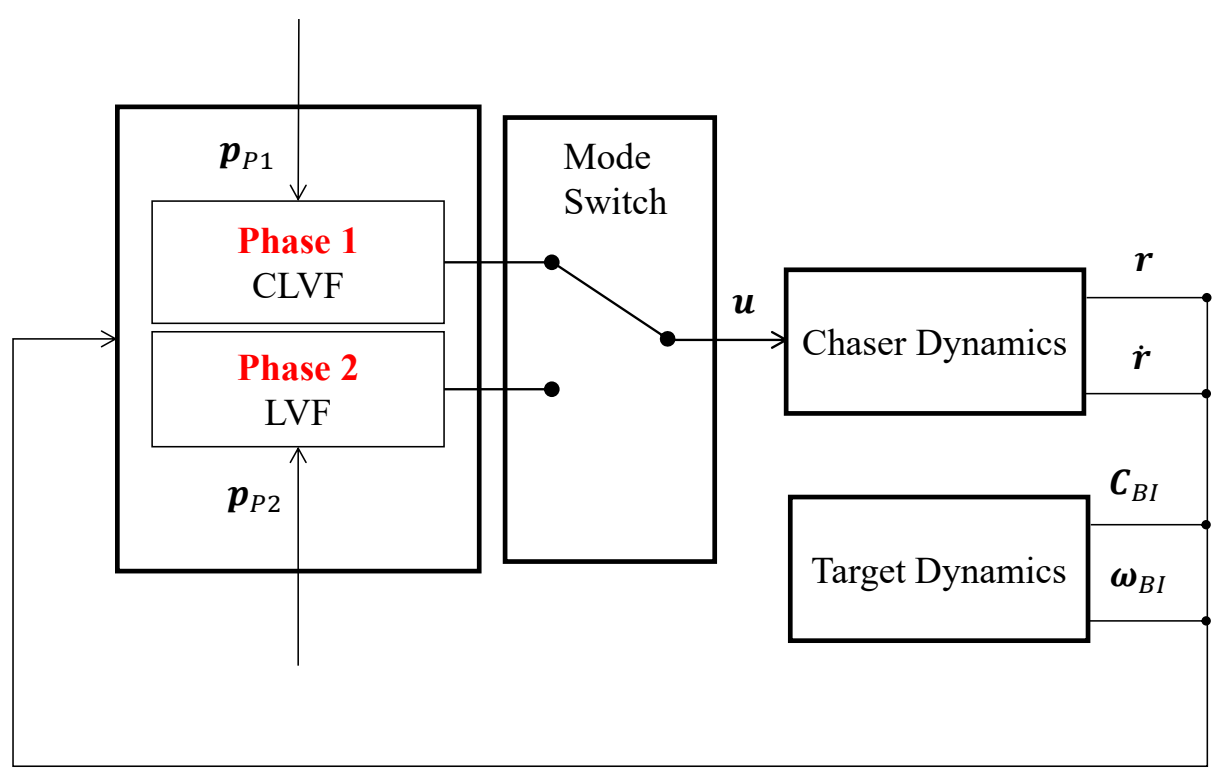

(b) Phases 1 and 2 switching diagram.

Figure 4.5: High level feedback structure. 


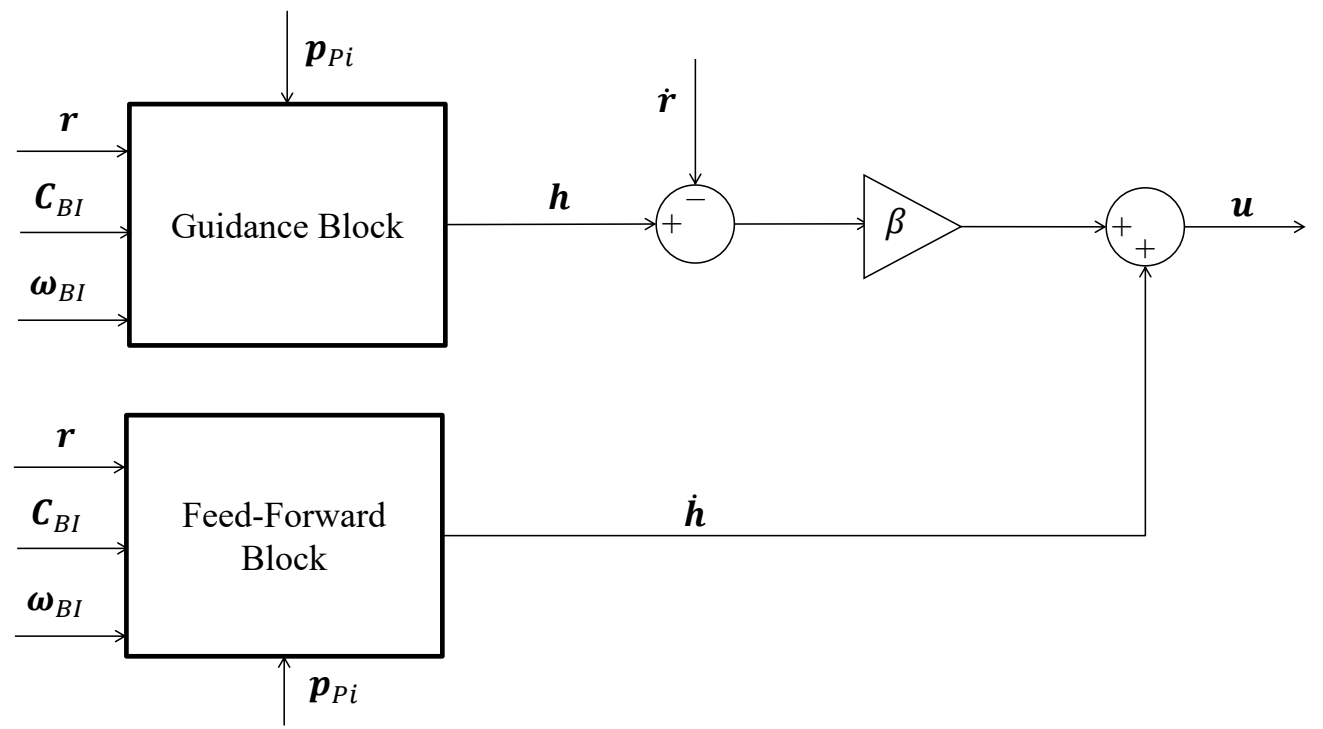

Figure 4.6: Feedback structure of LVF and CLVF systems. 


\section{Chapter 5}

\section{Docking Maneuver Design}

In this Chapter, the requirements for Phase 0 (the observation phase) are discussed in Sec. 5.1. The CLVF (Sec. 5.2) and LVF (Sec. 5.3) for Phases 1 and 2 respectively are then constructed, and their sets of feasible parameters which respect acceleration constraints are derived. It is proven that these parameters sets are convex. Finally, one particular maneuver time and fuel estimation technique is explored, and using these estimation functions a convex optimization process is developed in Sec. 5.4 for the parameter selection. A mode-switching criterion, although not the focus of this work, is briefly covered in Sec. 5.5.

\subsection{Phase 0 Design}

As will become clear throughout the upcoming design process, there are three target attitude dynamics norms which must be attained during the observation phase. These norms, denoted $n_{1}, n_{2}$, and $n_{3}$ are

$$
\begin{gathered}
n_{1}=\left\|\boldsymbol{\omega}_{B I}\right\|_{\max } \\
n_{2}=\left.\left\|\boldsymbol{\omega}_{B I}^{\times} \boldsymbol{\omega}_{B I}^{\times}+\dot{\boldsymbol{\omega}}_{B I}^{\times}\right\|\right|_{\max } \\
n_{3}=\left\|\left(\boldsymbol{\omega}_{B I}^{\times} \boldsymbol{\omega}_{B I}^{\times}+\dot{\boldsymbol{\omega}}_{B I}^{\times}\right) \boldsymbol{d}\right\|_{\max }
\end{gathered}
$$

Clearly, $n_{1}$ can be used to bound Coriolis accelerations, $n_{2}$ represents the total centripetal and tangential acceleration which is possible at a given radius from the target center, and $n_{3}$ represents the maximum acceleration required to hold the docking position.

These norms can either be obtained directly (i.e., through recording of several $\boldsymbol{\omega}_{B I}$ and $\dot{\boldsymbol{\omega}}_{B I}$ values over time), or, the target can be fit to a dynamics model using 
strategies such as those outlined in [32], and these norms can be derived through knowledge of that model. For the remainder of this work, it will be assumed that there is a valid method which attains and stores these norms in Phase 0 . It is assumed that these norms remain constant over time for the target attitude motion, which is a good assumption provided the maneuver takes place over a relatively short timescale. Over long timescales (say, the order of several orbit periods) secular disturbances will cause the attitude norms of an uncontrolled target to drift slowly [33]. In turn, the constant norms assumption would no longer be valid.

Although the parameter optimization is contained within Phase 0, it will not be covered until Sec. 5.4 after the specific design of Phase 1 and Phase 2 guidance have been completed.

\subsection{Phase 1 Design}

Below, the CLVF for Phase 1 is developed. First, the guidance law is selected according to the desired behaviour. Then, a convex set of feasible parameters is derived by use of Eq. (3.98).

\subsubsection{Guidance Law}

For the Phase 1 guidance, a simplified CLVF is chosen. The distance function $g(r)$ and the speed function $s_{a}(r, \theta)$ are selected identical to the design example given in Chapter 3.4. That is,

$$
\begin{gathered}
g(r)= \begin{cases}r, & r<\alpha \\
\frac{\alpha^{2}}{r}, & \text { otherwise. }\end{cases} \\
s_{a}(r, \theta)= \begin{cases}k_{a} \frac{r}{\alpha} \sin \theta, & r<\alpha \\
k_{a} \frac{\alpha}{r} \sin \theta, & \text { otherwise. }\end{cases}
\end{gathered}
$$


where $k_{a} \in \mathbb{R}>0$ is an unselected parameter. The contraction velocity function is selected as

$$
v_{c}(r)= \begin{cases}-\frac{k_{c}}{b^{2}}(r-\alpha)^{2}-2 \frac{k_{c}}{b}(r-\alpha), & 0<r-\alpha \leq b \\ \frac{k_{c}}{b^{2}}(r-\alpha)^{2}-2 \frac{k_{c}}{b}(r-\alpha), & -b \leq r-\alpha \leq 0 \\ k_{c} \operatorname{sgn}(\alpha-r), & \text { otherwise. }\end{cases}
$$

which $k_{c}$ and $b$ are unselected parameters. This function is selected for the favourable quality of a continuous derivative, as is illustrated in Fig. 5.1 for the specific case of $k_{c}=1$ and $b=4$.

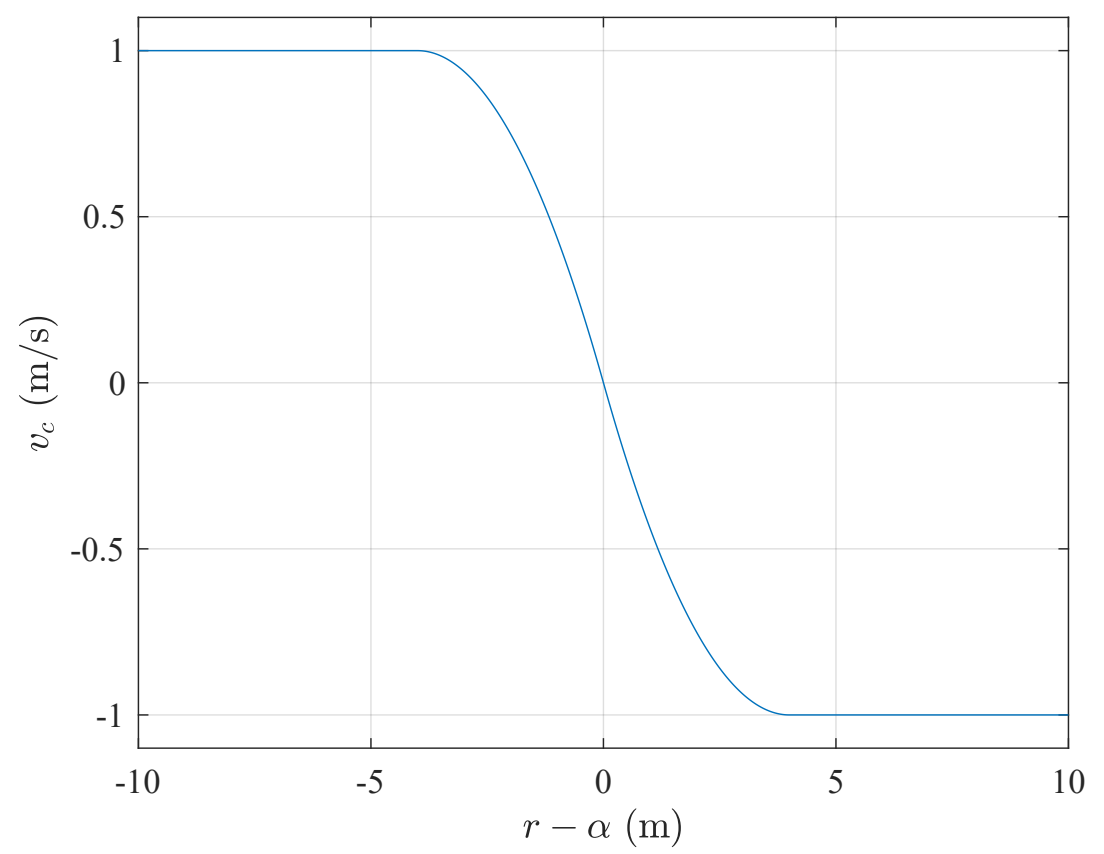

Figure 5.1: Contraction velocity function, $k_{c}=1, b=4$.

Clearly, the stability conditions D1)-D3) and the bounded acceleration conditions E1)-E3) are each met. The alignment angle $\theta$ and distance error $|r-\alpha|$ will therefore be driven asymptotically to zero with bounded acceleration. Because the docking problem is three-dimensional and the choice for $v_{c}(r)$ has changed, the acceleration bound from Chapter 3.4 cannot be reused, and a new set of feasible parameters is derived in the next subsection. 


\subsubsection{Upper Bound of Required Tracking Acceleration}

The following feed-forward acceleration occurs under perfect tracking, as seen from Eq. (3.98)

$$
\begin{aligned}
\left.\dot{\boldsymbol{h}}\left(\boldsymbol{r}, \hat{\boldsymbol{o}}, \boldsymbol{\omega}_{B I}, \dot{\boldsymbol{d}}\right)\right|_{\dot{\boldsymbol{r}}=\boldsymbol{h}}=\phi_{1}(r, \theta) \hat{\boldsymbol{r}}+\phi_{2}(r, \theta) \hat{\boldsymbol{a}} & +\boldsymbol{\omega}_{B I}^{\times} \boldsymbol{\Omega}_{1}(\boldsymbol{r}, \hat{\boldsymbol{o}})+\Omega_{2}\left(\boldsymbol{r}, \hat{\boldsymbol{o}}, \boldsymbol{\omega}_{B I}\right) \\
& +\frac{g^{2}(r, \theta)}{r} \boldsymbol{\omega}_{B I}^{\times} \boldsymbol{\omega}_{B I}^{\times} \hat{\boldsymbol{r}}+g(r, \theta) \dot{\boldsymbol{\omega}}_{B I}^{\times} \hat{\boldsymbol{r}}
\end{aligned}
$$

where

$$
\begin{gathered}
\phi_{1}(r, \theta)=\left.\dot{v}_{c}(r, \theta)\right|_{\dot{\boldsymbol{r}}=\boldsymbol{h}}-\frac{s_{a}^{2}(r, \theta)}{r} \\
\phi_{2}(r, \theta)=\left.\dot{s}_{a}(r, \theta)\right|_{\dot{\boldsymbol{r}}=\boldsymbol{h}}+\frac{s_{a}(r, \theta) v_{c}(r, \theta)}{r} \\
\boldsymbol{\Omega}_{1}(\boldsymbol{r}, \hat{\boldsymbol{o}})=\left(\left.\dot{g}(r, \theta)\right|_{\dot{\boldsymbol{r}}=\boldsymbol{h}}+\frac{g(r, \theta)}{r} v_{c}(r, \theta)\right) \hat{\boldsymbol{r}}+\left(s_{a}(r, \theta)+\frac{g(r, \theta)}{r} s_{a}(r, \theta)\right) \hat{\boldsymbol{a}} \\
\boldsymbol{\Omega}_{2}\left(\boldsymbol{r}, \hat{\boldsymbol{o}}, \boldsymbol{\omega}_{B I}\right)=s_{a}(r, \theta)\left(1-\frac{g(r, \theta)}{r}\right)\left(\hat{\boldsymbol{r}} \boldsymbol{\omega}_{B I}^{T} \hat{\boldsymbol{e}}-\frac{\cos \theta}{\sin \theta} \hat{\boldsymbol{e}} \boldsymbol{\omega}_{B I}^{T} \hat{\boldsymbol{a}}\right)
\end{gathered}
$$

where, from the selection of $s_{a}(r, \theta)$ and $g(r, \theta)$, the terms $\phi_{2}(r, \theta)$ and $\boldsymbol{\Omega}_{1}(\boldsymbol{r}, \hat{\boldsymbol{o}})$ simplify to the following when $r \geq \alpha$

$$
\begin{gathered}
\phi_{2}(r, \theta)=\left.\frac{\partial s_{a}}{\partial \theta} \dot{\theta}\right|_{\dot{\boldsymbol{r}}=\boldsymbol{h}} \\
\boldsymbol{\Omega}_{1}(\boldsymbol{r}, \hat{\boldsymbol{o}})=\left(s_{a}(r, \theta)+\frac{g(r, \theta)}{r} s_{a}(r, \theta)\right) \hat{\boldsymbol{a}}
\end{gathered}
$$

First, by observation of the tumbling motion (i.e., performing Phase 0), the final terms of Eq. (5.7) can be bounded by

$$
\left\|\frac{g^{2}(r, \theta)}{r} \boldsymbol{\omega}_{B I}^{\times} \boldsymbol{\omega}_{B I}^{\times} \hat{\boldsymbol{r}}+g(r, \theta) \dot{\boldsymbol{\omega}}_{B I}^{\times} \hat{\boldsymbol{r}}\right\| \leq n_{2} \alpha
$$

Similarly, the term including $\Omega_{1}$ can be bounded as follows

$$
\left\|\omega_{B I}^{\times} \Omega_{1}\right\| \leq 2 n_{1} k_{a}
$$


and $\boldsymbol{\Omega}_{2}$ can be bounded by substituting $s_{a}(r, \theta)$ and $g(r)$ as

$$
\left\|\boldsymbol{\Omega}_{2}\right\|=\left\|k_{a} \frac{\alpha}{r}\left(1-\frac{\alpha^{2}}{r^{2}}\right)\left(\sin \theta \hat{\boldsymbol{r}} \boldsymbol{\omega}_{B I}^{T} \hat{\boldsymbol{e}}-\cos \theta \hat{\boldsymbol{e}} \boldsymbol{\omega}_{B I}^{T} \hat{\boldsymbol{a}}\right)\right\|
$$

Noting that both $\boldsymbol{\omega}_{B I}^{T} \hat{\boldsymbol{e}}$ and $\boldsymbol{\omega}_{B I}^{T} \hat{\boldsymbol{a}}$ must be less than $n_{1}$ attained from Phase 0 , it can be shown that

$$
\left\|\sin \theta \hat{\boldsymbol{r}} \boldsymbol{\omega}_{B I}^{T} \hat{\boldsymbol{e}}-\cos \theta \hat{\boldsymbol{e}} \boldsymbol{\omega}_{B I}^{T} \hat{\boldsymbol{a}}\right\| \leq n_{1}
$$

Moreover, the term $\frac{\alpha}{r}\left(1-\frac{\alpha^{2}}{r^{2}}\right)$ is maximized at the value $r=\sqrt{3} \alpha$, and therefore

$$
\frac{\alpha}{r}\left(1-\frac{\alpha^{2}}{r^{2}}\right) \leq \frac{2}{3 \sqrt{3}} \approx 0.3849
$$

Combining these maximums, the following bound occurs

$$
|| \boldsymbol{\omega}_{B I}^{\times} \boldsymbol{\Omega}_{1}+\boldsymbol{\Omega}_{2} \| \leq 2.3849 k_{a} n_{1}
$$

What remains is to find bounds on the relative acceleration terms $\phi_{1}$ and $\phi_{2}$. Substitution of the functions $s_{a}, v_{c}$, and Eq. (3.86) into $\phi_{1}$ results in

$$
\phi_{1}(r, \theta)=\frac{d v_{c}}{d r} v_{c}-\frac{k_{a}^{2} \alpha \sin ^{2} \theta}{r^{3}}
$$

which, noting that for the particular $v_{c}(r)$ function selected

$$
\frac{d v_{c}}{d r} v_{c} \leq \frac{0.7698 k_{c}^{2}}{b}
$$

Eq. (5.20) can be bounded by

$$
\left|\phi_{1}(r, \theta)\right| \leq \frac{0.7698 k_{c}^{2}}{b}+\frac{k_{a}^{2}}{\alpha}
$$

Similarly, it is found that

$$
\left|\phi_{2}(r, \theta)\right| \leq \frac{k_{a}^{2}}{2 \alpha}+\frac{2}{3 \sqrt{3}} k_{a} n_{1}
$$


where the bound given by Eq. (5.18) was again utilized. Finally, given that $\phi_{1} \hat{\boldsymbol{r}}$ and $\phi_{2} \hat{\boldsymbol{a}}$ are always orthogonal

$$
\left\|\phi_{1} \hat{\boldsymbol{r}}+\phi_{2} \hat{\boldsymbol{a}}\right\| \leq \sqrt{\left(\frac{0.7698 k_{c}^{2}}{b}+\frac{k_{a}^{2}}{\alpha}\right)^{2}+\left(\frac{k_{a}^{2}}{2 \alpha}+\frac{2}{3 \sqrt{3}} k_{a} n_{1}\right)^{2}}
$$

Finally, each of the bounds above can be combined to state that for a vehicle with an acceleration constraint $u_{\max }$, parameters drawn from the following set will always form a feasible trajectory

$$
u_{\max } \geq \sqrt{\left(\frac{0.7698 k_{c}^{2}}{b}+\frac{k_{a}^{2}}{\alpha}\right)^{2}+\left(\frac{k_{a}^{2}}{2 \alpha}+\frac{2}{3 \sqrt{3}} k_{a} n_{1}\right)^{2}}+2.385 k_{a} n_{1}+n_{2} \alpha
$$

Note that $\alpha$ must meet the geometric condition given by Eq. (4.7).

Theorem 5. The set of all $\left(\alpha, b, k_{a}, k_{c}\right)$ which meet Eq. (5.25) form a convex set, given that $\alpha>0, b>0, k_{a}>0$, and $k_{c}>0$.

Proof. It is first noted that the inequality Eq. (5.25) states that the right-hand side is bounded from above by a constant. Therefore, if the right-hand side of Eq. (5.25) is a convex function, then Eq. (5.25) is a convex set [34].

The last two terms given by $2.385 k_{a} n_{1}$ and $n_{2} \alpha$ are affine, and are therefore convex functions. Therefore, if the function

$$
q\left(\alpha, b, k_{a}, k_{c}\right)=\sqrt{\left(\frac{0.7698 k_{c}^{2}}{b}+\frac{k_{a}^{2}}{\alpha}\right)^{2}+\left(\frac{k_{a}^{2}}{2 \alpha}+\frac{2}{3 \sqrt{3}} k_{a} n_{1}\right)^{2}}
$$

is convex, then Eq. (5.25) will form a convex set. By defining

$$
\begin{aligned}
& q_{1}\left(\alpha, b, k_{a}, k_{c}\right)=\frac{0.7698 k_{c}^{2}}{b}+\frac{k_{a}^{2}}{\alpha} \\
& q_{2}\left(\alpha, b, k_{a}, k_{c}\right)=\frac{k_{a}^{2}}{2 \alpha}+\frac{2}{3 \sqrt{3}} k_{a} n_{1}
\end{aligned}
$$

the function $q$ can be reformulated as

$$
q\left(\alpha, b, k_{a}, k_{c}\right)=\sqrt{\left(q_{1}\left(\alpha, b, k_{a}, k_{c}\right)\right)^{2}+\left(q_{2}\left(\alpha, b, k_{a}, k_{c}\right)\right)^{2}}
$$


where clearly, $q$ is convex in $\left(q_{1}, q_{2}\right)$ and is strictly increasing in both $q_{1}$ and $q_{2}$. Therefore, if $q_{1}$ and $q_{2}$ are convex in $\left(\alpha, b, k_{a}, k_{c}\right)$, then $q$ is also convex in $\left(\alpha, b, k_{a}, k_{c}\right)$ by convex function composition rules. Therefore, convexity of the set given by Eq. (5.25) can be proven if the Hessians of $q_{1}$ and $q_{2}$ with respect to $\left(\alpha, b, k_{a}, k_{c}\right)$ are positive semi-definite, which is trivial to show under the conditions that $\alpha, b, k_{a}$ and $k_{c}$ are all strictly positive.

\subsection{Phase 2 Design}

Below, the LVF for Phase 2 guidance is developed. Recall that in Phase 2, the controlled spacecraft is in close proximity to the target. Therefore, the exact attitude of the target can be tracked without thruster saturation, and a classic LVF defined in the body-fixed frame is suitable. First, the guidance law is selected according to the desired behaviour. Then, a convex set of feasible parameters is derived using Eq. (3.98) with the special case of a distance function which equals the true distance.

\subsubsection{Guidance Law}

This Section performs a classic LVF construction. Therefore, to begin a Lyapunov function must be selected. Because it is desirable that the attractor $\mathbb{A}$ be the set $\left\{\boldsymbol{r}_{d}=\mathbf{0}\right\}$, the Lyapunov function $V_{P 2}$ is selected as

$$
V_{P 2}\left(r_{d}\right)=\frac{r_{d}^{2}}{2}
$$

which obviously meets conditions A1) - A4), where the controlled state is the vector $\boldsymbol{r}_{d}$. To ease the developments that follow, a normalized angle measurement is introduced

$$
\theta_{N}= \begin{cases}\frac{\theta^{\prime} \pi}{2 \theta_{d}}, & \text { for } \theta^{\prime} \leq \theta_{d} \\ \frac{\pi}{2}, & \text { otherwise. }\end{cases}
$$

At this point, the new body-fixed docking field can be defined with the structure

$$
\boldsymbol{h}^{B}=v\left(r_{d}, \theta^{\prime}\right)\left(\hat{\boldsymbol{c}}^{\prime B} \cos \theta_{N}+\hat{\boldsymbol{a}}^{\prime B} \sin \theta_{N}\right)
$$


where $v\left(r_{d}, \theta^{\prime}\right)$ is a speed function, and $\hat{\boldsymbol{c}}^{\prime} B$ and $\hat{\boldsymbol{a}}^{\prime B}$ are contraction and alignment direction vectors respectively, given by

$$
\begin{gathered}
\hat{\boldsymbol{c}}^{\prime B}=\boldsymbol{\Gamma}\left(\boldsymbol{r}_{d}^{B}\right){\frac{\partial V_{P 2}^{T}}{\partial \boldsymbol{r}_{d}^{B}}}^{\hat{\boldsymbol{a}}^{\prime}}=\frac{\hat{\boldsymbol{o}}^{\prime}+\hat{\boldsymbol{c}}^{\prime} \cos \theta}{\sin \theta}
\end{gathered}
$$

where

$$
\boldsymbol{\Gamma}\left(\boldsymbol{r}_{d}^{B}\right)=\frac{1}{\left\|\partial V_{P 2} / \partial \boldsymbol{r}_{d}^{B}\right\|} \boldsymbol{I}
$$

Note that if $\theta^{\prime} \leq \theta_{d}$ and $v\left(r_{d}, \theta^{\prime}\right)$ is strictly positive everywhere except the point $r_{d}=0$, then Eq. (5.32) meets B1) and B2) respectively, and therefore asymptotic stability to the point $\boldsymbol{r}_{d}=\mathbf{0}$ is achieved in this region.

Further, by considering Eqs. (5.31) and (5.32), it can be seen that the contraction component of velocity is zero until $\theta^{\prime}<\theta_{d}$. This is quite desirable, as contraction will not occur unless the two spacecraft are sufficiently aligned. Indeed, this represents one of the path constraints which can be inherently included in the guidance, rather than considered as a constraint in an optimizer. Also, when $\theta^{\prime}=0$ the alignment component of velocity is zero and the chaser spacecraft purely contracts to the docking port.

To bound the required acceleration, let us first solve for the equivalent Phase 2 guidance as seen from the inertial frame,

$$
\boldsymbol{h}=v\left(r_{d}, \theta^{\prime}\right)\left(\hat{\boldsymbol{c}} \cos \theta_{N}+\hat{\boldsymbol{a}} \sin \theta_{N}\right)+\boldsymbol{\omega}_{B I}^{\times}\left(\boldsymbol{r}_{d}+\boldsymbol{d}\right)
$$

It is easy to see that $\hat{\boldsymbol{c}}^{\prime}=-\hat{\boldsymbol{r}}_{d}$, and therefore Eq. (5.36) can be put into the familiar form

$$
\boldsymbol{h}=v_{c}^{\prime}\left(r_{d}, \theta^{\prime}\right) \hat{\boldsymbol{r}}_{d}+s_{a}^{\prime}\left(r_{d}, \theta^{\prime}\right) \hat{\boldsymbol{a}}+\boldsymbol{\omega}_{B I}^{\times} \boldsymbol{r}_{d}+\boldsymbol{\omega}_{B I}^{\times} \boldsymbol{d}
$$

which, of course, matches the simplified CLVF structure for a special case where $g\left(r_{d}, \theta^{\prime}\right)=r_{d}$ and $\dot{\boldsymbol{d}}=\boldsymbol{\omega}_{B I}^{\times} \boldsymbol{d}$, with speed functions

$$
v_{c}^{\prime}\left(r_{d}, \theta^{\prime}\right)=-v\left(r_{d}, \theta^{\prime}\right) \cos \theta_{N}
$$




$$
s_{a}^{\prime}\left(r_{d}, \theta^{\prime}\right)=v\left(r_{d}, \theta^{\prime}\right) \sin \theta_{N}
$$

That is, a LVF applied within the body-fixed frame can also be characterized as a CLVF which has already converged to the body-fixed frame.

What is left is to select a function for $v\left(r_{d}, \theta^{\prime}\right)$ according to the desired behaviour. For a smooth transition between phases, the function $v\left(r_{d}, \theta^{\prime}\right)$ should be near 0 when $r_{d}=\alpha^{\prime}$ Note that the function $v\left(r_{d}, \theta^{\prime}\right)$ cannot equal exactly to 0 except at $r_{d}=0$ to satisfy stability conditions. One simple selection for $v\left(r_{d}, \theta^{\prime}\right)$ is

$$
v\left(r_{d}\right)=v_{\max } \sin r_{N}
$$

where $v_{\max } \in \mathbb{R}>0$ is constant maximum relative speed, and $r_{N}$ is a normalized radius defined as

$$
r_{N}= \begin{cases}f \pi \frac{r_{d}}{\alpha^{\prime}}, & r_{d} \leq \alpha^{\prime} \\ f \pi, & \text { otherwise. }\end{cases}
$$

where $f \in(0,1)$, but is typically quite close to 1 . That is, the speed function is nearly zero when $r_{d}=\alpha^{\prime}$, but remains positive. As the chaser converges towards the target, the speed increases to a maximum before before decaying back to zero.

A visualization of the designed Phase 2 LVF is given in Fig. 5.2 in the target bodyfixed frame. Note that although the Phase 2 guidance does not require fundamentally new theory from previous LVF literature (i.e., it is fully built from A1)-A4) and B1)-B3)), the application and "shape" of the field is substantially different from previous work designed for UAV standoff tracking. There is also significant robustness to initialization errors inherent to the new field. For example, Fig. 5.3 shows one particular path which may occur if Phase 2 were initialized with very significant tracking error.

\subsubsection{Upper Bound of Required Tracking Acceleration}

Note that due to the structure of Eq. (5.37), the feed-forward acceleration of Eq. (3.98) can be used directly under the special case of $g\left(r_{d}, \theta^{\prime}\right)=r_{d}$. Therefore, the 


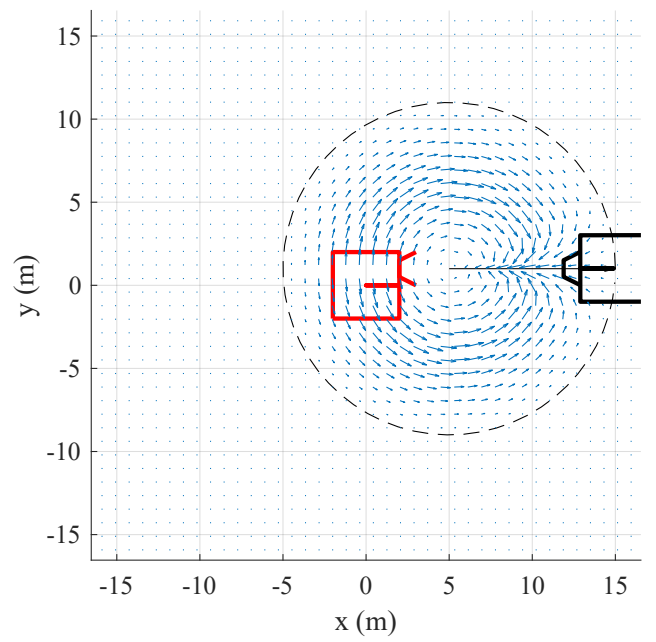

(a) Start of Phase 2.

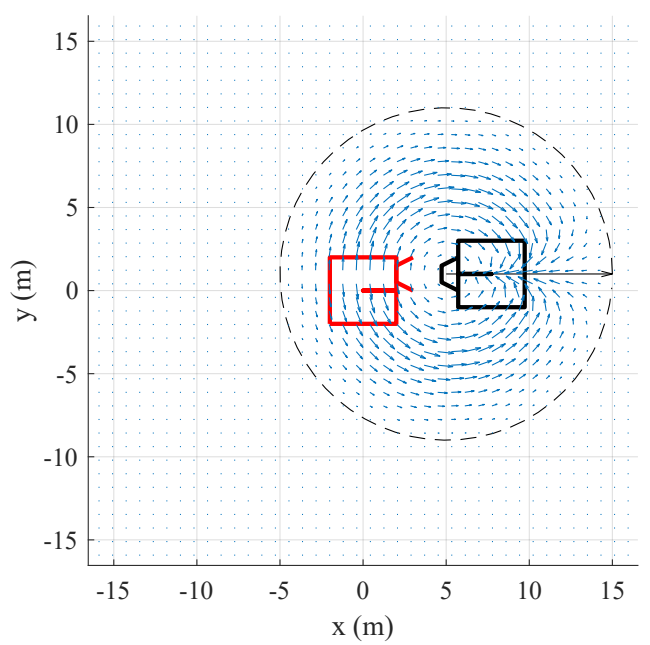

(b) Middle of Phase 2 .

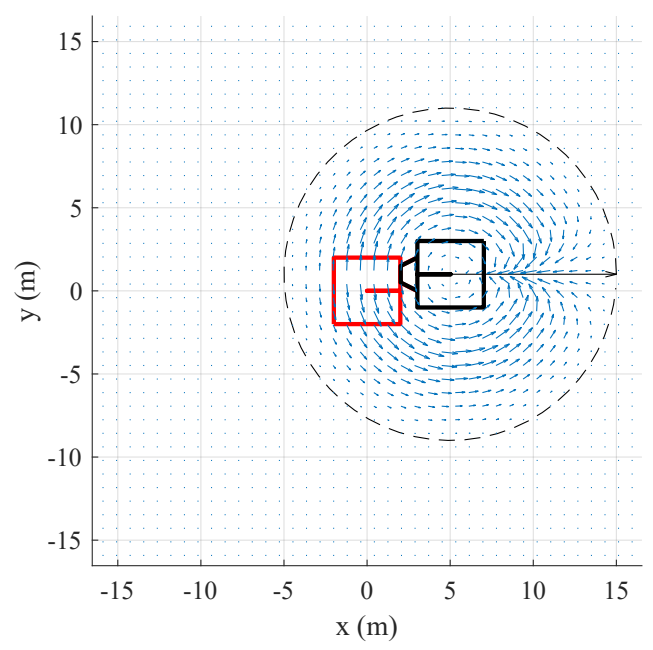

(c) End of Phase 2.

Figure 5.2: Illustration of Phase 2 LVF guidance under nominal conditions. 


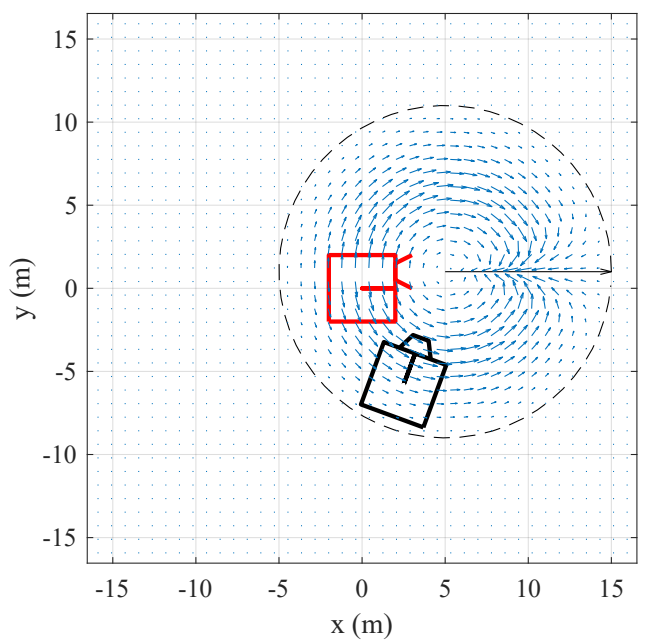

(a) Start of Phase 2.

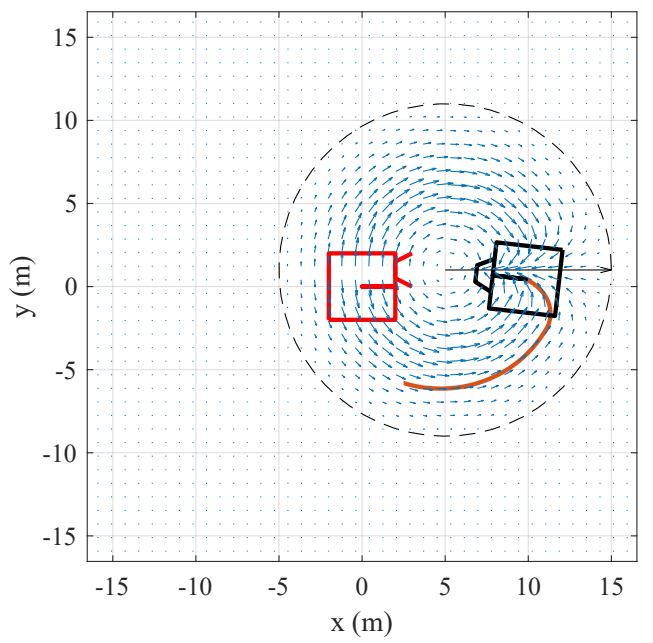

(b) Middle of Phase 2.

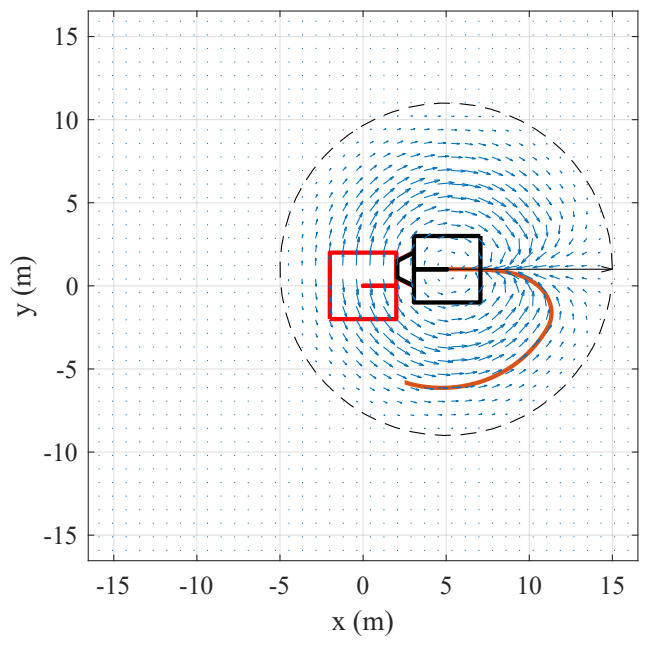

(c) End of Phase 2.

Figure 5.3: Illustration of Phase 2 LVF guidance initialized with significant tracking error. 
following feed-forward acceleration occurs under perfect tracking

$$
\begin{array}{r}
\left.\dot{\boldsymbol{h}}\left(\boldsymbol{r}_{d}, \hat{\boldsymbol{o}}^{\prime}, \boldsymbol{\omega}_{B I}, \dot{\boldsymbol{d}}\right)\right|_{\dot{\boldsymbol{r}}_{d}=\boldsymbol{h}}=\phi_{1}^{\prime}\left(r_{d}, \theta^{\prime}\right) \hat{\boldsymbol{r}}_{d}+\phi_{2}^{\prime}\left(r_{d}, \theta^{\prime}\right) \hat{\boldsymbol{a}}^{\prime}+2 \boldsymbol{\omega}_{B I}^{\times}\left(v_{c}^{\prime}\left(r_{d}, \theta^{\prime}\right) \boldsymbol{r}_{d}+s_{a}^{\prime}\left(r_{d}, \theta^{\prime}\right) \hat{\boldsymbol{a}}^{\prime}\right)+ \\
\boldsymbol{\omega}_{B I}^{\times} \boldsymbol{\omega}_{B I}^{\times}\left(\boldsymbol{r}_{d}+\boldsymbol{d}\right)+\dot{\boldsymbol{\omega}}_{B I}^{\times}\left(\boldsymbol{r}_{d}+\boldsymbol{d}\right)
\end{array}
$$

where

$$
\begin{gathered}
\phi_{1}^{\prime}\left(r_{d}, \theta^{\prime}\right)=\left.\dot{v}_{c}^{\prime}\left(r_{d}, \theta^{\prime}\right)\right|_{\dot{\boldsymbol{r}}=\boldsymbol{h}}-\frac{s_{a}^{2}\left(r_{d}, \theta^{\prime}\right)}{r_{d}} \\
\phi_{2}^{\prime}\left(r_{d}, \theta^{\prime}\right)=\left.\dot{s}_{a}^{\prime}\left(r_{d}, \theta^{\prime}\right)\right|_{\dot{\boldsymbol{r}}=\boldsymbol{h}}+\frac{s_{a}^{\prime}\left(r_{d}, \theta^{\prime}\right) v_{c}^{\prime}\left(r_{d}, \theta^{\prime}\right)}{r_{d}}
\end{gathered}
$$

which can be bounded in norm by

$$
\|\dot{\boldsymbol{h}}\|_{\dot{\boldsymbol{r}}=\boldsymbol{h}} \leq\left\|\phi_{1}^{\prime}\left(r_{d}, \theta^{\prime}\right) \hat{\boldsymbol{r}}_{d}+\phi_{2}^{\prime}\left(r_{d}, \theta^{\prime}\right) \hat{\boldsymbol{a}}^{\prime}\right\|+2 n_{1} v\left(r_{d}, \theta^{\prime}\right)+n_{3}+n_{2} r_{d}
$$

Applying the chain rule to Eq. (5.38) and Eq. (5.39)

$$
\begin{aligned}
\dot{v}_{c}^{\prime}\left(r_{d}, \theta^{\prime}\right) & =-\dot{v}\left(r_{d}\right) \cos \theta_{N}+v\left(r_{d}\right) \sin \theta_{N} \dot{\theta}_{N} \\
& =-\dot{v}\left(r_{d}\right) \cos \theta_{N}+s_{a}\left(r_{d}, \theta^{\prime}\right) \dot{\theta}_{N} \\
\dot{s}_{a}^{\prime}\left(r_{d}, \theta^{\prime}\right) & =\dot{v}\left(r_{d}\right) \sin \theta_{N}+v\left(r_{d}\right) \cos \theta_{N} \dot{\theta}_{N} \\
& =\dot{v}\left(r_{d}\right) \sin \theta_{N}-v_{c}\left(r_{d}, \theta^{\prime}\right) \dot{\theta}_{N}
\end{aligned}
$$

where, within the docking cone

$$
\left.\dot{\theta}_{N}\right|_{\dot{\boldsymbol{r}}=\boldsymbol{h}}=\frac{-\pi}{2 \theta_{d}} \frac{s_{a}\left(r_{d}, \theta^{\prime}\right)}{r_{d}}
$$

Substitution of Eq.'s (5.50), (5.48) and (5.49) into (5.43) and (5.44) then yields (after some rearranging)

$$
\left\|\phi_{1}^{\prime} \hat{\boldsymbol{r}}_{d}+\phi_{2}^{\prime} \hat{\boldsymbol{a}}^{\prime}\right\|^{2}=\left(\frac{d v}{d r_{d}} v\left(r_{d}\right)\right)^{2} \cos ^{2} \theta_{N}+\left(\left[1+\frac{\pi}{2 \theta_{d}}\right] \frac{v^{2}\left(r_{d}\right)}{r_{d}}\right)^{2} \sin ^{2} \theta_{N}
$$


Note that this is of the form

$$
\left\|\phi_{1}^{\prime} \hat{\boldsymbol{r}}_{d}+\phi_{2}^{\prime} \hat{\boldsymbol{a}}^{\prime}\right\|^{2}=a_{1} \zeta+a_{2}(1-\zeta)
$$

where $a_{1}$ and $a_{2}$ are positive real numbers and $\zeta \in[0,1]$. A function of this form will be maximized at $\zeta=0$ if $a_{2}>a_{1}$, and maximized at $\zeta=1$ if $a_{1}>a_{2}$. Let us show that the maximum acceleration occurs along the line $\theta_{N}=\pi / 2$. That is, let us prove that for the selected $v\left(r_{d}\right)$ of Eq. (5.40), the following inequality holds

$$
\left(\left[1+\frac{\pi}{2 \theta_{d}}\right] \frac{v^{2}\left(r_{d}\right)}{r_{d}}\right)^{2} \geq\left(\frac{d v}{d r_{d}} v\left(r_{d}\right)\right)^{2}
$$

First, using the definition for $v\left(r_{d}\right)$

$$
\left(\left[1+\frac{\pi}{2 \theta_{d}}\right] \frac{v^{2}\left(r_{d}\right)}{r_{d}}\right)^{2} \geq\left(v_{\max } \frac{f \pi}{\alpha^{\prime}} \cos \left(r_{N}\right) v\left(r_{d}\right)\right)^{2}
$$

or, rearranging and collecting terms $f, \pi, \alpha^{\prime}$ and $r_{d}$ into $r_{N}$

$$
\begin{gathered}
\left(\left[1+\frac{\pi}{2 \theta_{d}}\right] v\left(r_{d}\right)\right)^{2} \geq\left(v_{\max } r_{N} \cos \left(r_{N}\right)\right)^{2} \\
\left(\left[1+\frac{\pi}{2 \theta_{d}}\right] \frac{\sin r_{N}}{\cos r_{N}}\right)^{2} \geq r_{N}^{2}
\end{gathered}
$$

Using the Taylor expansion of $\tan r_{N}$ then results in

$$
\left(\left[1+\frac{\pi}{2 \theta_{d}}\right]\left[r_{N}+\ldots\right]\right)^{2} \geq r_{N}^{2}
$$

which is obviously true. Therefore, Eq. (5.51) is maximized when $\theta_{N}=\pi / 2$, and it follows that

$$
\left\|\phi_{1}^{\prime} \hat{\boldsymbol{r}}_{d}+\phi_{2}^{\prime} \hat{\boldsymbol{a}}^{\prime}\right\| \leq\left(1+\frac{\pi}{2 \theta_{d}}\right) \frac{v^{2}\left(r_{d}\right)}{r_{d}}
$$

which, when recharacterized in terms of $r_{N}$

$$
\left\|\phi_{1}^{\prime} \hat{\boldsymbol{r}}_{d}+\phi_{2}^{\prime} \hat{\boldsymbol{a}}^{\prime}\right\| \leq\left(1+\frac{\pi}{2 \theta_{d}}\right)\left(\frac{v_{\max }^{2} f \pi}{\alpha^{\prime}}\right) \frac{\sin ^{2} r_{N}}{r_{N}}
$$


is maximized numerically at $r_{N} \approx 1.16556$, and therefore

$$
\left\|\phi_{1}^{\prime} \hat{\boldsymbol{r}}_{d}+\phi_{2}^{\prime} \hat{\boldsymbol{a}}^{\prime}\right\| \leq 0.724612\left(1+\frac{\pi}{2 \theta_{d}}\right)\left(\frac{v_{\max }^{2} f \pi}{\alpha^{\prime}}\right)
$$

Therefore, using the bound given by Eq. (5.45) and the maximum acceleration of the chaser $u_{\max }$, the acceleration constraint will be respected if the LVF parameters $v_{\max }$ and $\alpha^{\prime}$ are members of the following set

$$
u_{\max } \geq 0.724612\left(1+\frac{\pi}{2 \theta_{d}}\right)\left(\frac{v_{\max }^{2} f \pi}{\alpha^{\prime}}\right)+2 n_{1} v_{\max }+n_{3}+n_{2} \alpha^{\prime}
$$

Convexity of the set $\left(v_{\max }, \alpha^{\prime}\right)$ given by the inequality Eq. (5.61) is easy to show in the following two steps: first, note that the right-hand side of Eq. (5.61) is bounded from above by a constant $u_{\max }$. Then, it is easily shown that the Hessian of the function on the right-hand side of Eq. (5.61) is positive semi-definite when $v_{\max }>0$, $\alpha^{\prime}>0$. Therefore, the set described by Eq. (5.61) is convex.

\subsection{Parameter Optimization Design}

Note that for both Phase 1 and Phase 2 guidance, the set of parameters which guarantee a feasible trajectory form convex sets. Because of this fact, it is possible to build a convex optimizer for parameter selection where Eqs. (5.25) and (5.61) are included as inequality conditions to mandate the acceleration constraint. In this Section, one specific strategy for optimizing these field parameters to minimize some combined cost function of fuel usage and maneuver time is explored. Specifically, this strategy is outlined in the following two steps:

1. the fuel usage for each Phase as measured by the impulse $\Delta \nu$, and the timeduration for each Phase $T$ will be estimated purely as functions of the field parameters $b, k_{c}, k_{a}, \alpha^{\prime}$, and $v_{\max }$.

2. using the (convex) estimates, a constrained optimization of the following form 
will be solved

$$
\begin{aligned}
\min _{\boldsymbol{p}} & W_{f} \Delta \hat{\nu}(\boldsymbol{p})+W_{t} \hat{T}(\boldsymbol{p}) \\
\text { subject to } & p_{i}>0 \\
& u_{\max } \geq u(\boldsymbol{p})
\end{aligned}
$$

where $\boldsymbol{p}$ is the relevant vector of parameters, $W_{i}$ are user-defined weights, $\Delta \hat{\nu}(\boldsymbol{p})$ is an estimate of the fuel usage as a function the field parameters, $\hat{T}(\boldsymbol{p})$ is an estimate of the maneuver time as a function of the field parameters, and the last constraint mandates the acceleration limits (i.e., either Eq. (5.25) or Eq. (5.61) would be used as the final inequality).

Now, it is important to note that there are a few ways in which this optimization approach differs from those seen in MPC or Inverse-Dynamics Trajectory generators (discussed in Sec. 1.3). The first distinguishing fact is the number of optimizations. In both of the approaches listed above, an optimization is performed repeatedly onboard to continuously update the upcoming trajectory. In the case presented here, only two optimizations are performed to select parameters for the first and second trajectory fields. These parameters are then used throughout the entire trajectory.

The next distinguishing fact is the dimension and complexity of the optimization. In MPC, for example, the optimization is performed over a time-horizon of the upcoming trajectory. That is, for each additional time-index $k$ included in the timehorizon, three more variables (the $x, y$ and $z$ position variables for time index $k$ ) are added to the optimization. If real-time control is running at $10 \mathrm{~Hz}$, and the optimization time-horizon is just 1 second into the future, then this will correspond to solving an optimization problem for a 30 by 1 vector every 0.1 seconds. Of course, as the time-horizon increases, or the time-step decreases, this problem is quickly exacerbated. In contrast, the vector of parameters which is optimized using InverseDynamics Trajectory generation are polynomial coefficients, and the optimization is non-convex. In the optimizations presented here, the solutions are a 2 by 1 and a 3 by 1 vector of parameters, and the optimization is convex. Therefore, both the dimension and complexity of this optimization are small compared to existing methods.

The last distinguishing fact are the optimization variables themselves. In MPC, for example, because the optimization variables are thrust inputs, there is direct control 
over the total fuel and time for the maneuver. Unfortunately, in the optimization method presented here, the optimization variables are field parameters which do not directly correspond to fuel usage or maneuver time. All that can be guaranteed by the optimization approach explored in this Section is that the fuel and maneuver time estimates will respond accordingly to the user-defined weights. It must therefore be confirmed that the estimation functions indeed correlate strongly with the true fuel usage and time duration.

The parameter optimization approach will be presented as follows: first, approximation functions for thrust usage and maneuver time based on field parameters are first developed in Sec. 5.4.1. Then, an interior-point optimization algorithm based on those approximations will be presented in Sec. 5.4.3.

\subsubsection{Time and Fuel Approximation Functions}

As the docking approach is divided into two phases, so too will the maneuver time and fuel estimation functions. Specifically, the true alignment time $T_{P 1}$ and fuel usage $\Delta \nu_{P 1}$ will be approximated with functions $\hat{T}_{P 1}\left(b, k_{c}, k_{a}\right)$ and $\Delta \hat{\nu}_{P 1}\left(b, k_{c}, k_{a}\right)$. Likewise, the true contraction time $T_{P 2}$ and fuel usage $\Delta \nu_{P 2}$ will be approximated by the functions $\hat{T}_{P 2}\left(\alpha^{\prime}, v_{\max }\right)$ and $\Delta \hat{\nu}_{P 2}\left(\alpha^{\prime}, v_{\max }\right)$.

\section{Phase 1 Time Approximation}

It will be assumed for this approximation that the maneuver occurs in two distinct steps, namely: the chaser is first brought to the radius $\alpha$ in time $T_{P 11}$, and then afterwards, aligns with the vector $\hat{\boldsymbol{o}}$ in time $T_{P 12}$. This is, of course, not an exact representation of what actually occurs. Nevertheless, this "two-part" Phase 1 will simplify the analysis, and is a reasonable enough approximation of the true guidance.

Assuming a large initial distance, the chaser will spend significant time coasting towards $r=\alpha$ at maximum speed. If $\alpha$ is small compared to the initial separation distance, then the time to contract to the sphere $\alpha$, is estimated by

$$
\hat{T}_{P 11}\left(k_{c}\right)=\frac{\left\|\boldsymbol{r}_{0}\right\|}{k_{c}}
$$


For $T_{P 12}$, it is assumed that the chaser initially lands on the attractor with some misalignment, and must travel an arc length $\zeta$ around the sphere. This is visualized in Fig. 5.4.

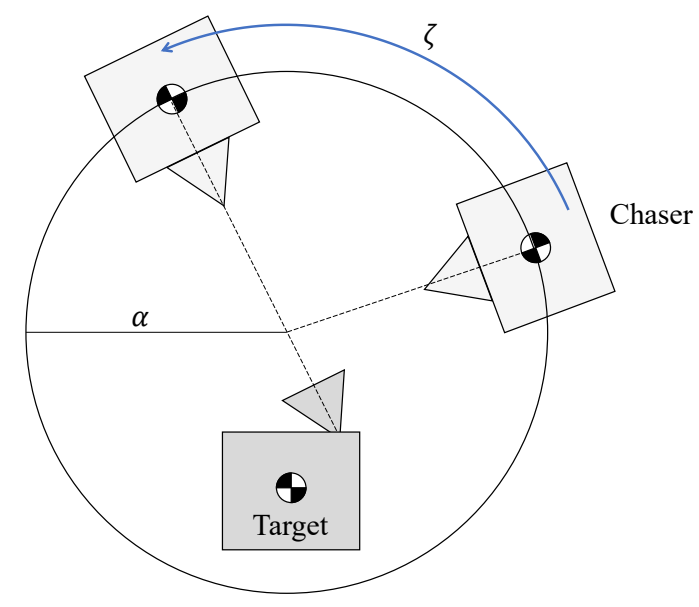

Figure 5.4: Chaser arriving on attractor with initial misalignment.

The chaser will reduce this distance by $d \zeta$ in time $d t$ according to

$$
d \zeta=-s_{a}(\alpha, \theta) d t
$$

or, expressing the distance $d \zeta$ as a change in the alignment angle $\theta$ at the distance $\alpha$ and rearranging

$$
d t=-\alpha \frac{d \theta}{s_{a}(\alpha, \theta)}
$$

Substituting the function $s_{a}$ leads to

$$
d t=-\frac{\alpha}{k_{a}} \frac{d \theta}{\sin \theta}
$$

Assuming that the chaser is initially misaligned by $\pi / 2$ radians on average, the time $T_{P 12}$ can then be estimated

$$
\hat{T}_{P 12}\left(k_{a}\right)=\frac{\alpha}{k_{c}} \int_{0.001 \pi}^{\pi / 2} \frac{d \theta}{\sin \theta}
$$

where $0.001 \pi$ is used because the integral with a limit of 0 is (slowly) divergent, but 
relatively high-accuracy before switching to the contraction phase is assumed. This can then be numerically approximated as

$$
\hat{T}_{P 12}\left(k_{a}\right)=6.5 \frac{\alpha}{k_{a}}
$$

Finally, it is desirable to account for $b$ in the time estimation, as larger distances $b$ corresponds to more time spent below maximum speed. Representing the distance $(r-\alpha)$ by the variable $r_{\alpha}$, a small step can be represented

$$
d r_{\alpha}=-v_{c}\left(r_{\alpha}\right) d t
$$

or, rearranging and substituting the selected function for $v_{c}$, the time to decelerate from the maximum contraction speed $k_{c}$ is given by

$$
\hat{T}_{P 1, \text { decel }}\left(b, k_{c}\right)=\int_{0.001 b}^{b} \frac{d r_{\alpha}^{\prime}}{-\frac{k_{c}}{b^{2}} r_{\alpha}^{2}+2 \frac{k_{c}}{b} r_{\alpha}^{\prime}}
$$

which is unfortunately dependent on $b$ and evaluates to

$$
\hat{T}_{P 1 \text {, decel }}\left(b, k_{c}\right)=\frac{b\left(\log (2 b)-\log \left(\frac{b}{1000}\right)\right)}{2 k_{c}}
$$

Instead, it would be preferred that the expression is convex while still representing that the travel time will increase with $b$ inversely proportional to $k_{c}$. Therefore, a replacement expression is selected as

$$
\hat{T}_{P 1, \text { decel }}\left(b, k_{c}\right)=\frac{b^{2}}{k_{c}}
$$

The final time-estimation function is then given as

$$
\hat{T}_{P 1}\left(b, k_{c}, k_{a}\right)=\frac{\left\|\boldsymbol{r}_{0}\right\|}{k_{c}}+\frac{6.5 \alpha}{k_{a}}+\frac{b^{2}}{k_{c}}
$$

Note that, so long as $b<<\|\boldsymbol{r}\|_{0}$, this adjustment to the time-approximation will not be significant. 


\section{Phase 1 Fuel Approximation}

As is typically the case in spacecraft applications, the fuel usage of a maneuver will be measured according to the required impulse, denoted as a change in velocity $\Delta \nu$. For a given time range $\left(t_{1}, t_{2}\right)$, this is measured as

$$
\Delta \nu=\int_{t_{1}}^{t_{2}}\|\ddot{\boldsymbol{r}}\| d t
$$

As with the time estimation, the fuel consumption will be approximated as the sum of two sub-maneuvers which are performed in sequence to achieve Phase 1. Specifically, $\Delta \nu_{P 11}$ represents the fuel cost to correct the initial velocity error to the desired coasting velocity, and then decelerate back to zero velocity as $r \rightarrow \alpha$. Likewise, $\Delta \nu_{P 12}$ will represent the fuel required to align with $\hat{\boldsymbol{o}}$ upon arrival to the radius $\alpha$.

The fuel usage $\Delta \nu_{P 11}$ can therefore be calculated by summing the initial velocity error with the total deceleration required to bring the contraction speed to 0. This is visualized in Fig. 5.5, and the estimated fuel usage for these maneuvers is given as

$$
\Delta \hat{\nu}_{P 11}\left(k_{c}\right)=\left\|\dot{\boldsymbol{r}}_{0}+k_{c} \hat{\boldsymbol{r}}_{0}\right\|+k_{c}
$$

To estimate the fuel required to align with $\hat{\boldsymbol{o}}$ after arrival to the radius $\alpha$, begin by substituting Eq. (5.65) for $d t$ into Eq. (5.74), resulting in

$$
\Delta \nu_{P 12}=\alpha \int_{\theta_{1}}^{\theta_{2}} \frac{\|\ddot{\boldsymbol{r}}(\theta)\|}{s_{a}(\alpha, \theta)} d \theta
$$

or, expanding $s_{a}(\alpha, \theta)$

$$
\Delta \nu_{P 12}=\frac{\alpha}{k_{c}} \int_{\theta_{1}}^{\theta_{2}} \frac{\|\ddot{\boldsymbol{r}}(\theta)\|}{\sin \theta} d \theta
$$

Therefore, what remains is to express the total required acceleration as a function of the alignment angle, and then to perform this integral. To attain $\ddot{\boldsymbol{r}}(\theta)$, begin with the expression

$$
\ddot{\boldsymbol{r}}=\boldsymbol{\omega}_{B I}^{\times} \boldsymbol{\omega}_{B I}^{\times} \boldsymbol{r}+\dot{\boldsymbol{\omega}}_{B I}^{\times} \boldsymbol{r}+2 \boldsymbol{\omega}_{B I}^{\times}\left(v_{c}(\alpha, \theta) \hat{\boldsymbol{r}}+s_{a}(\alpha, \theta) \hat{\boldsymbol{a}}\right)+\phi_{1}(\alpha, \theta) \hat{\boldsymbol{r}}+\phi_{2}(\alpha, \theta) \hat{\boldsymbol{a}}
$$




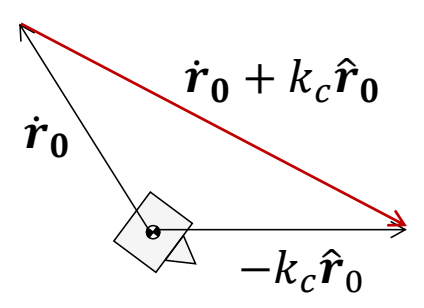

Initial
velocity
correction

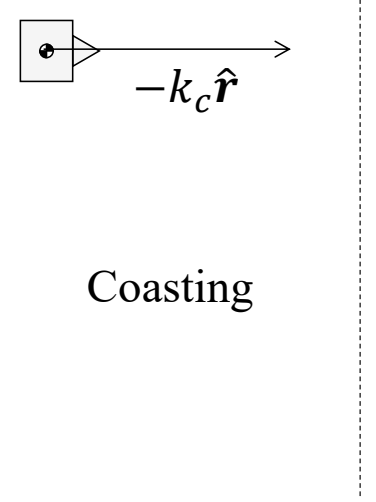

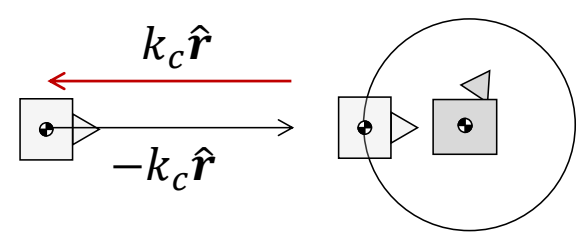

Deceleration to attractor

Figure 5.5: Two $\Delta \nu$ maneuvers to contract to the radius $\alpha$.

where $\phi_{1}(\alpha, \theta)$ and $\phi_{2}(\alpha, \theta)$ evaluate to the familiar form of relative normal and tangential accelerations when $r=\alpha$. Specifically

$$
\phi_{1}(\alpha, \theta) \hat{\boldsymbol{r}}+\phi_{2}(\alpha, \theta) \hat{\boldsymbol{a}}=-\frac{k_{a}^{2} \sin ^{2} \theta}{\alpha} \hat{\boldsymbol{r}}+\frac{k_{a}^{2} \sin \theta \cos \theta}{\alpha} \hat{\boldsymbol{a}}
$$

which has the norm

$$
\left\|\phi_{1}(\alpha, \theta) \hat{\boldsymbol{r}}+\phi_{2}(\alpha, \theta) \hat{\boldsymbol{a}}\right\|=\frac{k_{a}^{2} \sin \theta}{\alpha}
$$

Using the observed metrics of the target rotation, namely, $n_{1}$ and $n_{2}$, the acceleration function is conservatively estimated by

$$
\|\ddot{\boldsymbol{r}}(\theta)\| \approx n_{2} \alpha+2 n_{1} k_{a} \sin \theta+\frac{k_{a}^{2} \sin \theta}{\alpha}
$$

Note that, although this fuel estimate may be unrealistic, the goal of this optimization is not to produce exact fuel estimates. Rather, the fuel estimate function only needs to have a strong correlation with true fuel usage. Substituting Eq. (5.81) into Eq. 
(5.77) results in

$$
\Delta \hat{\nu}_{P 12}\left(k_{a}\right)=\frac{\alpha}{k_{a}} \int_{\theta_{1}}^{\theta_{2}}\left(\frac{n_{2} \alpha}{\sin \theta}+2 n_{1} k_{a}+\frac{k_{a}^{2}}{\alpha}\right) d \theta
$$

which, again assuming that the chaser must on average sweep through one quarter of the spherical attractor leads to the selection of $\theta_{1}=\pi / 2$ and $\theta_{2}=0.001 \pi$, evaluating numerically to

$$
\Delta \hat{\nu}_{P 12}\left(k_{a}\right)=\left(2 n_{1} \alpha+k_{a}\right) \frac{\pi}{2}+\frac{6.5 \alpha^{2} n_{2}}{k_{a}}
$$

leading to the final fuel estimate for the alignment phase

$$
\Delta \hat{\nu}_{P 1}\left(k_{c}, k_{a}\right)=\left\|\dot{\boldsymbol{r}}_{0}+k_{c} \hat{\boldsymbol{r}}_{0}\right\|+k_{c}+\left(2 n_{1} \alpha+k_{a}\right) \frac{\pi}{2}+\frac{6.5 \alpha^{2} n_{2}}{k_{a}}
$$

\section{Phase 1 Jerk Penalty}

It is undesirable to produce guidance commands which have an overly high derivative in their acceleration commands. Therefore, a third term is added to the cost function to prevent $b$ from becoming too small, especially for large $k_{c}$. This is referred to as the jerk penalty $\chi$, and is given by

$$
\chi=\frac{k_{c}^{2}}{b}
$$

\section{Phase 2 Time Estimation}

Similar to the previous approximations, some assumptions regarding the spacecraft behaviour will be employed. Primarily, it is assumed that upon completion of Phase 1, the chaser begins Phase 2 in very good alignment with the target docking port vector $\hat{\boldsymbol{o}}^{\prime}$. Following the expected behaviour, it is then assumed that the chaser remains in good alignment. Therefore, the maneuver time is estimated assuming a trajectory which is a pure contraction along the $\hat{o}^{\prime}$ line. A small reduction step $d r_{d}$ along this line is taken by

$$
d r_{d}=-v\left(r_{d}, 0\right) d t
$$


Or, substituting for the normalized distance $r_{N}$ and rearranging for $d t$

$$
d t=-\frac{\alpha^{\prime}}{f \pi} \frac{d r_{N}}{v\left(r_{N}, 0\right)}
$$

substituting the function for $v\left(r_{N}, 0\right)$ and integrating then results in

$$
\hat{T}_{P 2}\left(\alpha^{\prime}, v_{\max }\right)=\frac{\alpha^{\prime}}{f \pi v_{\max }} \int_{0.01 \pi}^{f \pi} \frac{d r_{N}}{\sin r_{N}}
$$

where $0.01 \pi$ is used as the normalized distance measurement which indicates successful docking. Assuming a conservative value of $f=0.99$ is used for the integration limit, the time estimate results in

$$
\hat{T}_{P 2}\left(\alpha^{\prime}, v_{\max }\right)=8.31 \frac{\alpha^{\prime}}{f \pi v_{\max }}
$$

\section{Phase 2 Fuel Estimate}

To begin, Eq. (5.87) is substituted for $d t$ in the definition of $\Delta \nu$ to obtain

$$
\Delta \nu_{P 2}=\frac{\alpha^{\prime}}{f \pi} \int_{r_{N, 1}}^{r_{N, 2}} \frac{\left\|\ddot{\boldsymbol{r}}\left(r_{N}\right)\right\|}{v\left(r_{N}\right)} d r_{N}
$$

therefore, it is necessary to estimate the required acceleration as a function of the normalized distance $r_{N}$. Beginning with the relative acceleration equation for a rotating reference frame

$$
\begin{array}{r}
\ddot{\boldsymbol{r}}_{d}=\boldsymbol{\omega}_{B I}^{\times} \boldsymbol{\omega}_{B I}^{\times}\left(\boldsymbol{d}+\boldsymbol{r}_{d}\right)+\dot{\boldsymbol{\omega}}_{B I}^{\times}\left(\boldsymbol{d}+\boldsymbol{r}_{d}\right)+2 \boldsymbol{\omega}_{B I}^{\times}\left(v_{c}\left(r_{d}, 0\right) \hat{\boldsymbol{r}}+s_{a}\left(r_{d}, 0\right) \hat{\boldsymbol{a}}\right) \\
+\phi_{1}^{\prime}\left(r_{d}, 0\right)^{\prime} \hat{\boldsymbol{r}}_{d}+\phi_{2}^{\prime}\left(r_{d}, 0\right) \hat{\boldsymbol{a}}^{\prime}
\end{array}
$$

Using the observed maximum attitude dynamics characteristics, and the fact that $r_{N}=\frac{r_{d}}{\alpha^{\prime}} f \pi$ inside the radius $r_{d}<\alpha^{\prime}$ the acceleration norm for tracking the Phase 2 guidance inside of the tumbling reference frame is conservatively estimated by

$$
\left\|\ddot{\boldsymbol{r}}_{d}\left(r_{N}\right)\right\|=n_{3}+n_{2} \frac{\alpha^{\prime}}{f \pi} r_{N}+2 n_{1} v_{\max } \sin r_{N}+\left\|\phi_{1}^{\prime}\left(r_{d}, 0\right)^{\prime} \hat{\boldsymbol{r}}_{d}+\phi_{2}^{\prime}\left(r_{d}, 0\right) \hat{\boldsymbol{a}}^{\prime}\right\|
$$


where the same note applies that, although the fuel estimate is conservative, so long as it correlates to true fuel usage, this estimate will be useful for an optimization. Evaluating $\left\|\phi_{1}^{\prime}\left(r_{d}, 0\right)^{\prime} \hat{\boldsymbol{r}}_{d}+\phi_{2}^{\prime}\left(r_{d}, 0\right) \hat{\boldsymbol{a}}^{\prime}\right\|$ results in

$$
\left\|\phi_{1}^{\prime}\left(r_{d}, 0\right)^{\prime} \hat{\boldsymbol{r}}_{d}+\phi_{2}^{\prime}\left(r_{d}, 0\right) \hat{\boldsymbol{a}}^{\prime}\right\|=\frac{v_{\max }^{2} f \pi}{\alpha^{\prime}} \sin r_{N} \cos r_{N}
$$

which, when substituted back in Eq. (5.90) results in

$\Delta \hat{\nu}_{P 2}\left(\alpha^{\prime}, v_{\max }\right)=\frac{\alpha^{\prime}}{f \pi v_{\max }} \int_{r_{N, 1}}^{r_{N, 2}}\left(\frac{n_{3}}{\sin r_{N}}+\frac{n_{2} \alpha^{\prime} r_{N}}{f \pi \sin r_{N}}+2 n_{1} v_{\max }+\frac{v_{\max }^{2} f \pi}{\alpha^{\prime}} \cos r_{N}\right) d r_{N}$

using bounds of $r_{N, 1}=0.01 \pi$ and $r_{N, 2}=0.99 \pi$ for the divergent integrals results in

$$
\Delta \hat{\nu}_{P 2}\left(\alpha^{\prime}, v_{\max }\right)=\frac{\alpha^{\prime}}{f \pi}\left(8.31 \frac{n_{3}}{v_{\max }}+13.05 \frac{n_{2} \alpha^{\prime}}{f \pi v_{\max }}+2 \pi n_{1}+\frac{2 v_{\max } f \pi}{\alpha^{\prime}}\right)
$$

\subsubsection{Optimization Cost Function}

In this Section, the cost functions to be minimized by the optimizer are outlined. In Sec. 5.4.3, an interior point optimizer using a Newton-step will be developed to minimize this cost function. However, note that in a number of other optimization techniques could be used, which is why the cost functions are presented independently.

For each of Phase 1 and Phase 2, the cost will be divided into two segments. Specifically, the cost is partially composed of a (convex) function of performance $J$, and partially composed of a penalty term, $P$ which increases to infinity as constraints are approached. That is

$$
C=\lambda J+P
$$

where $\lambda \in \mathbb{R}>0$ is a factor which weighs the relative importance of the performance and constraint costs. One broadly utilized penalty function $P$ is the so-called logbarrier function, given by

$$
P=-\sum_{i=1}^{M} \log s_{i}
$$

where $s_{i}>0$ are the components of a "slack vector" $s \in \mathbb{R}^{M}$. Values of $s_{i}$ which approach 0 correspond to constraints which are approaching violation. As is clear 
from Eq. (5.97), constraints which approach violation will tend towards an infinite penalty. It should be noted that, when used precisely, arguments of logarithmic or exponential functions should be dimensionless. However, as is the case in most engineering works that utilize slack vectors, this mathematical detail is not considered here. The effect of ignoring this detail is that the cost penalty becomes dependent on the selected units of the slack-variable. The optimal set of parameters, however, will not change significantly so long as $\lambda$ is large.

\section{Phase 1 Cost Function}

In Phase 1, the parameter vector $\boldsymbol{p}_{P 1}=\left[\begin{array}{lll}b & k_{c} & k_{a}\end{array}\right]^{T}$ will be optimized. The distance $\alpha$ is not included in this optimization as it is geometrically determined from $\alpha^{\prime}$. The performance cost function $J$ is then selected as

$$
J_{P 1}=W_{t, P 1} \hat{T}_{P 1}\left(b, k_{c}, k_{a}\right)+W_{f, P 1} \Delta \hat{\nu}_{P 1}\left(k_{c}, k_{a}\right)+W_{\chi, P 1} \chi\left(k_{c}, b\right)
$$

where $W_{i}$ are various strictly positive adjustable weights. The slack vector to be included within the log-barrier function $P$ is as follows

$$
\boldsymbol{s}_{P 1}=\left[\begin{array}{c}
b \\
k_{c} \\
k_{a} \\
u_{\max }-u_{\mathrm{P} 1}\left(b, k_{c}, k_{a}\right)
\end{array}\right]
$$

where

$$
u_{\mathrm{P} 1}=\sqrt{\left(\frac{k_{c}^{2}}{b}+\frac{k_{a}^{2}}{\alpha}\right)^{2}+\left(\frac{k_{a}^{2}}{2 \alpha}+\frac{2}{3 \sqrt{3}} k_{a} n_{1}\right)^{2}}+2.385 k_{a} n_{1}+n_{2} \alpha
$$

The first three indices of $\boldsymbol{s}_{P 1}$ enforce positive values for $b, k_{c}$ and $k_{a}$, and the last index enforces parameter selection which obeys the acceleration constraints. Note that the penalty function is convex in $b, k_{c}$ and $k_{a}$. This can be shown by the fact that

$$
P_{P 1}=-\left(\log b+\log k_{c}+\log k_{a}+\log \left(u_{\max }-u_{P 1}\left(b, k_{c}, k_{a}\right)\right)\right)
$$


is composed entirely of terms of the form

$$
P_{i}=-\log \left(\zeta\left(b, k_{c}, k_{a}\right)\right)
$$

Noting that the function $P_{i}=-\log \zeta$ is convex and strictly decreasing in $\zeta$, then $P_{i}$ is convex in $\left(b, k_{c}, k_{a}\right)$ if $\zeta$ is concave in $\left(b, k_{c}, k_{a}\right)$ by convex function composition rules [34]. The final cost function for Phase 1 is then given by

$$
C_{P 1}=\lambda J_{P 1}-\frac{1}{4} \sum_{i=1}^{4} \log s_{P 1, i}
$$

where the factor of $1 / 4$ results in some measure of the "average closeness" to constraint violation.

\section{Phase 2 Cost Function}

To begin, it is noted that the Phase 2 fuel estimate Eq. (5.95) is convex in $v_{\max }$ and $\alpha^{\prime}$ individually, but not jointly.

In most cases, unless an unusually high value for $v_{\max }$ is selected, the optimal $\alpha^{\prime}$ for Phase 2 time and fuel minimization alike will be the smallest value which is considered operationally safe. Furthermore, as noted in Eqs. (5.73) and (5.84), both the time and fuel of Phase 1 strictly increase with increasing $\alpha$, which is geometrically determined by $\alpha^{\prime}$. As will be seen in the simulation and experimental data, Phase 1 tends to cost significantly more time and fuel than Phase 2, and therefore selecting smaller values of $\alpha^{\prime}$ (and by extension, $\alpha$ ) is beneficial to the overall maneuver performance. Therefore, to maintain a convex optimization for Phase 2, and reduce the number of optimization variables, it is assumed that $\alpha^{\prime}$ is set to the minimum safe value, and only $v_{\max }$ is optimized. The performance cost for Phase 2 is therefore given purely as a function of $v_{\max }$

$$
J_{P 2}=W_{t, P 2} \hat{T}_{P 2}\left(v_{\max }\right)+W_{f, P 2} \Delta \hat{\nu}_{P 1}\left(v_{\max }\right)
$$

where $W_{i}$ are various strictly positive adjustable weights. The slack vector for Phase 
2 to be included within the log-barrier function $P_{P 2}$ is

$$
\boldsymbol{s}_{P 2}=\left[\begin{array}{c}
v_{\max } \\
u_{\max }-u_{\mathrm{P} 2}\left(v_{\max }\right)
\end{array}\right]
$$

where

$$
u_{\mathrm{P} 2}=0.7246\left(1+\frac{\pi}{2 \theta_{d}}\right)\left(\frac{v_{\max }^{2} f \pi}{\alpha^{\prime}}\right)+2 n_{1} v_{\max }+n_{3}+n_{2} \alpha^{\prime}
$$

The first index of $\boldsymbol{s}_{P 2}$ enforces a positive selection for $v_{\max }$, and the second index enforces the acceleration constraint. The final cost function for Phase 2 is then given by

$$
C_{P 2}=\lambda J_{P 2}-\frac{1}{2} \sum_{i=1}^{2} \log s_{P 2, i}
$$

\subsubsection{Interior Point Optimizer with Newton Step Design}

This Section outlines an interior point optimizer for selection of the parameters $v_{\max }$, $k_{a}, k_{c}$ and $b$. It should be noted that an interior point optimization does not rely on using a Newton-step; for example, a gradient descent could also be used within the framework of interior point optimization. For this reason, the interior point algorithm and the Newton-step algorithm are reviewed separately. As the specific optimization technique used to minimize the cost functions Eq. (5.103) and Eq. (5.107) is not of significance to the theory developed in this thesis, only a high-level review of interior point optimization and the Newton-step will be given. For a very detailed explanation, see $[35]$.

In the presentation of this Section, a generic cost function $C$ and parameter vector $\boldsymbol{p}$ are considered, as the arguments apply generally to both Phase 1 and Phase 2 . Likewise, the performance cost and constraint penalty functions are generic, and referred to simply as $J$ and $P$. Note that the gradient and Hessian of the Phase 1 and Phase 2 cost functions will be required; these are readily attainable through softwares such as MATLAB symbolic or Maple, and as they do not provide any theoretical insight, the actual expressions for the cost gradient and Hessians are given in Appendix C. 


\section{Interior Point Algorithm}

Given a parameter $\beta \in \mathbb{R}>1$ and a large number $\Lambda \in \mathbb{R}$, the interior point optimization is given by [35].

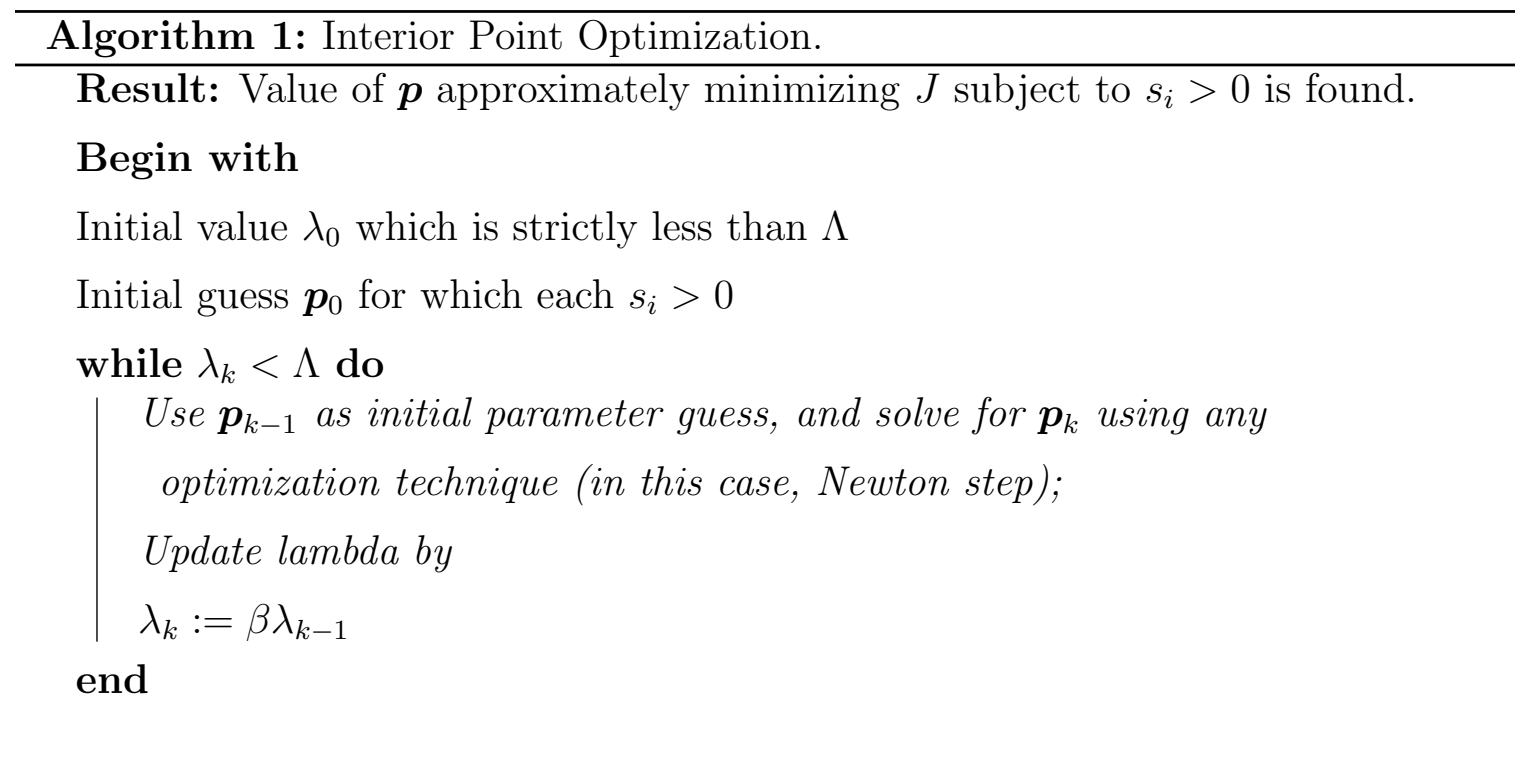

Intuitively, by increasing the value of $\lambda$ to a high value $\Lambda$, the vector $\boldsymbol{p}$ which minimizes $C$ will closely approximate a minimizer for $J$ subject to the conditions that $s_{i}>0$.

\section{Newton-step}

The Newton-step is selected as the optimizer due to its fast performance with convex functions, and in particular, its local quadratic convergence [35]. It should be noted that for problems of high dimension, the Newton-step can become too costly to implement on-board spacecraft in real-time due to the need to compute a Hessian and gradient, and solve a system of linear equations. However, given that the present optimization solves for a parameter vector of at most dimension $\mathbb{R}^{3}$, and that the optimization need only be solved once, the computational burden of this optimization is very low.

Considering an optimization index $k$ which counts the total number of optimization steps which have been taken while solving for a minimum, the Newton-step 
algorithm updates $\boldsymbol{p}_{k}$ as

$$
\boldsymbol{p}_{k}=\boldsymbol{p}_{k-1}-\gamma \frac{\partial^{2} C}{\partial \boldsymbol{p}^{2}} \frac{\partial C^{T}}{\partial \boldsymbol{p}}
$$

where $0<\gamma \leq 1$ is often referred to as a "dampening factor", and $\frac{\partial^{2} C}{\partial \boldsymbol{p}^{2}}$ and $\frac{\partial C^{T}}{\partial \boldsymbol{p}}$ are the Hessian and gradient of $C$ respectively evaluated at the estimate $\boldsymbol{p}_{k-1}$. The estimate $\boldsymbol{p}_{k}$ is then updated according to Eq. (5.108) until some sufficiently small gradient magnitude $\left\|\frac{\partial C^{T}}{\partial \boldsymbol{p}}\right\|$ is achieved.

\subsection{Switching Conditions}

This work does not focus primarily on the switching conditions. Therefore, for the sake of simplicity, a method based on the length of time spent within a small radius of the point $\boldsymbol{r}=\alpha \hat{\boldsymbol{o}}$ is used. Specifically, after a time $\tau>0$ is spent within a radius $\rho>0$ of the point $\boldsymbol{r}=\alpha \hat{\boldsymbol{o}}$, the control mode switches. The switching algorithm is therefore

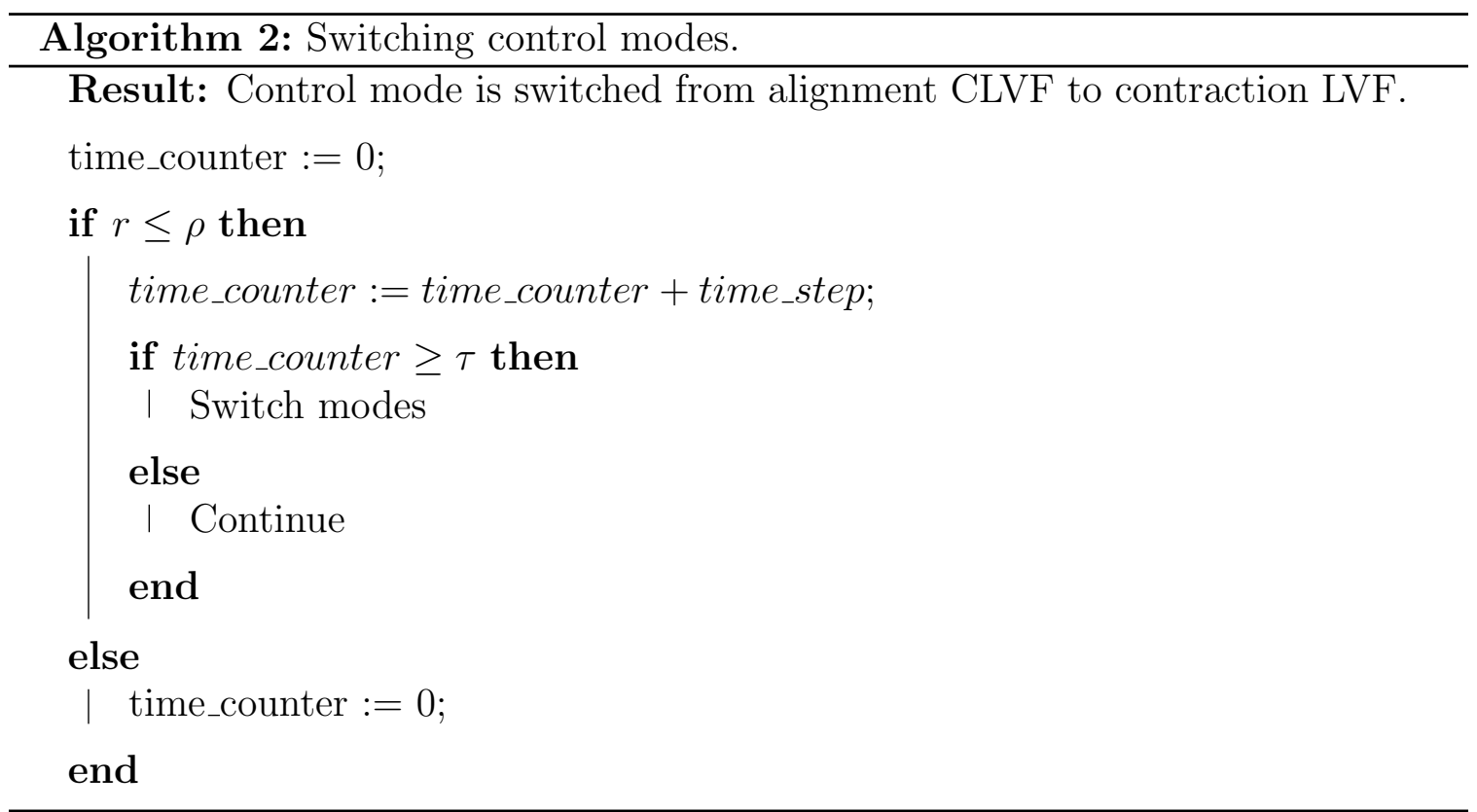




\section{Chapter 6}

\section{Simulations}

In this Chapter, the design of the docking maneuver developed in Chapter 5 is tested under various initial conditions, acceleration constraints, and cost function weights.

\subsection{Simulation Scenario}

Across each of the simulations considered, the target will have constant physical parameters (such as location and size of the docking port, moment of inertia matrix) and constant orbital parameters. Specifically, the target physical parameters and orbital elements are listed in Tables 6.1 and 6.2 respectively. Note that, beyond the acceleration requirements of the maneuver, the chaser will also compensate for the differences in gravity and $J_{2}$ perturbations experienced between both spacecraft. As will be seen, these additional accelerations are quite insignificant compared to the accelerations experienced from the maneuver when the two spacecraft are initiated close relative to their orbital distances, and the maneuver takes place over timescale which are shorter than one orbit.

Table 6.1: Target dynamic and geometric parameters.

\begin{tabular}{cccccc}
\hline \hline $\boldsymbol{J}^{B}\left(\mathrm{~kg} \cdot \mathrm{m}^{2}\right)$ & $\hat{\boldsymbol{o}}^{\prime}$ & $\boldsymbol{d}^{B}(\mathrm{~m})$ & $\theta_{d}(\operatorname{deg})$ & $\alpha^{\prime}(\mathrm{m})$ \\
\hline $\operatorname{diag}(35,40,55)$ & {$\left[\begin{array}{lll}1 & 0 & 0\end{array}\right]^{T}$} & {$\left[\begin{array}{lll}6 & 3 & 0\end{array}\right]^{T}$} & 30 & 4.8 \\
\hline \hline
\end{tabular}

Table 6.2: Target orbit parameters.

\begin{tabular}{cccccc}
\hline \hline$œ_{a}(\mathrm{~km})$ & $œ_{e}$ & $\varlimsup_{i}(\mathrm{deg})$ & $\varlimsup_{\Omega}(\mathrm{deg})$ & $\varlimsup_{\omega}(\mathrm{deg})$ & $\varlimsup_{\theta}(\mathrm{deg})$ \\
\hline 7500 & 0.01 & 0 & 0 & 0 & 0 \\
\hline \hline
\end{tabular}

From $\hat{\boldsymbol{o}}^{\prime}$ and $\alpha^{\prime}$, it is geometrically determined that $\alpha=11.2089 \mathrm{~m}$ and $\hat{\boldsymbol{o}}^{B}=$ 
$\left[\begin{array}{lll}0.9635 & 0.2676 & 0\end{array}\right]^{T}$. The target spacecraft begins in an orientation where the bodyfixed frame is parallel to the inertial frame in every circumstance, and only the initial angular velocity vector is varied. Seven different initial condition sets are tested, listed in Table 6.3. Given that an uncontrolled target will inevitably converge to a major axis spin due to energy losses, the test cases are all perturbed major axis spins of increasing magnitude.

Table 6.3: Simulation initial conditions.

\begin{tabular}{|c|c|c|c|c|c|c|}
\hline Sim Case & $\bar{\omega}_{\boldsymbol{\omega}}^{\boldsymbol{\omega}_{B I, 0}^{B}(\operatorname{degs} / \mathrm{s})}$ & \multicolumn{3}{|c|}{$\boldsymbol{r}_{0}(\mathrm{~m})$} & \multicolumn{2}{|c|}{$\dot{\boldsymbol{r}}_{0}(\mathrm{~m} / \mathrm{s})$} \\
\hline 1 & {$\left[\begin{array}{lll}0.2 & 0.05 & -0.45\end{array}\right]^{T}$} & {$[300$} & -300 & $500]^{T}$ & {$\left[\begin{array}{ll}0.0 & 0.0\end{array}\right.$} & $0.0]^{T}$ \\
\hline 2 & {$\left[\begin{array}{lll}-1.4 & 1.0 & 2.0\end{array}\right]^{T}$} & {$[900$} & -1500 & $-900]^{T}$ & {$[0.0$} & $0.0]^{T}$ \\
\hline 3 & {$\left[\begin{array}{lll}-0.2 & 0.8 & 3.1\end{array}\right]^{T}$} & {$[30$} & -50 & $-30]^{T}$ & {$\left[\begin{array}{ll}0.0 & 0.0\end{array}\right.$} & $0.0]^{T}$ \\
\hline 4 & {$\left[\begin{array}{lll}-1.4 & 1.0 & 4.0\end{array}\right]^{T}$} & {$[300$} & -500 & $-300]^{T}$ & {$\left[\begin{array}{ll}30 & -50\end{array}\right.$} & $10]^{T}$ \\
\hline 5 & {$\left[\begin{array}{lll}1.0 & -1.4 & 5.0\end{array}\right]^{T}$} & {$[50$} & -30 & $-30]^{T}$ & {$\left[\begin{array}{ll}10 & -5.7\end{array}\right.$} & $3.1]^{T}$ \\
\hline 6 & {$\left[\begin{array}{lll}1.0 & -3.4 & 7.0\end{array}\right]^{T}$} & {$[5000$} & -3000 & $-3000]^{T}$ & {$\left[\begin{array}{ll}-2.0 & 3.0\end{array}\right.$} & $1.0]^{T}$ \\
\hline 7 & {$\left[\begin{array}{lll}1.0 & -2.4 & 10.0\end{array}\right]^{T}$} & {$[3000$} & -3000 & $5000]^{T}$ & {$\left[\begin{array}{ll}0.0 & 0.0\end{array}\right.$} & $0.0]^{T}$ \\
\hline
\end{tabular}

For each initial condition, there are five different maximum acceleration constraints which are tested. Specifically, the chaser is given acceleration constraints evenly spaced between $u_{\max }=1 \mathrm{~m} / \mathrm{s}^{2}$ and $u_{\max }=5 \mathrm{~m} / \mathrm{s}^{2}$. Lastly, for each initial condition and acceleration constraint, five different sets of weights are tested. These weights vary between those which very heavily penalize maneuver time to those which heavily penalize fuel usage, and are listed in Table 6.4. Note that the "jerk" is not heavily penalized, but is nonetheless included such that $b$ does not become too small for large values of $k_{c}$. 
Table 6.4: Simulation weights.

\begin{tabular}{cccccccc}
\hline \hline & \multicolumn{3}{c}{ Phase 1} & & \multicolumn{2}{c}{ Phase 2} \\
\cline { 1 - 4 } \cline { 6 - 7 } Weight Case & $W_{t, P 1}$ & $W_{f, P 1}$ & $W_{\chi, P 1}$ & & $W_{t, P 2}$ & $W_{f, P 2}$ \\
\hline 1 & 1.0 & 0.1 & $1 \mathrm{e}-7$ & 1 & 0.001 \\
2 & 1.0 & 1.0 & $1 \mathrm{e}-6$ & 1 & 0.1 \\
3 & 0.1 & 1.0 & $1 \mathrm{e}-7$ & 1 & 1 \\
4 & 0.01 & 1.0 & $1 \mathrm{e}-7$ & 0.01 & 1.0 \\
5 & 0.001 & 1.0 & $1 \mathrm{e}-8$ & 0.001 & 1.0 \\
\hline \hline
\end{tabular}

For the interior point optimizer, $\Lambda$ is set to 1000 . For the velocity controller, the constant gain $\beta$ is set to 0.5 . Setting $\beta$ to this value ensured that the velocity error dynamics were much faster than the time to converge to the docking port in Phase 2 , preventing tracking errors during the final contact. The maneuver switches from Phase 1 to Phase 2 when the conditions $|r-\alpha| \leq 0.05 \mathrm{~m}$ and $\theta \leq 0.5^{\circ}$ are maintained for 5 seconds.

\subsection{Results}

The results are presented in two segments. Specifically, Sec. 6.2.1 shows results pertaining to the acceleration constraints and path constraints for several cases. Then, in Sec. 6.2.2, the variation in performance under the various weights are presented. The correlation between the estimated fuel and maneuver times with the actual values is also studied.

\subsubsection{Acceleration Constraint and Path Constraint Results}

As expected, the acceleration constraint is obeyed in all cases, except for where saturation occurs during initial velocity error correction as discussed in Sec. 3.3.4. Note that these acceleration commands include compensation for differences in gravity and perturbations from $J_{2}$. Likewise, the docking path constraints are all met in each case. Namely, the chaser converges to the distance $\alpha=11.2098 \mathrm{~m}$ from the chaser before aligning the with docking port $(\theta \rightarrow 0)$. Then, the chaser switches to Phase 2, where 
it converges into the docking position while keeping $\theta^{\prime} \leq \theta_{d}$.

As it would be unreasonable to show the acceleration and path constraint results for every case, only 15 specific cases are shown (the full 175 cases are all utilized in Sec. 6.2.2). Specifically, the most time-restricted cases (i.e. the first weight case) are shown, for initial condition cases 1, 4 and 7 in Figs. 6.1-6.15. To assist in visualization of the trajectory, a time-lapse of initial condition set 4 using weight set 1 with $u_{\max }=$ $1.0 \mathrm{~m} / \mathrm{s}^{2}$ is provided in Fig. 6.16.

By comparing results for the identical initial conditions while $u_{\max }$ is varied, such as Figs. 6.1-6.5, or Figs. 6.6-6.10, or Figs. 6.11-6.15, it is clear that the optimization always correctly selects parameters which respect the acceleration constraints. Moreover, the alignment phase results show significant variation in $\theta$ until $r$ has converged to the distance $\alpha$, as expected. It can be seen from the acceleration commands that during this phase of significant $\theta$ variation, nearly no fuel is spent, as the chaser spacecraft passively drifts in towards the target while ignoring its attitude dynamics.

Likewise, Phase 2 (the LVF) shows convergence of $r_{d}$ to 0 while $\theta^{\prime}$ remains well below the limit $\theta_{d}$. It should be noted that the calculated angle $\theta^{\prime}$ "bounces" slightly around 0 . This is simply because a small number has been added into the denominator to avoid divisions by exactly 0 when computing $\theta^{\prime}$. This small number makes no noticeable change to the performance results, but adds significant robustness to division by 0 , and so it is included and this small oscillation in the value of $\theta^{\prime}$ near 0 is accepted.

Another observation is that fuller usage of the chaser acceleration capabilities tends to occur in Phase 1 rather than in Phase 2. As this occurs consistently over the 175 tested simulation cases, it is safe to assume that the acceleration bound for the CLVF given by Eq. (5.25) is tighter than the LVF bound given by Eq. (5.61). This can be explained through the method by which these bounds were obtained. Specifically, these bounds were both solved by maximizing individual groups of terms, and then adding those bounds together to form an absolute bound. In the case of the CLVF, this is a reasonable assumption. That is, most acceleration terms tend to increase as $r \rightarrow \alpha$, and so adding the bounds of the terms individually leads to a reasonably tight bound overall. In the case of the LVF, however, the different acceleration terms 
hit their maximums at different points in the field. For example, the Coriolis term will reach its maximum at the half-way point to the docking cone, where $v\left(r_{N}\right)$ hits a maximum. However, the centripetal and tangential acceleration terms will hit their maximums at $r_{d}=\alpha^{\prime}$, where the distance is greatest. Furthermore, the relative acceleration term of Eq. (5.60) occurs at the maximum cone angle $\theta^{\prime}=\theta_{d}$, which under nominal circumstances is never even reached. It is certainly important that the chaser be able to handle all of these acceleration constraints. For example, should the chaser somehow be perturbed out of alignment during Phase 2, it is necessary that it has sufficient thrust to realign itself for robustness purposes. Nevertheless, it would be an improvement to the LVF portion if a tighter acceleration bound were to be attained.

Overall, the simulation results confirm that the new method is capable of completing docking while meeting all path and acceleration constraints. Importantly, these constraints are not enforced through computationally heavy in-the-loop optimization. Rather, the constraints are only enforced through judicious design of the CLVF for Phase 1 and the LVF for Phase 2.

\subsubsection{Parameter Optimization Results}

To compare the effects of weight variation in the optimizer on the time and fuel metrics, the values of $\Delta \nu_{P 1}, T_{P 1} \Delta \nu_{P 2}$, and $T_{P 2}$ were tabulated for each of the 175 simulation cases. Specifically, the Phase 1 fuel usage and time are tabulated for each weight case in Tables 6.5-6.9 and Tables 6.10-6.14 respectively. Similarly, Tables 6.156.19 and 6.20-6.24 tabulate the Phase 2 fuel usage and maneuver time respectively for each weight case. The tables are shown all together at the end of this Section for convenience.

Overall, the parameter optimization algorithm developed performs very well for cases with lower angular velocities, and still quite well for higher angular velocities so long as the disparity between $W_{f}$ and $W_{t}$ is not too significant. The exact limit where the weight disparity has adverse effects on performance is not yet known, but some observations and potential causes for this effect will be discussed for Phases 1 and 2 independently further on in this Section. 


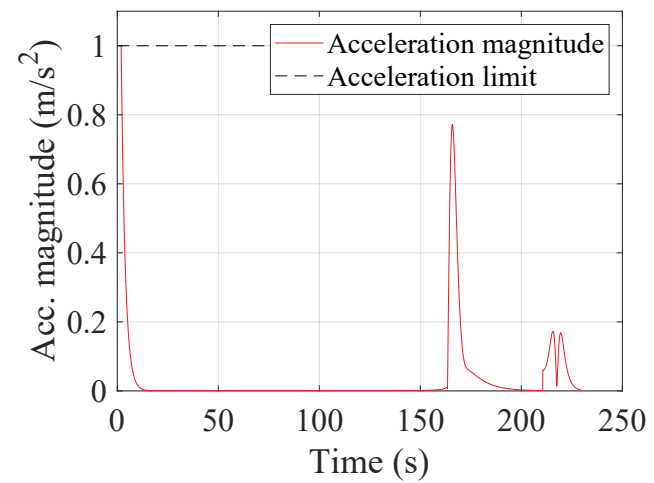

(a) Acceleration commands.

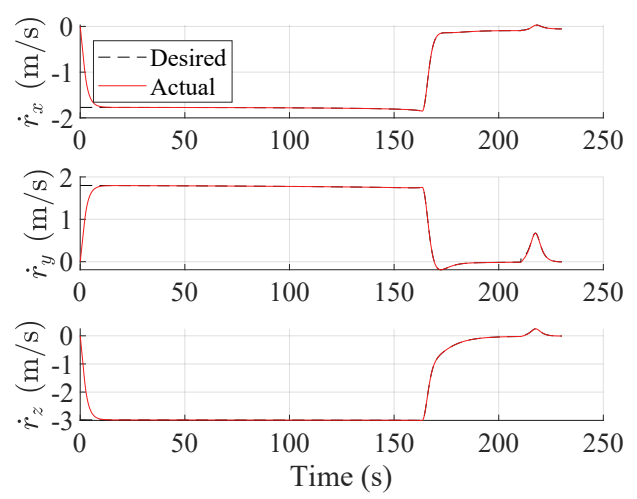

(c) Desired and actual velocity.
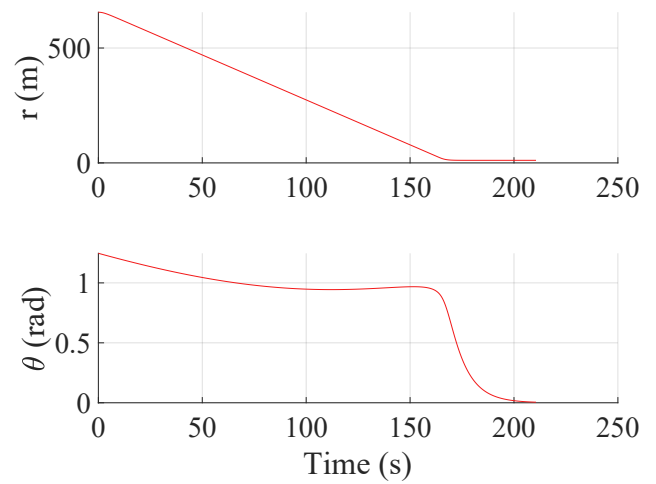

(b) Convergence of $r$ and $\theta$ over time.
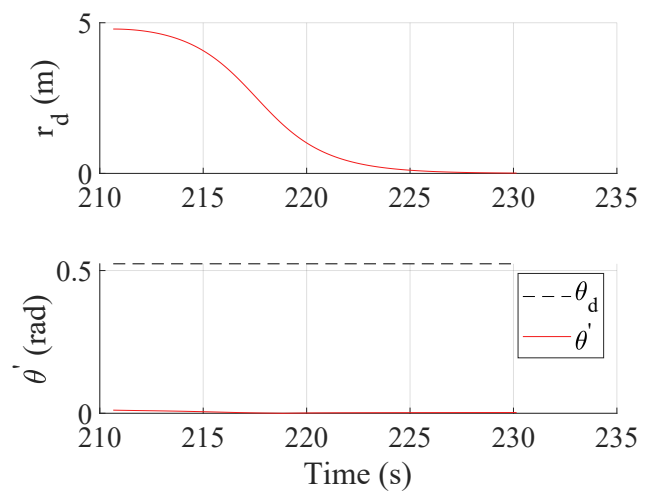

(d) Convergence of $r_{d}$ and $\theta^{\prime}$ over time.

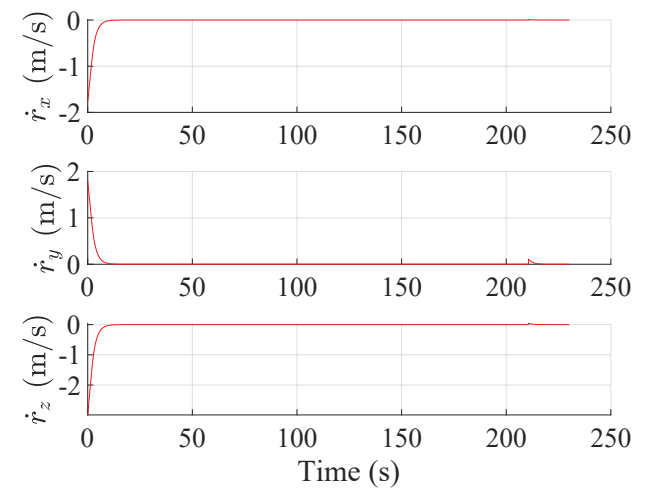

(e) Velocity errors.

Figure 6.1: Acceleration constraint $u_{\max }=1 \mathrm{~m} / \mathrm{s}^{2}$, simulation case 1 . 


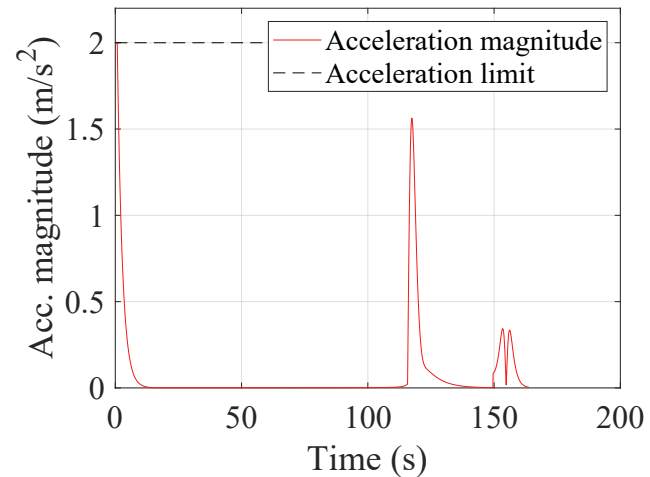

(a) Acceleration commands.
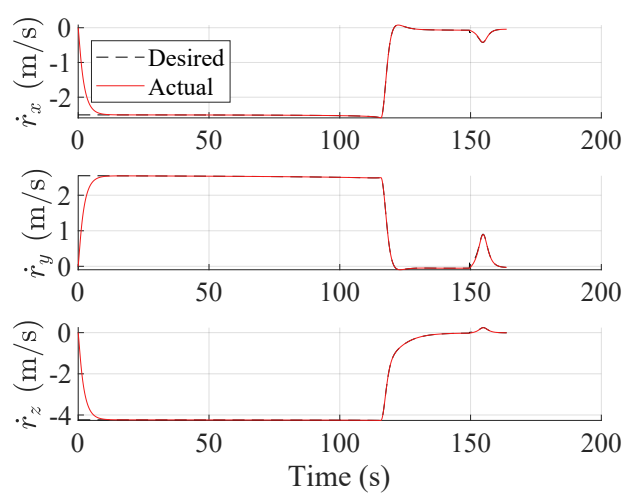

(c) Desired and actual velocity.
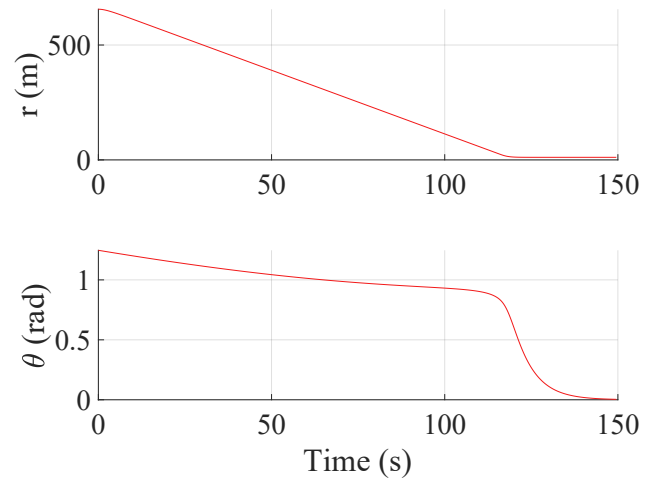

(b) Convergence of $r$ and $\theta$ over time.
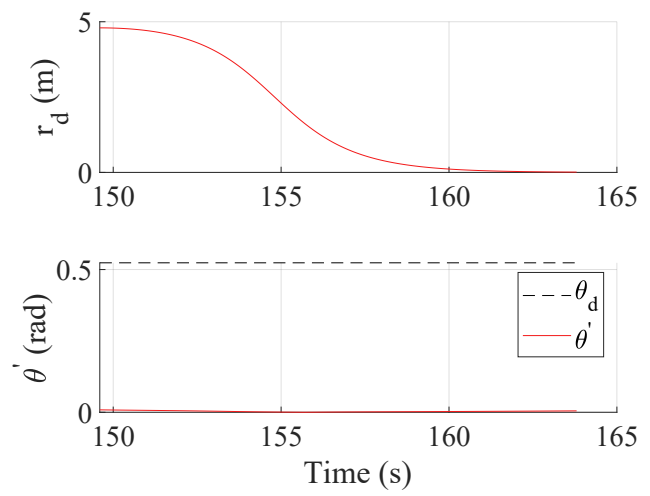

(d) Convergence of $r_{d}$ and $\theta^{\prime}$ over time.
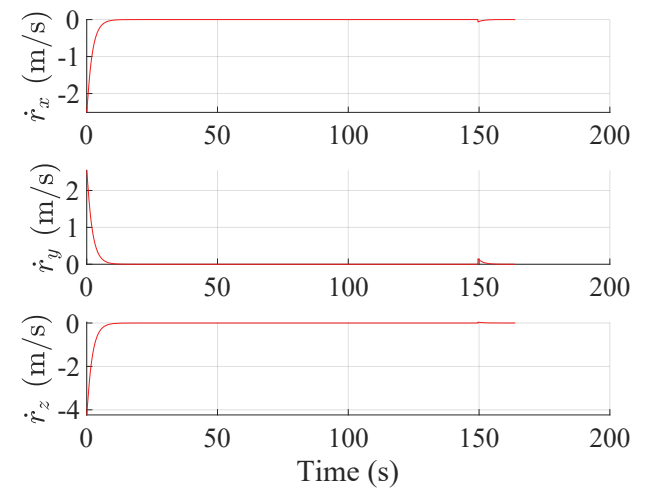

(e) Velocity errors.

Figure 6.2: Acceleration constraint $u_{\max }=2 \mathrm{~m} / \mathrm{s}^{2}$, simulation case 1 . 


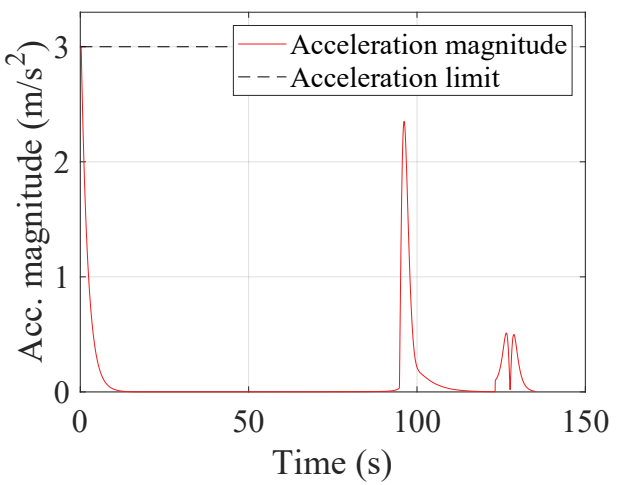

(a) Acceleration commands.
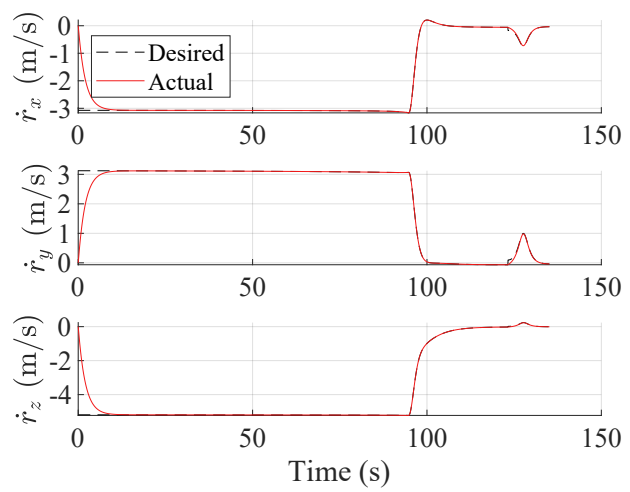

(c) Desired and actual velocity.
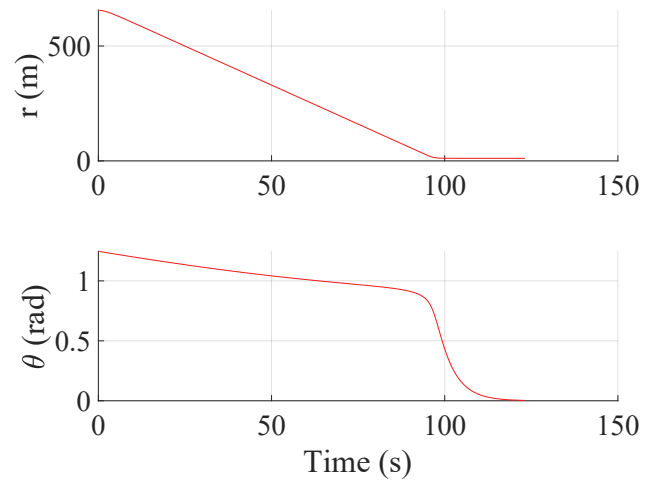

(b) Convergence of $r$ and $\theta$ over time.
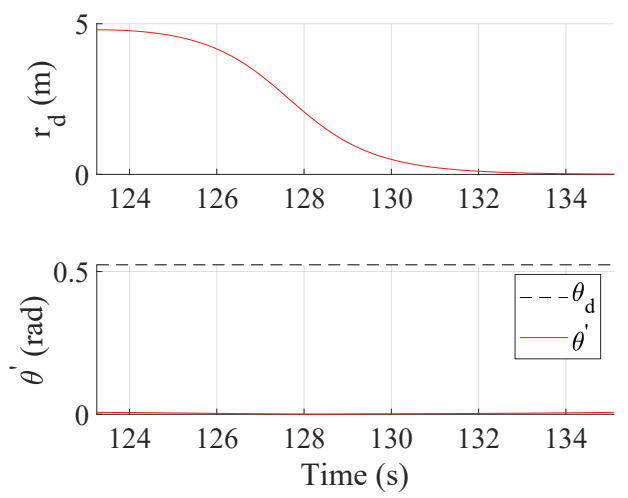

(d) Convergence of $r_{d}$ and $\theta^{\prime}$ over time.
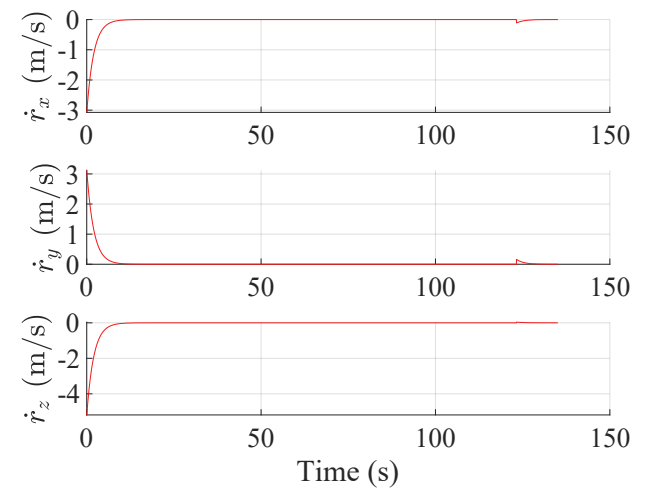

(e) Velocity errors.

Figure 6.3: Acceleration constraint $u_{\max }=3 \mathrm{~m} / \mathrm{s}^{2}$, simulation case 1 . 


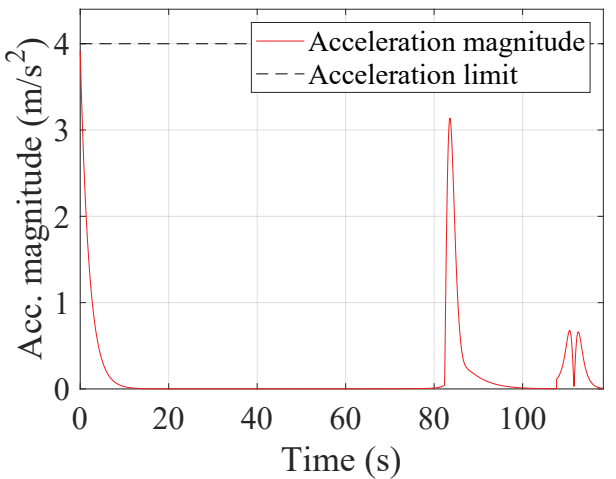

(a) Acceleration commands.

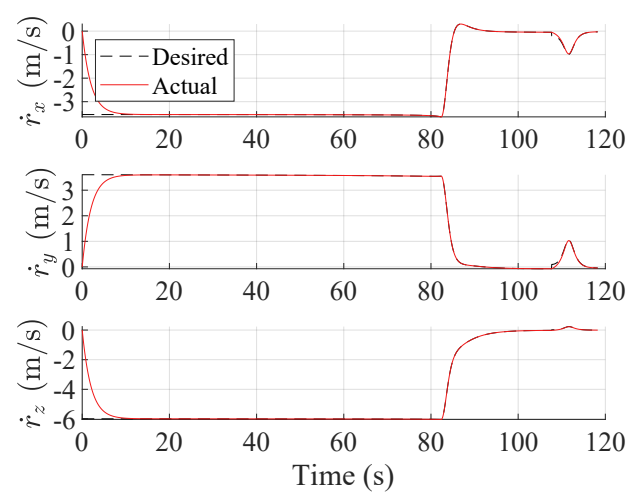

(c) Desired and actual velocity.
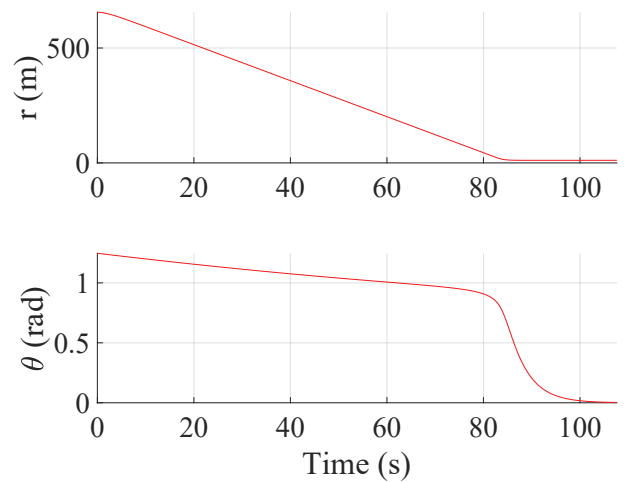

(b) Convergence of $r$ and $\theta$ over time.
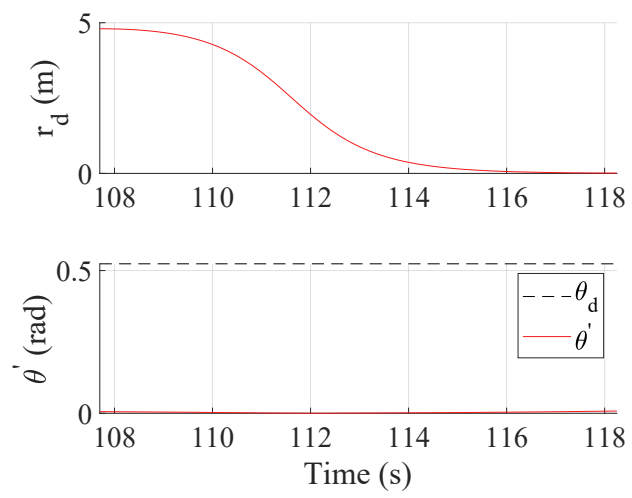

(d) Convergence of $r_{d}$ and $\theta^{\prime}$ over time.

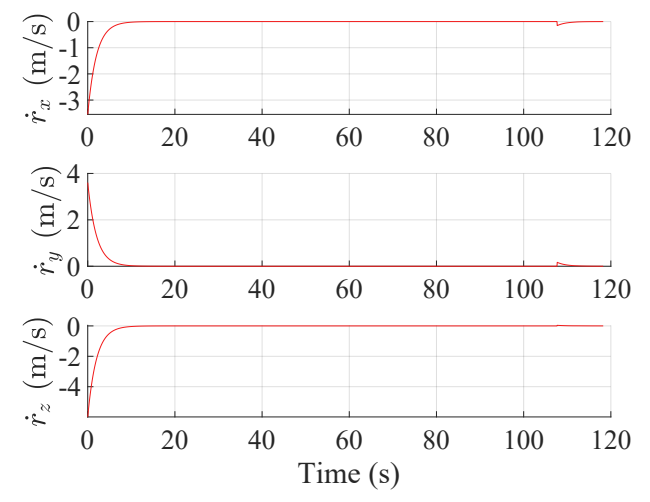

(e) Velocity errors.

Figure 6.4: Acceleration constraint $u_{\max }=4 \mathrm{~m} / \mathrm{s}^{2}$, simulation case 1 . 


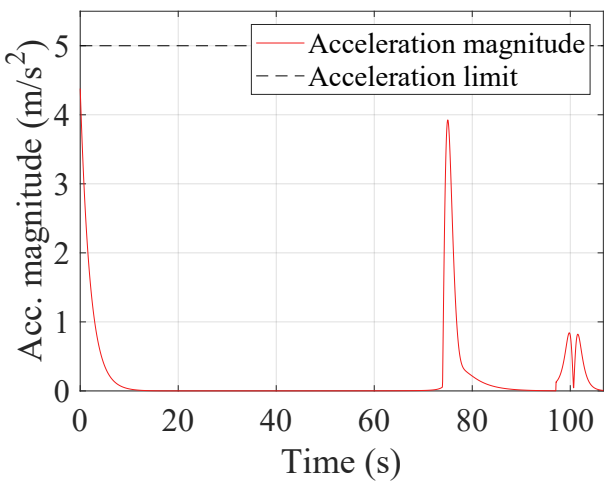

(a) Acceleration commands.

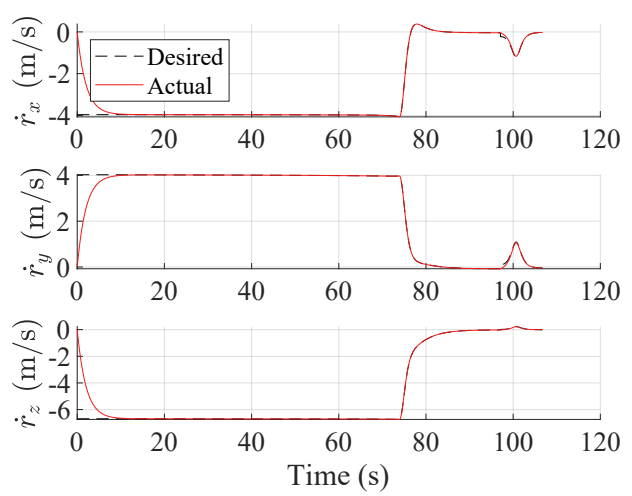

(c) Desired and actual velocity.
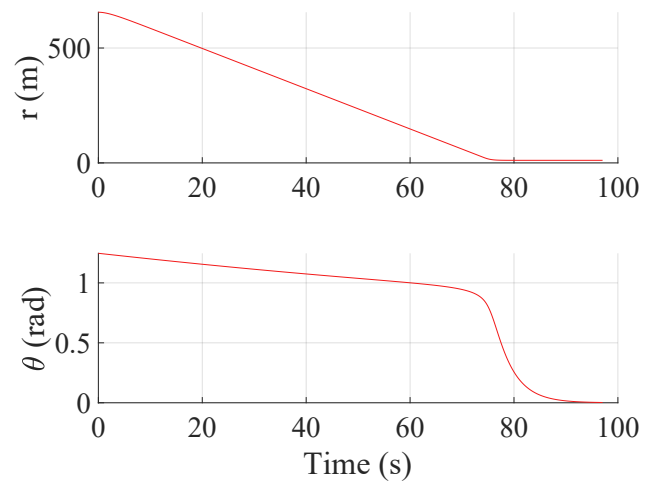

(b) Convergence of $r$ and $\theta$ over time.
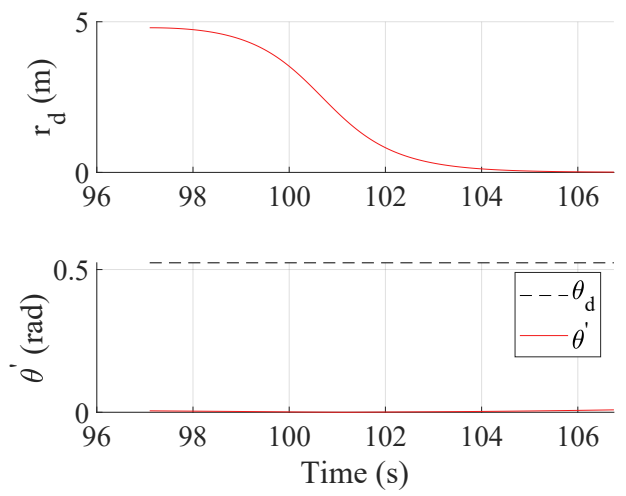

(d) Convergence of $r_{d}$ and $\theta^{\prime}$ over time.
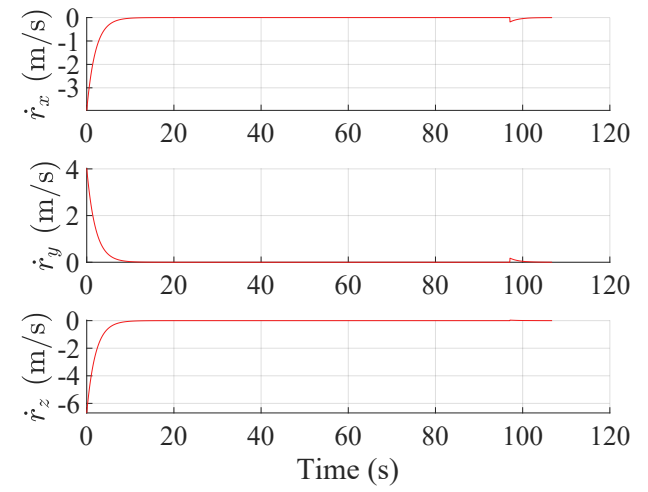

(e) Velocity errors.

Figure 6.5: Acceleration constraint $u_{\max }=5 \mathrm{~m} / \mathrm{s}^{2}$, simulation case 1 . 


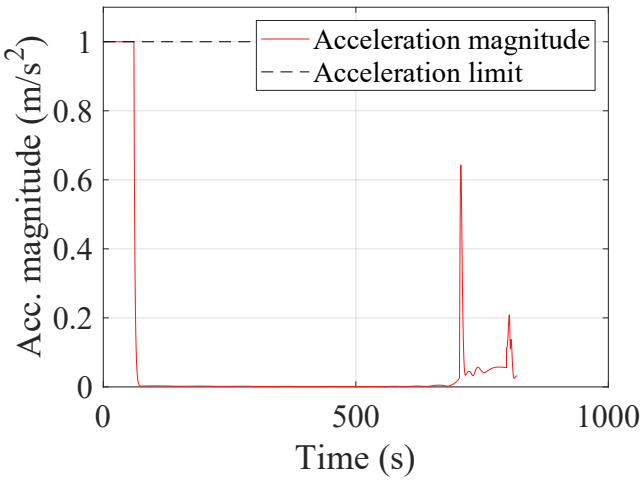

(a) Acceleration commands.
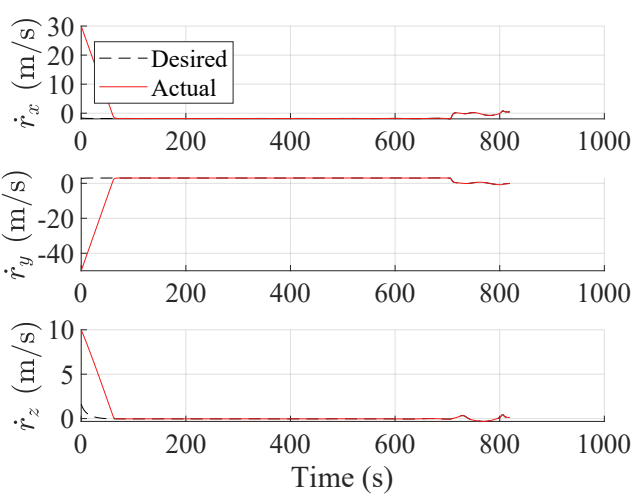

(c) Desired and actual velocity.
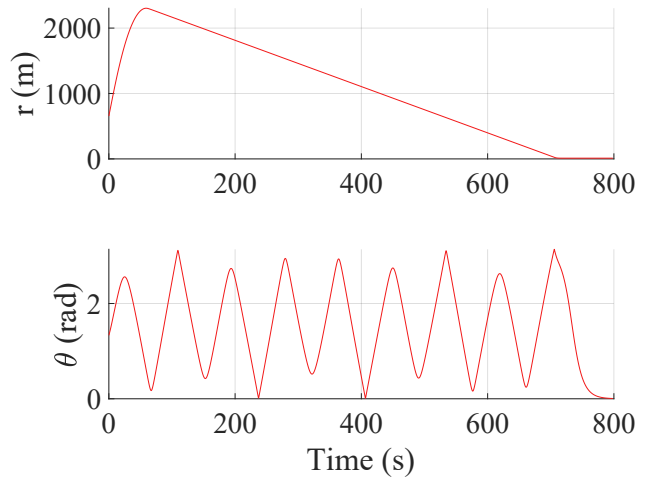

(b) Convergence of $r$ and $\theta$ over time.
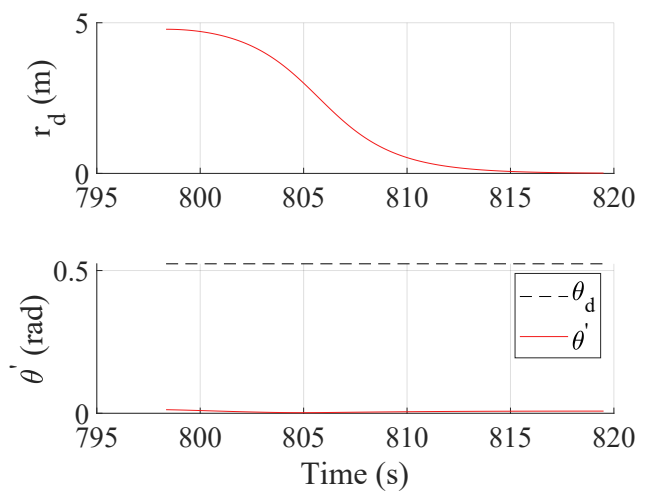

(d) Convergence of $r_{d}$ and $\theta^{\prime}$ over time.

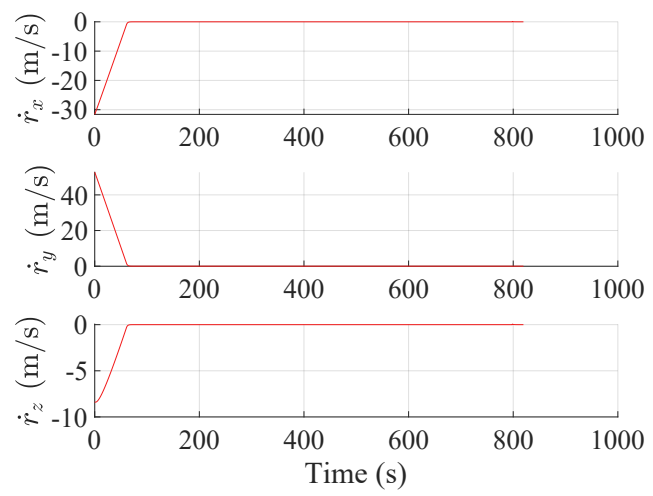

(e) Velocity errors.

Figure 6.6: Acceleration constraint $u_{\max }=1 \mathrm{~m} / \mathrm{s}^{2}$, simulation case 4 . 


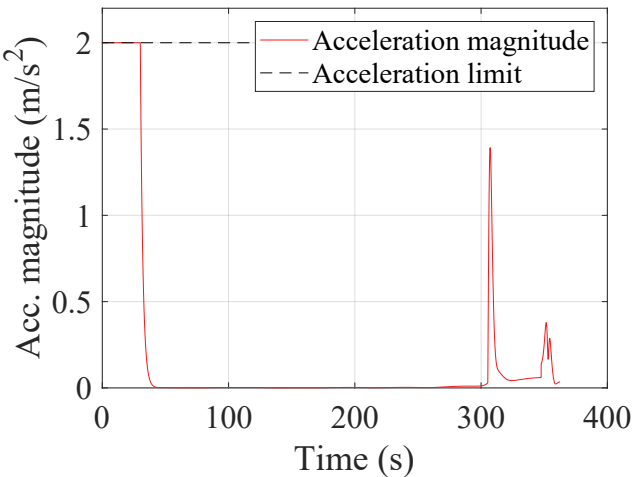

(a) Acceleration commands.
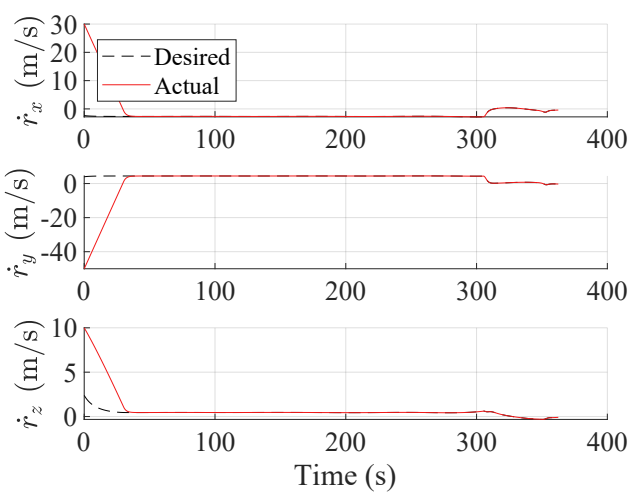

(c) Desired and actual velocity.
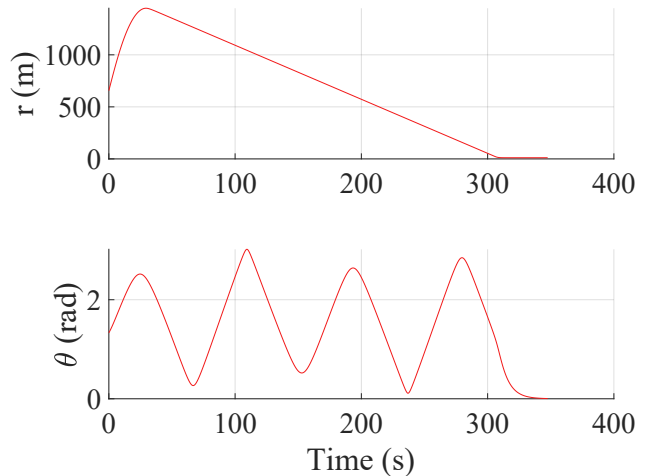

(b) Convergence of $r$ and $\theta$ over time.
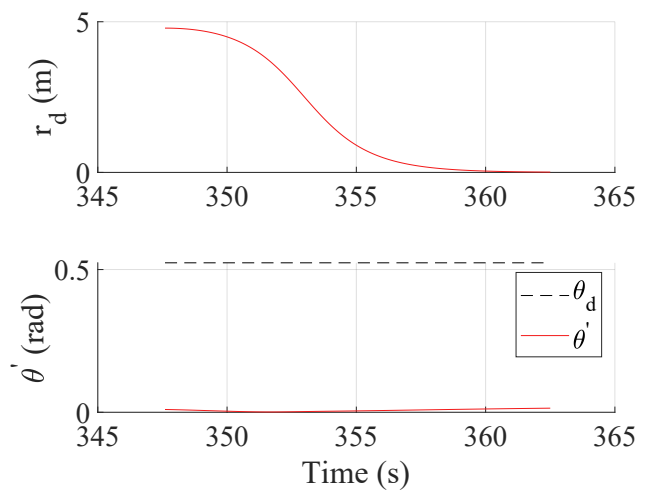

(d) Convergence of $r_{d}$ and $\theta^{\prime}$ over time.
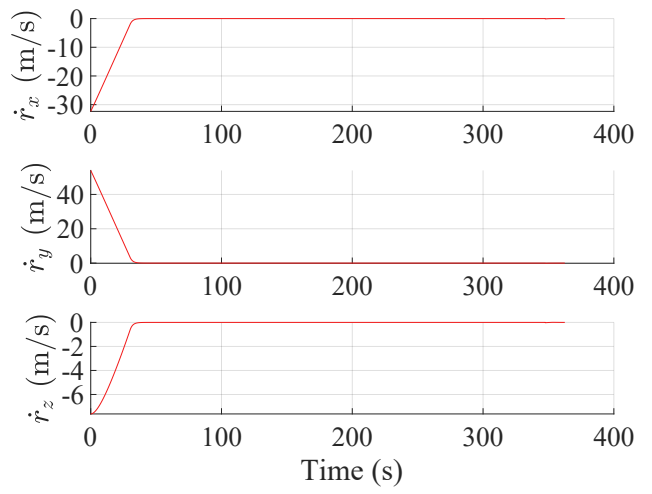

(e) Velocity errors.

Figure 6.7: Acceleration constraint $u_{\max }=2 \mathrm{~m} / \mathrm{s}^{2}$, simulation case 4 . 


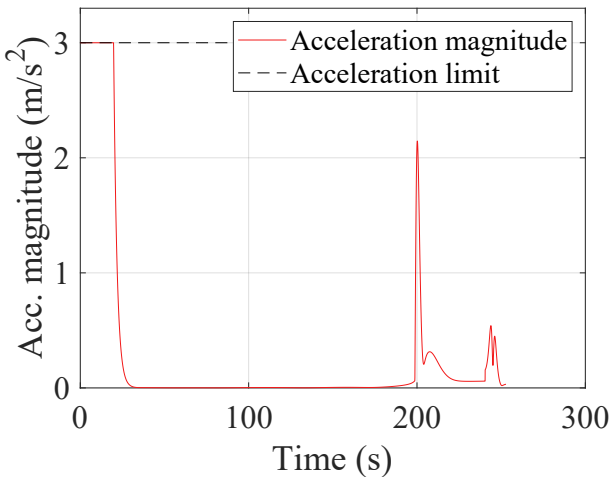

(a) Acceleration commands.
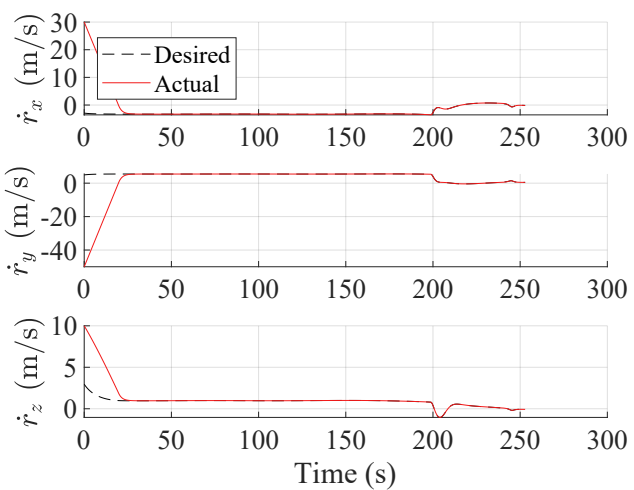

(c) Desired and actual velocity.
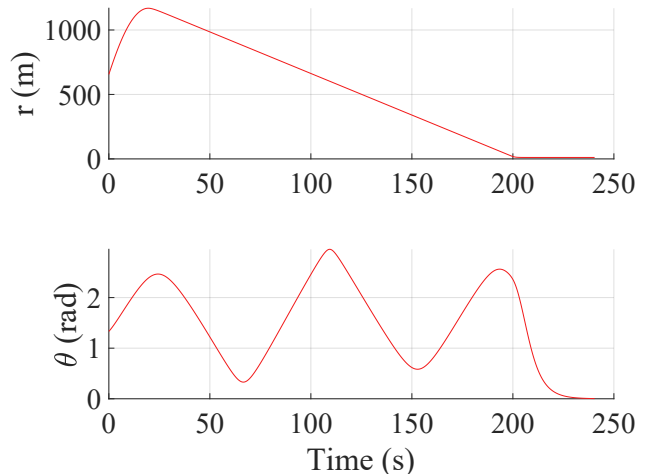

(b) Convergence of $r$ and $\theta$ over time.
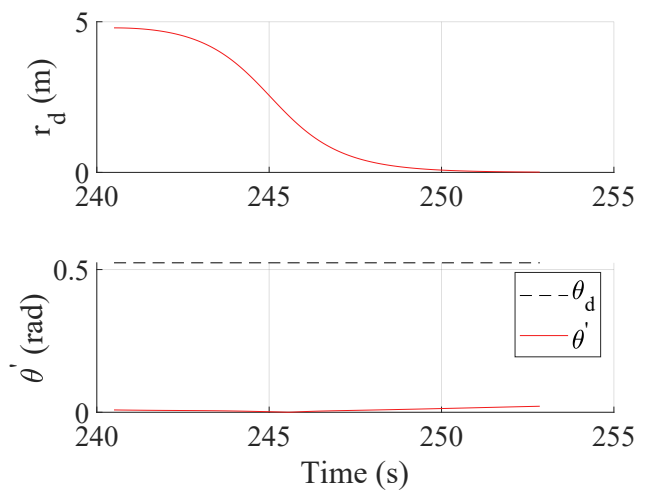

(d) Convergence of $r_{d}$ and $\theta^{\prime}$ over time.

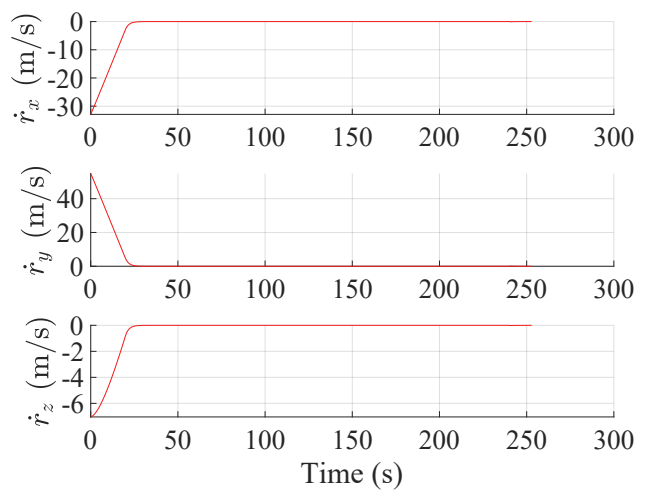

(e) Velocity errors.

Figure 6.8: Acceleration constraint $u_{\max }=3 \mathrm{~m} / \mathrm{s}^{2}$, simulation case 4 . 


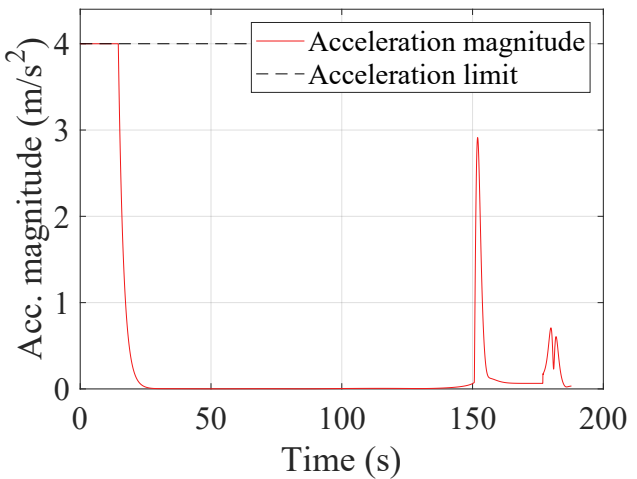

(a) Acceleration commands.
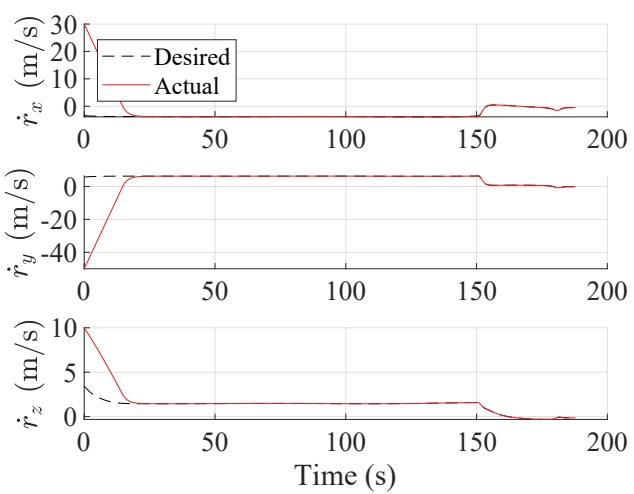

(c) Desired and actual velocity.
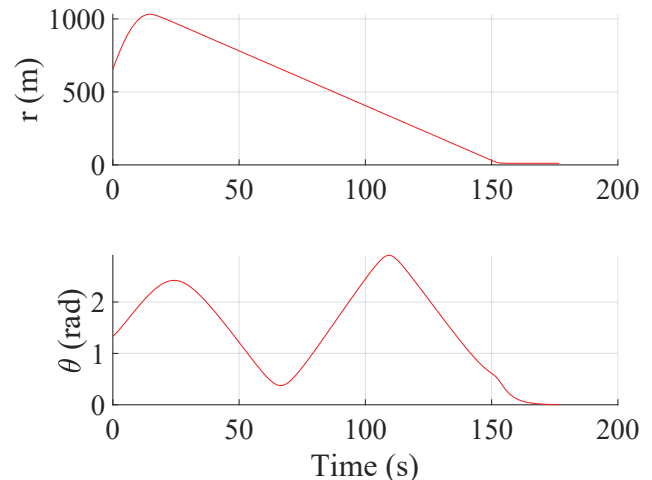

(b) Convergence of $r$ and $\theta$ over time.
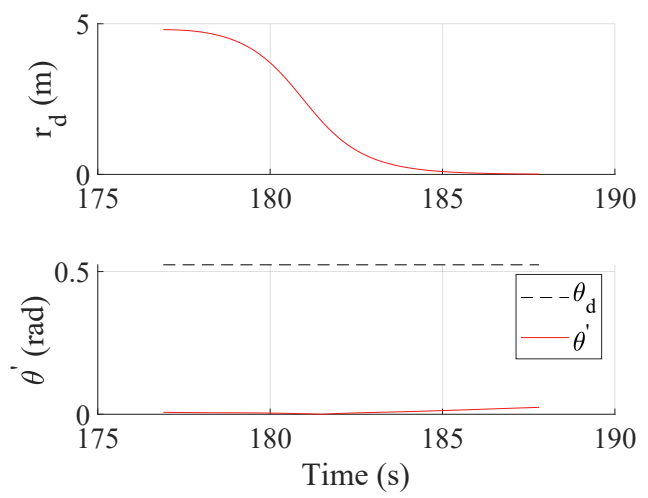

(d) Convergence of $r_{d}$ and $\theta^{\prime}$ over time.
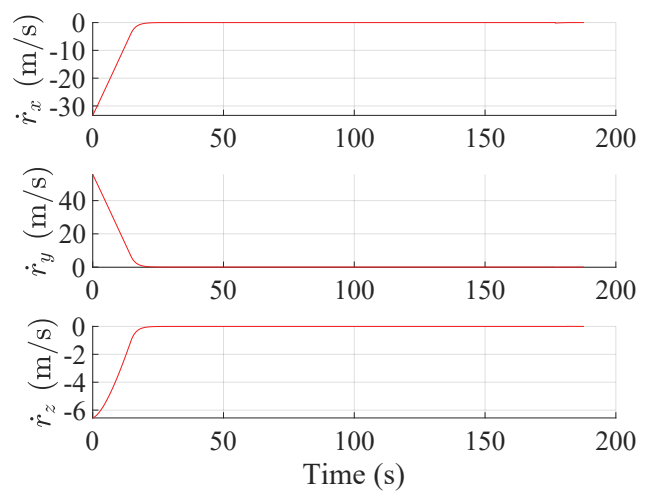

(e) Velocity errors.

Figure 6.9: Acceleration constraint $u_{\max }=4 \mathrm{~m} / \mathrm{s}^{2}$, simulation case 4 . 


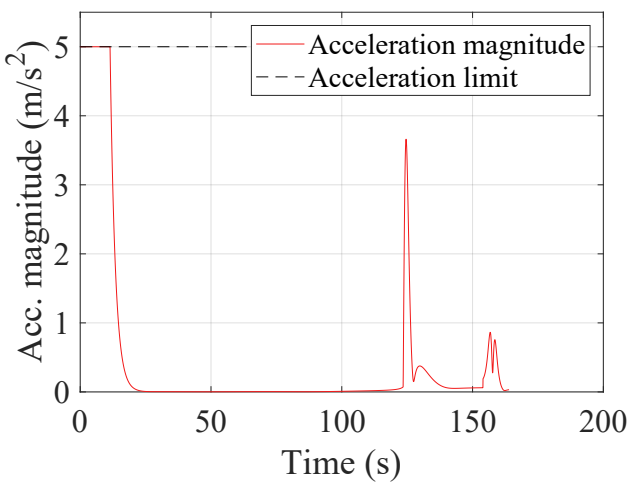

(a) Acceleration commands.

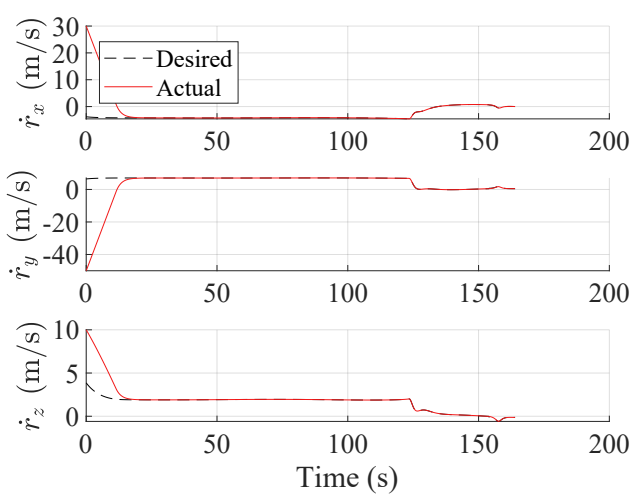

(c) Desired and actual velocity.
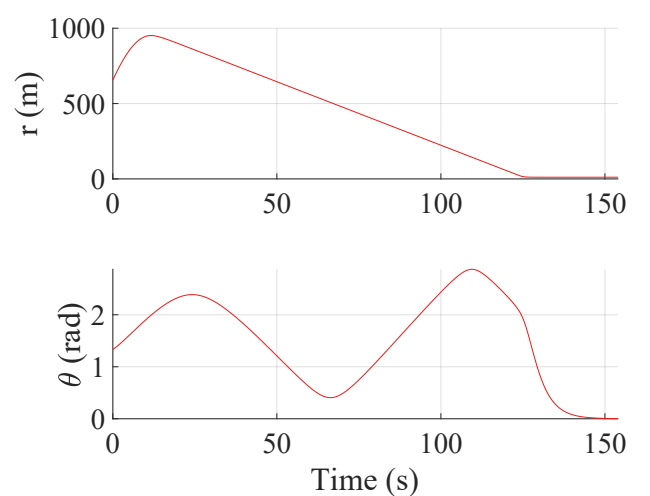

(b) Convergence of $r$ and $\theta$ over time.
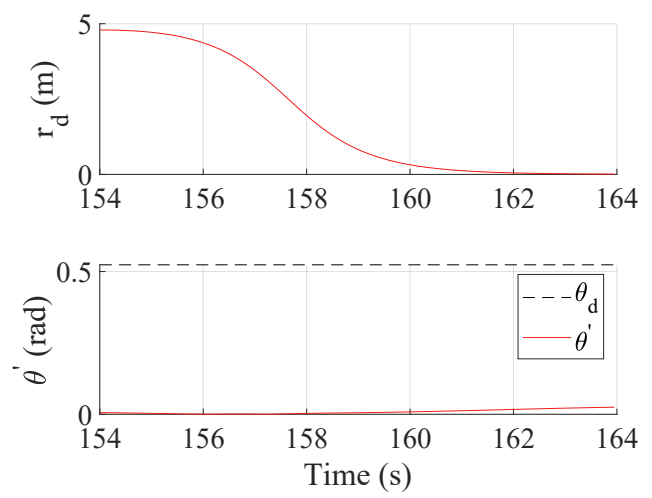

(d) Convergence of $r_{d}$ and $\theta^{\prime}$ over time.

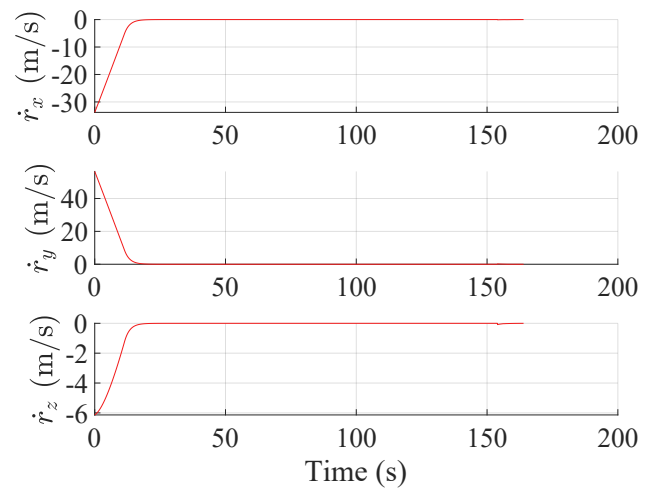

(e) Velocity errors.

Figure 6.10: Acceleration constraint $u_{\max }=5 \mathrm{~m} / \mathrm{s}^{2}$, simulation case 4 . 


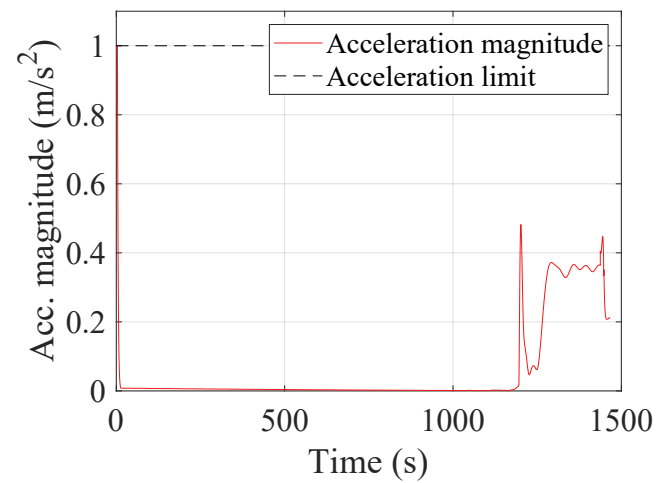

(a) Acceleration commands.

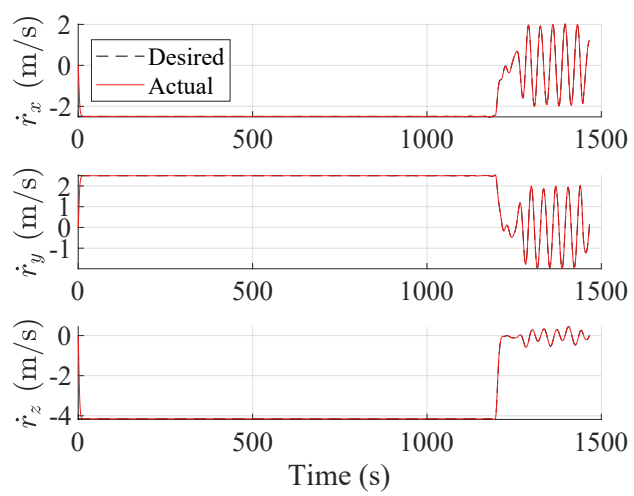

(c) Desired and actual velocity.
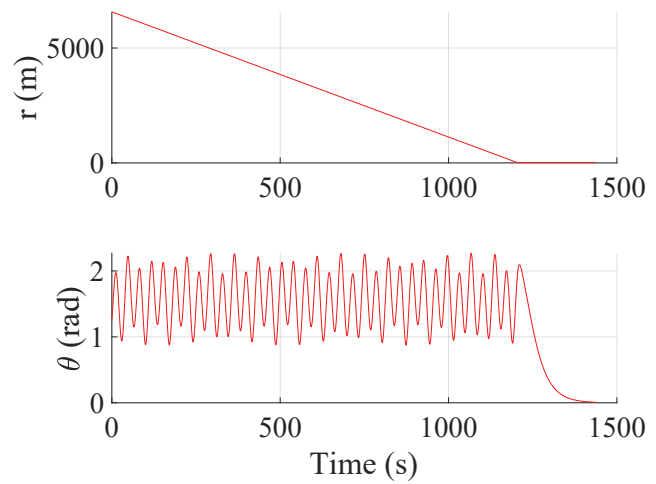

(b) Convergence of $r$ and $\theta$ over time.
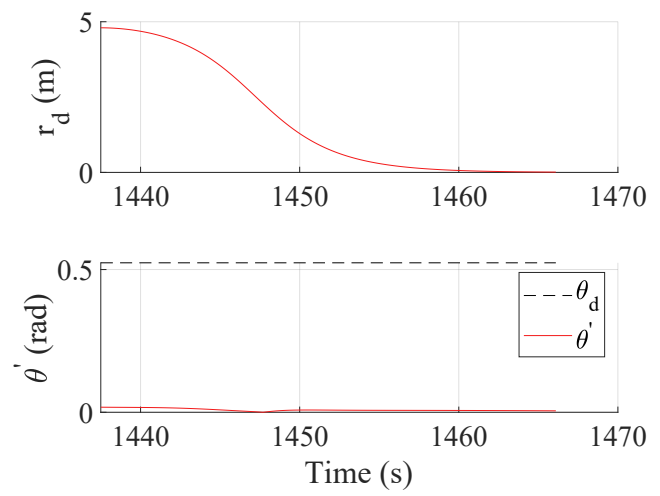

(d) Convergence of $r_{d}$ and $\theta^{\prime}$ over time.

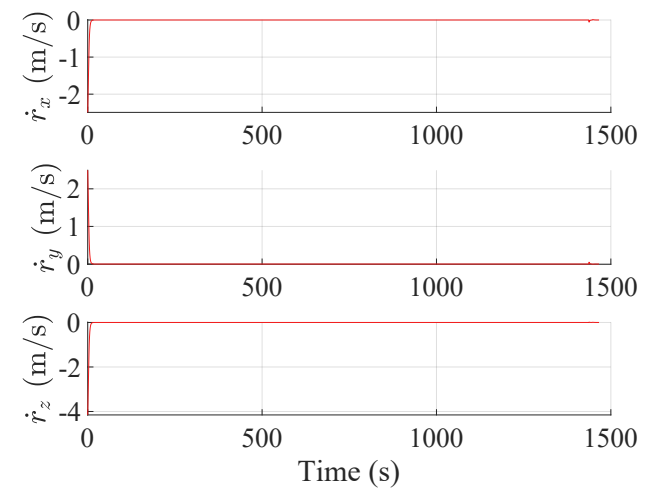

(e) Velocity errors.

Figure 6.11: Acceleration constraint $u_{\max }=1 \mathrm{~m} / \mathrm{s}^{2}$, simulation case 7 . 


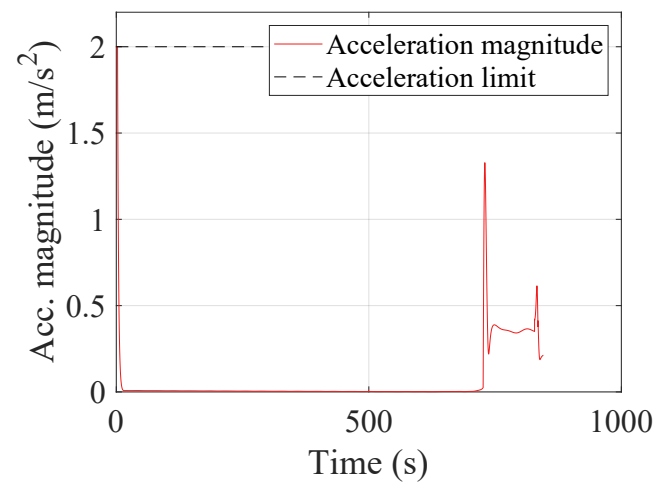

(a) Acceleration commands.

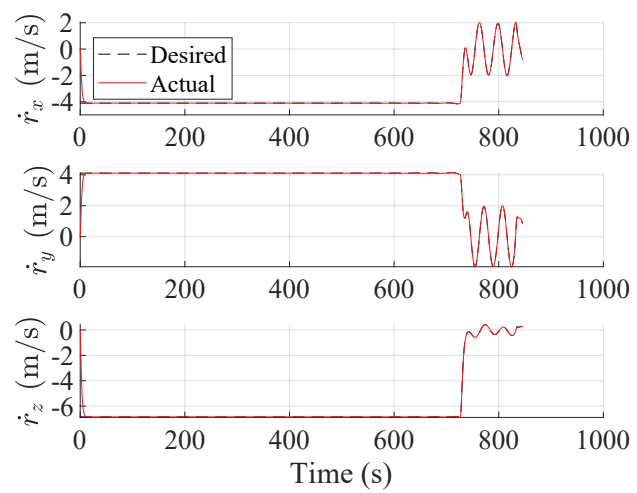

(c) Desired and actual velocity.
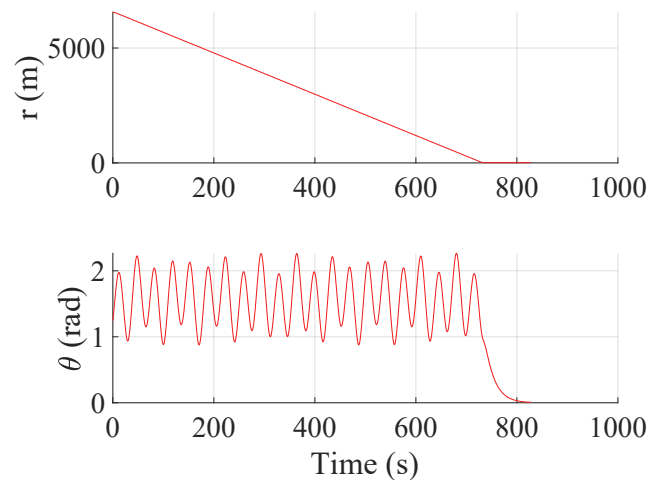

(b) Convergence of $r$ and $\theta$ over time.
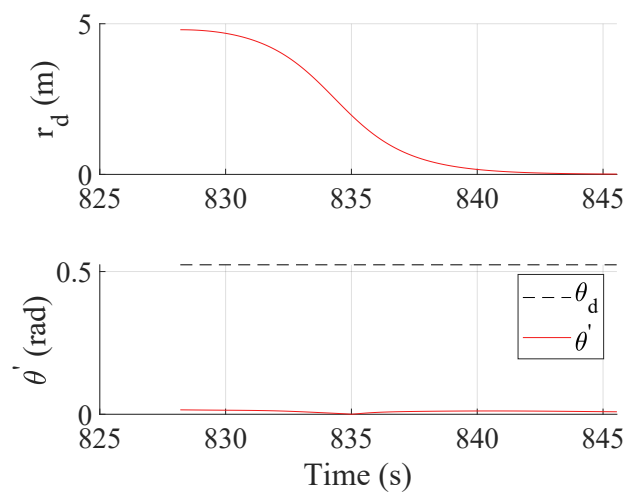

(d) Convergence of $r_{d}$ and $\theta^{\prime}$ over time.

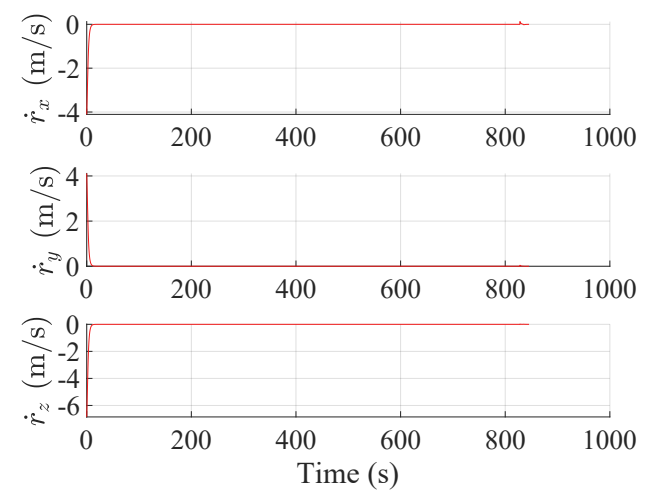

(e) Velocity errors.

Figure 6.12: Acceleration constraint $u_{\max }=2 \mathrm{~m} / \mathrm{s}^{2}$, simulation case 7 . 


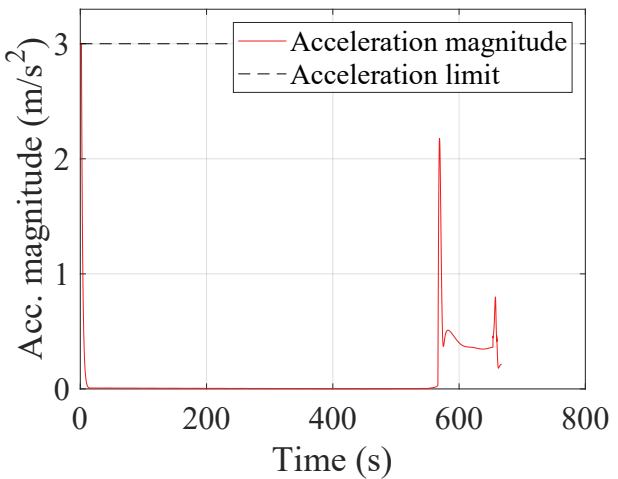

(a) Acceleration commands.

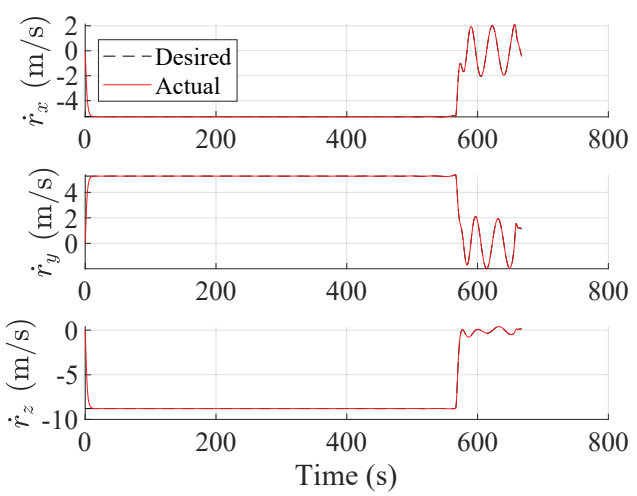

(c) Desired and actual velocity.
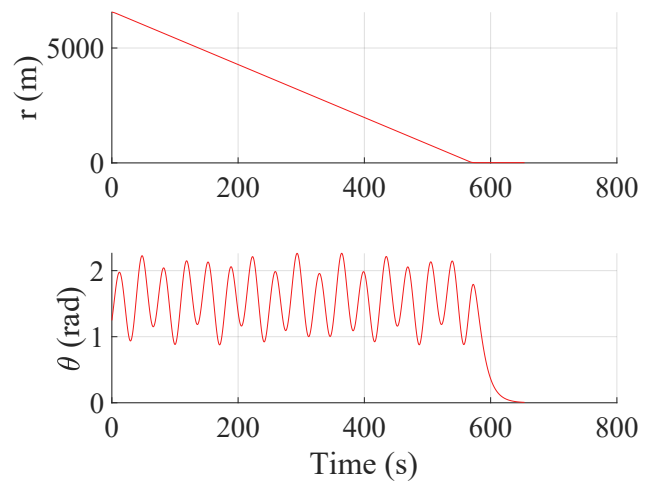

(b) Convergence of $r$ and $\theta$ over time.
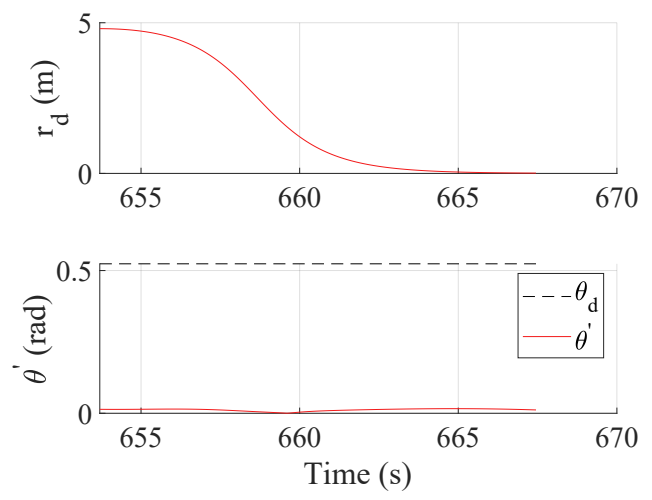

(d) Convergence of $r_{d}$ and $\theta^{\prime}$ over time.
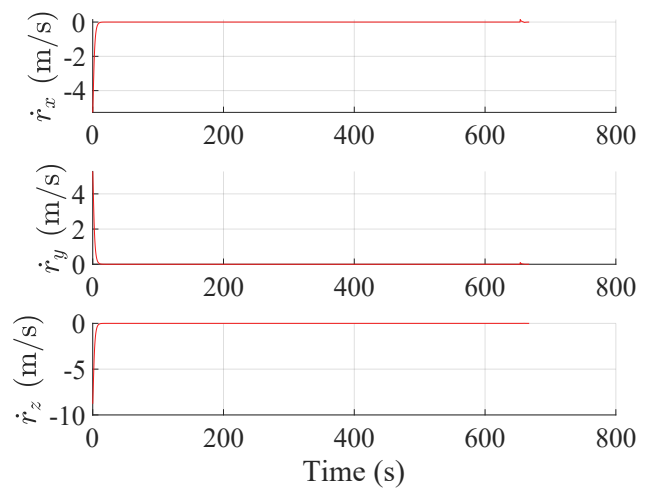

(e) Velocity errors.

Figure 6.13: Acceleration constraint $u_{\max }=3 \mathrm{~m} / \mathrm{s}^{2}$, simulation case 7 . 


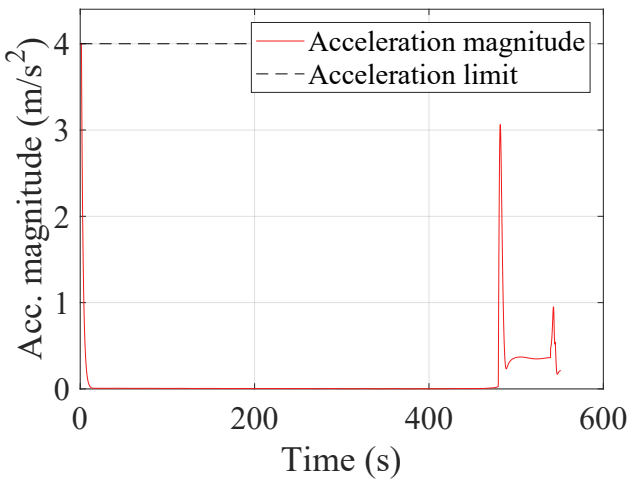

(a) Acceleration commands.

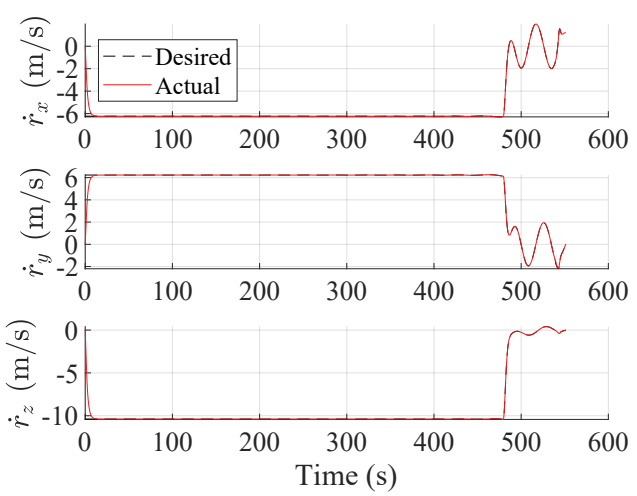

(c) Desired and actual velocity.
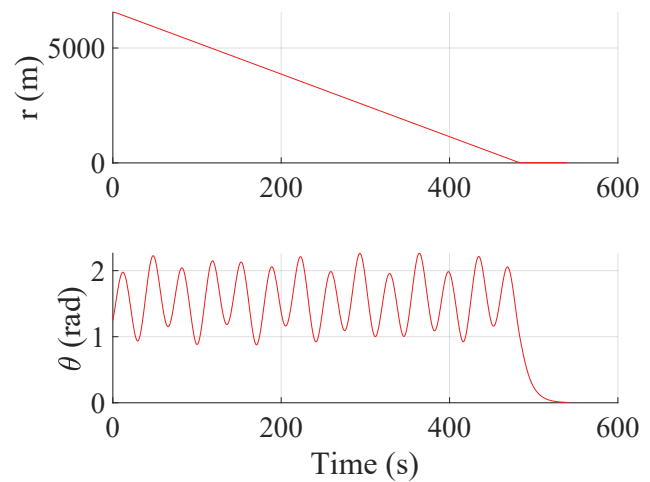

(b) Convergence of $r$ and $\theta$ over time.
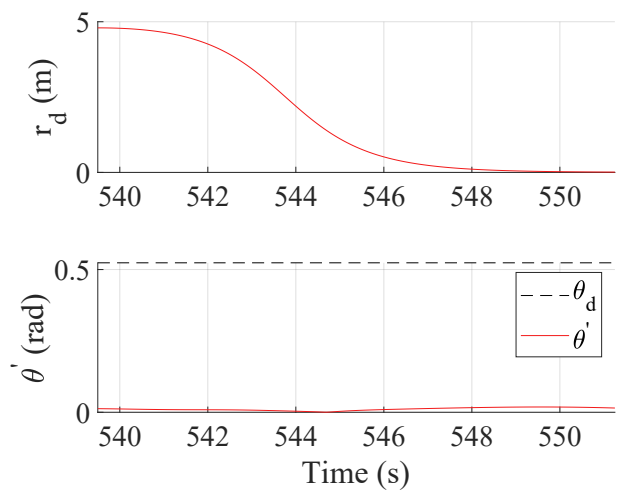

(d) Convergence of $r_{d}$ and $\theta^{\prime}$ over time.

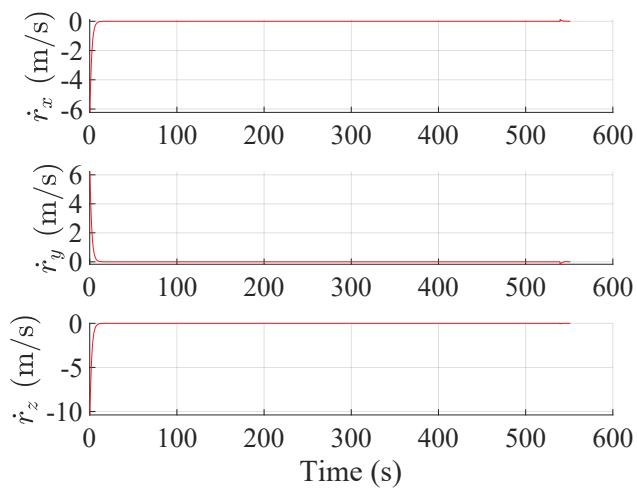

(e) Velocity errors.

Figure 6.14: Acceleration constraint $u_{\max }=4 \mathrm{~m} / \mathrm{s}^{2}$, simulation case 7 . 


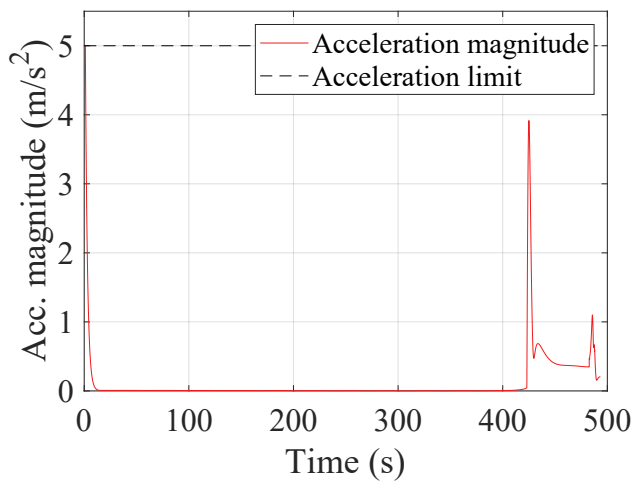

(a) Acceleration commands.

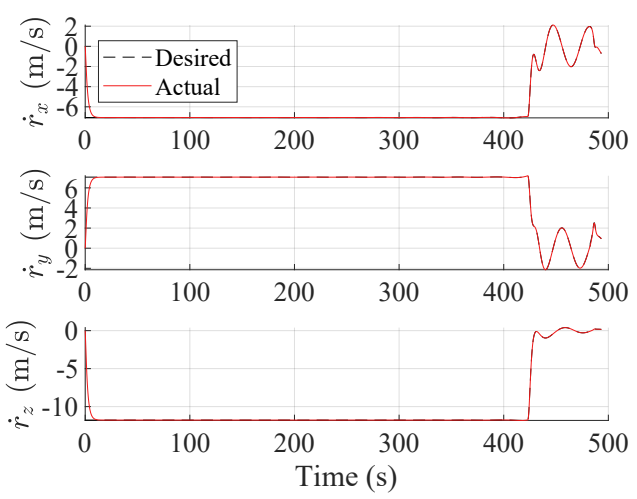

(c) Desired and actual velocity.
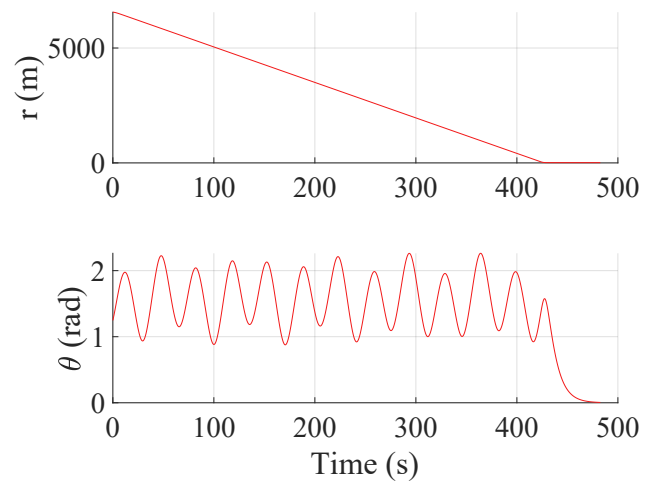

(b) Convergence of $r$ and $\theta$ over time.
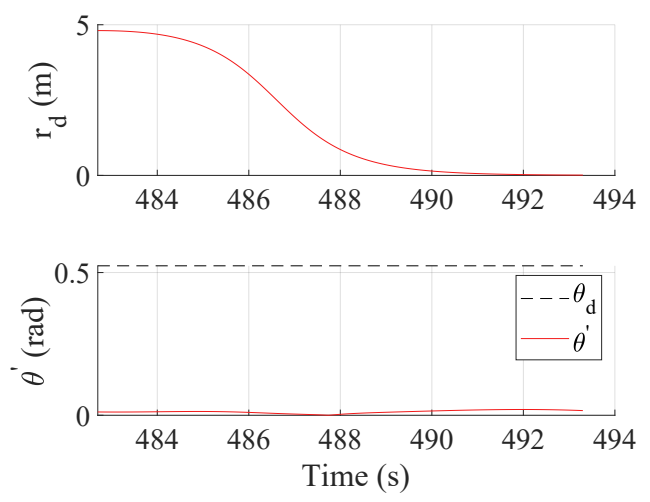

(d) Convergence of $r_{d}$ and $\theta^{\prime}$ over time.
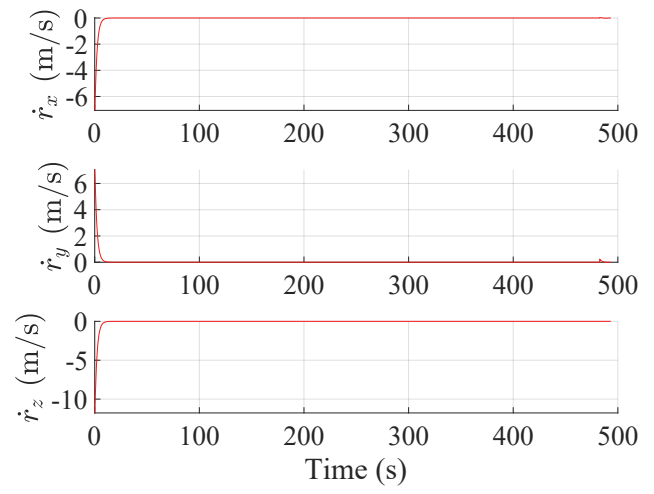

(e) Velocity errors.

Figure 6.15: Acceleration constraint $u_{\max }=5 \mathrm{~m} / \mathrm{s}^{2}$, simulation case 7 . 


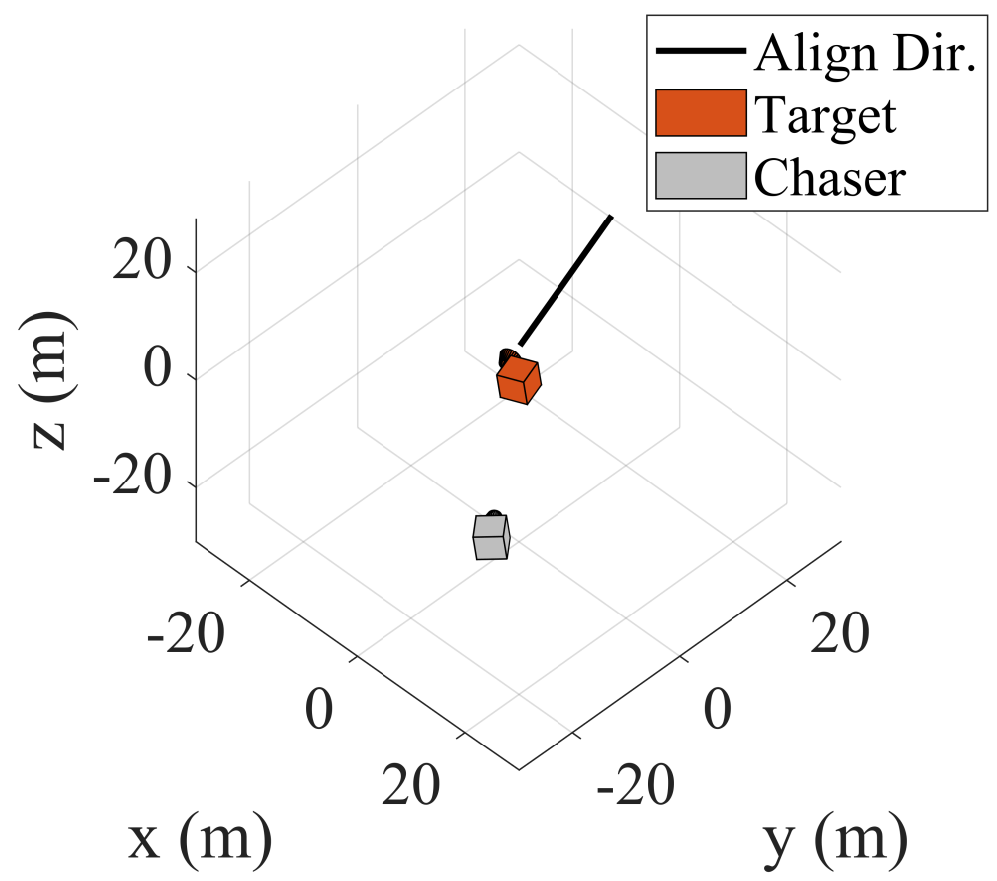

(a) $t=704.05 \mathrm{~s}$.

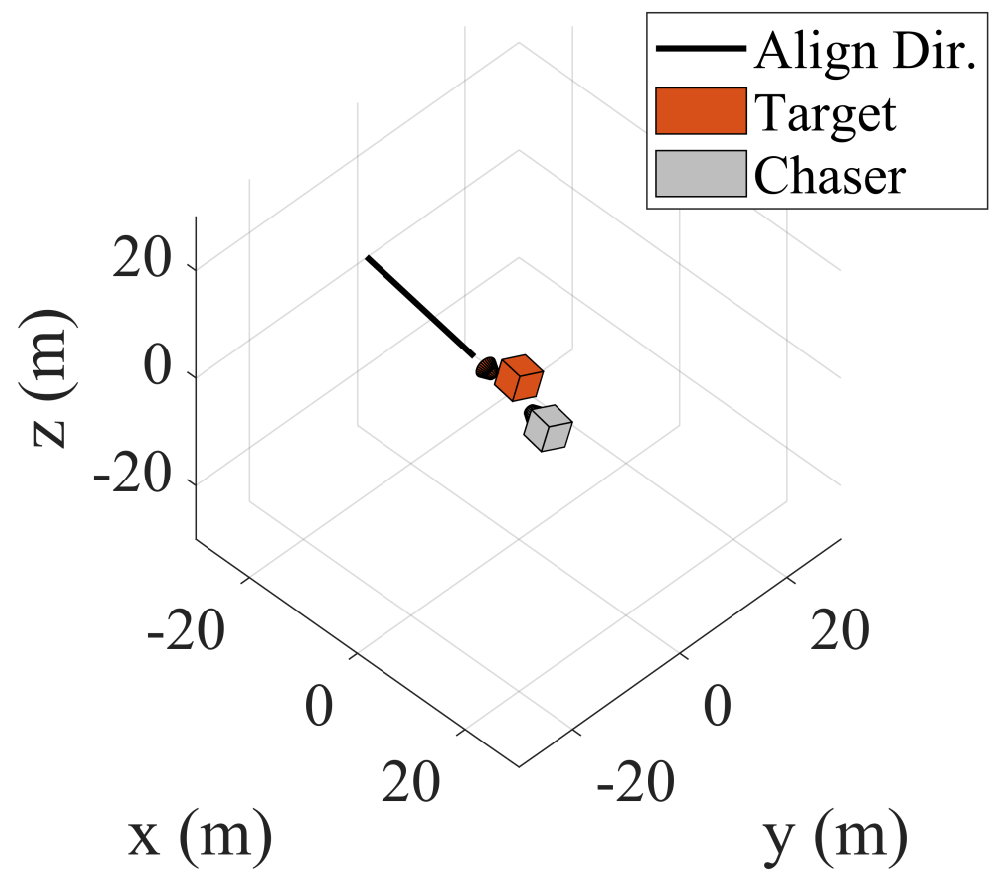

(b) $t=719.75 \mathrm{~s}$. 

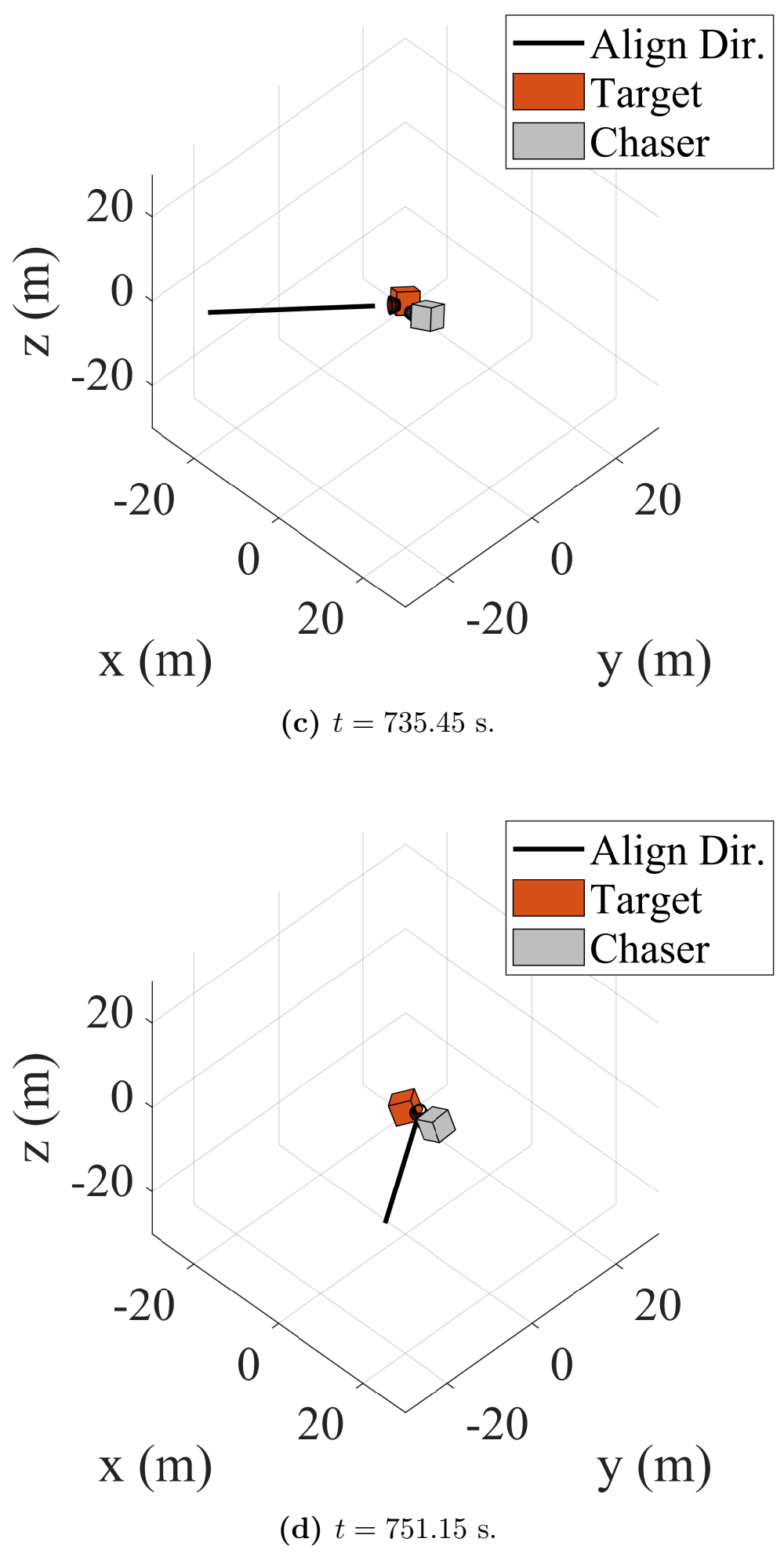


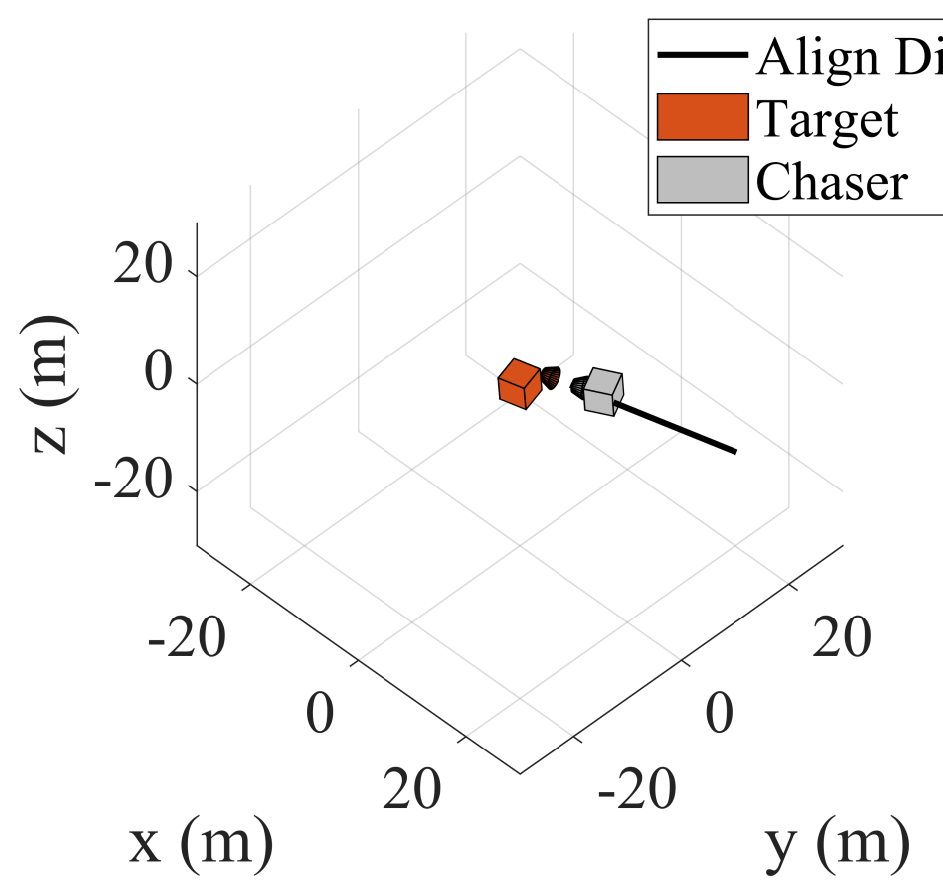

(e) $t=766.85 \mathrm{~s}$.

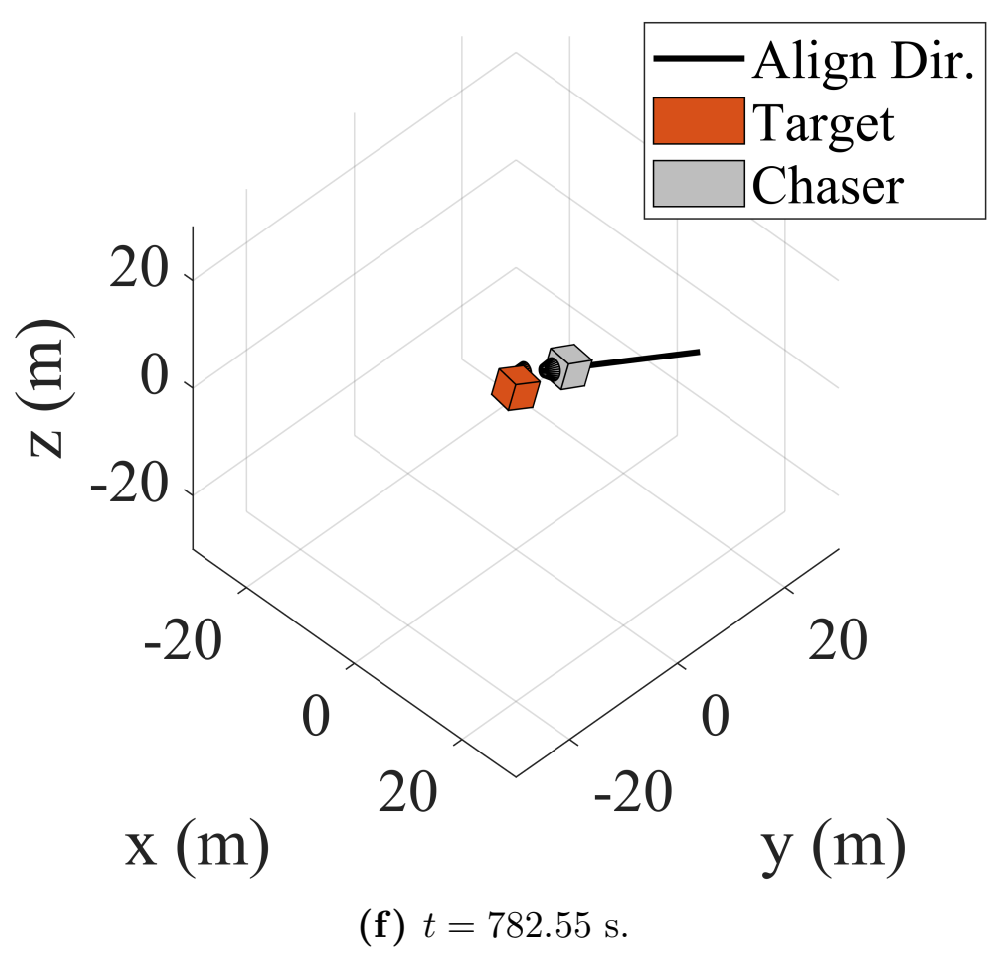




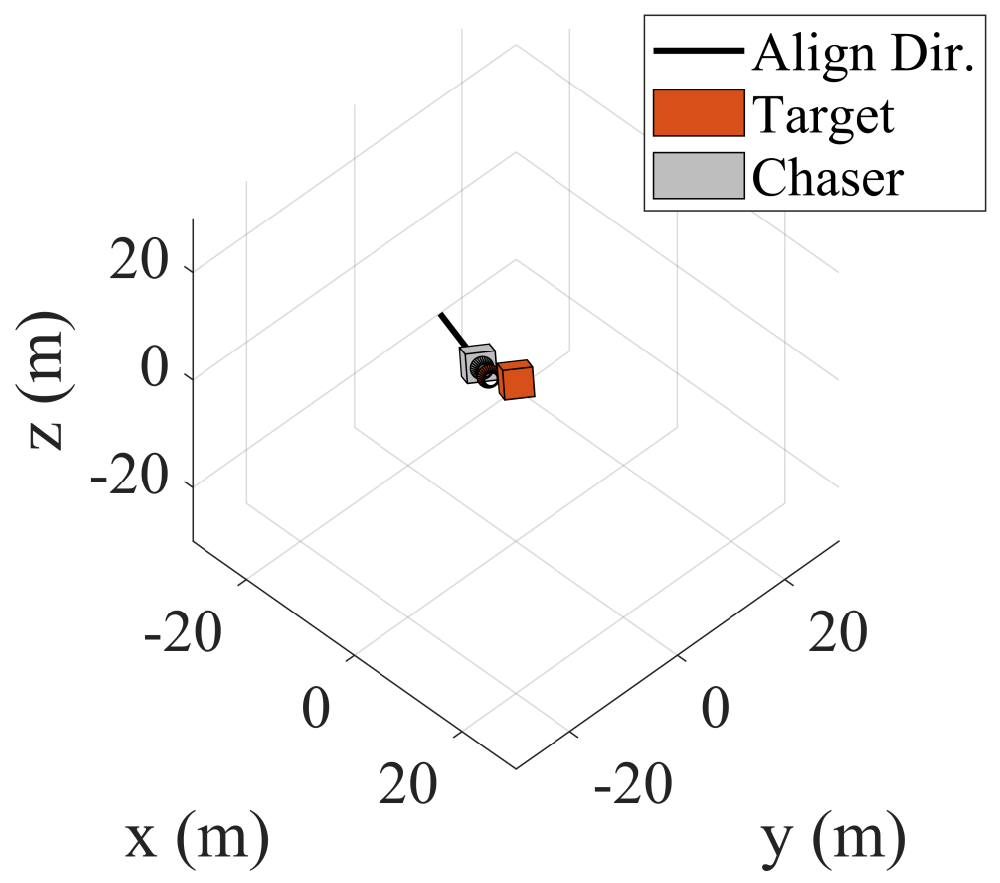

(g) $t=798.25 \mathrm{~s}$.

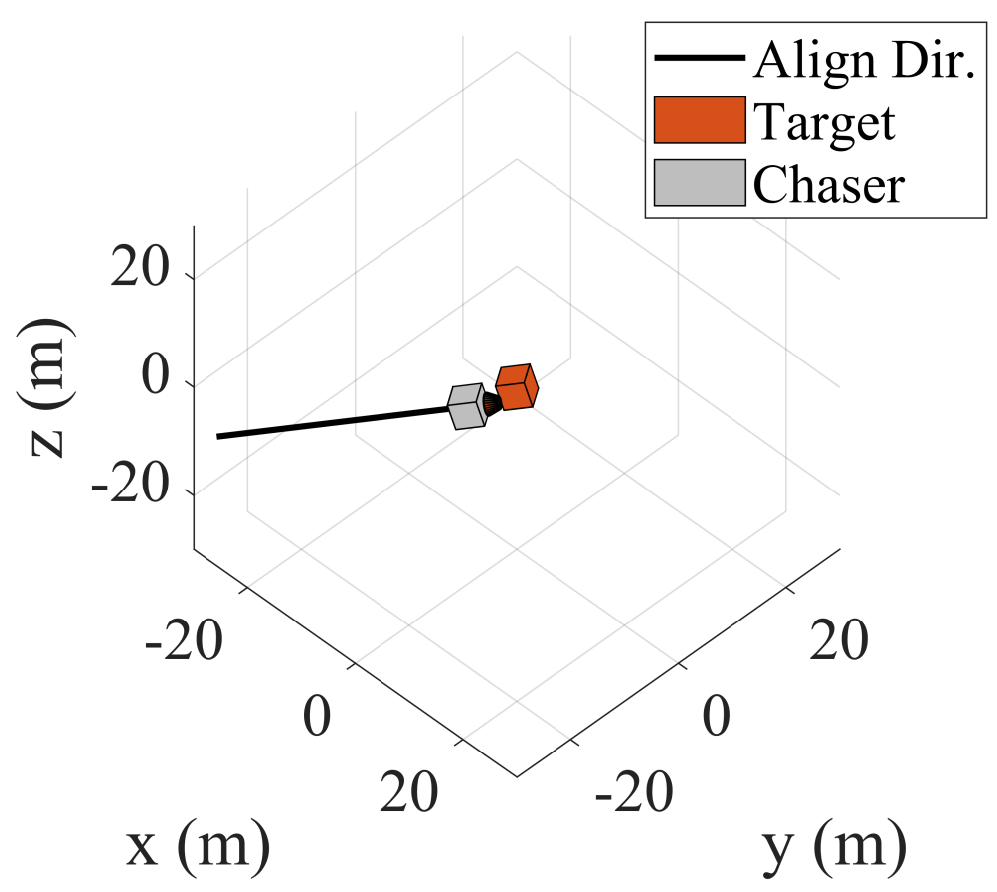

(h) $t=814.00 \mathrm{~s}$.

Figure 6.16: Time-lapse of three-dimensional docking simulation. 
To illustrate the performance when the target spin is relatively low, let us consider the initial condition cases 1 and 2. Figure 6.17 shows the acceleration profiles for initial condition case 1 when $u_{\max }=5.0 \mathrm{~m} / \mathrm{s}^{2}$ and the weight set is varied. Likewise, Fig. 6.18 shows the acceleration profiles for initial condition case 2 for $u_{\max }=3.0 \mathrm{~m} / \mathrm{s}^{2}$ as the weight set is varied. Clearly, when time is more heavily penalized, the algorithm selects field parameters which result is a strategy of quickly accelerating to maximum speed, coasting, and then quickly decelerating to achieve the docking maneuver in minimum time. As the fuel becomes more penalized, the optimization selects parameters which result in progressively smaller impulses with increasingly long coast times.

It should be noted that simulation cases 1 and 2 are quite representative of many practical debris removal missions. For example, in work by Efimov et al. [36], the long-term attitude dynamics of sun-synchronous orbits (SSO) are studied, which is presently the orbital ring most densely populated with space debris. It was found that due to eddy-currents from the atmosphere, orbital debris in SSO exponentially decrease in their attitude dynamics until their angular velocity is similar in magnitude to the frequency of the orbit.

In what follows, the individual performance characteristics of Phase 1 and Phase 2 under cost function weight variations are discussed, and particular attention is paid to the cases of higher angular velocity and larger weight disparities where the optimization can perform quite poorly.

\section{Observations of the Phase 1 performance variation}

By observing Tables 6.5-6.8, it is clear that there exists a significant trend downwards in general fuel usage as the weights are increased. However, quite interestingly, the fuel usage trends upwards with weight set 5, tabulated in Table 6.9 with the exceptions of simulation cases 1 and 2. Notably, the maneuver time $T_{P 1}$ does not suffer from this sudden reversal of expectations. Rather, the maneuver time quite reliably decreases as the time penalty increases. This is apparent from Tables 6.10-6.14.

This sudden increase in fuel usage can be explained by a loss of correlation between the estimated and actual fuel usage for higher angular velocities. 


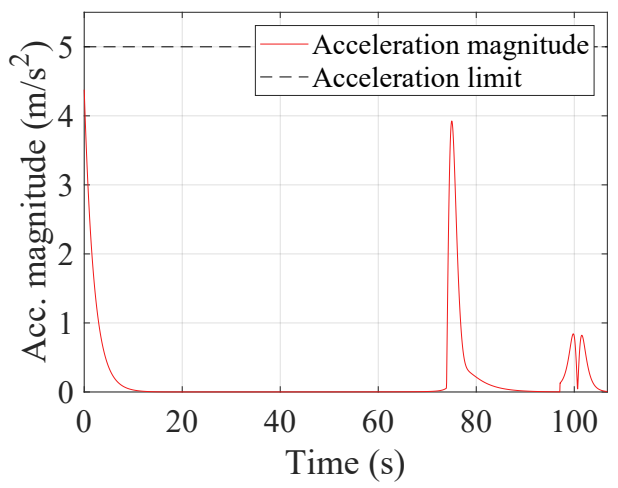

(a) Weight set 1 .

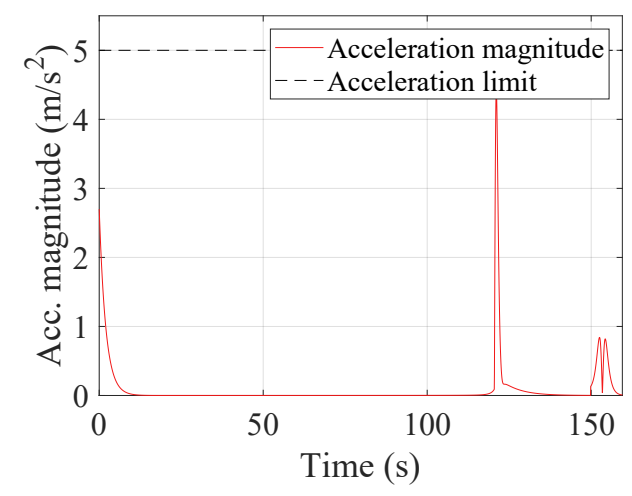

(c) Weight set 3 .

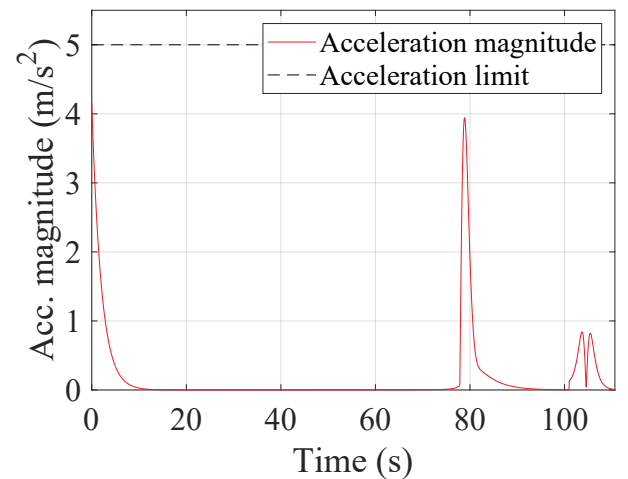

(b) Weight set 2 .

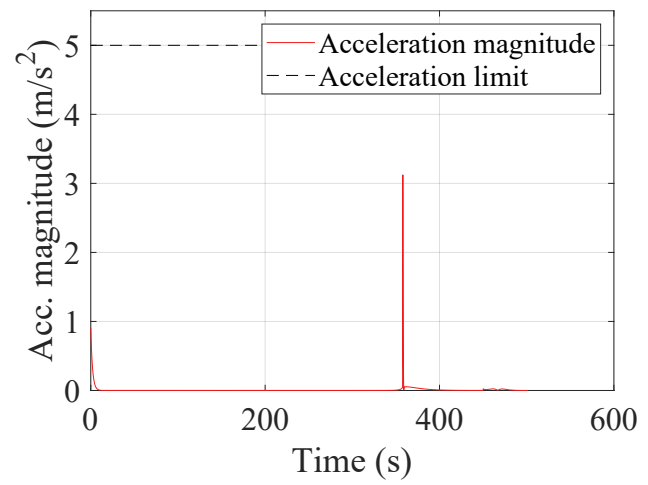

(d) Weight set 4.

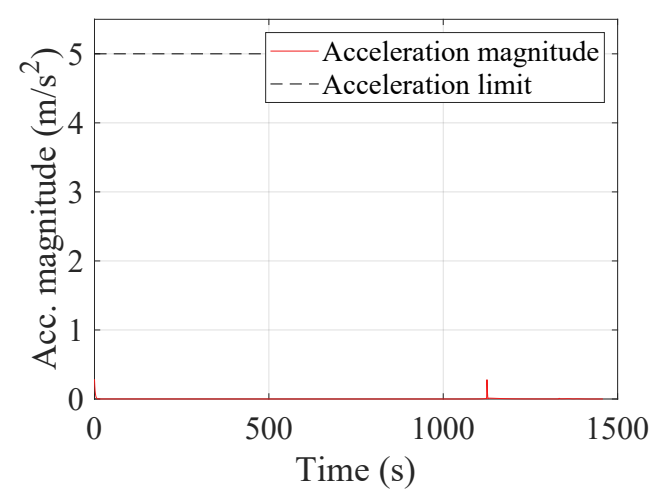

(e) Weight set 5 .

Figure 6.17: Initial condition set $1, u_{\max }=5.0 \mathrm{~m} / \mathrm{s}^{2}$, varying weigh sets. 


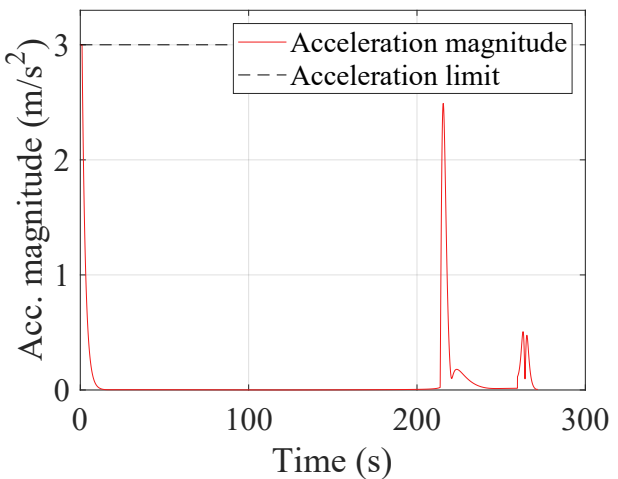

(a) Weight set 1 .

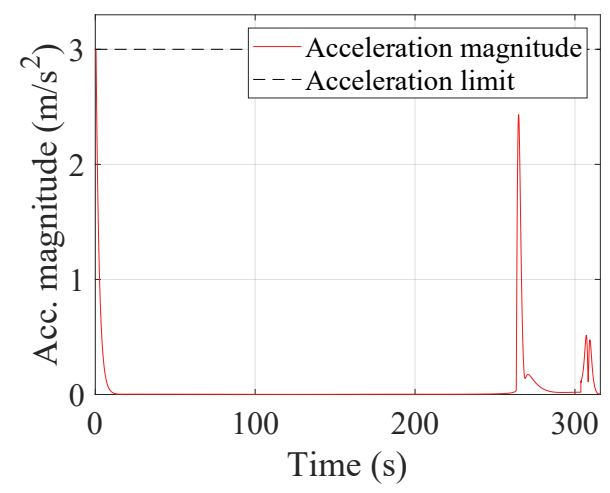

(c) Weight set 3 .

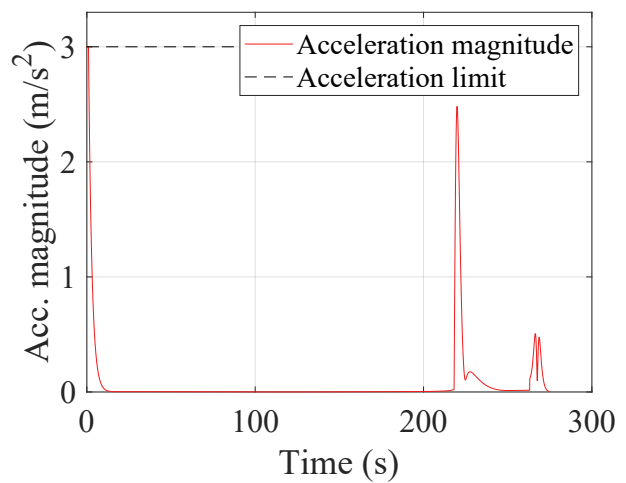

(b) Weight set 2 .

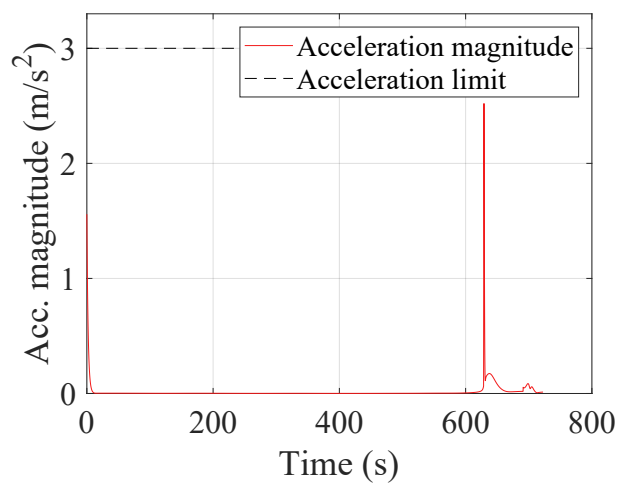

(d) Weight set 4.

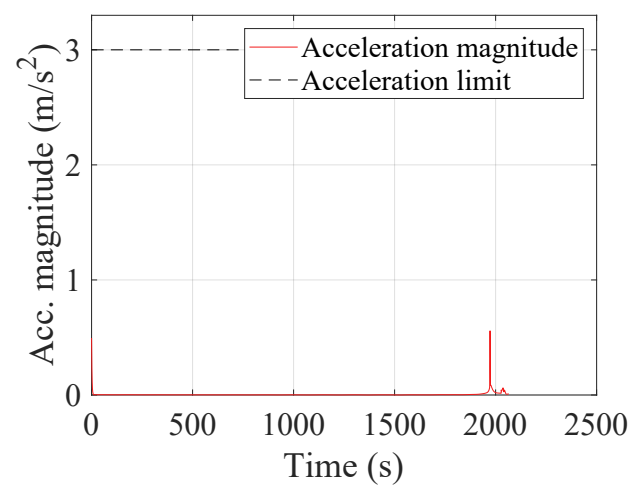

(e) Weight set 5 .

Figure 6.18: Initial condition set $2, u_{\max }=3.0 \mathrm{~m} / \mathrm{s}^{2}$, varying weigh sets. 
To illustrate this point, let us first observe several cases where the fuel optimization works very well. For example, Fig. 6.19 shows the actual versus estimated fuel usage for initial condition set 1 when $u_{\max }=5.0 \mathrm{~m} / \mathrm{s}^{2}$ as the weight set is varied. Note that the lower values of estimated fuel usage $\Delta \hat{\nu}_{P 1}$ correspond to cases where the fuel usage is penalized more in the cost function. Clearly, as this specific simulation case and $u_{\max }$ are held constant, the estimated fuel usage correlates very well to the actual fuel usage when weights are varied. Therefore, when the estimated fuel usage $\Delta \hat{\nu}_{P 1}\left(b, k_{c}, k_{a}\right)$ is penalized in the cost function, this results in reductions in the actual fuel usage $\Delta \nu_{P 1}$. Figure 6.20 shows similar lines, where each line represents one constant combination of simulation case and $u_{\max }$. The cases shown specifically in Fig. 6.20 are for initial condition sets 1 and 2 , and for $u_{\max }=\{1.0,2.0,3.0,4.0,5.0\} \mathrm{m} / \mathrm{s}^{2}$. While the individual lines are not very discernible, this nevertheless demonstrates that there exists a clear correlation for all cases at lower angular rates.

When similar plots are created for higher angular rates, however, a lesser correlation exists between $\Delta \hat{\nu}_{P 1}$ and $\Delta \nu_{P 1}$. For example, Fig. 6.21 shows the estimated and actual fuel usage for initial conditions set 6 , where there is a line for each of $u_{\max }=\{1.0,2.0,3.0,4.0,5.0\} \mathrm{m} / \mathrm{s}^{2}$. It is clear that for low values of $\Delta \hat{\nu}_{P 1}$ (corresponding to high penalties for fuel and very low penalties for time), several of these lines show a sharp increase in $\Delta \nu_{P 1}$ as $\Delta \hat{\nu}_{P 1}$ decreases. Therefore, although the optimizer successfully lowers the estimated fuel use, it does not correspond to a true drop in fuel. Because this effect is not observed for lesser target angular velocities, it is very likely that the rather rough estimation of required acceleration due to target angular velocity given by Eq. (5.81) is the cause of this sudden drop off in correlation. Refining this acceleration estimate would improve the optimization results for higher angular velocities.

It is still interesting to note that the parameter optimization works well for higher angular velocities when the weight disparity is not as large. Indeed, the reduced disparity between weights appears to make the algorithm perform more robustly to high angular velocities. It should be noted that the estimated and actual maneuver time remain quite correlated for all cases. For example, Fig. 6.22 shows the time estimation curves for the identical cases considered in Fig. 6.21. 
For Phase 1, Fig. 6.23 shows on a single graph the actual versus estimated fuel usage for each combination of simulation case and $u_{\max }$ as the weight set is varied. Likewise, Fig. 6.24 shows the actual versus estimated maneuver time for each combination of simulation case and $u_{\max }$. The lines are grouped by their color and marker according to simulation case, and the particular value of $u_{\max }$ is not distinguished in these plots. It is quite clear from these figures that $\Delta \hat{\nu}_{P 1}$ is less correlated with $\Delta \nu_{P 1}$ than $\hat{T}_{P 1}$ is with $T_{P 1}$. In Fig. 6.25, a similar graph is created for $\Delta \nu_{P 1}$ versus $\Delta \hat{\nu}_{P 1}$ where the last weight set is omitted. The correlation qualitatively appears substantially better.

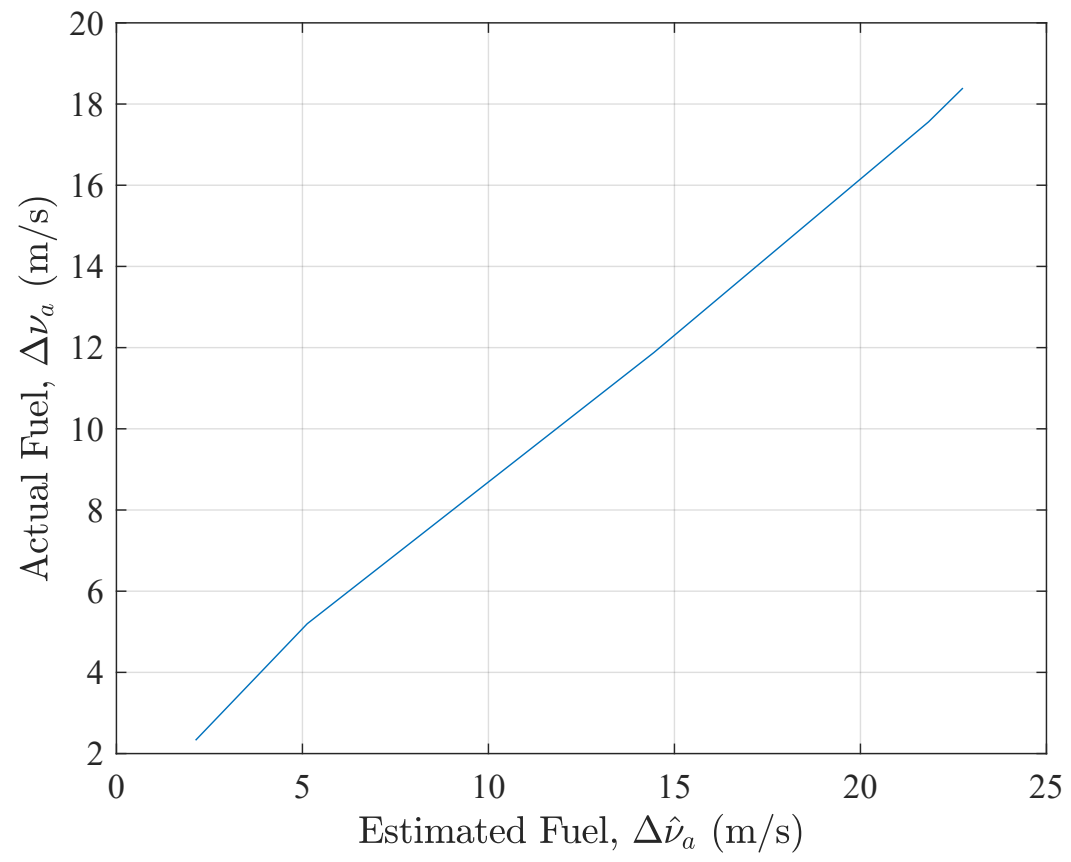

Figure 6.19: Actual versus estimated $\Delta \nu_{P 1}$ for initial condition set $1, u_{\max }=5.0$ $\mathrm{m} / \mathrm{s}^{2}$, across all weight sets. 


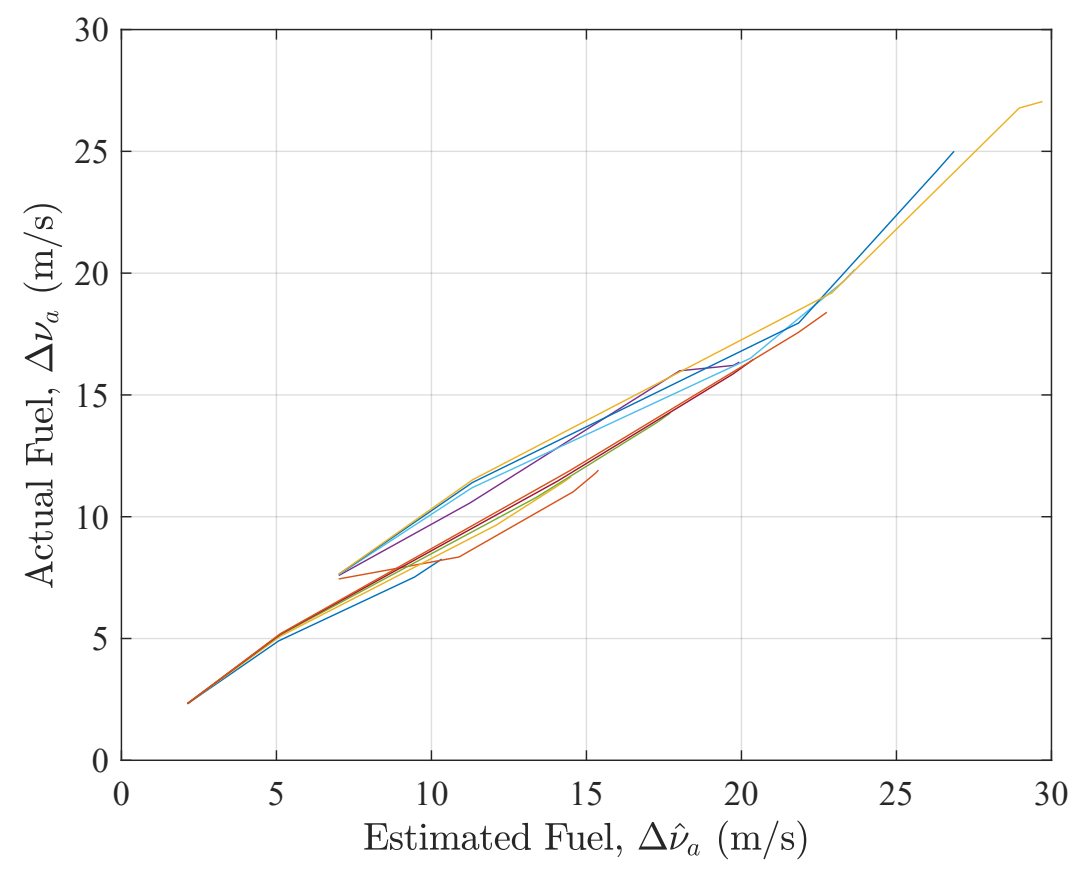

Figure 6.20: Actual versus estimated $\Delta \nu_{P 1}$ for initial condition set 1 and $2, u_{\max }=$ $\{1.0,2.0,3.0,4.0,5.0\} \mathrm{m} / \mathrm{s}^{2}$, across all weight sets.

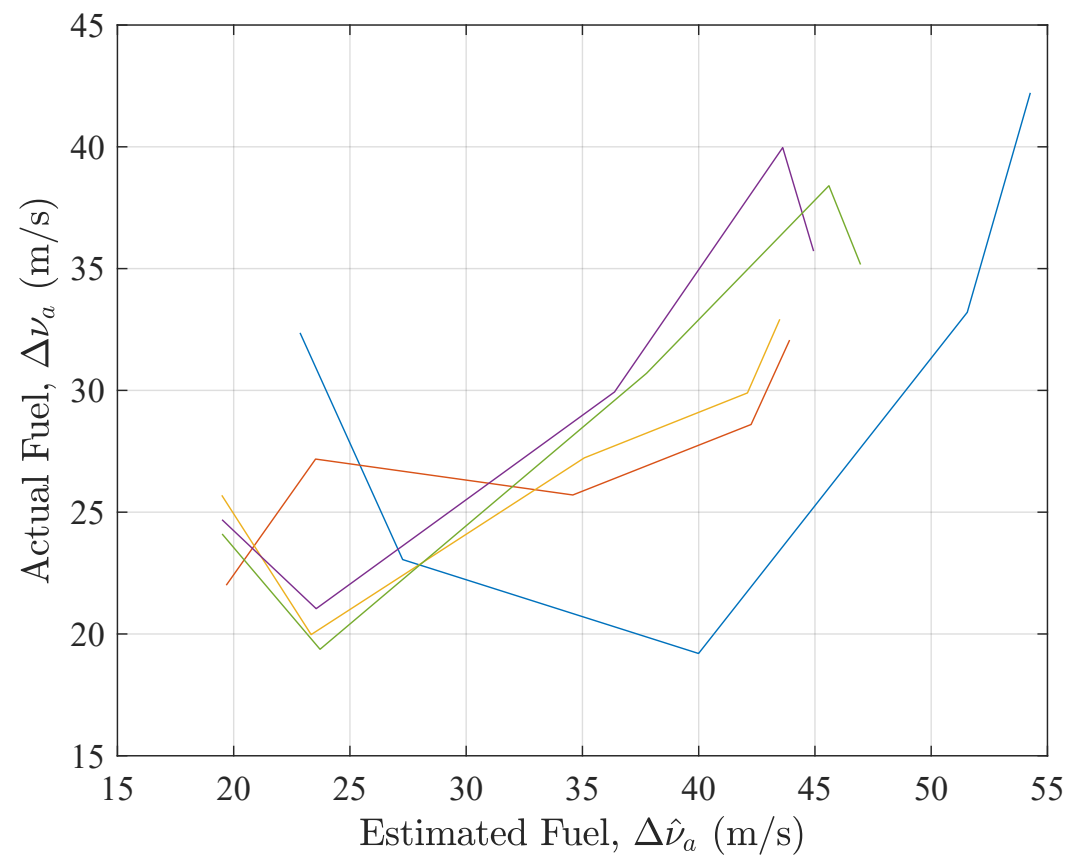

Figure 6.21: Actual versus estimated $\Delta \nu_{P 1}$ for initial condition set $6, u_{\max }=$ $\{1.0,2.0,3.0,4.0,5.0\} \mathrm{m} / \mathrm{s}^{2}$, across all weight sets. 


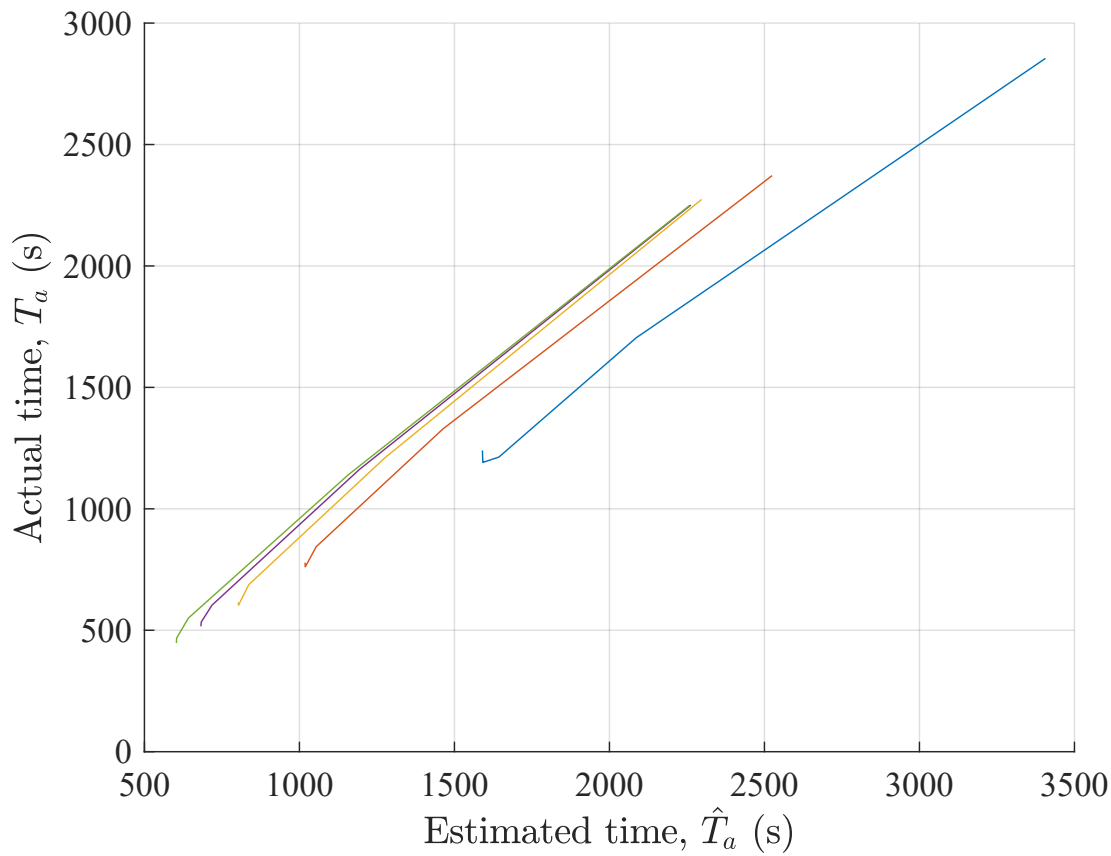

Figure 6.22: Actual versus estimated $T_{P 1}$ for initial condition set $6, u_{\max }=$ $\{1.0,2.0,3.0,4.0,5.0\} \mathrm{m} / \mathrm{s}^{2}$, across all weight sets.

\section{Observations of the Phase 2 performance variation}

The first observation to be noted is that the performance of the LVF is less variant to cost weighting when compared to the CLVF. This is again because Eq. (5.61) is quite a loose-bound. That is, because the feasible region of $v_{\max }$ is quite restricted by Eq. (5.61), variation of the weights makes little difference to the final $v_{\text {max }}$ selection, resulting in similar performance. This effect is again most apparent for higher target angular rates where Eq. (5.61) becomes most restrictive.

Consider, for example, the three simulation cases where the most significant performance variation is observed (namely, simulation cases 1, 2 and 3). In these particular cases, the target has relatively low rotational motion, which results in a far tighter bound on the LVF feasible parameter region because several of the terms in Eq. (5.61) go to 0 . Figures 6.26 and 6.27 show the actual variation in fuel and time performance versus the estimated variation. Again, each line represents holding the simulation case and $u_{\max }$ value constant while only the weight set is varied. Clearly, there are not the same fuel correlation issues which were observed for Phase 1. Rather, as the 


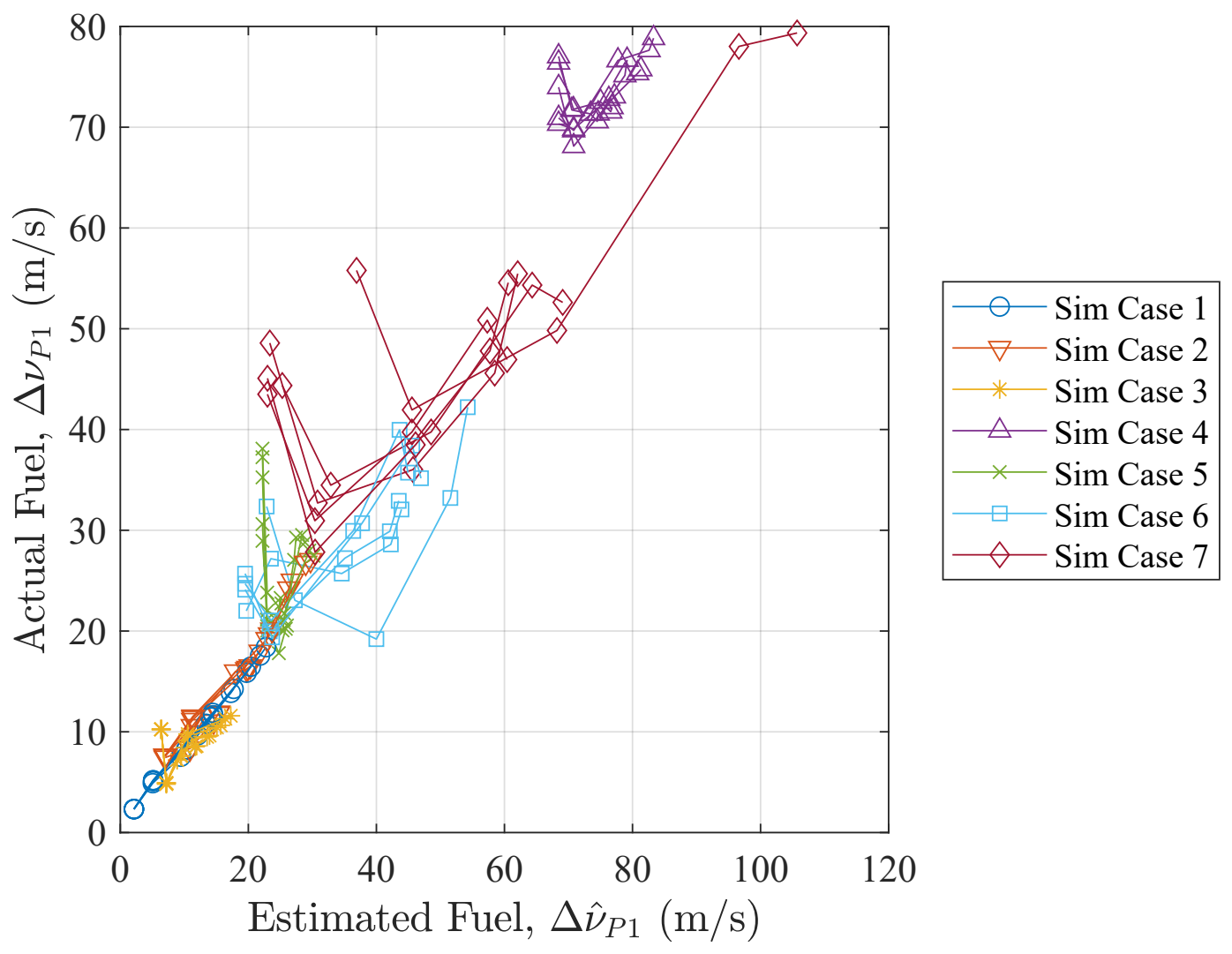

Figure 6.23: Actual versus estimated fuel usage for Phase 1 over 175 cases. 


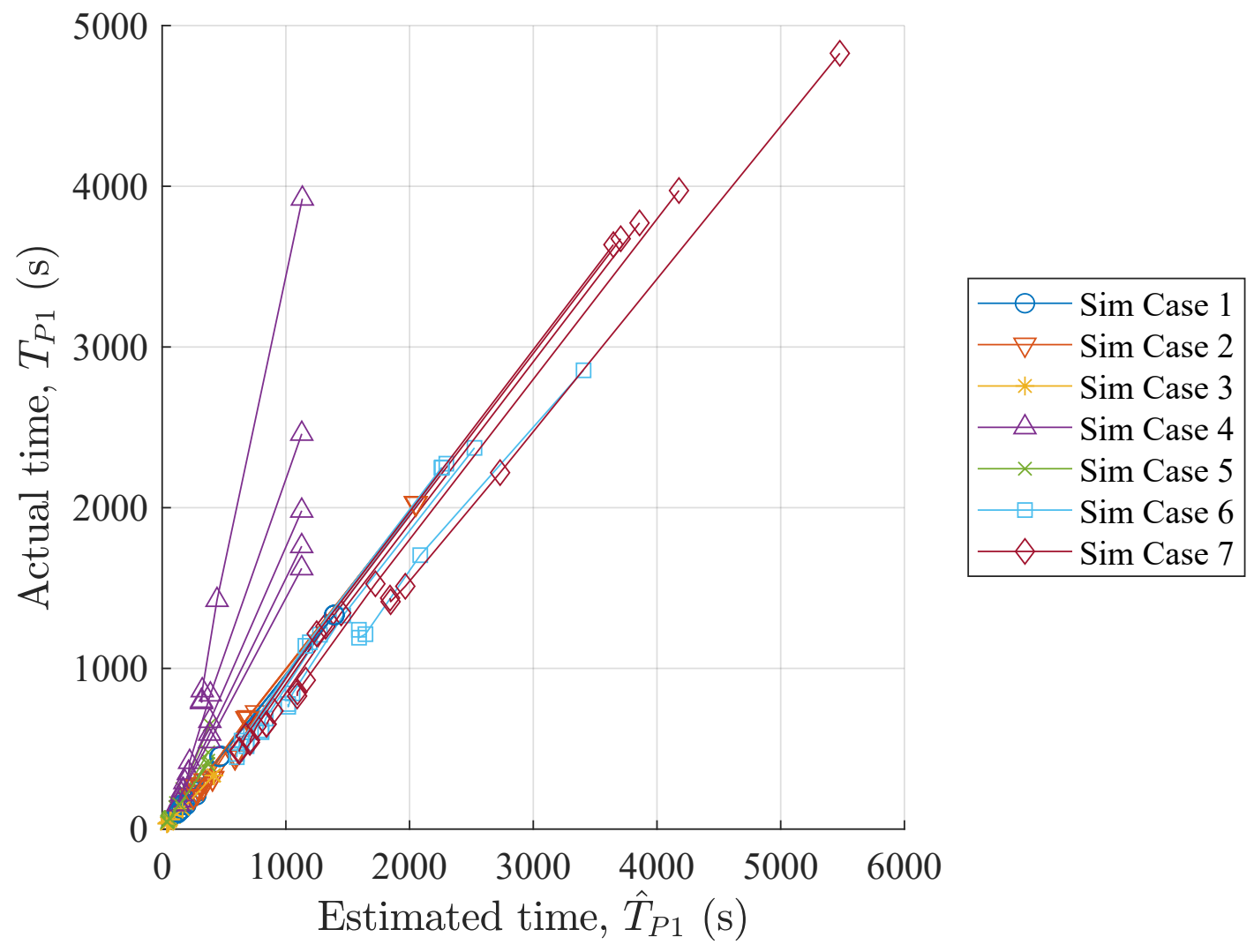

Figure 6.24: Actual versus estimated maneuver time for Phase 1 over 175 cases. 


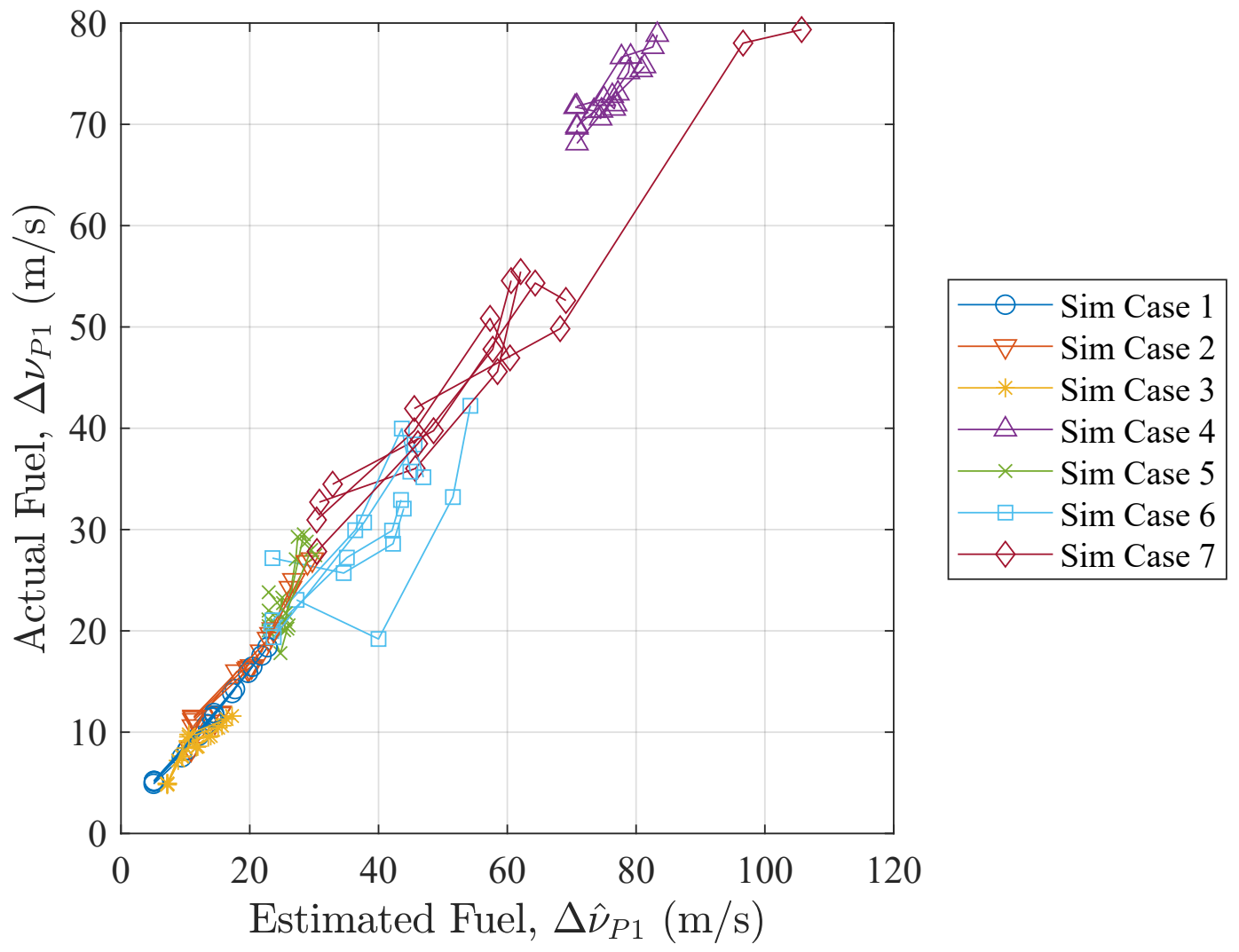

Figure 6.25: Actual versus estimated fuel usage for Phase 1, omitting weight set 5. 
angular velocity of the target increases, the variation of performance with weight gets "squished" until the performance is virtually invariant to the weights. It should be noted that the time estimates for Phase 2 do not simply correlate to the actual time for each case. Rather, as seen from Fig. 6.27, the time estimate given by Eq. (5.89) correspond to almost an exact value of the true convergence time. This is as expected, as the time to complete Phase 2 has no variation to the target angular velocity or the chasers initial conditions. Therefore, the Phase 2 maneuver time is the easiest metric to approximate.

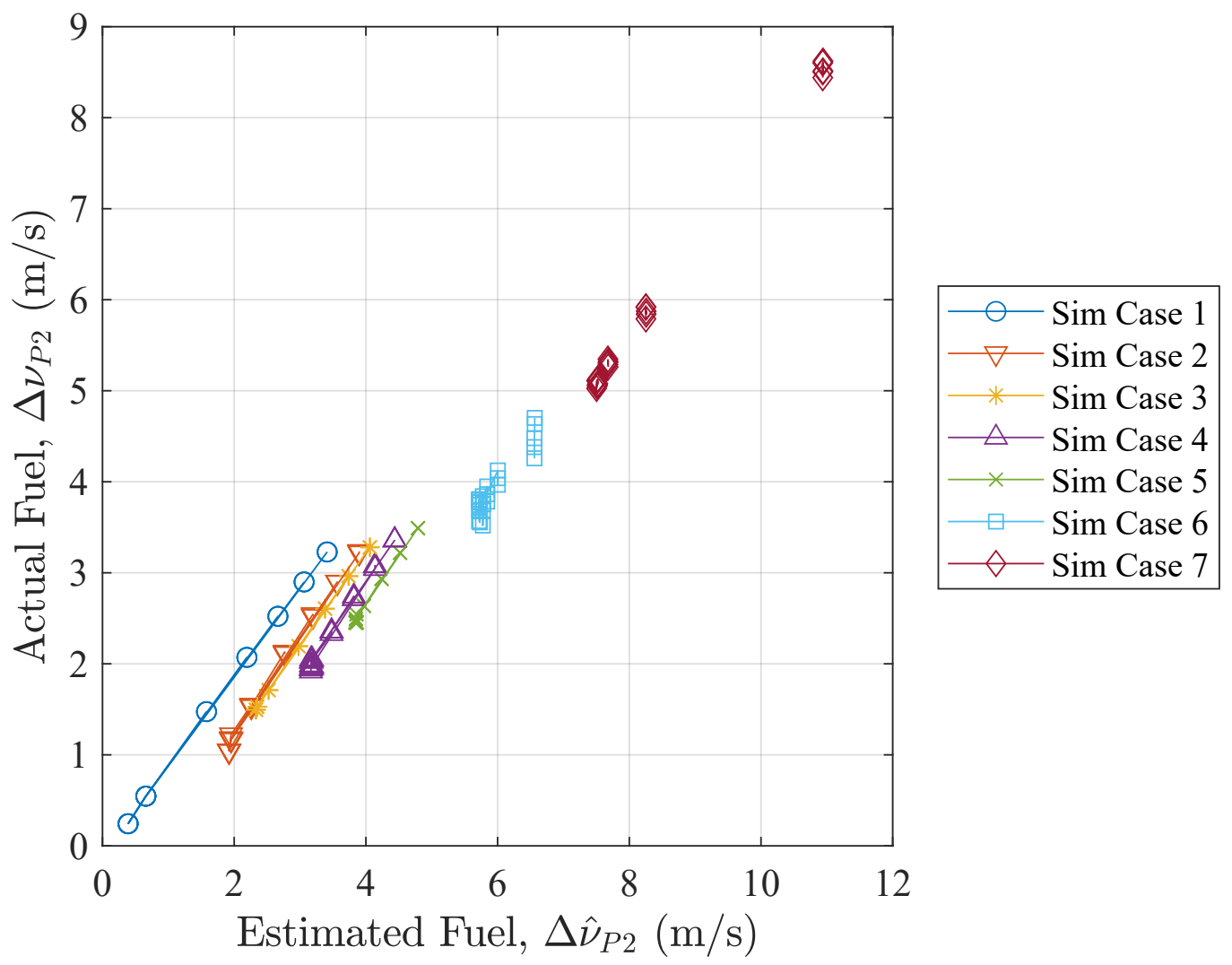

Figure 6.26: Actual versus estimated fuel usage for Phase 1 over 175 cases. 


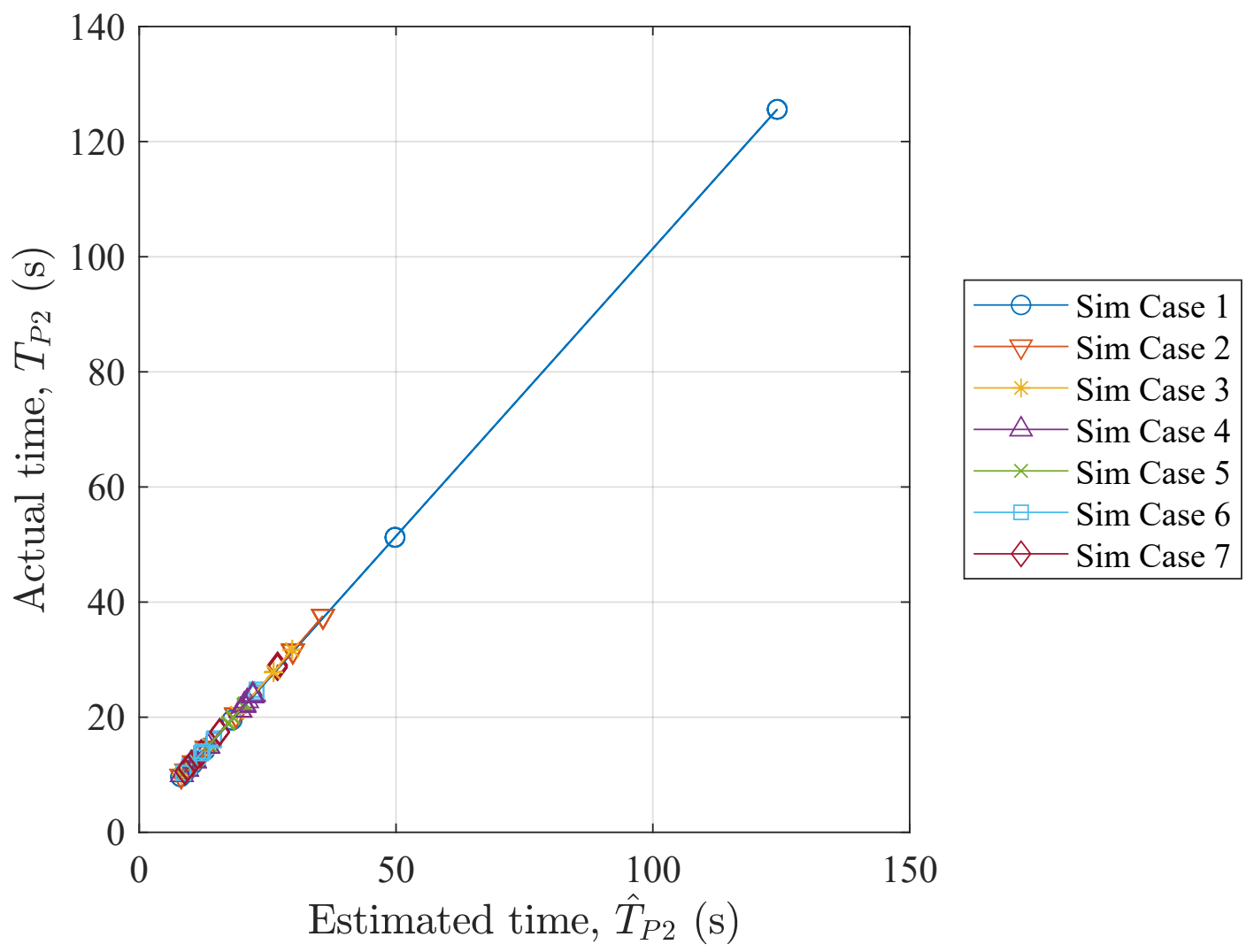

Figure 6.27: Actual versus estimated maneuver time for Phase 1 over 175 cases. 
Table 6.5: Actual $\Delta \nu_{P 1}(\mathrm{~m} / \mathrm{s})$ for weight case 1 .

\begin{tabular}{|c|c|c|c|c|c|c|c|}
\hline & \multicolumn{7}{|c|}{ Simulation Case } \\
\hline & 1 & 2 & 3 & 4 & 5 & 6 & 7 \\
\hline 1.0 & 8.262 & 11.913 & 7.628 & 71.352 & 22.737 & 42.215 & 79.354 \\
\hline$\stackrel{\infty}{>} 2.0$ & 11.641 & 16.346 & 8.599 & 71.983 & 20.547 & 32.065 & 52.615 \\
\hline$\Xi 3.0$ & 14.256 & 20.148 & 9.632 & 76.683 & 29.275 & 32.919 & 55.462 \\
\hline 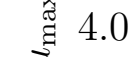 & 16.462 & 25.004 & 10.640 & 75.740 & 28.803 & 35.718 & 46.942 \\
\hline$\curvearrowright 5.0$ & 18.393 & 27.047 & 11.591 & 78.837 & 27.465 & 35.169 & 54.571 \\
\hline
\end{tabular}

Table 6.6: Actual $\Delta \nu_{P 1}(\mathrm{~m} / \mathrm{s})$ for weight case 2 .

\begin{tabular}{|c|c|c|c|c|c|c|c|}
\hline & \multicolumn{7}{|c|}{ Simulation Case } \\
\hline & 1 & 2 & 3 & 4 & 5 & 6 & 7 \\
\hline 1.0 & 8.189 & 11.801 & 7.584 & 70.580 & 23.284 & 33.205 & 78.018 \\
\hline$\stackrel{\infty}{>} 2.0$ & 11.429 & 16.210 & 8.533 & 71.548 & 20.220 & 28.600 & 54.330 \\
\hline घ 3.0 & 13.866 & 19.673 & 9.503 & 75.144 & 27.064 & 29.896 & 45.594 \\
\hline gु 4.0 & 15.860 & 24.226 & 10.436 & 75.349 & 29.531 & 39.968 & 50.846 \\
\hline న 5.0 & 17.560 & 26.776 & 11.299 & 77.642 & 27.986 & 38.402 & 47.798 \\
\hline
\end{tabular}

Table 6.7: Actual $\Delta \nu_{P 1}(\mathrm{~m} / \mathrm{s})$ for weight case 3 .

\begin{tabular}{|c|c|c|c|c|c|c|c|}
\hline & \multicolumn{7}{|c|}{ Simulation Case } \\
\hline & 1 & 2 & 3 & 4 & 5 & 6 & 7 \\
\hline N 1.0 & 7.523 & 11.028 & 7.203 & 71.319 & 20.552 & 19.199 & 49.828 \\
\hline${ }_{\infty}^{\infty} 2.0$ & 9.645 & 15.991 & 8.423 & 72.511 & 17.800 & 25.706 & 39.754 \\
\hline$\exists 3.0$ & 10.797 & 16.503 & 9.144 & 72.799 & 20.066 & 27.229 & 36.024 \\
\hline हี 4.0 & 11.465 & 17.953 & 9.542 & 73.050 & 21.112 & 29.935 & 39.746 \\
\hline$\curvearrowright 5.0$ & 11.878 & 19.200 & 9.775 & 76.630 & 21.743 & 30.702 & 38.481 \\
\hline
\end{tabular}

Table 6.8: Actual $\Delta \nu_{P 1}(\mathrm{~m} / \mathrm{s})$ for weight case 4 .

\begin{tabular}{|c|c|c|c|c|c|c|c|}
\hline & \multicolumn{7}{|c|}{ "Simulation Case } \\
\hline & 1 & 2 & 3 & 4 & 5 & 6 & 7 \\
\hline 1.0 & 4.901 & 8.347 & 4.809 & 71.713 & 21.130 & 23.054 & 41.946 \\
\hline$\stackrel{\infty}{=} 2.0$ & 5.086 & 10.571 & 4.890 & 71.779 & 20.467 & 27.181 & 34.475 \\
\hline घ 3.0 & 5.150 & 11.187 & 4.908 & 69.824 & 20.047 & 19.974 & 32.707 \\
\hline 苛 4.0 & 5.178 & 11.406 & 4.916 & 68.120 & 22.002 & 21.039 & 30.949 \\
\hline 5.0 & 5.199 & 11.521 & 4.921 & 69.681 & 23.812 & 19.371 & 27.832 \\
\hline
\end{tabular}


Table 6.9: Actual $\Delta \nu_{P 1}(\mathrm{~m} / \mathrm{s})$ for weight case 5 .

\begin{tabular}{|c|c|c|c|c|c|c|c|}
\hline & \multicolumn{7}{|c|}{ Simulation Case } \\
\hline & 1 & 2 & 3 & 4 & 5 & 6 & 7 \\
\hline 1.0 & 2.318 & 7.445 & 10.248 & 77.032 & 35.249 & 32.362 & 55.789 \\
\hline${ }_{-\infty}^{\infty} 2.0$ & 2.325 & 7.586 & 10.245 & 76.404 & 37.249 & 22.002 & 44.362 \\
\hline घ 3.0 & 2.327 & 7.621 & 10.245 & 70.330 & 30.613 & 25.692 & 48.582 \\
\hline छु 4.0 & 2.328 & 7.635 & 10.245 & 73.972 & 28.946 & 24.689 & 43.499 \\
\hline$\therefore 5.0$ & 2.328 & 7.644 & 10.243 & 70.916 & 38.098 & 24.105 & 45.082 \\
\hline
\end{tabular}

Table 6.10: Actual $T_{P 1}(\mathrm{~s})$ for weight case 1 .

\begin{tabular}{|c|c|c|c|c|c|c|c|}
\hline & \multicolumn{7}{|c|}{ Simulation Case } \\
\hline & 1 & 2 & 3 & 4 & 5 & 6 & 7 \\
\hline N 1.0 & 210.650 & 447.400 & 76.350 & 798.350 & 135.050 & 1240.300 & 1437.500 \\
\hline$\stackrel{\infty}{>} 2.0$ & 149.600 & 315.300 & 52.100 & 347.600 & 74.100 & 778.400 & 828.200 \\
\hline$\exists 3.0$ & 123.250 & 259.700 & 42.550 & 240.500 & 62.750 & 614.700 & 653.700 \\
\hline छี 4.0 & 107.700 & 227.600 & 37.150 & 176.900 & 49.550 & 516.100 & 539.500 \\
\hline ₹ 5.0 & 97.100 & 201.600 & 33.600 & 154.000 & 42.600 & 447.700 & 482.700 \\
\hline
\end{tabular}

Table 6.11: Actual $T_{P 1}(\mathrm{~s})$ for weight case 2 .

\begin{tabular}{|c|c|c|c|c|c|c|c|}
\hline & \multicolumn{7}{|c|}{ Simulation Case } \\
\hline & 1 & 2 & 3 & 4 & 5 & 6 & 7 \\
\hline 1.0 & 212.350 & 448.500 & 76.850 & 789.650 & 137.700 & 1191.050 & 1415.050 \\
\hline$\stackrel{\infty}{\infty} 2.0$ & 152.000 & 318.750 & 52.950 & 350.100 & 74.450 & 761.400 & 853.900 \\
\hline$\nsubseteq 3.0$ & 126.200 & 263.100 & 43.600 & 243.250 & 63.600 & 603.400 & 652.750 \\
\hline$\stackrel{\Xi}{\approx}$ & 111.100 & 232.150 & 38.400 & 182.750 & 51.650 & 533.350 & 553.400 \\
\hline × 5.0 & 101.000 & 207.650 & 35.050 & 157.550 & 44.700 & 467.900 & 491.600 \\
\hline
\end{tabular}

Table 6.12: Actual $T_{P 1}(\mathrm{~s})$ for weight case 3 .

\begin{tabular}{|c|c|c|c|c|c|c|c|}
\hline & \multicolumn{7}{|c|}{ "Simulation Case } \\
\hline & 1 & 2 & 3 & 4 & 5 & 6 & 7 \\
\hline 1.0 & 230.750 & 468.300 & 82.350 & 863.300 & 161.900 & 1213.100 & 1511.000 \\
\hline${ }_{-1}^{\infty} 2.0$ & 179.500 & 357.750 & 64.500 & 417.800 & 74.750 & 844.100 & 926.650 \\
\hline$\exists 3.0$ & 161.800 & 303.750 & 61.200 & 291.850 & 70.350 & 687.750 & 735.500 \\
\hline छ్g 4.0 & 153.900 & 278.650 & 60.450 & 241.400 & 67.400 & 603.250 & 638.200 \\
\hline$\approx 5.0$ & 149.850 & 264.500 & 60.150 & 216.100 & 66.500 & 550.350 & 579.300 \\
\hline
\end{tabular}


Table 6.13: Actual $T_{P 1}(\mathrm{~s})$ for weight case 4 .

\begin{tabular}{|c|c|c|c|c|c|c|c|}
\hline & \multicolumn{7}{|c|}{ Simulation Case } \\
\hline & 1 & 2 & 3 & 4 & 5 & 6 & 7 \\
\hline 1.0 & 456.500 & 726.900 & 127.400 & 1425.700 & 249.000 & 1704.500 & 2218.050 \\
\hline 2.0 & 451.450 & 696.550 & 126.900 & 837.350 & 169.700 & 1329.300 & 1526.300 \\
\hline 3.0 & 450.550 & 691.100 & 126.850 & 672.700 & 153.250 & 1209.650 & 1345.400 \\
\hline$\stackrel{\varkappa}{\because} 4.0$ & 450.250 & 689.200 & 126.850 & 593.200 & 153.700 & 1162.350 & 1262.350 \\
\hline 5.0 & 450.100 & 688.350 & 126.850 & 546.700 & 153.800 & 1140.500 & 1215.950 \\
\hline
\end{tabular}

Table 6.14: Actual $T_{P 1}(\mathrm{~s})$ for weight case 5 .

\begin{tabular}{|c|c|c|c|c|c|c|c|}
\hline & \multicolumn{7}{|c|}{ Simulation Case } \\
\hline & 1 & 2 & 3 & 4 & 5 & 6 & 7 \\
\hline 1.0 & 1332.900 & 2029.150 & 334.100 & 3922.750 & 650.650 & 2854.800 & 4827.50 \\
\hline 2.0 & 1332.450 & 2027.900 & 334.000 & 2459.600 & 477. & 2372.050 & 3973. \\
\hline 3.0 & 1332.300 & 2027.650 & 334.000 & 1981.200 & 425.550 & 2273.800 & 3771.850 \\
\hline 4.0 & 1332.250 & 2027.500 & 334.000 & 1760.500 & 404.450 & 2250.800 & 3673.450 \\
\hline 5.0 & 1332.200 & 2027.450 & 333.950 & 1622.400 & 403.800 & 2247.700 & 3636.750 \\
\hline
\end{tabular}

Table 6.15: Actual $\Delta \nu_{P 2}(\mathrm{~m} / \mathrm{s})$ for weight case 1 .

\begin{tabular}{|c|c|c|c|c|c|c|c|}
\hline & \multicolumn{7}{|c|}{ Simulation Case } \\
\hline & 1 & 2 & 3 & 4 & 5 & 6 & 7 \\
\hline & 1.474 & 1.532 & 1.712 & 1.956 & 2.542 & 4.258 & 8.440 \\
\hline 2.0 & 2.071 & 2.114 & 2.194 & 2.358 & 2.636 & 3.685 & 5.789 \\
\hline 3.0 & 2.522 & 2.525 & 2.606 & 2.716 & 2.937 & 3.676 & 5.347 \\
\hline 4.0 & 2.900 & 2.898 & 2.963 & 3.074 & 3.219 & 3.782 & 5.114 \\
\hline 5.0 & 3.229 & 3.236 & 3.279 & 3.361 & 3.488 & 4.039 & 5.050 \\
\hline
\end{tabular}

Table 6.16: Actual $\Delta \nu_{P 2}(\mathrm{~m} / \mathrm{s})$ for weight case 2 .

\begin{tabular}{|c|c|c|c|c|c|c|c|}
\hline & \multicolumn{7}{|c|}{ Simulation Case } \\
\hline & 1 & 2 & 3 & 4 & 5 & 6 & 7 \\
\hline & 1.474 & 1.533 & 1.712 & 1.973 & 2.546 & 4.381 & 8.601 \\
\hline 22.0 & 2.071 & 2.117 & 2.194 & 2.359 & 2.637 & 3.841 & 5.920 \\
\hline$\exists 3.0$ & 2.522 & 2.526 & 2.606 & 2.716 & 2.932 & 3.774 & 5.287 \\
\hline ध 4.0 & 2.900 & 2.896 & 2.963 & 3.078 & 3.221 & 3.863 & 5.069 \\
\hline 5.0 & 3.229 & 3.233 & 3.280 & 3.360 & 3.491 & 3.967 & 5.062 \\
\hline
\end{tabular}


Table 6.17: Actual $\Delta \nu_{P 2}(\mathrm{~m} / \mathrm{s})$ for weight case 3 .

\begin{tabular}{|c|c|c|c|c|c|c|c|}
\hline & \multicolumn{7}{|c|}{ Simulation Case } \\
\hline & 1 & 2 & 3 & 4 & 5 & 6 & 7 \\
\hline 1.0 & 1.475 & 1.548 & 1.711 & 2.047 & 2.446 & 4.479 & 8.503 \\
\hline 2.0 & 2.071 & 2.130 & 2.194 & 2.331 & 2.637 & 3.519 & 5.921 \\
\hline 3.0 & 2.522 & 2.543 & 2.606 & 2.744 & 2.931 & 3.554 & 5.307 \\
\hline 4.0 & 2.899 & 2.899 & 2.964 & 3.045 & 3.218 & 3.948 & 5.071 \\
\hline 5.0 & 3.228 & 3.220 & 3.281 & 3.357 & 3.492 & 4.124 & 5.120 \\
\hline
\end{tabular}

Table 6.18: Actual $\Delta \nu_{P 2}(\mathrm{~m} / \mathrm{s})$ for weight case 4 .

\begin{tabular}{|c|c|c|c|c|c|c|c|}
\hline & \multicolumn{7}{|c|}{ Simulation Case } \\
\hline & 1 & 2 & 3 & 4 & 5 & 6 & 7 \\
\hline 1.0 & 0.545 & 1.222 & 1.527 & 2.051 & 2.460 & 4.634 & 8.513 \\
\hline 2.0 & 0.546 & 1.179 & 1.529 & 2.037 & 2.469 & 3.752 & 5.841 \\
\hline 3.0 & 0.546 & 1.171 & 1.528 & 2.027 & 2.491 & 3.808 & 5.322 \\
\hline 4.0 & 0.546 & 1.168 & 1.528 & 1.971 & 2.492 & 3.746 & 5.079 \\
\hline 5.0 & 0.546 & 1.167 & 1.528 & 2.049 & 2.495 & 3.689 & 5.025 \\
\hline
\end{tabular}

Table 6.19: Actual $\Delta \nu_{P 2}(\mathrm{~m} / \mathrm{s})$ for weight case 5 .

\begin{tabular}{|c|c|c|c|c|c|c|c|}
\hline & \multicolumn{7}{|c|}{ Simulation Case } \\
\hline & 1 & 2 & 3 & 4 & 5 & 6 & 7 \\
\hline 1.0 & 0.243 & 1.042 & 1.496 & 1.919 & 2.452 & 4.702 & 8.621 \\
\hline $\mathbb{\infty}_{-\infty}^{\infty} 2.0$ & 0.243 & 1.042 & 1.496 & 1.943 & 2.453 & 3.756 & 5.873 \\
\hline$\exists \quad 3.0$ & 0.243 & 1.042 & 1.496 & 1.957 & 2.457 & 3.568 & 5.261 \\
\hline 关 4.0 & 0.243 & 1.042 & 1.496 & 1.988 & 2.452 & 3.802 & 5.132 \\
\hline$\approx 5.0$ & 0.243 & 1.042 & 1.496 & 2.025 & 2.452 & 3.781 & 5.109 \\
\hline
\end{tabular}

Table 6.20: Actual $T_{P 2}(\mathrm{~s})$ for weight case 1 .

\begin{tabular}{|c|c|c|c|c|c|c|c|}
\hline & \multicolumn{7}{|c|}{ Simulation Case } \\
\hline & 1 & 2 & 3 & 4 & 5 & 6 & 7 \\
\hline 1.0 & 19.500 & 20.350 & 20.750 & 21.150 & 22.150 & 24.200 & 28.600 \\
\hline 赵 2.0 & 14.200 & 14.600 & 14.750 & 14.900 & 15.200 & 16.000 & 17.350 \\
\hline E 3.0 & 11.850 & 12.050 & 12.250 & 12.350 & 12.550 & 13.000 & 13.750 \\
\hline 䴔 4.0 & 10.550 & 10.700 & 10.800 & 10.900 & 11.100 & 11.450 & 11.750 \\
\hline 5.0 & 9.650 & 9.800 & 9.900 & 9.950 & 10.100 & 10.300 & 10.600 \\
\hline
\end{tabular}


Table 6.21: Actual $T_{P 2}(\mathrm{~s})$ for weight case 2 .

\begin{tabular}{|c|c|c|c|c|c|c|c|}
\hline & \multicolumn{7}{|c|}{ Simulation Case } \\
\hline & 1 & 2 & 3 & 4 & 5 & 6 & 7 \\
\hline 1.0 & 19.500 & 20.350 & 20.750 & 21.150 & 22.150 & 24.150 & 28.950 \\
\hline 2.0 & 14.200 & 14.650 & 14.750 & 14.900 & 15.200 & 16.200 & 17.400 \\
\hline 3.0 & 11.850 & 12.050 & 12.250 & 12.350 & 12.550 & 13.050 & 13.650 \\
\hline 疱 4.0 & 10.550 & 10.700 & 10.800 & 10.950 & 11.100 & 11.500 & 11.850 \\
\hline 5.0 & 9.650 & 9.800 & 9.900 & 9.950 & 10.100 & 10.350 & 10.550 \\
\hline
\end{tabular}

Table 6.22: Actual $T_{P 2}(\mathrm{~s})$ for weight case 3 .

\begin{tabular}{|c|c|c|c|c|c|c|c|}
\hline & \multicolumn{7}{|c|}{ Simulation Case } \\
\hline & 1 & 2 & 3 & 4 & 5 & 6 & 7 \\
\hline 1.0 & 19.500 & 20.500 & 20.700 & 21.200 & 21.800 & 24.350 & 28.650 \\
\hline 2.0 & 14.150 & 14.700 & 14.750 & 14.950 & 15.200 & 15.950 & 17.300 \\
\hline 3.0 & 11.850 & 12.150 & 12.250 & 12.450 & 12.550 & 13.050 & 13.700 \\
\hline 4.0 & 10.550 & 10.700 & 10.850 & 10.900 & 11.050 & 11.450 & 11.900 \\
\hline 5.0 & 9.650 & 9.800 & 9.900 & 10.000 & 10.050 & 10.350 & 10.550 \\
\hline
\end{tabular}

Table 6.23: Actual $T_{P 2}(\mathrm{~s})$ for weight case 4 .

\begin{tabular}{|c|c|c|c|c|c|c|c|}
\hline & \multicolumn{7}{|c|}{ Simulation Case } \\
\hline & 1 & 2 & 3 & 4 & 5 & 6 & 7 \\
\hline 1.0 & 51.300 & 31.600 & 27.950 & 22.800 & 22.300 & 24.750 & 28.900 \\
\hline 2.0 & 51.250 & 31.500 & 27.850 & 22.150 & 19.100 & 16.350 & 17.500 \\
\hline 3.0 & 51.250 & 31.500 & 27.800 & 22.000 & 18.850 & 13.900 & 13.850 \\
\hline 晃 4.0 & 51.250 & 31.500 & 27.800 & 22.000 & 18.850 & 13.750 & 11.950 \\
\hline 5.0 & 51.200 & 31.500 & 27.800 & 22.200 & 18.850 & 13.700 & 11.100 \\
\hline
\end{tabular}

Table 6.24: Actual $T_{P 2}(\mathrm{~s})$ for weight case 5 .

\begin{tabular}{|c|c|c|c|c|c|c|c|}
\hline & \multicolumn{7}{|c|}{ Simulation Case } \\
\hline & 1 & 2 & 3 & 4 & 5 & 6 & 7 \\
\hline 1.0 & 125.650 & 37.600 & 31.800 & 23.900 & 22.400 & 24.800 & 29.100 \\
\hline 2.0 & 125.600 & 37.550 & 31.700 & 23.800 & 19.950 & 16.250 & 17.550 \\
\hline 3.0 & 125.600 & 37.550 & 31.700 & 23.900 & 20.000 & 14.100 & 13.850 \\
\hline 4.0 & 125.600 & 37.550 & 31.700 & 23.850 & 19.950 & 14.050 & 12.000 \\
\hline 5.0 & 125.600 & 37.550 & 31.700 & 23.650 & 19.950 & 14.050 & 11.250 \\
\hline
\end{tabular}




\section{Chapter 7}

\section{Experimental Validation}

In this Chapter, the combined CLVF and LVF docking maneuver is tested in a realtime hardware environment. Note that, due to improvements which must be made to the optimizer performance, particularly in the fuel estimation of the CLVF (Phase 1), only the primary results of this thesis will be tested in experiment. Specifically, this Chapter aims to validate the ability of CLVFs and LVFs to meet the acceleration and path constraints while using real hardware with practical imperfections such as noise-corrupted measurements, thruster time-delays, imperfect thrust resolution, and perturbations such as air resistance. The parameter optimization technique developed in this thesis will still be used to select field parameters which meet acceleration constraints, but no variation in the weights will be tested.

The remainder of this chapter is organized as follows: Section 7.1 introduces the Spacecraft Proximity Operations Testbed (SPOT) facility located at Carleton University. Then, the various test cases are introduced in Sec. 7.2. Finally, the experimental data are analyzed in Sec. 7.3.

\subsection{Spacecraft Proximity Operations Testbed (SPOT) Facility}

The SPOT facility at Carleton University is a three degree-of-freedom testbed designed for experimental validation of spacecraft proximity algorithms. The facility consists are a large granite table (3.51 $\mathrm{m}$ in length by $2.42 \mathrm{~m}$ in width) and two robotic platforms, creatively named "RED" and "BLACK", shown in Fig 7.1. 


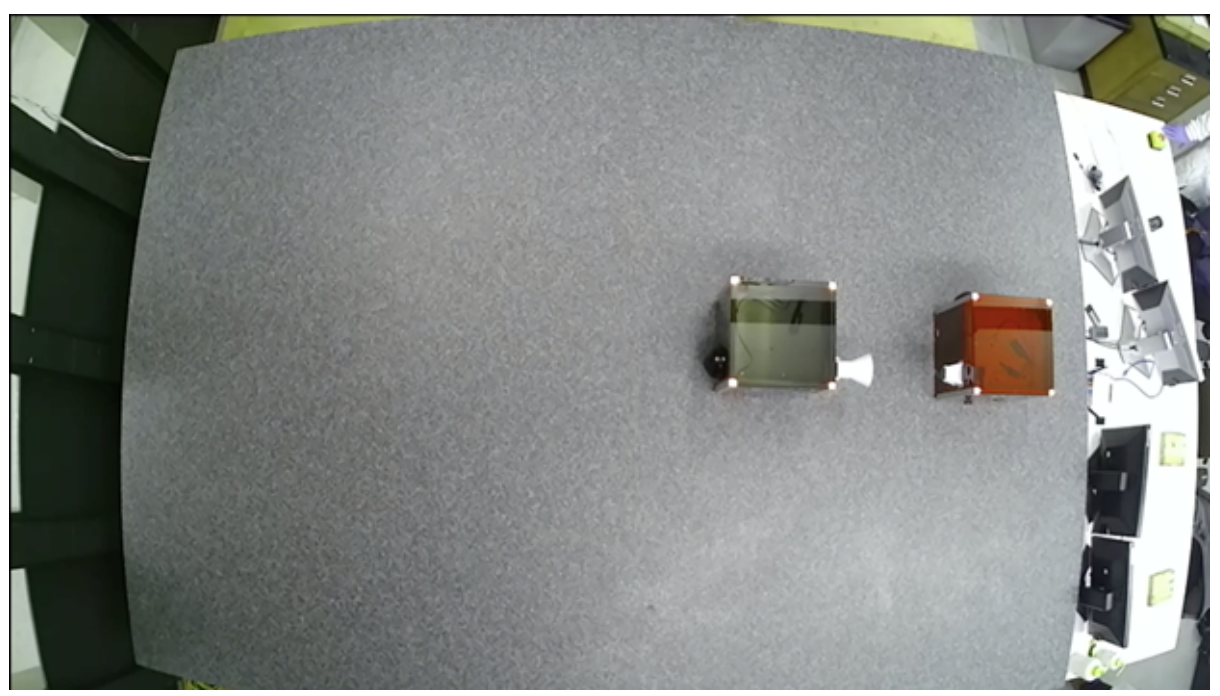

Figure 7.1: Top view of granite table and robotic platforms in SPOT facility.

The "inertial frame" of the granite table has an origin in the bottom left corner, which can be seen in the fish-eyed photo of Fig. 7.1. The inertial x-vector $\hat{\boldsymbol{X}}$ is defined along the long axis of the table, and the inertial y-vector $\hat{\boldsymbol{Y}}$ is along the short axis. Likewise, the positive x-vector of the RED body-fixed frame $\hat{\boldsymbol{x}}_{R}$ is defined by the direction where the mating attachment for RED is mounted. The body-fixed z-vector $\hat{\boldsymbol{z}}_{R}$ is upwards, and the body-fixed y-vector $\hat{\boldsymbol{y}}_{R}$ completes the right-handed triad. The BLACK body-fixed frame is defined similarly, except the mating attachment defines the body-fixed y-vector $\hat{\boldsymbol{y}}_{B}$. The attitude for each platform is defined by the angle between the inertial x-vector and the body-fixed x-vector, denoted $\kappa_{R}$ and $\kappa_{B}$ for RED and BLACK respectively. These reference frame and angle definitions are visualized in Fig. 7.2.

The robotic platforms both have on-board compressed air tanks which serve two purposes. Firstly, the compressed air is used as propellant through solenoid valves during experiment. Secondly, a small flow of air is injected underneath the platforms such that the friction between the platforms and the surface of the table is close to 0 , which emulates the friction-free environment of space. The RED platform has a nominal mass and moment of inertia of $16.9478 \mathrm{~kg}$ and $0.2709 \mathrm{~kg} \cdot \mathrm{m}^{2}$ respectively. Likewise, BLACK has a nominal mass and moment of inertia of $12.3341 \mathrm{~kg}$ and $0.1880 \mathrm{~kg} \cdot \mathrm{m}^{2}$ respectively. The maximum thrust capacity of each platform is approximately $0.4 \mathrm{~N}$. 


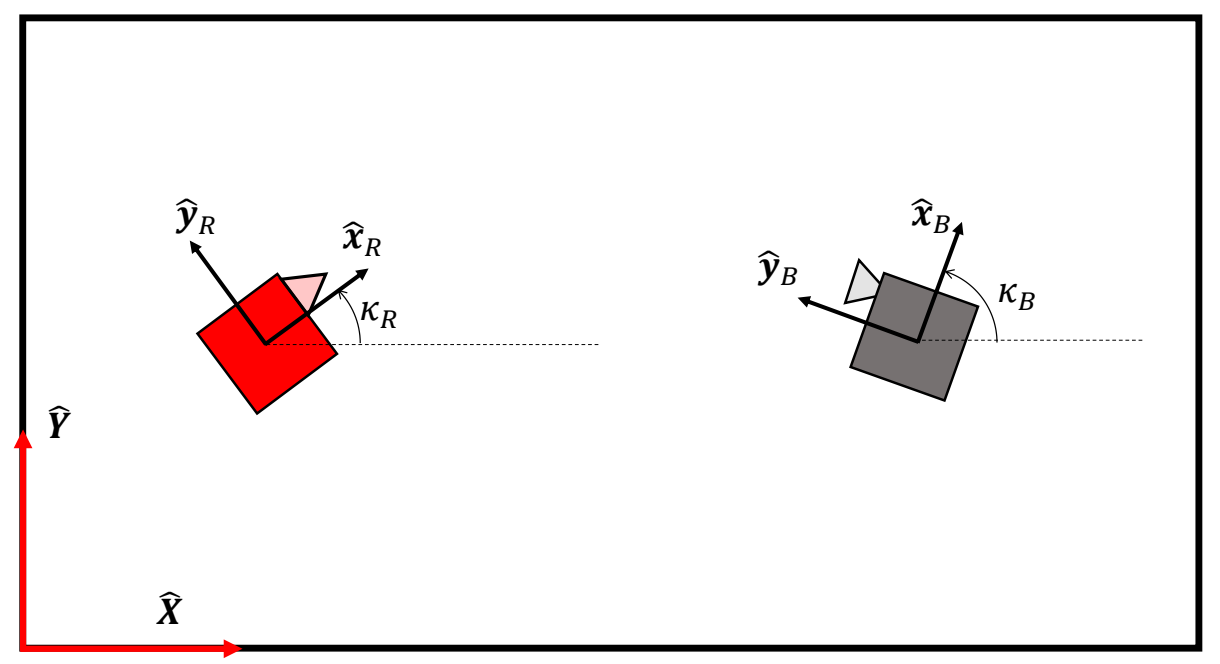

Figure 7.2: Definition of reference frames and attitude measurements.

Position and attitude measurements of each platform are made through the PhaseSpace motion capture system, which consists of eight cameras located above the granite table. These data are then wirelessly streamed to the platforms' on-board computers for real-time processing.

Both platforms use a Raspberry Pi 3 as the on-board flight-computer to handle attitude and translational control. The Raspberry Pi 3 has $4 \times$ ARM Cortex-A53, 1.2 GHz processors and 1 GB LPDDR2 RAM which, while more powerful than spacecraft flight-computers, will perform more realistically than the powerful processors contained in laptops. During experiment, each Raspberry Pi attains the relevant position and attitude data from the PhaseSpace system, performs the required onboard control algorithms, and then converts the combined thrust/torque output to Pulse Width Modulation values for each of the eight solenoid valves located on the platforms (two valves per vertical face).

\subsection{Experiment Scenario Definition}

For each scenario, RED will be considered the target and BLACK will be the chaser spacecraft. 


\subsubsection{Docking Geometry}

The required physical docking parameters for RED are tabulated in Table 7.1.

Table 7.1: RED docking geometric parameters.

\begin{tabular}{cccc}
\hline \hline \multicolumn{2}{c}{$\hat{\boldsymbol{o}}^{\prime B}$} & $\boldsymbol{d}^{B}(\mathrm{~m})$ & $\theta_{d}\left(^{\circ}\right)$ \\
\hline$\left[\begin{array}{lll}1 & 0 & 0\end{array}\right]^{T}$ & {$\left[\begin{array}{llll}0.4318 & 0 & 0\end{array}\right]^{T}$} & 30 \\
\hline \hline
\end{tabular}

The minimum safe-distance is taken to be $\alpha^{\prime}=0.20 \mathrm{~m}$. Geometrically, it is then found that $\alpha=0.7103 \mathrm{~m}$ and that the alignment vector for Phase 1 is $\hat{\boldsymbol{o}}^{B}=$ $\left[\begin{array}{lll}0.9743 & -0.2253 & 0\end{array}\right]^{T} \mathrm{~m}$.

\subsubsection{Parameter Optimization}

The method for obtaining the desired maximum attitude dynamics characteristics given by Eqs. (5.1)-(5.3) is considered beyond the scope of this work. Therefore, it is assumed that BLACK has perfect knowledge of the spin rate of RED for the parameter optimization process. For the interior point optimization, a value of $\Lambda=1000$ is used, and the constant weight set used is

$$
W_{t, P 1}=0.01 \quad W_{f, P 1}=1.0 \quad W_{\chi}=1 e-7 \quad W_{t, P 2}=0.01 \quad W_{f, P 2}=1.0
$$

These weights were selected specifically to promote more fuel-efficient experiments.

\subsubsection{Switching Conditions}

The BLACK platform is set to switch to Phase 2 guidance and control if the condition $\|\boldsymbol{r}-\alpha \hat{\boldsymbol{o}}\| \leq 5 \mathrm{~cm}$ is maintained for 5 seconds. The parameter $f$ in determining $r_{N}$ for the LVF is taken as $f=\frac{5}{6}$.

\subsubsection{Control Parameter}

The control parameter $\beta=0.2837 \mathrm{~s}^{-1}$ was chosen, which ensured that the velocity error dynamics were faster than the position dynamics of the maneuver during Phase 2 . 


\subsubsection{Initial Conditions}

For each experiment, the RED platform is given a five second period to reach the required angular rate before BLACK begins the docking maneuver. Five different initial condition sets are tested, and are tabulated in Table 7.2. These cases are specifically meant to test the performance of the docking algorithm under a variety of spin rates and initial alignment errors with $\hat{\boldsymbol{o}}$. Note that the values given for $\kappa_{R, 0}$ are angles before the 5 second spin-up phase, as the angle at the end of the phase is not controlled.

Table 7.2: Experiment initial conditions.

\begin{tabular}{|c|c|c|c|c|}
\hline Case & $\kappa_{R, 0}\left(^{\circ}\right)$ & $\omega_{R}(\operatorname{degs} / \mathrm{s})$ & \multicolumn{2}{|c|}{$\boldsymbol{r}_{0}(\mathrm{~m})$} \\
\hline 1 & 0 & 0.5 & {$[1.869$} & $-0.454]^{T}$ \\
\hline 2 & 72 & 2.0 & {$[1.913$} & $-0.595]^{T}$ \\
\hline 3 & 144 & -4.0 & {$[1.858$} & $-0.501]^{T}$ \\
\hline 4 & 216 & 6.0 & {$[1.840$} & $0.515]^{T}$ \\
\hline 5 & 144 & 8.0 & {$[1.946$} & $-0.465]^{T}$ \\
\hline
\end{tabular}

\subsection{Results}

Figures 7.3-7.7 show the acceleration constraints, convergence in $r, \theta, r_{d}, \theta^{\prime}$, and convergence of the actual velocity to the desired velocity for each initial condition considered. For the particular initial conditions cases 1, 3 and 5, time-lapses of the experiments are shown in Figs. 7.8-7.10 which help illustrate the behaviour at various target rotation speeds. For every case, as expected, successful docking occurred, and the parameter selection resulted in acceleration norms which were bounded by the constraint $u_{\max }$ except during initial error correction, or when noise momentarily causes a spike which goes beyond this bound. Likewise, the desired behaviour of convergence in $r$ followed by convergence in $\theta$ was observed in all cases, and convergence of $r_{d}$ to the docking position occurred in all cases while $\theta^{\prime}$ remained within acceptable bounds. It should be noted that when $r_{d}$ becomes small, the measurement $\theta^{\prime}$ becomes quite sensitive to position noise. This noisy angle measurement did not impede successful docking from occurring during any trials. 
Note that in case 5, a slight misalignment in the attitude of BLACK caused the mating attachments to initially "rattle" before the two spacecraft docked, leaving a small bump in the convergence of $r_{d}$ between approximately $170 \mathrm{~s}$ and $175 \mathrm{~s}$ in Fig. 7.4d.

The following are some notes regarding implementation of the CLVF-LVF strategy. Firstly because the control is dependent entirely on velocity- rather than positionerror, the controller output can be quite sensitive to measurement noise. In this work, the velocity data was attained through a numeric derivative running at $10 \mathrm{~Hz}$ while the remainder of the algorithm ran at $20 \mathrm{~Hz}$, and no filter was applied to this velocity computation. The inclusion of a Kalman filter (or another filtering technique) would likely become necessary in scenarios with significant corruption with noise.

Furthermore, note that there is a feed-forward acceleration term in the control law which is the time-derivative of the CLVF- or LVF- guidance. As shown in this thesis, making use of calculus, an analytical expression for this derivative can be obtained which is a function of the chaser position and velocity relative to the target. This analytical derivative is necessary for implementation, as otherwise, the desired velocity data includes far too much noise for the acceleration to be obtained numerically (this is quite apparent from Figs. $7.3 \mathrm{c}-7.7 \mathrm{c})$.

Overall, these results clearly indicate that the novel combined CLVF-LVF docking maneuver is suitable for real-time implementation. Importantly, the acceleration and path-constraints have been met by this algorithm in all cases without the need for heavy in-the-loop optimization. 


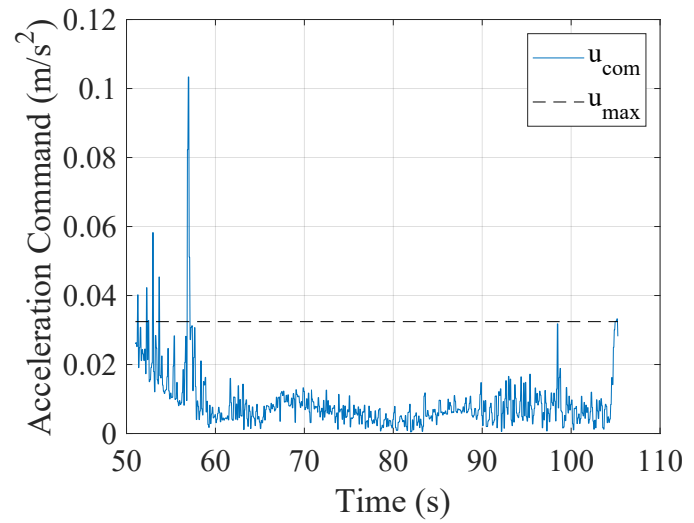

(a) Acceleration commands.
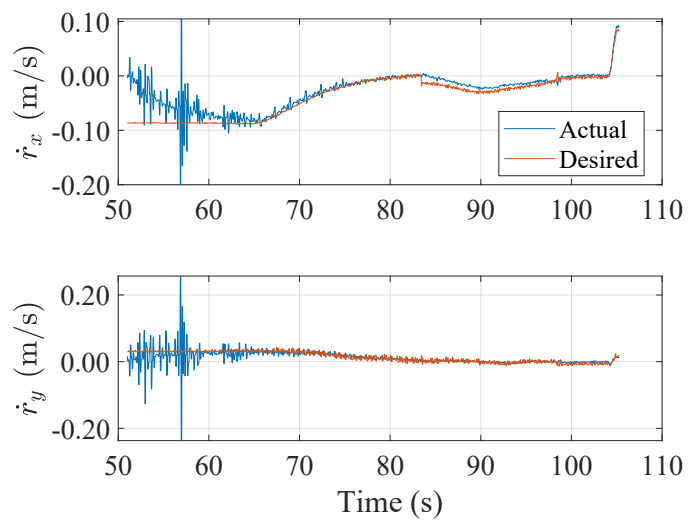

(c) Desired and actual velocity.
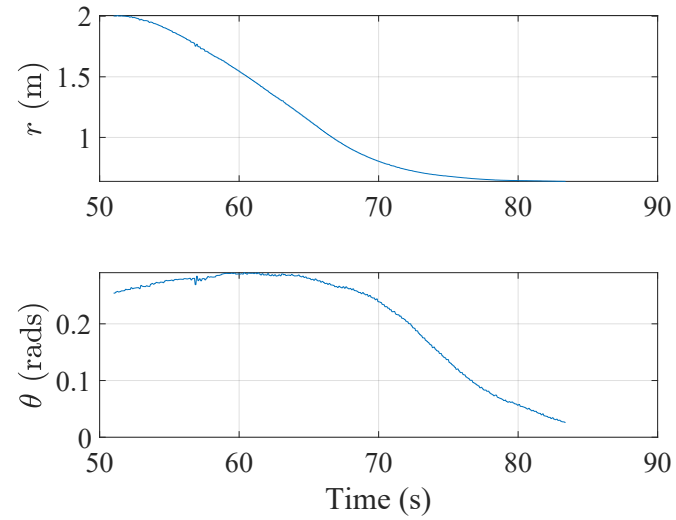

(b) Convergence of $r$ and $\theta$ over time.
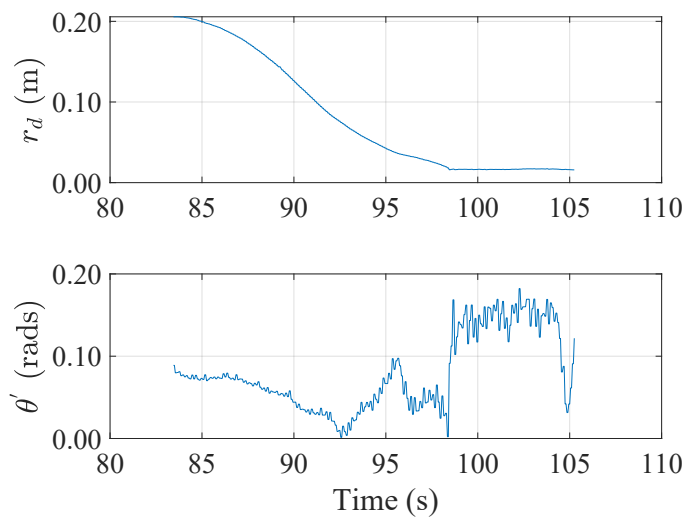

(d) Convergence of $r_{d}$ and $\theta^{\prime}$ over time.

Figure 7.3: Experimental results, initial condition case 1. 


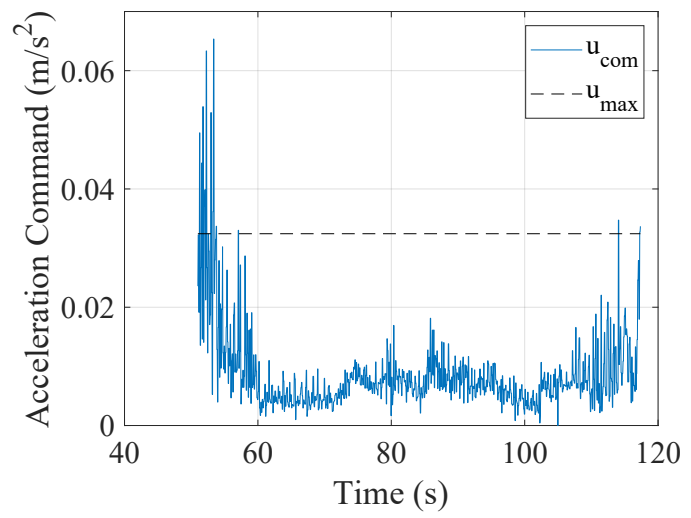

(a) Acceleration commands.
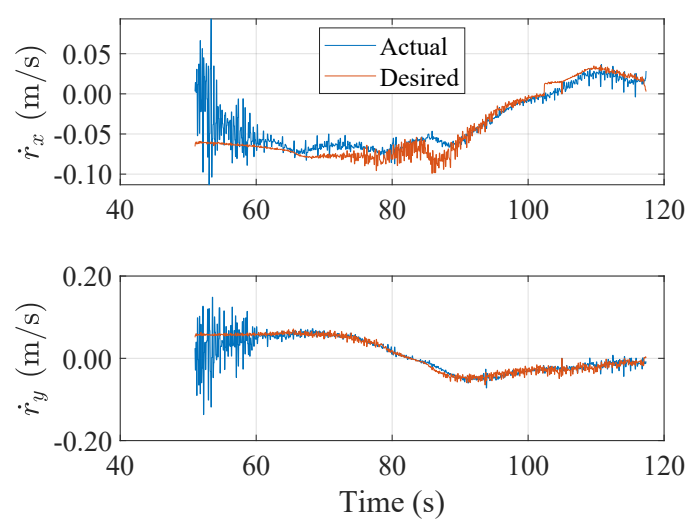

(c) Desired and actual velocity.
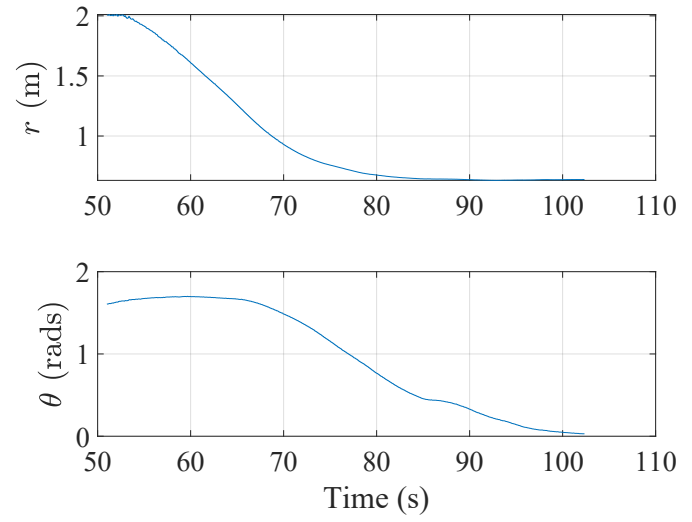

(b) Convergence of $r$ and $\theta$ over time.
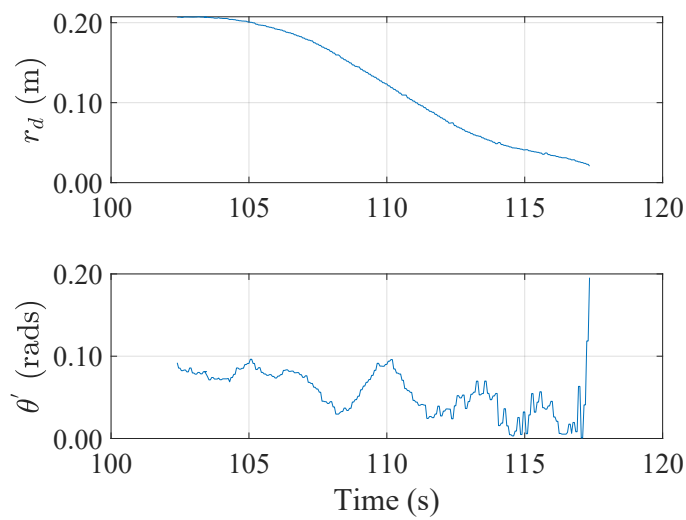

(d) Convergence of $r_{d}$ and $\theta^{\prime}$ over time.

Figure 7.4: Experimental results, initial condition case 2. 


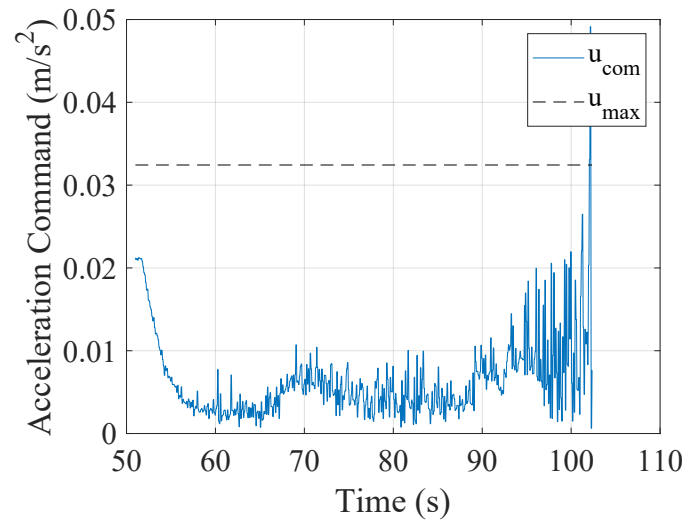

(a) Acceleration commands.
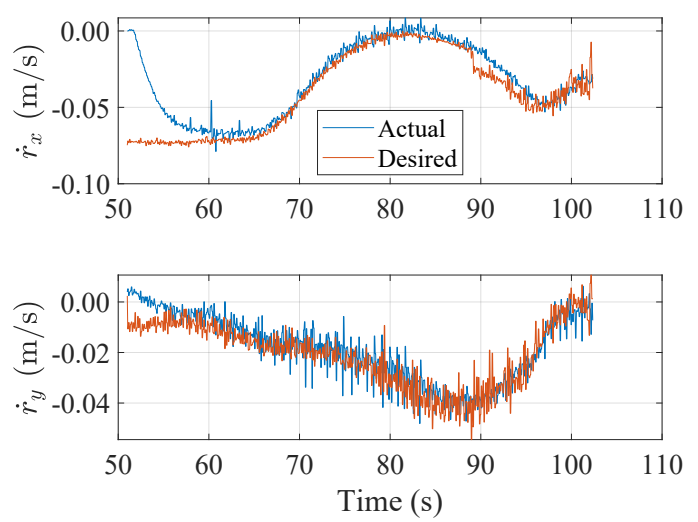

(c) Desired and actual velocity.
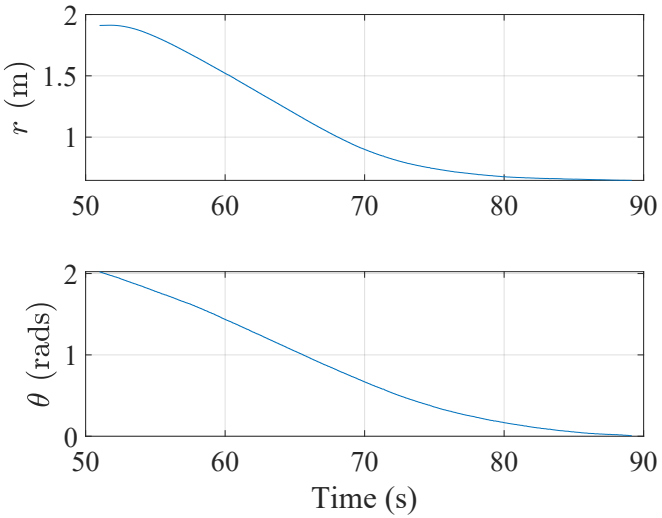

(b) Convergence of $r$ and $\theta$ over time.
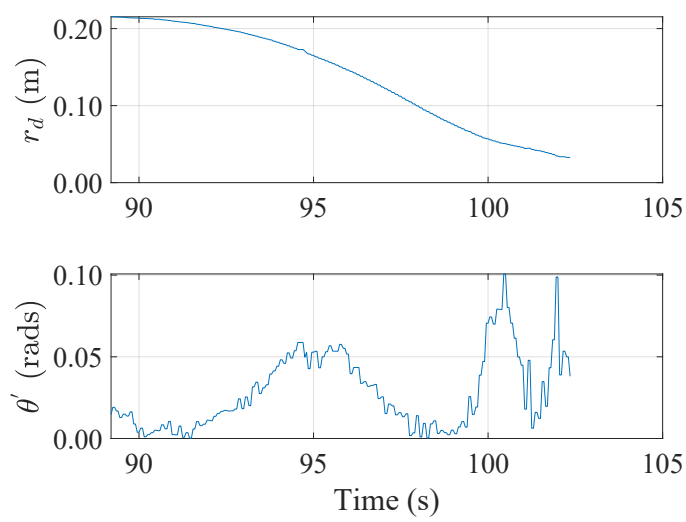

(d) Convergence of $r_{d}$ and $\theta^{\prime}$ over time.

Figure 7.5: Experimental results, initial condition case 3. 


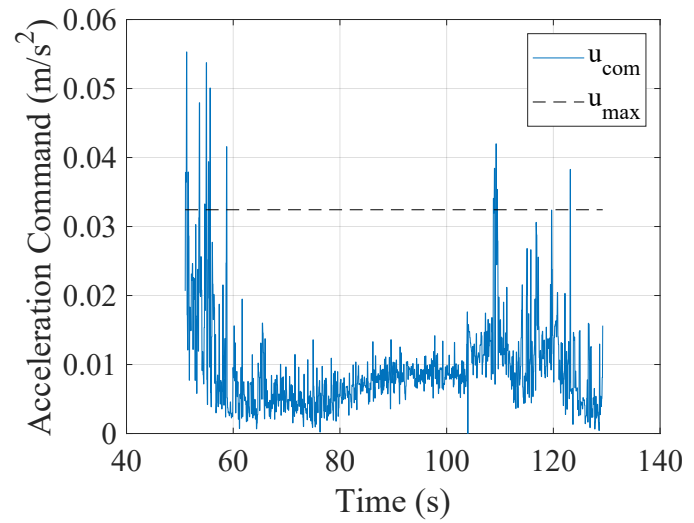

(a) Acceleration commands.
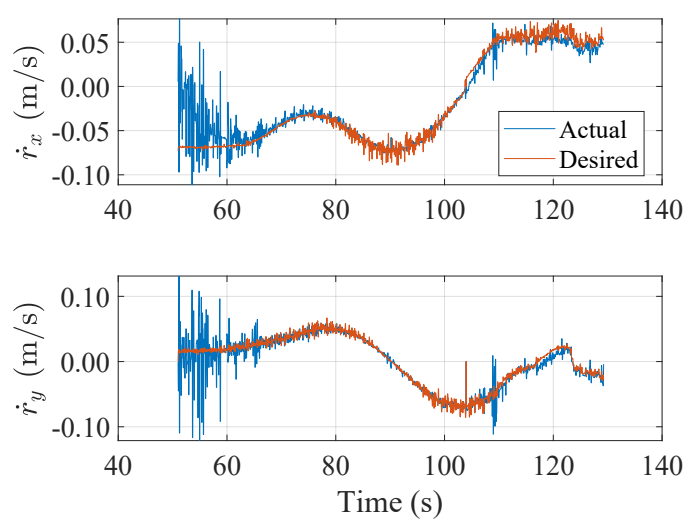

(c) Desired and actual velocity.
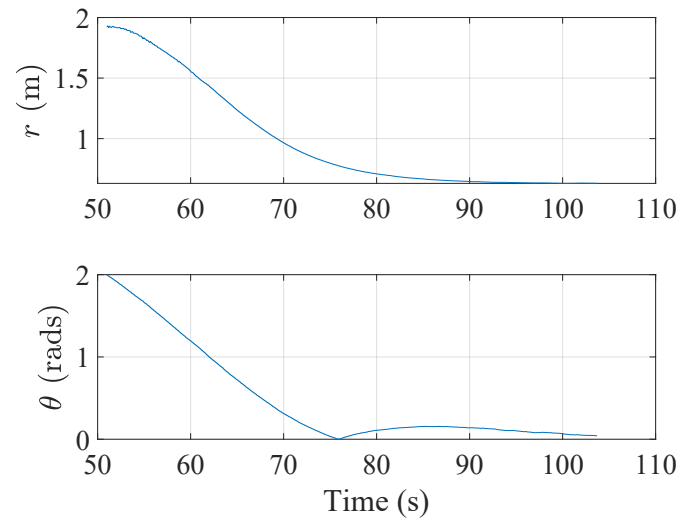

(b) Convergence of $r$ and $\theta$ over time.
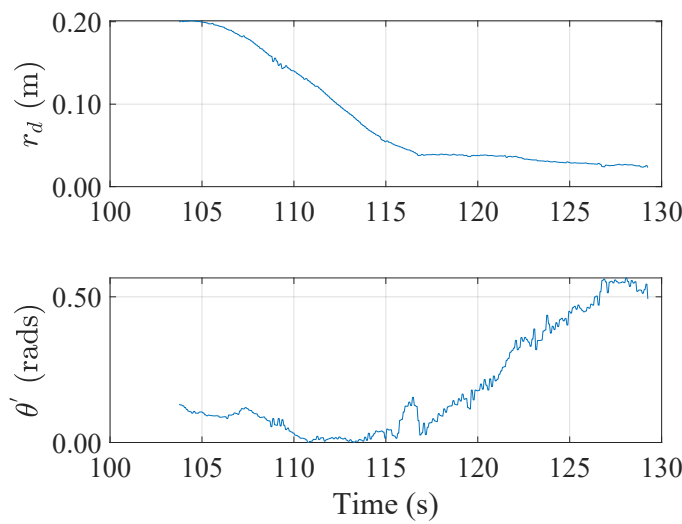

(d) Convergence of $r_{d}$ and $\theta^{\prime}$ over time.

Figure 7.6: Experimental results, initial condition case 4. 


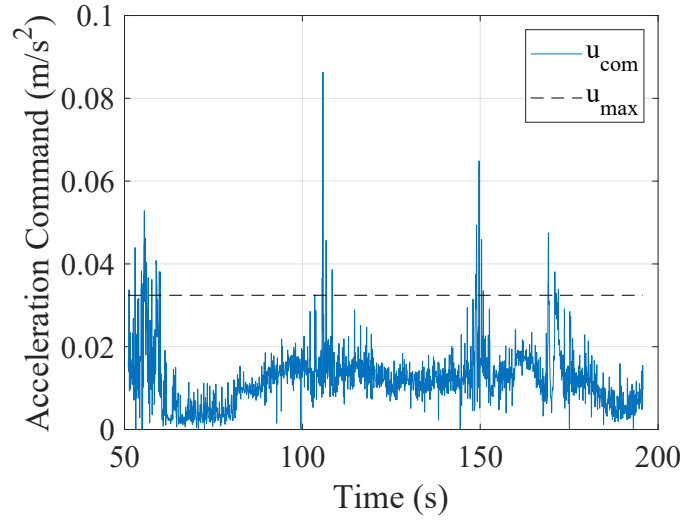

(a) Acceleration commands
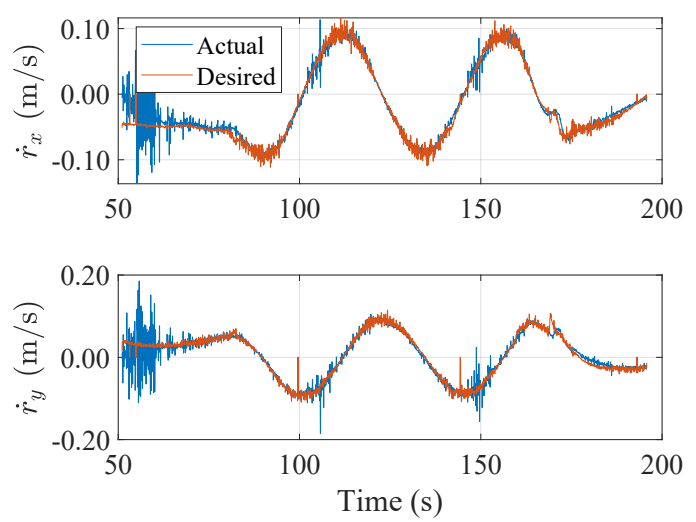

(c) Desired and actual velocity.
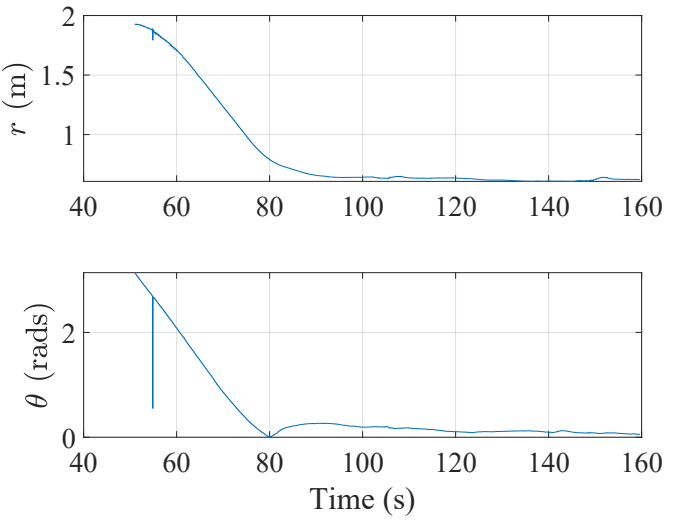

(b) Convergence of $r$ and $\theta$ over time.
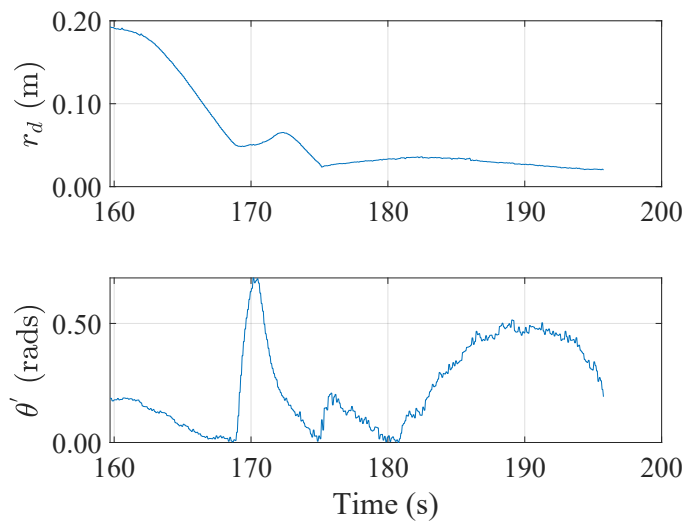

(d) Convergence of $r_{d}$ and $\theta^{\prime}$ over time.

Figure 7.7: Experimental results, initial condition case 5. 


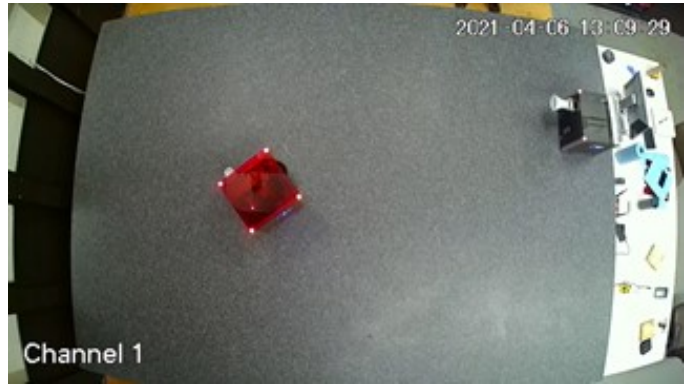

(a) Snapshot 1.

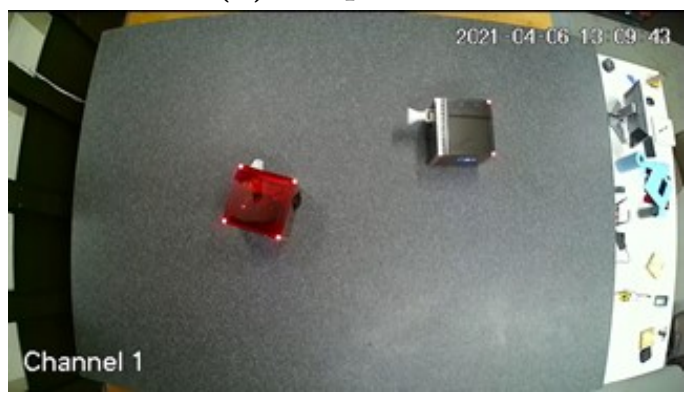

(c) Snapshot 3 .

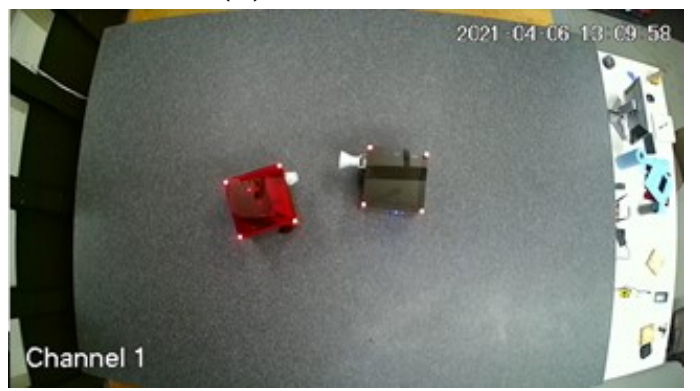

(e) Snapshot 5 .

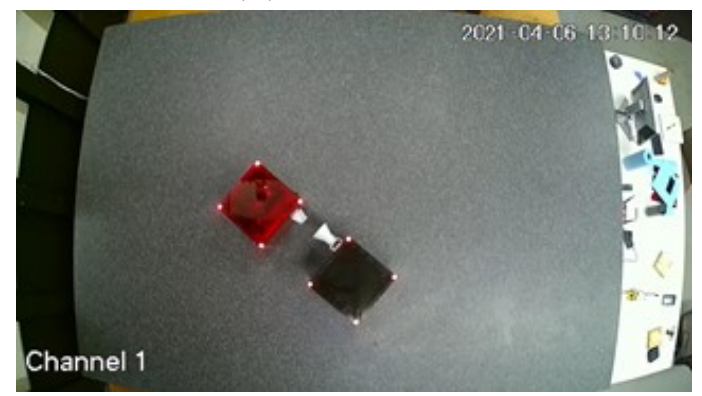

(g) Snapshot 7.

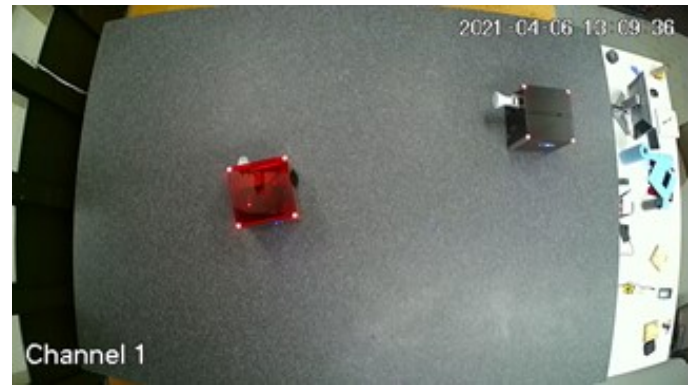

(b) Snapshot 2 .

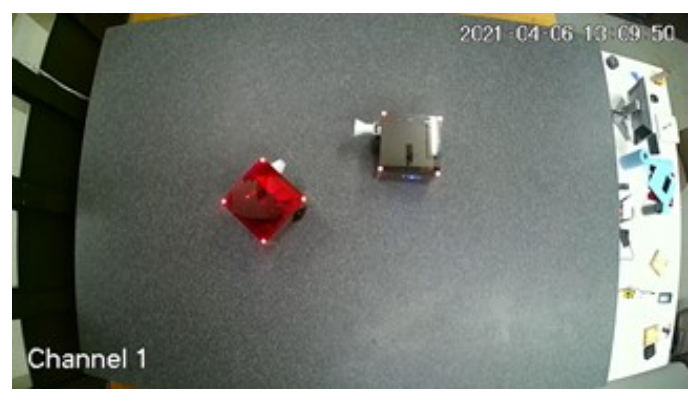

(d) Snapshot 4 .

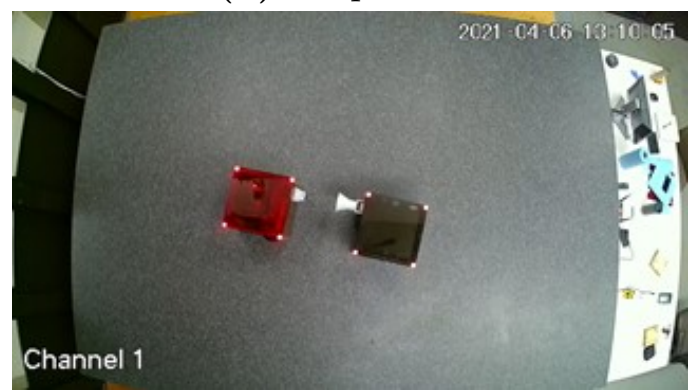

(f) Snapshot 6 .

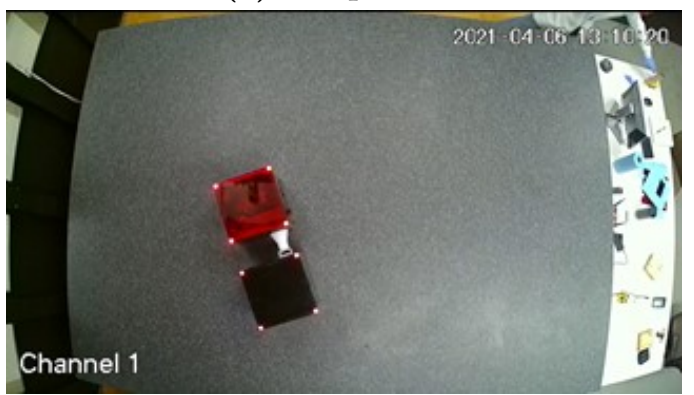

(h) Snapshot 8.

Figure 7.8: Initial condition set 1, timelapse. 


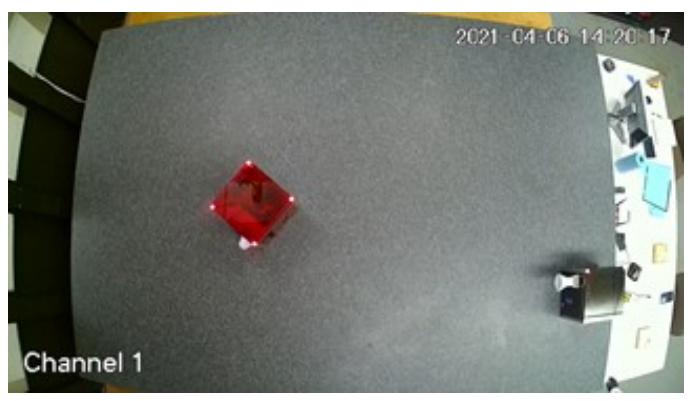

(a) Snapshot 1.

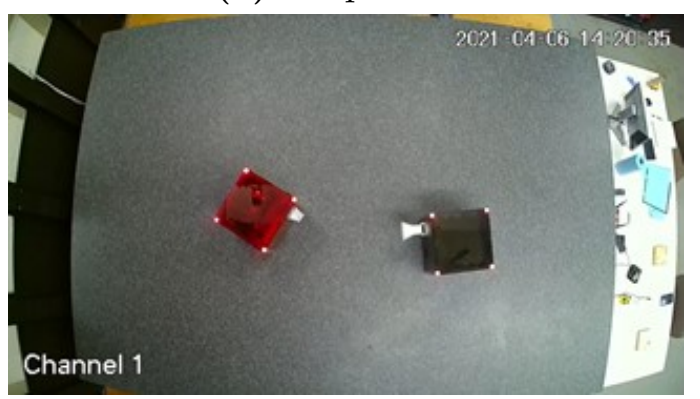

(c) Snapshot 3.

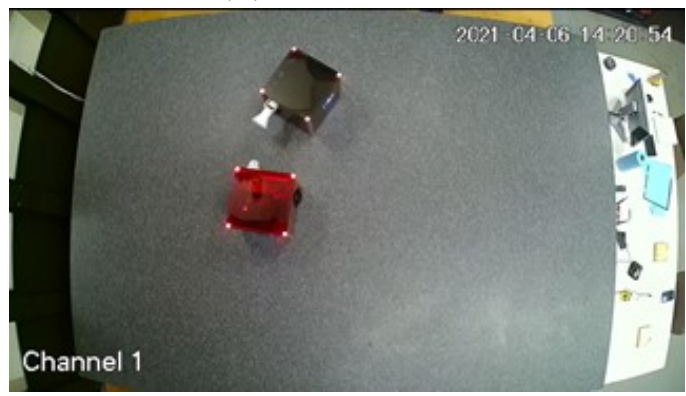

(e) Snapshot 5 .

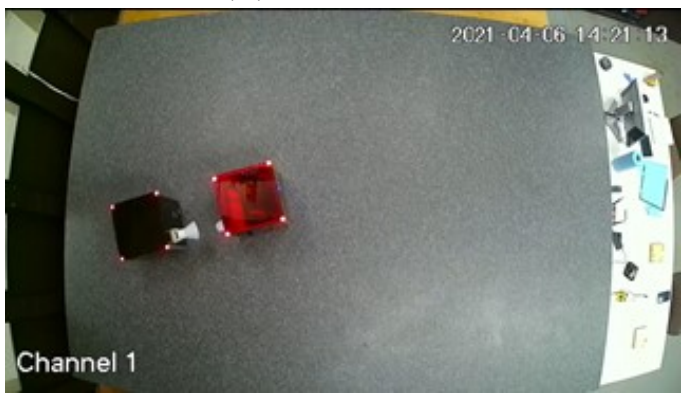

(g) Snapshot 7.

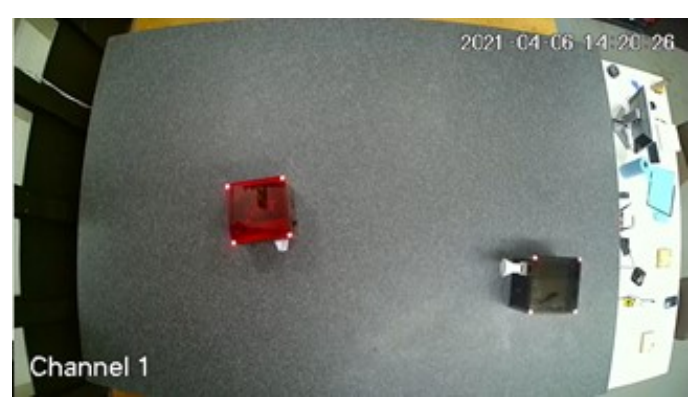

(b) Snapshot 2 .

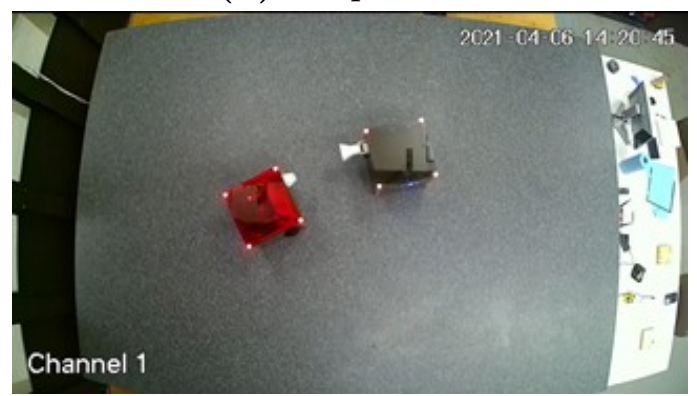

(d) Snapshot 4 .

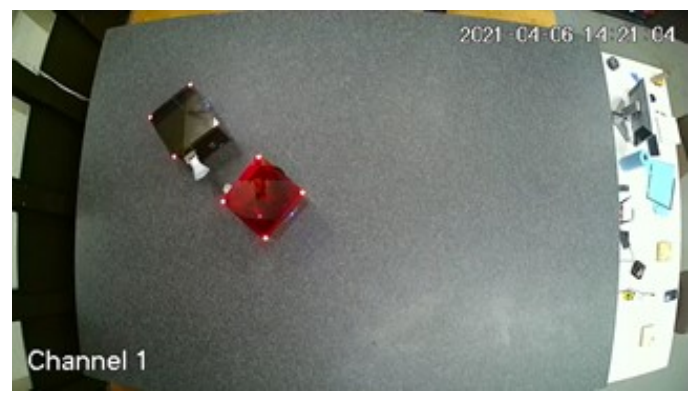

(f) Snapshot 6 .

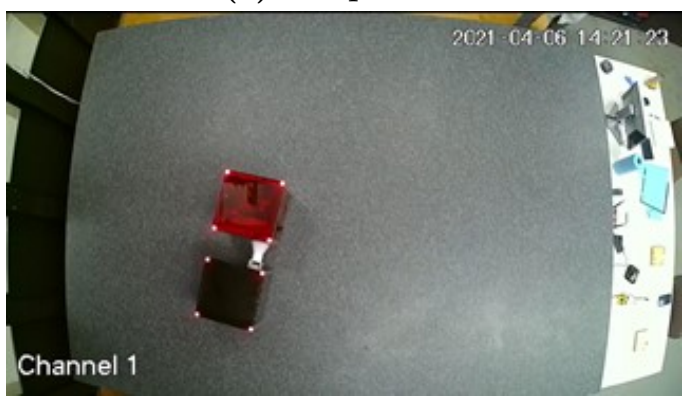

(h) Snapshot 8.

Figure 7.9: Initial condition set 3, timelapse. 


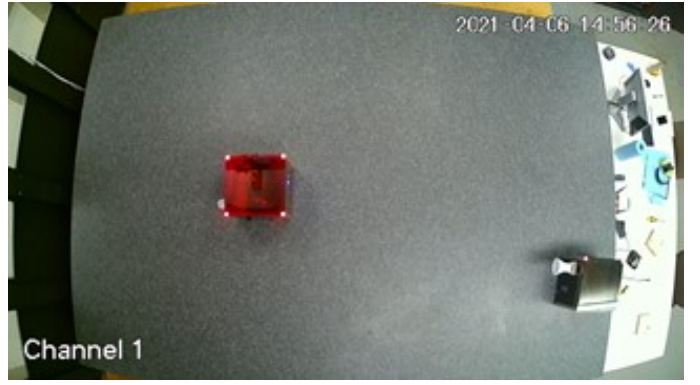

(a) Snapshot 1.

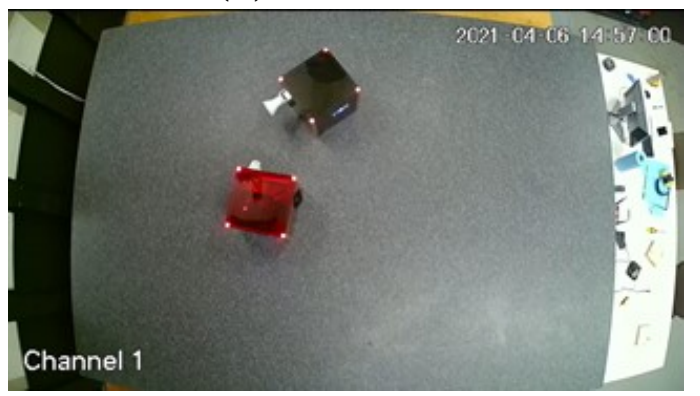

(c) Snapshot 3 .

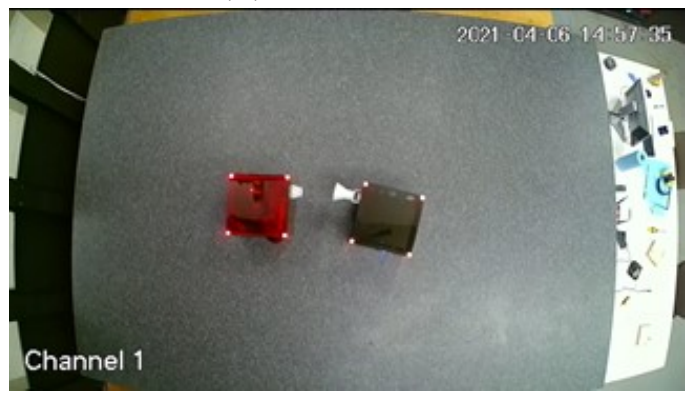

(e) Snapshot 5 .

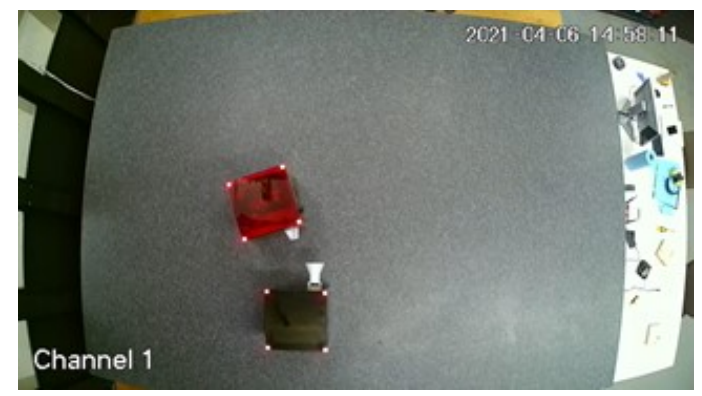

(g) Snapshot 7.

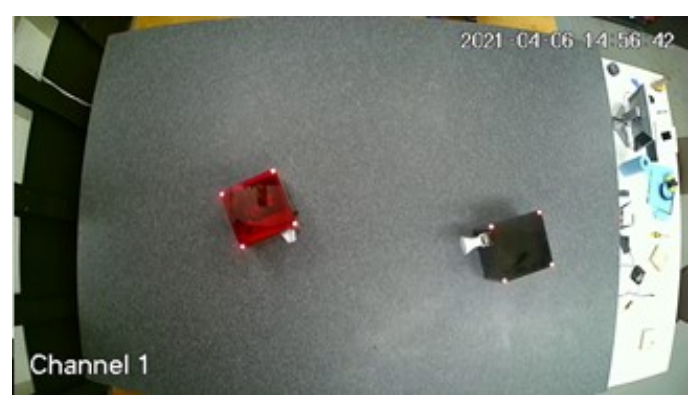

(b) Snapshot 2 .

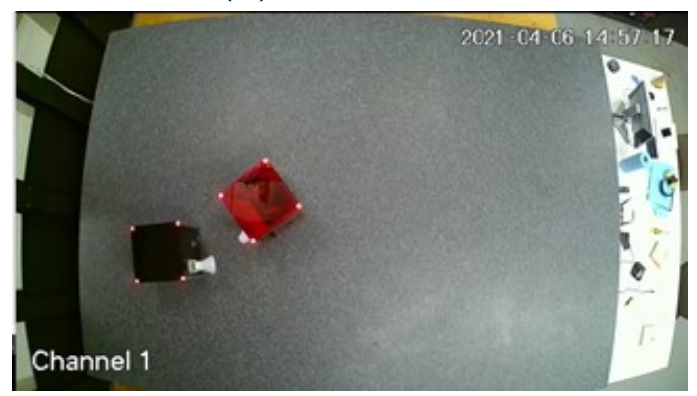

(d) Snapshot 4 .

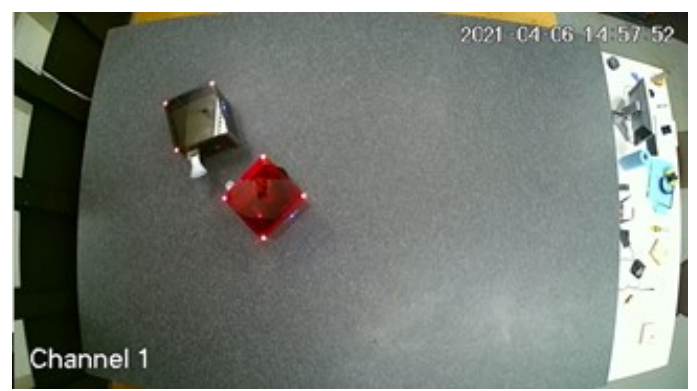

(f) Snapshot 6 .

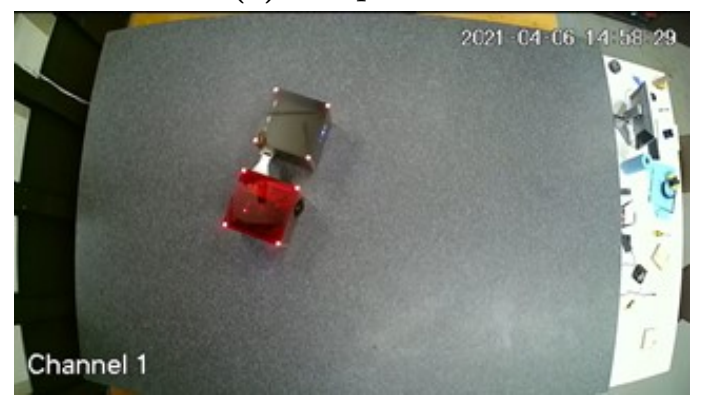

(h) Snapshot 8 .

Figure 7.10: Initial condition set 5, timelapse. 


\section{Chapter 8}

\section{Conclusions}

This Chapter begins by summarizing the work completed in this thesis in Sec. 8.1. Some improvements and future research directions are then discussed in Sec. 8.2. Finally, some notes regarding publications which have resulted from (or are being prepared base on) this work are provided in Sec. 8.3

\subsection{Thesis Summary}

In this thesis, a novel method for low-computation spacecraft docking based on a Lyapunov vector field framework has been presented. To achieve this, a substantial extension to the Lyapunov vector field (herein referred to as a cascaded Lyapunov vector field) was developed which broadens the applications of LVFs to cases where trajectories are defined in generally tumbling and accelerating reference frames. Using this extension, a new CLVF-LVF two phase docking maneuver was designed which includes inherently stable guidance, and obeys the docking path constraints by construction. Furthermore, convex sets of field parameters (i.e., $k_{a}, k_{c}, b, \alpha^{\prime}$ and $v_{\max }$ ) were derived such that acceleration constraints are guaranteed to be respected when tracking the CLVF- or LVF-guidance. An approach to performance optimization within the CLVF-LVF framework has been presented based on maneuver fuel and time estimates, and this method is shown to work reliably for lower target angular velocities, which are very common in practice. Provided that there is limited disparity in the fuel and time weights, the parameter optimization process developed in this thesis also successfully optimizes the performance for cases with higher target angular velocities, although this requires additional improvements and study.

Importantly, this work considered path constraints, acceleration constraints, and performance optimization each without the requirement for heavy in-the loop optimizations, which conserves the real-time computational feasibility of this approach 
on-board a spacecraft flight computer.

The docking approach developed in this thesis was validated in real-time experiments, showing robustness to disturbances such as imperfect thrust resolution, timedelays, measurement noise, and perturbations such as air-resistance.

\subsection{Improvements and Future Work}

The author identifies several areas of future research regarding the CLVF-LVF approach developed in this thesis.

Primarily, the approach for parameter optimization in Phase 1 requires further improvements and additional study. The present robustness issues appear to stem from an inaccurate estimation of required acceleration to track the CLVF when the target tumbles at relatively high angular velocities ( $\geq 3 \mathrm{degs} / \mathrm{s})$. Improving this estimate would likely increase the robustness of the optimization, making it more suitable for use in spacecraft where significant algorithm variability and risk are not acceptable.

Secondly, a system which takes the attitude of the chaser into consideration is required to decrease the risk of this maneuver. While it is unnecessary to fully control the attitude of the chaser within the framework presented here (most spacecraft in practice have independent translation and attitude control systems), there is presently no safety mechanism in place should attitude misalignments occur just prior to contact (indeed, one such scenario was observed in experiment). While this is considered beyond the scope of what was presented here, it is nonetheless a necessary addition if this algorithm is to be implemented on-board a real mission.

Another area of future research is regarding improvement to the loose upper-bound on the required acceleration for the Phase 2 LVF guidance. The implications of this improvement would be two-fold: first, the parameter optimization of the Phase 2 maneuver is presently restricted in performance because the region of feasible parameters is overly-constrained. By finding a tighter acceleration bound, the optimization could further exploit the LVF parameters. Further, this increased flexibility in parameter selection would allow the chaser spacecraft to make more significant use of the full acceleration capabilities in Phase 2, should this be deemed desirable.

In addition, it may be quite interesting to explore adaptive field parameters in 
future work. Field parameters which adapt over time may include several benefits not achieved in this work. For example, this thesis assumes constant mass for the chaser spacecraft. Under this assumption, a constant thrust limitation corresponds to a constant acceleration limit. In reality, the mass (and by extension, the acceleration constraints) continuously change as fuel is expelled. Adaptive field parameters might be a natural selection to continue to exploit acceleration limits, even as they change over time.

\subsection{Significance of Work}

The importance of this work is evidenced by the following publication, which was awarded Best Presentation in Session.

Hough, J.G., and Ulrich, S., "Lyapunov Vector Fields for Thrust-Limited Spacecraft Docking with an Elliptically-Orbiting Uncooperative Tumbling Target" AIAA Guidance, Navigation, and Control Conference, Orlando, FL, 6-10 Jan, 2020. Best Presentation in Session.

Furthermore, the novel development of cascaded Lyapunov vector fields is presently under review as a manuscript for the AIAA Journal of Guidance, Dynamics and Control. 


\section{Appendix A}

\section{Evaluation of the CLVF robustness}

To evaluate the robustness of convergence errors, let us begin with Eq. (3.55)

$$
\Delta \theta \leq\left(\frac{\left\|\frac{\partial \psi}{\partial \boldsymbol{r}}\left(\boldsymbol{r}^{*}, \boldsymbol{o}, \cdot\right)\right\|}{\alpha\left\|\frac{\partial V_{a}}{\partial \boldsymbol{r}}\left(\boldsymbol{r}^{*}, \boldsymbol{o}, \cdot\right) \frac{\partial \boldsymbol{a}}{\partial \boldsymbol{r}}\left(\boldsymbol{r}^{*}, \boldsymbol{o}, \cdot\right)\right\|}\right)\left\|\Delta \boldsymbol{r}_{n}\right\|
$$

where Eqs. (3.36) and (3.38) with the identity Eq. (3.67) simplifies the error function to

$$
\psi\left(\boldsymbol{r}, \hat{\boldsymbol{o}}, \boldsymbol{\omega}_{O I}\right)=\frac{d V_{a}}{d \theta}\left(\boldsymbol{\omega}_{O I}^{T} \hat{\boldsymbol{e}}\right)\left(1-\frac{g(r)}{r}\right)
$$

Note that when $r \leq \alpha, \phi \equiv 0$. Therefore, there are only robustness concerns if the convergence error pushes the spacecraft outside of the radius $\alpha$. Evaluating $\frac{\partial \psi}{\partial \boldsymbol{r}}$ and substituting Eq. (3.115) when $r>\alpha$ for $g(r)$

$\frac{\partial \psi}{\partial \boldsymbol{r}}=\frac{d^{2} V_{a}}{d \theta^{2}} \frac{\partial \theta}{\partial \boldsymbol{r}}\left(\boldsymbol{\omega}_{O I}^{T} \hat{\boldsymbol{e}}\right)\left(1-\frac{\alpha^{2}}{r^{2}}\right)+\frac{d V_{a}}{d \theta} \frac{\partial}{\partial \boldsymbol{r}}\left(\boldsymbol{\omega}_{O I}^{T} \hat{\boldsymbol{e}}\right)\left(1-\frac{\alpha^{2}}{r^{2}}\right)+\frac{d V_{a}}{d \theta}\left(\boldsymbol{\omega}_{O I}^{T} \hat{\boldsymbol{e}}\right)\left(2 \frac{\alpha^{2}}{r^{3}} \hat{\boldsymbol{r}}^{T}\right)$

Evaluating this function at an equilibrium point (i.e. $r=\alpha$ ) and recalling that $\left\|\frac{\partial \theta}{\partial \boldsymbol{r}}\right\|=\frac{1}{r}$

$$
\frac{\partial \psi}{\partial \boldsymbol{r}}\left(\boldsymbol{r}^{*}, \hat{\boldsymbol{o}}, \boldsymbol{\omega}_{O I}\right)=\frac{d V_{a}}{d \theta}\left(\boldsymbol{\omega}_{O I}^{T} \hat{\boldsymbol{e}}\right)\left(\frac{2 \hat{\boldsymbol{r}}^{T}}{\alpha}\right)
$$

which has a norm bounded by

$$
\left\|\frac{\partial \psi}{\partial \boldsymbol{r}}\left(\boldsymbol{r}^{*}, \hat{\boldsymbol{o}}, \boldsymbol{\omega}_{O I}\right)\right\| \leq \frac{2 \omega_{\max }}{\alpha} \frac{d V_{a}}{d \theta}
$$

Similarly, to find the denominator of Eq. (3.55) we first find the general Jacobian $\frac{\partial \boldsymbol{a}}{\partial \boldsymbol{r}}$ after substituting Eq. (3.117) for $s_{a}(r, \theta)$. However, after substituting $\theta=0$ (or 
$\theta=\pi$ ) when evaluating the Jacobian at $\boldsymbol{r}^{*}$, many terms go to zero only leaving

$$
\frac{\partial \boldsymbol{a}}{\partial \boldsymbol{r}}\left(\boldsymbol{r}^{*}, \boldsymbol{o}\right)=k_{a} \frac{\alpha}{r} \cos \theta \hat{\boldsymbol{a}} \frac{\partial \theta}{\partial \boldsymbol{r}}+k_{a} \frac{\alpha}{r} \sin \theta \frac{\partial \hat{\boldsymbol{a}}}{\partial \boldsymbol{r}}
$$

where it is not immediately clear whether the last term goes to $\mathbf{0}$. Evaluating $\frac{\partial \hat{\boldsymbol{a}}}{\partial \boldsymbol{r}}$ for $(0<\theta<\pi)$ and simplifying using Eqs. (3.64), (3.65) and (3.71) results in

$$
\frac{\partial \hat{\boldsymbol{a}}}{\partial \boldsymbol{r}}=-\frac{\hat{\boldsymbol{r}} \hat{\boldsymbol{a}}^{T}}{r}-\frac{\cos \theta}{\sin \theta} \frac{\hat{\boldsymbol{e}} \hat{\boldsymbol{e}}^{T}}{r}
$$

The terms within the norm in the denominator of Eq. (3.55) then become

$$
\frac{\partial V_{a}}{\partial \boldsymbol{r}}\left(\boldsymbol{r}^{*}, \boldsymbol{o}, \cdot\right) \frac{\partial \boldsymbol{a}}{\partial \boldsymbol{r}}\left(\boldsymbol{r}^{*}, \boldsymbol{o}, \cdot\right)=\frac{\partial V_{a}}{\partial \boldsymbol{r}}\left(\boldsymbol{r}^{*}, \boldsymbol{o}, \cdot\right)\left(k_{a} \frac{\alpha}{r} \cos \theta \hat{\boldsymbol{a}} \frac{\partial \theta}{\partial \boldsymbol{r}}+k_{a} \frac{\alpha}{r} \sin \theta\left(-\frac{\hat{\boldsymbol{r}} \hat{\boldsymbol{a}}^{T}}{r}-\frac{\cos \theta}{\sin \theta} \frac{\hat{\boldsymbol{e}} \hat{\boldsymbol{e}}^{T}}{r}\right)\right)
$$

Noting that

$$
\frac{\partial V_{a}}{\partial \boldsymbol{r}} \hat{\boldsymbol{r}}=\frac{\partial V_{a}}{\partial \boldsymbol{r}} \hat{\boldsymbol{e}}=0
$$

the denominator is then

$\alpha\left\|\frac{\partial V_{a}}{\partial \boldsymbol{r}}\left(\boldsymbol{r}^{*}, \boldsymbol{o}, \cdot\right) \frac{\partial \boldsymbol{a}}{\partial \boldsymbol{r}}\left(\boldsymbol{r}^{*}, \boldsymbol{o}, \cdot\right)\right\|=\alpha\left\|\frac{d V_{a}}{d \theta}\left(\frac{\partial \theta}{\partial \boldsymbol{r}} \hat{\boldsymbol{a}}\right) k_{a} \frac{\alpha}{r} \cos \theta \frac{\partial \theta}{\partial \boldsymbol{r}}\right\|=\alpha\left\|\frac{d V_{a}}{d \theta}\left(\frac{-1}{r}\right) k_{a} \frac{\alpha}{r} \cos \theta \frac{1}{r}\right\|$

which, evaluating at $r=\alpha$ and $\|\cos \theta\|=1$, simplifies to

$$
\alpha\left\|\frac{\partial V_{a}}{\partial \boldsymbol{r}}\left(\boldsymbol{r}^{*}, \boldsymbol{o}, \cdot\right) \frac{\partial \boldsymbol{a}}{\partial \boldsymbol{r}}\left(\boldsymbol{r}^{*}, \boldsymbol{o}, \cdot\right)\right\|=\frac{d V_{a}}{d \theta} \frac{k_{a}}{\alpha}
$$

Therefore, evaluating Eq. (3.55) for the selected $s_{a}(r, \theta)$ and $g(r)$ functions leads to the following robustness equation

$$
\frac{\Delta \theta}{\left\|\Delta \boldsymbol{r}_{n}\right\|} \leq \frac{2 \omega_{\max }}{k_{a}}
$$




\section{Appendix B}

\section{Conversion from Orbital Elements to Initial ECI Position and Velocity}

Note that the following steps can be found in any textbook covering orbital mechanics. One example is [10].

To begin, define the following distance $p$ as the semi-latus rectum

$$
p=œ_{a}\left(1-œ_{e}^{2}\right)
$$

the distance from the Earth's center of mass is then given by

$$
R=\frac{p}{1+œ_{e} \cos \left(œ_{\theta}\right)}
$$

After defining this distance, let us define the perifocal reference frame as having an origin at Earth's center of mass, a z-vector $\hat{\boldsymbol{P}}_{z}$ parallel to the orbit angular momentum vector, and an x-vector $\hat{\boldsymbol{P}}_{x}$ which points to perigee. The position vector in the perifocal frame is then given by

$$
\boldsymbol{R}^{P}=\left[\begin{array}{c}
R \cos \left(œ_{\theta}\right) \\
R \sin \left(œ_{\theta}\right) \\
0
\end{array}\right]
$$

Finally, note that the perifocal frame can be reached from the ECI frame by the following 3-1-3 rotation sequence

$$
\boldsymbol{C}_{P I}=\boldsymbol{C}_{z}\left(œ_{\omega}\right) \boldsymbol{C}_{x}\left(œ_{i}\right) \boldsymbol{C}_{z}\left(\wp_{\Omega}\right)
$$


where, for an arbitrary angle $\psi$

$$
\begin{aligned}
& \boldsymbol{C}_{z}(\psi)=\left[\begin{array}{ccc}
\cos \psi & \sin \psi & 0 \\
-\sin \psi & \cos \psi & 0 \\
0 & 0 & 1
\end{array}\right] \\
& \boldsymbol{C}_{x}(\psi)=\left[\begin{array}{ccc}
1 & 0 & 0 \\
0 & \cos \psi & \sin \psi \\
0 & -\sin \psi & \cos \psi
\end{array}\right]
\end{aligned}
$$

The position vector in ECI is then given by

$$
\boldsymbol{R}=\boldsymbol{C}_{P I}^{T} \boldsymbol{R}^{P}
$$

Furthermore, by the velocity in the perifocal frame is given by

$$
\dot{\boldsymbol{R}}^{P}=\left[\begin{array}{c}
-\sqrt{\frac{\mu}{p}} \sin œ_{\theta} \\
\sqrt{\frac{\mu}{p}} \cos œ_{\theta} \\
0
\end{array}\right]
$$

Finally, the velocity in ECI is obtained through a rotation

$$
\dot{\boldsymbol{R}}=\boldsymbol{C}_{P I}^{T} \dot{\boldsymbol{R}}^{P}
$$




\section{Appendix C}

\section{Gradient and Hessian of Cost Functions}

First, noting that the cost function is of the form

$$
C=\lambda J+P
$$

The gradient and hessian in both cases will have the following structure

$$
\begin{gathered}
\frac{d C}{d \boldsymbol{p}}=\lambda \frac{d J}{d \boldsymbol{p}}+\frac{d P}{d \boldsymbol{p}} \\
\frac{d^{2} C}{d \boldsymbol{p}^{2}}=\lambda \frac{d^{2} J}{d \boldsymbol{p}^{2}}+\frac{d^{2} P}{d \boldsymbol{p}^{2}}
\end{gathered}
$$

Noting that in this thesis, the penalty term $P$ is selected as a log-barrier function on a slack-vector $s$

$$
\begin{gathered}
\frac{d P}{d \boldsymbol{p}}=-\sum_{i=1}^{M} \frac{1}{s_{i}} \frac{d s_{i}}{d \boldsymbol{p}} \\
\frac{d^{2} P}{d \boldsymbol{p}^{2}}=\sum_{i=1}^{M}\left(\frac{d s_{i}{ }^{T}}{d \boldsymbol{p}} \frac{1}{s_{i}^{2}} \frac{d s_{i}}{d \boldsymbol{p}}-\frac{1}{s_{i}} \frac{d^{2} s_{i}}{d \boldsymbol{p}^{2}}\right)
\end{gathered}
$$

For any entries of the slack vectors which are included to enforce positive parameter selections (i.e. all but the last entry in both slack vectors)

$$
\frac{d s_{i}}{d p_{j}}= \begin{cases}1, & \text { for } i=j \\ 0, & \text { otherwise }\end{cases}
$$

and all Hessian entries will be 0 , since this part of the slack vector is affine. For the last entry in each slack vector

$$
\frac{d s_{M}}{d \boldsymbol{p}}=-\frac{d}{d \boldsymbol{p}} u_{P, i}(\boldsymbol{p})
$$




$$
\frac{d^{2} s_{M}}{d \boldsymbol{p}^{2}}=-\frac{d^{2}}{d \boldsymbol{p}^{2}} u_{P, i}(\boldsymbol{p})
$$

Using MATLAB symbolic, these expressions are found for Phase 1 as

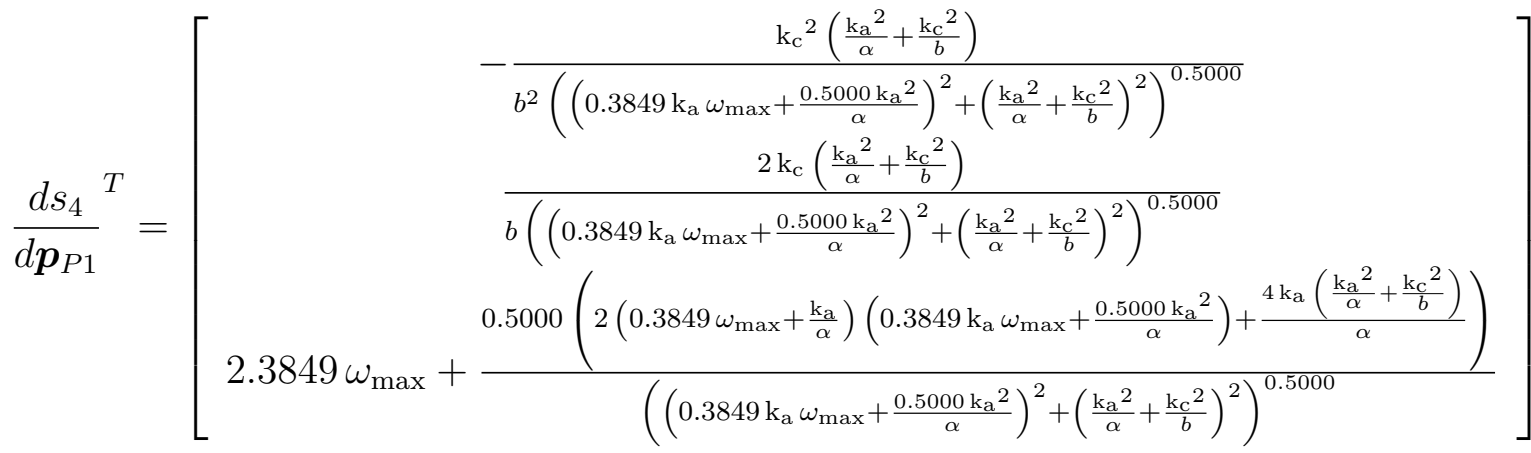

$$
\begin{aligned}
& \frac{d^{2} s_{4}}{d \boldsymbol{p}_{P 1}^{2}}=\left[\begin{array}{lll}
e_{11} & e_{12} & e_{13} \\
e_{21} & e_{22} & e_{23} \\
e_{31} & e_{32} & e_{33}
\end{array}\right]
\end{aligned}
$$

where 


$$
\begin{aligned}
& e_{11}=\frac{q_{11}}{\alpha^{3} b^{6}\left(\left(0.3849 \mathrm{k}_{\mathrm{a}} \omega_{\max }+\frac{0.5000 \mathrm{k}_{\mathrm{a}}{ }^{2}}{\alpha}\right)^{2}+\left(\frac{\mathrm{k}_{\mathrm{a}}{ }^{2}}{\alpha}+\frac{\mathrm{k}_{\mathrm{c}}{ }^{2}}{b}\right)^{2}\right)^{1.5000}} \\
& q_{11}=0.0093 \mathrm{k}_{\mathrm{c}}{ }^{2}\left(48 \alpha^{3} b^{2} \mathrm{k}_{\mathrm{a}}{ }^{2} \mathrm{k}_{\mathrm{c}}{ }^{2} \omega_{\max }{ }^{2}+216 \alpha^{3} \mathrm{k}_{\mathrm{c}}{ }^{6}+32 \alpha^{2} b^{3} \mathrm{k}_{\mathrm{a}}{ }^{4}\right. \\
& \omega_{\max }^{2}+124.7077 \alpha^{2} b^{2} \mathrm{k}_{\mathrm{a}}^{3} \mathrm{k}_{\mathrm{c}}{ }^{2} \omega_{\max }+648 \alpha^{2} b \mathrm{k}_{\mathrm{a}}^{2} \\
& \left.\mathrm{k}_{\mathrm{c}}{ }^{4}+83.1384 \alpha b^{3} \mathrm{k}_{\mathrm{a}}{ }^{5} \omega_{\max }+729 \alpha b^{2} \mathrm{k}_{\mathrm{a}}{ }^{4} \mathrm{k}_{\mathrm{c}}{ }^{2}+270 b^{3} \mathrm{k}_{\mathrm{a}}{ }^{6}\right) \\
& e_{12}=-\frac{q_{12}}{\alpha^{3} b^{5}\left(\left(0.3849 \mathrm{k}_{\mathrm{a}} \omega_{\max }+\frac{0.5000 \mathrm{k}_{\mathrm{a}}^{2}}{\alpha}\right)^{2}+\left(\frac{\mathrm{k}_{\mathrm{a}}^{2}}{\alpha}+\frac{\mathrm{k}_{\mathrm{c}}{ }^{2}}{b}\right)^{2}\right)^{1.5000}} \\
& q_{12}=0.0185 \mathrm{k}_{\mathrm{c}}\left(32 \alpha^{3} b^{2} \mathrm{k}_{\mathrm{a}}{ }^{2} \mathrm{k}_{\mathrm{c}}{ }^{2} \omega_{\max }{ }^{2}+108 \alpha^{3} \mathrm{k}_{\mathrm{c}}{ }^{6}+16 \alpha^{2} b^{3} \mathrm{k}_{\mathrm{a}}{ }^{4}\right. \\
& \omega_{\max }^{2}+83.1384 \alpha^{2} b^{2} \mathrm{k}_{\mathrm{a}}{ }^{3} \mathrm{k}_{\mathrm{c}}{ }^{2} \omega_{\max }+324 \alpha^{2} b \\
& \mathrm{k}_{\mathrm{a}}{ }^{2} \mathrm{k}_{\mathrm{c}}{ }^{4}+41.5692 \alpha b^{3} \mathrm{k}_{\mathrm{a}}{ }^{5} \omega_{\max }+378 \alpha b^{2} \mathrm{k}_{\mathrm{a}}{ }^{4} \mathrm{k}_{\mathrm{c}}{ }^{2}+135 b^{3} \mathrm{k}_{\mathrm{a}}{ }^{6} \text { ) } \\
& e_{13}=\frac{q_{13}}{\alpha^{2} b^{3}\left(\left(0.3849 \mathrm{k}_{\mathrm{a}} \omega_{\max }+\frac{0.5000 \mathrm{k}_{\mathrm{a}}{ }^{2}}{\alpha}\right)^{2}+\left(\frac{\mathrm{k}_{\mathrm{a}}{ }^{2}}{\alpha}+\frac{\mathrm{k}_{\mathrm{c}}{ }^{2}}{b}\right)^{2}\right)^{1.5000}}
\end{aligned}
$$




$$
\begin{aligned}
& q_{13}=0.0185 \mathrm{k}_{\mathrm{a}} \mathrm{k}_{\mathrm{c}}{ }^{2}\left(8 \alpha^{2} \mathrm{k}_{\mathrm{c}}{ }^{2} \omega_{\max }{ }^{2}-8 b \alpha \mathrm{k}_{\mathrm{a}}{ }^{2} \omega_{\max }{ }^{2}+31.1769 \alpha \mathrm{k}_{\mathrm{a}} \mathrm{k}_{\mathrm{c}}{ }^{2} \omega_{\max }-10.3923 b \mathrm{k}_{\mathrm{a}}{ }^{3} \omega_{\max }+27 \mathrm{k}_{\mathrm{a}}{ }^{2} \mathrm{k}_{\mathrm{c}}{ }^{2}\right) \quad(\mathrm{C} .16) \\
& e_{21}=e_{12} \\
& e_{22}=\frac{q_{22}}{\alpha^{3} b^{4}\left(\left(0.3849 \mathrm{k}_{\mathrm{a}} \omega_{\max }+\frac{0.5000 \mathrm{k}_{\mathrm{a}}{ }^{2}}{\alpha}\right)^{2}+\left(\frac{\mathrm{k}_{\mathrm{a}}{ }^{2}}{\alpha}+\frac{\mathrm{k}_{\mathrm{c}}{ }^{2}}{b}\right)^{2}\right)^{1.5000}} \\
& q_{22}=0.0185\left(48 \alpha^{3} b^{2} \mathrm{k}_{\mathrm{a}}{ }^{2} \mathrm{k}_{\mathrm{c}}{ }^{2} \omega_{\max }{ }^{2}+108 \alpha^{3} \mathrm{k}_{\mathrm{c}}{ }^{6}+16 \alpha^{2} b^{3} \mathrm{k}_{\mathrm{a}}{ }^{4} \omega_{\max }{ }^{2}+124.7077 \alpha^{2} b^{2}\right. \\
& \mathrm{k}_{\mathrm{a}}{ }^{3} \mathrm{k}_{\mathrm{c}}{ }^{2} \omega_{\max }+324 \alpha^{2} b \mathrm{k}_{\mathrm{a}}{ }^{2} \mathrm{k}_{\mathrm{c}}{ }^{4}+41.5692 \alpha b^{3} \mathrm{k}_{\mathrm{a}}{ }^{5} \omega_{\max }+405 \alpha b^{2} \mathrm{k}_{\mathrm{a}}{ }^{4} \mathrm{k}_{\mathrm{c}}{ }^{2}+135 b^{3} \mathrm{k}_{\mathrm{a}}{ }^{6} \text { ) (C.19) } \\
& e_{23}=-\frac{0.0370 \mathrm{k}_{\mathrm{a}} \mathrm{k}_{\mathrm{c}}\left(8 \alpha^{2} \mathrm{k}_{\mathrm{c}}{ }^{2} \omega_{\max }{ }^{2}-8 b \alpha \mathrm{k}_{\mathrm{a}}{ }^{2} \omega_{\max }{ }^{2}+31.1769 \alpha \mathrm{k}_{\mathrm{a}} \mathrm{k}_{\mathrm{c}}{ }^{2} \omega_{\max }-10.3923 b \mathrm{k}_{\mathrm{a}}{ }^{3} \omega_{\max }+27 \mathrm{k}_{\mathrm{a}}{ }^{2} \mathrm{k}_{\mathrm{c}}{ }^{2}\right)}{\alpha^{2} b^{2}\left(\left(0.3849 \mathrm{k}_{\mathrm{a}} \omega_{\max }+\frac{0.5000 \mathrm{k}_{\mathrm{a}}{ }^{2}}{\alpha}\right)^{2}+\left(\frac{\mathrm{k}_{\mathrm{a}}{ }^{2}}{\alpha}+\frac{\mathrm{k}_{\mathrm{c}}{ }^{2}}{b}\right)^{2}\right)^{1.5000}} \\
& e_{31}=e_{13} \\
& e_{32}=e_{23}
\end{aligned}
$$




$$
\begin{gathered}
e_{33}=\frac{q_{33}}{\alpha^{4} b^{3}\left(\left(0.3849 \mathrm{k}_{\mathrm{a}} \omega_{\max }+\frac{0.5000 \mathrm{k}_{\mathrm{a}}{ }^{2}}{\alpha}\right)^{2}+\left(\frac{\mathrm{k}_{\mathrm{a}}{ }^{2}}{\alpha}+\frac{\mathrm{k}_{\mathrm{c}}{ }^{2}}{b}\right)^{2}\right)^{1.5000}} \\
q_{33}=5.1440 e-04\left(288 \alpha^{4} b \mathrm{k}_{\mathrm{c}}{ }^{4} \omega_{\max }{ }^{2}+110.8513 \alpha^{3} b^{3} \mathrm{k}_{\mathrm{a}}{ }^{3} \omega_{\max }{ }^{3}+2.2447 e+03 \alpha^{3} b \mathrm{k}_{\mathrm{a}} \mathrm{k}_{\mathrm{c}}{ }^{4} \omega_{\max }+3888\right. \\
\alpha^{3} \mathrm{k}_{\mathrm{c}}{ }^{6}+1296 \alpha^{2} b^{3} \mathrm{k}_{\mathrm{a}}{ }^{4} \omega_{\max }{ }^{2}+1.4965 e+03 \alpha^{2} b^{2} \mathrm{k}_{\mathrm{a}}{ }^{3} \mathrm{k}_{\mathrm{c}}{ }^{2} \omega_{\max }+14580 \alpha^{2} b \mathrm{k}_{\mathrm{a}}{ }^{2} \\
\left.\mathrm{k}_{\mathrm{c}}{ }^{4}+2.8059 e+03 \alpha b^{3} \mathrm{k}_{\mathrm{a}}{ }^{5} \omega_{\max }+14580 \alpha b^{2} \mathrm{k}_{\mathrm{a}}{ }^{4} \mathrm{k}_{\mathrm{c}}{ }^{2}+6075 b^{3} \mathrm{k}_{\mathrm{a}}{ }^{6}\right)
\end{gathered}
$$

and for Phase 2 as

$$
\begin{gathered}
\frac{d}{d \boldsymbol{p}} u_{P 2}\left(v_{\max }\right)=2 \omega_{\max }+\frac{2 \mathrm{f} \pi v_{\max }\left(\frac{1.1382}{\theta_{d}}+0.7246\right)}{\alpha^{\prime}} \\
\frac{d^{2}}{d \boldsymbol{p}^{2}} u_{P 2}\left(v_{\max }\right)=\frac{0.7246 \mathrm{f} \pi\left(2 \theta_{d}+3.1416\right)}{\alpha^{\prime} \theta_{d}}
\end{gathered}
$$

The performance cost Jacobians and Hessians are also solved using MATLAB symbolic. For Phase 1, these matrices are

$$
\begin{gathered}
\frac{d J_{P 1}}{d \boldsymbol{p}_{P 1}}=\left[\begin{array}{c}
\frac{2 W_{t} b}{\mathrm{k}_{\mathrm{c}}}-\frac{W_{j} \mathrm{k}_{\mathrm{c}}{ }^{2}}{b^{2}} \\
W_{f}\left(\frac{\hat{\boldsymbol{r}}_{0}^{T}\left(\dot{\boldsymbol{r}}_{0}+k_{c} \hat{\boldsymbol{r}}_{0}\right)}{\left\|\dot{\boldsymbol{r}}_{0}+k_{c} \hat{\boldsymbol{r}}_{0}\right\|}+1\right)-W_{t}\left(\frac{1}{\mathrm{k}_{\mathrm{c}}{ }^{2}}+\frac{b^{2}}{\mathrm{k}_{\mathrm{c}}{ }^{2}}\right)+\frac{2 W_{j} \mathrm{k}_{\mathrm{c}}}{b} \\
-W_{f}\left(\frac{6.5000 \alpha^{2}\left\|\boldsymbol{\omega}_{\mathrm{BI}}^{\times} \boldsymbol{\omega}_{\mathrm{BI}}^{\times}+\dot{\boldsymbol{\omega}}_{\mathrm{BI}}^{\times}\right\|_{\max }}{\mathrm{k}_{\mathrm{a}}{ }^{2}}-1.5708\right)-\frac{6.5000 W_{t} \alpha}{\mathrm{k}_{\mathrm{a}}{ }^{2}}
\end{array}\right] \\
\frac{d^{2} J}{d \boldsymbol{p}_{P 1}^{2}}=\left[\begin{array}{lll}
m_{11} & m_{12} & m_{13} \\
m_{21} & m_{22} & m_{23} \\
m_{31} & m_{32} & m_{33}
\end{array}\right]
\end{gathered}
$$


where

$$
\begin{gathered}
m_{11}=\frac{2 W_{t}}{\mathrm{k}_{\mathrm{c}}}+\frac{2 W_{j} \mathrm{k}_{\mathrm{c}}{ }^{2}}{b^{3}} \\
m_{12}=-\frac{2 W_{j} \mathrm{k}_{\mathrm{c}}}{b^{2}}-\frac{2 W_{t} b}{\mathrm{k}_{\mathrm{c}}{ }^{2}} \\
m_{13}=0 \\
m_{21}=-\frac{2 W_{j} \mathrm{k}_{\mathrm{c}}}{b^{2}}-\frac{2 W_{t} b}{\mathrm{k}_{\mathrm{c}}{ }^{2}} \\
m_{22}=\frac{2 W_{j}}{b}+\frac{W_{f}\left(l_{22}\right)}{\left\|\dot{\boldsymbol{r}}_{0}+k_{c} \hat{\boldsymbol{r}}_{0}\right\|^{3}}+\frac{2 W_{t}\left(b^{2}+1\right)}{\mathrm{k}_{\mathrm{c}}{ }^{3}}
\end{gathered}
$$

$l_{22}=\hat{\mathrm{r}}_{0, \mathrm{x}}^{2} \dot{\mathrm{r}}_{0, \mathrm{y}}^{2}+\hat{\mathrm{r}}_{0, \mathrm{x}}^{2} \dot{\mathrm{r}}_{0, \mathrm{z}}^{2}-2 \hat{\mathrm{r}}_{0, \mathrm{x}} \hat{\mathrm{r}}_{0, \mathrm{y}} \dot{\mathrm{r}}_{0, \mathrm{x}} \dot{\mathrm{r}}_{0, \mathrm{y}}-2 \hat{\mathrm{r}}_{0, \mathrm{x}} \hat{\mathrm{r}}_{0, \mathrm{z}} \dot{\mathrm{r}}_{0, \mathrm{x}} \dot{\mathrm{r}}_{0, \mathrm{z}}+$

$$
\hat{\mathrm{r}}_{0, \mathrm{y}}^{2} \dot{\mathrm{r}}_{0, \mathrm{x}}^{2}+\hat{\mathrm{r}}_{0, \mathrm{y}}^{2} \dot{\mathrm{r}}_{0, \mathrm{z}}^{2}-2 \hat{\mathrm{r}}_{0, \mathrm{y}} \hat{\mathrm{r}}_{0, \mathrm{z}} \dot{\mathrm{r}}_{0, \mathrm{y}} \dot{\mathrm{r}}_{0, \mathrm{z}}+\hat{\mathrm{r}}_{0, \mathrm{z}}^{2} \dot{\mathrm{r}}_{0, \mathrm{x}}^{2}+\hat{\mathrm{r}}_{0, \mathrm{z}}^{2} \dot{\mathrm{r}}_{0, \mathrm{y}}^{2} \quad \text { (C.34) }
$$

$$
\begin{gathered}
m_{23}=0 \\
m_{31}=0 \\
m_{32}=0 \\
m_{33}=\frac{13 \alpha\left(W_{t}+W_{f} \alpha\left\|\boldsymbol{\omega}_{\mathrm{BI}}^{\times} \boldsymbol{\omega}_{\mathrm{BI}}^{\times}+\dot{\boldsymbol{\omega}}_{\mathrm{BI}}^{\times}\right\|_{\max }\right)}{\mathrm{k}_{\mathrm{a}}{ }^{3}}
\end{gathered}
$$


For Phase 2, these equations are

$$
\frac{d J_{P 2}}{d \boldsymbol{p}_{P 2}}=-\frac{0.0100\left(831 W_{t} \alpha^{\prime} \mathrm{f} \pi-200 W_{f}(\mathrm{f} \pi)^{2} \mathrm{v}_{\max }{ }^{2}+1305 W_{f} \alpha^{\prime 2}\left\|\boldsymbol{\omega}_{\mathrm{BI}}^{\times} \boldsymbol{\omega}_{\mathrm{BI}}^{\times}+\dot{\boldsymbol{\omega}}_{\mathrm{BI}}^{\times}\right\|_{\max }+831 W_{f} \alpha^{\prime} \mathrm{f} \pi\right)}{(\mathrm{f} \pi)^{2} \mathrm{v}_{\max }{ }^{2}}
$$

$\frac{d^{2} J_{P 2}}{d \boldsymbol{p}_{P 2}}=\frac{4 W_{f}}{\mathrm{v}_{\max }}+\frac{0.0200\left(831 W_{t} \alpha^{\prime} \mathrm{f} \pi-200 W_{f}(\mathrm{f} \pi)^{2} \mathrm{v}_{\max }{ }^{2}+1305 W_{f} \alpha^{\prime 2}\left\|\boldsymbol{\omega}_{\mathrm{BI}}^{\times} \boldsymbol{\omega}_{\mathrm{BI}}^{\times}+\dot{\boldsymbol{\omega}}_{\mathrm{BI}}^{\times}\right\|_{\max }+831 W_{f} \alpha^{\prime}\left\|\left(\boldsymbol{\omega}_{B I}^{\times} \boldsymbol{\omega}_{B I}^{\times}+\dot{\boldsymbol{\omega}}_{B I}^{\times}\right) \boldsymbol{d}\right\| \|_{\max } \mathrm{f} \pi\right)}{(f \pi)^{2} \mathrm{v}_{\max }{ }^{3}}$ 


\section{References}

[1] Kessler, D. J. and Cour-Palais, B. G., "Collision frequency of artificial satellites: The creation of a debris belt," Journal of Geophysical Research: Space Physics, Vol. 83, No. A6, 1978, pp. 2637-2646.

[2] Eckersley, S., Saunders, C., Gooding, D., Sweeting, M., Whiting, C., Ferris, M., Friend, J., Forward, L., Aglietti, G., Nanjangud, A., et al., "In-Orbit Assembly of Large Spacecraft Using Small Spacecraft and Innovative Technologies," Proceedings of the 69th International Astronautical Congress (IAC), International Astronautical Federation (IAF), 2018.

[3] Hamill, D., Jefferies, S. A., Moses, R. W., Stillwagen, F., Mullins, C., and Gresham, E., "Space Science and Technology Partnership Forum: Analysis for a Joint Demonstration of High Priority, In-Space Assembly Technology," 2018 AIAA SPACE and Astronautics Forum and Exposition, 2018, p. 5307.

[4] Weiss, A., Baldwin, M., Erwin, R. S., and Kolmanovsky, I., "Model predictive control for spacecraft rendezvous and docking: Strategies for handling constraints and case studies," IEEE Transactions on Control Systems Technology, Vol. 23, No. 4, 2015, pp. 1638-1647.

[5] Di Cairano, S., Park, H., and Kolmanovsky, I., "Model predictive control approach for guidance of spacecraft rendezvous and proximity maneuvering," International Journal of Robust and Nonlinear Control, Vol. 22, No. 12, 2012, pp. $1398-1427$.

[6] Li, Q., Yuan, J., Zhang, B., and Gao, C., "Model predictive control for autonomous rendezvous and docking with a tumbling target," Aerospace Science and Technology, Vol. 69, 2017, pp. 700-711.

[7] Park, H., Zagaris, C., Virgili Llop, J., Zappulla, R., Kolmanovsky, I., and Romano, M., "Analysis and experimentation of model predictive control for spacecraft rendezvous and proximity operations with multiple obstacle avoidance," AIAA/AAS Astrodynamics Specialist Conference, 2016, p. 5273.

[8] Zhu, S., Sun, R., Wang, J., Wang, J., and Shao, X., "Robust model predictive control for multi-step short range spacecraft rendezvous," Advances in Space Research, Vol. 62, No. 1, 2018, pp. 111-126.

[9] Ogata, K., System Dynamics, Pearson Education Inc., 2004, pp. 492-493.

[10] De Ruiter, A. H., Damaren, C., and Forbes, J. R., Spacecraft Dynamics and Control: an Introduction, John Wiley \& Sons, 2012, pp. 235-245. 
[11] Ventura, J., Ciarcia, M., Romano, M., and Walter, U., "An inverse dynamicsbased trajectory planner for autonomous docking to a tumbling target," AIAA Guidance, Navigation, and Control Conference, 2016, p. 0876.

[12] Lopez, I. and McInnes, C. R., "Autonomous rendezvous using artificial potential function guidance," Journal of Guidance, Control, and Dynamics, Vol. 18, No. 2, 1995, pp. 237-241.

[13] Hassan, K., Nonlinear Systems, chap. 4, Prentice-Hall, Inc., New Jersey, 1996.

[14] Lawrence, D., "Lyapunov vector fields for UAV flock coordination," 2nd AIAA "Unmanned Unlimited" Conf. and Workshop $\&$ Exhibit, 2003, p. 6575.

[15] Frew, E. W., Lawrence, D. A., and Morris, S., "Coordinated standoff tracking of moving targets using Lyapunov guidance vector fields," Journal of Guidance, Control, and Dynamics, Vol. 31, No. 2, 2008, pp. 290-306.

[16] Pothen, A. A. and Ratnoo, A., "Curvature-constrained Lyapunov vector field for standoff target tracking," Journal of Guidance, Control, and Dynamics, Vol. 40, No. 10, 2017, pp. 2729-2736.

[17] Lawrence, D. A., Frew, E. W., and Pisano, W. J., "Lyapunov vector fields for autonomous unmanned aircraft flight control," Journal of Guidance, Control, and Dynamics, Vol. 31, No. 5, 2008, pp. 1220-1229.

[18] Scorsoglio, A. and Furfaro, R., "ELM-based Actor-Critic Approach to Lyapunov Vector Fields Relative Motion Guidance in Near-Rectilinear Orbit," 2019 AAS/AIAA Astrodynamics Specialists Conference, 2019, pp. 1-20.

[19] Hough, J. G. and Ulrich, S., "Lyapunov Vector Fields for Thrust-Limited Spacecraft Docking with an Elliptically-Orbiting Uncooperative Tumbling Target," AIAA Guidance, Navigation, and Control Conference, 2020, p. 2078.

[20] Sun, S., Wang, H., Liu, J., and He, Y., "Fast Lyapunov vector field guidance for standoff target tracking based on offline search," IEEE Access, Vol. 7, 2019, pp. 124797-124808.

[21] Che, F., Niu, Y., Li, J., and Wu, L., "Cooperative Standoff Tracking of Moving Targets Using Modified Lyapunov Vector Field Guidance," Applied Sciences, Vol. 10, No. 11, 2020, pp. 3709.

[22] Chen, H., Chang, K., and Agate, C. S., "UAV path planning with tangent-plusLyapunov vector field guidance and obstacle avoidance," IEEE Transactions on Aerospace and Electronic Systems, Vol. 49, No. 2, 2013, pp. 840-856.

[23] Wilhelm, J. P. and Clem, G., "Vector field UAV guidance for path following and obstacle avoidance with minimal deviation," Journal of Guidance, Control, and Dynamics, Vol. 42, No. 8, 2019, pp. 1848-1856. 
[24] De Ruiter, A. H., Damaren, C., and Forbes, J. R., Spacecraft Dynamics and Control: an Introduction, chap. 26, John Wiley \& Sons, 2012, pp. 506-511.

[25] Larson, W. and Wertz, J., Space Mission Analysis and Design, chap. 11, Microcosm, Inc., 1992.

[26] Khalil, H. K., Lyapunov's Stability Theory, Springer London, London, 2013, pp. $1-6$.

[27] Caveny, J., "On integral Lipschitz conditions and integral bounded variation," Journal of the London Mathematical Society, Vol. 2, No. 2, 1970, pp. 346-352.

[28] Boyd, S., Boyd, S. P., and Vandenberghe, L., Convex optimization, chap. 9, Cambridge university press, 2004, pp. 464-466.

[29] De Ruiter, A. H., Damaren, C., and Forbes, J. R., Spacecraft Dynamics and Control: an Introduction, chap. 2, John Wiley \& Sons, 2012, pp. 58-60.

[30] Schaub, H. and Junkins, J. L., Analytical Mechanics of Space Systems, American Institute of Aeronautics and Astronautics, 2005, pp. 676-678.

[31] Paluszek, M. and Thomas, S., "Generalized 3D Spacecraft Proximity Path Planning Using A," Infotech@ Aerospace, Arlington, Virginia, 2005, p. 7043.

[32] Setterfield, T. P., Miller, D. W., Saenz-Otero, A., Frazzoli, E., and Leonard, J. J., "Inertial Properties Estimation of a Passive On-orbit Object Using Polhode Analysis," Journal of Guidance, Control, and Dynamics, Vol. 41, No. 10, 2018, pp. 2214-2231.

[33] Tong, D., "Spacecraft momentum dumping using gravity gradient," Journal of spacecraft and rockets, Vol. 35, No. 5, 1998, pp. 714-717.

[34] Boyd, S., Boyd, S. P., and Vandenberghe, L., Convex optimization, chap. 3, Cambridge university press, 2004.

[35] Boyd, S., Boyd, S. P., and Vandenberghe, L., Convex optimization, chap. 11, Cambridge university press, 2004, pp. 562-565.

[36] Efimov, S., Pritykin, D., and Sidorenko, V., "Long-term attitude dynamics of space debris in Sun-synchronous orbits: Cassini cycles and chaotic stabilization," Celestial Mechanics and Dynamical Astronomy, Vol. 130, No. 10, 2018, pp. 1-25. 